\title{
New concepts in ultrasound image processing and their potential for improving data presentation
}

Citation for published version (APA):

Collaris, R. J. (1996). New concepts in ultrasound image processing and their potential for improving data presentation. [Doctoral Thesis, Maastricht University]. Rijksuniversiteit Limburg. https://doi.org/10.26481/dis.19960613rc

Document status and date:

Published: 01/01/1996

DOI:

10.26481/dis.19960613rc

Document Version:

Publisher's PDF, also known as Version of record

\section{Please check the document version of this publication:}

- A submitted manuscript is the version of the article upon submission and before peer-review. There can be important differences between the submitted version and the official published version of record.

People interested in the research are advised to contact the author for the final version of the publication, or visit the DOI to the publisher's website.

- The final author version and the galley proof are versions of the publication after peer review.

- The final published version features the final layout of the paper including the volume, issue and page numbers.

Link to publication

\footnotetext{
General rights rights.

- You may freely distribute the URL identifying the publication in the public portal. please follow below link for the End User Agreement:

www.umlib.nl/taverne-license

Take down policy

If you believe that this document breaches copyright please contact us at:

repository@maastrichtuniversity.nl

providing details and we will investigate your claim.
}

Copyright and moral rights for the publications made accessible in the public portal are retained by the authors and/or other copyright owners and it is a condition of accessing publications that users recognise and abide by the legal requirements associated with these

- Users may download and print one copy of any publication from the public portal for the purpose of private study or research.

- You may not further distribute the material or use it for any profit-making activity or commercial gain

If the publication is distributed under the terms of Article $25 \mathrm{fa}$ of the Dutch Copyright Act, indicated by the "Taverne" license above, 
New Concepts in Ultrasound Image Processing and their potential for improving data presentation 



\section{New Concepts in Ultrasound Image Processing and their potential for improving data presentation}

\section{Proefschrift}

ter verkrijging van de graad van doctor aan de Rijksuniversiteit Limburg te Maastricht, op gezag van de Rector Magnificus, Prof. mr. M.J. Cohen, volgens het besluit van het College van Dekanen, in het openbaar te verdedigen op donderdag 13 juni 1996 om 14.00 uur

$$
\text { door }
$$

\section{Roland Jozef Collaris}

geboren te Heerlen op 9 augustus 1967 
Promotor:

Prof. dr. ir. A.P.G. Hoeks

Beoordelingscommissie: Prof. dr. ir. A. Hasman (voorzitter)

Prof. dr. J.M.A. van Engelshoven

Prof. dr. R.S. Reneman

Dr. ir. J.M. Thijssen (Katholieke Universiteit Nijmegen)

Prof. dr. ir. drs. O.J. Vrieze

Het verschijnen van dit proefschrift werd mede mogelijk gemaakt door financiële steun van:

- de Nederlandse Hartstichting

- Pie Medical Benelux BV 
Aan mijn moeder en aan Jacqueline 

$\begin{array}{lll}1.1 & \text { Aim of the study } & 14\end{array}$

$\begin{array}{ll}1.2 & \text { Outline of the thesis } \\ \end{array}$

2 Medical Ultrasound $\quad 17$

2.1 Basic properties 20

2.2 Emission and reception 23

$\begin{array}{lll}2.3 & \text { B-mode echo imaging } & 28\end{array}$

2.3.1 Amplitude detection 30

2.3.2 Scan conversion $\quad 33$

2.3.3 Gray level transform and display $\quad 35$

2.3.4 Postprocessing 36

$\begin{array}{ll}2.4 & \text { Blood velocity measurement }\end{array}$

2.4.1 Doppler processing 41

2.4.2 Rf correlation interpolation $\quad 55$

$\begin{array}{lll}2.5 & \text { Color velocity imaging } & 60\end{array}$

2.5.1 Postprocessing of velocity and parameter distributions 62

2.5.2 Display of color velocity images 64

3 Information Limitations and Human Vision $\quad 71$

$\begin{array}{lll}3.1 & \text { B-mode echo imaging } & 73\end{array}$

$\begin{array}{lll}3.2 & \text { Real-time display of color velocity images } & 78\end{array}$

4 Ultrasonic B-Mode Image Enhancement

based on Level-Dependent Spread Functions $\quad 81$

$\begin{array}{lll}4.1 \text { Abstract } & 84\end{array}$

4.2 Introduction $\quad 84$

4.3 Level-dependent spatial summation $\quad 86$

$\begin{array}{lll}\text { 4.3.1 Method } & 86\end{array}$

$\begin{array}{lll}\text { 4.3.2 Application to ultrasonic imaging } & 88\end{array}$

4.3.3 Properties of the block-shaped amplitude-dependent spread function $\quad 90$

$\begin{array}{lll}4.4 & \text { Image enhancement } & 92\end{array}$

$\begin{array}{lll}\text { 4.4.1 Speckle reduction } & 92\end{array}$

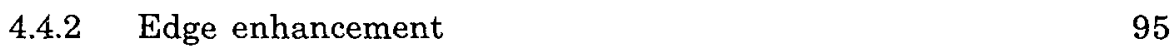

$\begin{array}{ll}\text { 4.4.3 Contrast enhancement } & 100\end{array}$

$\begin{array}{ll}4.5 & \text { Level of improvement } \\ & 101\end{array}$

$\begin{array}{lll}4.6 & \text { Discussion } & 104\end{array}$

$\begin{array}{lll}4.7 & \text { Conclusion } & 106\end{array}$ 
5 Improved Lesion Detection

by Level-Dependent Spatial Summation

$\begin{array}{lll}5.1 \text { Abstract } & 111\end{array}$

$\begin{array}{ll}5.2 & \text { Introduction } \\ 5.3 & \text { Method }\end{array}$

$\begin{array}{lll}5.3 & \text { Method } & 113\end{array}$

5.4 Theoretical analysis 114

5.4.1 Response to a stationary amplitude pattern 114

$\begin{array}{ll}\text { 5.4.2 Transient response } & 119\end{array}$

$\begin{array}{ll}5.5 & \text { Experimental verification } \\ 5.6 & 121\end{array}$

$\begin{array}{ll}5.6 & \text { Lesion detection } \\ 5.7 & 126\end{array}$

5.7 Discussion $\quad 135$

$\begin{array}{lll}5.8 \text { Conclusion } & 137\end{array}$

$\begin{array}{ll}\text { Appendix A } & 139\end{array}$

$\begin{array}{lr}\text { Appendix B } & 140\end{array}$

6 Postprocessing of Velocity Distributions

in Real-Time Ultrasonic Color Velocity Imaging 143

$\begin{array}{lll}6.1 \text { Abstract } & 145\end{array}$

6.2 Introduction $\quad 145$

$\begin{array}{ll}6.3 & \text { Assumptions } \\ & 147\end{array}$

$\begin{array}{ll}\text { 6.4 Description of the algorithm } & 148\end{array}$

$\begin{array}{lll}\text { 6.4.1 Modification } 1 & 149\end{array}$

6.4.2 Modification 2 155

6.5 Application to artificial and real velocity signals 156

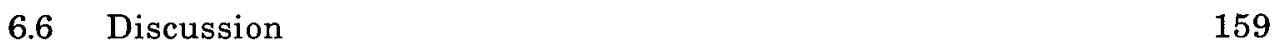

$\begin{array}{lll}6.7 & \text { Conclusion } & 161\end{array}$

$\begin{array}{ll}\text { Appendix } & 162\end{array}$

7 Application of Level-Dependent Spatial Summation to Clinical Data

$\begin{array}{lll}7.1 & \text { Introduction } & 165\end{array}$

7.1.1 Level-Dependent spatial Summation $\quad 165$

7.1.2 Aim of the experiments 168

$\begin{array}{ll}7.2 & \text { Experimental setup } \\ & 168\end{array}$

$\begin{array}{lll}7.2 .1 & \text { Equipment } & 168\end{array}$

$\begin{array}{lll}7.2 .2 & \text { Data collection } & 168\end{array}$

7.2.3 Signal processing 169

7.2.4 Quantification of the level of improvement 169

$\begin{array}{lll}\text { 7.3 Processing B-mode data of the normal gallbladder } & 170\end{array}$

$\begin{array}{ll}7.4 & \text { Processing B-mode data of a breast tumor } \\ \end{array}$ 
7.5 Processing B-mode data of the normal liver

7.6 Concluding remarks

8 Discussion and Conclusions 183

$\begin{array}{ll}8.1 \text { Discussion } & 185\end{array}$

$\begin{array}{lll}8.2 & \text { Conclusions } & 187\end{array}$

$\begin{array}{ll}\text { Summary } & 189\end{array}$

$\begin{array}{ll}\text { Samenvatting } & 193\end{array}$

$\begin{array}{ll}\text { Publications } & 197\end{array}$

$\begin{array}{ll}\text { Nawoord } & 198\end{array}$

$\begin{array}{ll}\text { Curriculum Vitae } & 198\end{array}$ 

1

\section{Introduction}


Ultrasound enjoys a widespread clinical use as a valuable source of diagnostic information. The success of medical ultrasonic imaging can be attributed to a number of important qualities that other imaging modalities lack or, at least, exhibit to a lesser extent. Ultrasound is supposed to be a rather safe form of radiation under the low doses commonly applied during clinical routine investigation. Ultrasound equipment is relatively cheap in acquisition, use and maintenance. No special provisions or preparations are required, making on-bed application possible. A wide variety of organs, including flowing blood, is open to ultrasound investigation. Target interrogation is rapid, enabling dynamic processes to be visualized. Diagnostic information becomes available as soon as measurements have started, and real-time imaging is supported. The virtual absence of a time lag between data collection and data presentation enables the human observer to optimize the field of view interactively.

Opposed to these strengths, ultrasonic imaging suffers certain shortcomings that are related to the physical characteristics of ultrasound. Part of the less favorable properties are especially associated with two very common imaging modes: brightness mode or $B$-mode echo imaging and color velocity imaging. These limitations will be of concern in the present study.

In B-mode echo imaging, a two-dimensional gray level image of a planar section of a target is constructed on the basis of its echo response to a series of ultrasound pulses applied. Although the image is essentially a reflection of the distribution of acoustic inhomogeneities present in the plane of observation, anatomical organ information can be extracted by visual inspection of the image, provided that adjacent structures produce sufficient contrast. However, B-mode echo images have a typical mottled appearance. The (pseudo) random pattern of light and dark grains, called speckle, is a result of interference of ultrasound echoes. Speckle noise is generally viewed as a major source of image degradation. Having a coefficient of variation of about 0.5 , speckle noise hampers the perception of subtle differences in local mean gray level, which may provide diagnostic information about, e.g., the presence of a tumor in an organ. Common signal postprocessing methods achieve speckle reduction while sacrificing part of the image resolution in space or time.

The other imaging mode that is part of the present study is color velocity imaging. In this mode of operation, ultrasound based blood velocity measurement is added to B-mode echo imaging to produce, in real-time, a gray-level anatomical image in which the lumens of vessels and other blood containing regions are filled with instantaneous, spatial blood velocity profiles, encoded in color. Rapid refreshment of color velocity images on a monitor screen yields a cinematographic effect 
that allows local blood flow dynamics to be assessed. A major annoyance of color velocity imaging is, that the spatial blood velocity profiles of consecutive images lack consistency. The frayed boundaries of the profiles, where color encoded velocity pixels touch gray level anatomical pixels, tend to change randomly from one image to the next. This is a result of a relatively poor temporal resolution (typically 10 independent images per second) in combination with a short observation time of local blood flow (of the order of $1 \mathrm{~ms}$ ). As a consequence, the images exhibit in realtime observation a pronounced unrest that is likely to hamper the extraction of relevant diagnostic information and, moreover, that causes blood containing regions to appear ill-defined in the image. To overcome this problem, commercial color velocity systems are often equipped with some kind of persistence filter that provides temporal smoothing of the blood velocity distributions. As a side effect, however, a discrepancy is invoked, especially under dynamic flow circumstances, between the actual blood flow behavior and the velocity information displayed.

\subsection{Aim of the study}

- The conception, analysis and evaluation of an ultrasound B-mode signal postprocessing technique that (1) reduces the adverse effect of speckle while preserving resolution, that (2) increases the contrast between different structures in the image, and that (3) enhances the edges of structures in the image. The method should lead to an improved presentation of the B-mode data recorded, and facilitate the extraction of the structural information by the human observer. The operations involved in the processing of an image should be simple and restricted in number, so that real-time implementation is feasible.

- The conception, analysis and evaluation of a signal postprocessing method in color velocity imaging, that leads to an improved consistency between velocity distributions of consecutive images, and an improved definition of the regions containing flowing blood. The true blood velocity information measured should not be affected and real-time implementation should be supported.

\subsection{Outline of the thesis}

Chapters 2 and 3 provide a frame work for the new approaches to ultrasound image processing that are presented in Chapters 4 to 6 . Chapters 2 and 3 enable a concrete and detailed definition of the issues of the present study, and they put the 
algorithms to be proposed in perspective.

Chapter 2 deals with the major aspects of medical ultrasound, ultrasound Bmode imaging, ultrasound based blood velocity measurement, and color velocity imaging. Reflections are made with respect to various technical facets that are of importance to the ideas of Chapters 4 to 6 . Supplementary references to existing literature are given. In addition, three computer simulation experiments are described. In one experiment, a comparison is made between two fast algorithms for calculating instantaneous ultrasound echo amplitude (as is an essential part of Bmode echo imaging). In each of the other two experiments, one particular blood velocity estimation technique is evaluated as regards capacity to discriminate between true and false blood velocity estimates (as is an essential part of color velocity imaging). Finally, velocity discrimination is illustrated on the basis of actual color velocity data recorded from a carotid artery.

Chapter 3 investigates the major limitations of ultrasonic imaging with respect to the detail to which targets can be investigated and the extent to which the information gathered can be absorbed by the human observer. An overview is given of common existing techniques that try to overcome (part of) the limitations. This way, a context is created for the new techniques of Chapters 4 to 6 .

In Chapter 4, a new ultrasound B-mode signal postprocessing technique is proposed, in which speckle reduction, edge enhancement and enhancement of contrast between different structures in the image, are integrated. Basically, in a process that will be called "Level-Dependent spatial Summation", two spatial signal distributions are derived from a B-mode image: one (the " $M$ " distribution) includes information about high spatial frequencies in the B-mode image, the other (the " $N$ " distribution) about low frequencies. The $M$ and $N$ distributions are employed to enhance the original image. Theoretical reflections are made and results are presented of the processing of computer simulated images and images recorded from a tissue mimicking phantom.

In Chapter 5, the $N$ distribution introduced in Chapter 4 is regarded as an independent process. The algorithm is generalized and the properties of the $N$ distribution are investigated more profoundly. A theoretical analysis is presented as well as results of computer simulation experiments, showing that the $N$ distribution, displayed as a gray level image, leads to an improved detectability of focal lesions in Bmode images, such as tumors.

Chapter 6 deals with color velocity imaging. The lack of consistency in a se- 
quence of consecutive blood velocity profiles is tackled. A velocity signal postprocessing method is introduced that attempts to recover the consistency, reduce the unrest perceived in real-time observation, and improve the definition in the image of the regions that contain flowing blood. Effort is directed to the preservation of all true velocity information measured. Results are presented of the application of the algorithm to signals recorded from a flow phantom.

In Chapter 7, three cases are worked out of the application of the methods of Chapters 4 and 5 to actual clinical B-mode data. The techniques developed are subsequently applied to a gallbladder image, an image of a breast tumor and a liver image.

Chapter 8 includes a global discussion of the study and the main conclusions. 


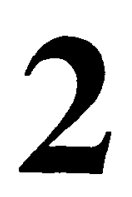

Medical Ultrasound 


\section{List of main symbols}

\begin{tabular}{|c|c|}
\hline$f$ & frequency (general) \\
\hline $\mathrm{i}, \mathrm{j}, \mathrm{n}$ & integer counters \\
\hline$t$ & time (general) \\
\hline$t_{y}, t_{v}$ & $\begin{array}{l}t_{y} \text { denotes time progress along an } \mathrm{rf} \text { signal, } t_{v} \text { denotes time progress } \\
\text { between different } \mathrm{rf} \text { signal recordings. }\end{array}$ \\
\hline$x, y$ & $\begin{array}{l}\text { space coordinates: } y \text { denotes axial position (along the beam axis) and } \\
x \text { denotes lateral position (perpendicular to the beam axis). }\end{array}$ \\
\hline
\end{tabular}

Symbols associated with (the generation of) a pulsed wave ultrasound field:

$\begin{array}{ll}\text { a } & \text { aperture radius of a piston transducer } \\ C_{u s} & \text { ultrasound pulse bandwidth } \\ f_{c} & \text { propagation speed of ultrasound } \\ F & \text { central ultrasound emission frequency } \\ Q & \text { focal distance } \\ \lambda & \text { ultrasound pulse quality factor } \\ \tau_{\mathrm{p}} & \text { ultrasound wavelength } \\ & \text { ultrasound pulse duration }\end{array}$

Symbols associated with the ultrasound echo signals received:
$A\left(t_{y}\right)$
echo amplitude
$f_{\mathrm{s}}, \tau_{\mathrm{s}}$
rf sampling frequency, rf sampling interval
PRF, $\tau_{\text {PRF }}$
pulse repetition frequency, pulse repetition interval
$r\left(t_{y}\right)$ radio frequency signal received
$\mathrm{SNR}_{\mathrm{rf}}$ rf signal-to-noise ratio

Symbols associated with blood velocity measurement:

$\begin{array}{ll}\text { BW } & \text { Doppler signal bandwidth } \\ C\left(\Delta t_{v}\right) & \text { complex Doppler signal autocorrelation coefficient } \\ f_{d} & \text { Doppler shift frequency } \\ G W & \text { gate width in number of sample points } \\ \mathrm{PL} & \text { package length in number of rf signals } \\ \mathrm{R}\left(\Delta \mathrm{t}_{\mathrm{y}}, \Delta \mathrm{t}_{\mathrm{v}}\right) & \text { real rf signal crosscorrelation coefficient } \\ \text { SNR }_{\text {Dop }} & \text { Doppler signal-to-noise ratio } \\ \text { SSNF }_{\mathrm{r}} & \text { rf signal-to-(signal+noise) fraction }\end{array}$




$\begin{array}{ll}\tau_{\text {trans }} & \text { transit time } \\ v & \text { scatterer velocity } \\ z\left(t_{v}\right) & \text { complex Doppler signal }\end{array}$

\subsection{Basic properties}

Medical ultrasound techniques employ the information carried by mechanical wavelets to gather knowledge about the condition of (part of) inner man. The phenomenon of pressure perturbations propagating through a medium is called sound. Because the frequencies used in medical applications extent far beyond the limit of human audible perception, the term ultrasound is used. Typical frequencies range from $2 \mathrm{MHz}$ in abdominal applications to $30 \mathrm{MHz}$ in intravascular applications (Bom et al., 1993). Ultrasound propagation is mainly longitudinal, whereby the wave velocity $c_{u s}\left[\mathrm{~m} \cdot \mathrm{s}^{-1}\right]$ is determined by the properties of the supporting medium (Wells, 1969):

$$
\mathrm{c}_{\mathrm{us}}=\sqrt{\mathrm{K} / \rho}
$$

where $K\left[\mathrm{~kg} \cdot \mathrm{m}^{-1} \cdot \mathrm{s}^{-2}\right]$ is the adiabatic bulk modulus of the material (Gere and Timoshenko, 1985) and $\rho\left[\mathrm{kg} \cdot \mathrm{m}^{-3}\right]$ is the density. It follows that the velocity increases with decreasing compressibility of the medium. In biological materials, $c_{u s}$ is largely independent of frequency. In addition, the variation of $c_{u s}$ over the different types of soft tissue is fairly small. The mean value approximates $1540 \mathrm{~m} / \mathrm{s}$ and most deviations are of the order of a few percents. The wavelength $\lambda[\mathrm{m}]$ is related to the frequency $f\left[\mathrm{~s}^{-1}\right]$ via:

$$
\lambda=\mathrm{c}_{\mathrm{us}} / \mathrm{f}
$$

Ultrasound is considered to be a relatively safe form of radiation as compared with other forms of radiation used in medical diagnosis. In this respect, the ultrasound intensity $I\left[\mathrm{~W} \cdot \mathrm{m}^{-2}\right]$ is an important parameter that is often related to possible, hazardous biological effects, like heating and cavitation (Neppiras, 1984; Ter Haar, 1986). $I$ is defined as the amount of wave energy transported (at velocity $c_{u s}$ ) through a unit area per unit time. For plane waves in lossless media, the intensity is related to the pressure amplitude $p\left[\mathrm{~N} \cdot \mathrm{m}^{-2}\right]$ according to:

$$
I=\frac{p^{2}}{2 \rho \cdot c_{u s}}
$$


In practice, ultrasound waves are mostly emitted in series of short bursts. In this respect, the spatial-peak-temporal-average (SPTA) intensity is often used to quantify ultrasound exposure (Wells, 1986; Wells et al., 1986). Levels of the SPTA intensity below $100 \mathrm{~mW} / \mathrm{cm}^{2}$ are commonly regarded as safe.

Various mechanisms influence the sound wave propagation through tissue. Fluctuations in local compressibility and density on the scale of the wavelength cause scattering: part of the impinging wave energy is radiated into directions other than the direction of main wave propagation. If the scale of the fluctuations is very small as compared with the wavelength, Rayleigh scattering occurs: the fluctuations act as point scatterers, uniformly directing a small part of the wave energy into all directions. Then, the scatter intensity is proportional to the fourth power of frequency. In general, scattering is the main mechanism utilized in tissue imaging and blood velocity measurement.

A second mechanism acting on the sound wave propagation is specular reflection and refraction, which occurs wherever the wavefront meets a global interface between structures having different acoustic properties. In those situations the geometric law applies that part of the wave is reflected at an angle equal to the angle of incidence. At the same time part of the wave is transmitted into the second medium, whereby the angle of refraction is determined by Snell's law:

$$
\frac{\sin \left(\theta_{i}\right)}{\sin \left(\theta_{t}\right)}=\frac{c_{u s, 1}}{c_{u s, 2}}
$$

where $\theta_{i}$ and $\theta_{t}$ are the angles of incidence and refraction with respect to the normal, respectively, and $c_{u s, 1}$ and $c_{u s, 2}$ are the sound velocities in medium 1 and 2 , respectively. The intensity of the refracted wave part can be both larger and smaller than the intensity of the reflected wave part. This is governed by the acoustic impedances $Z_{1}$ and $Z_{2}$, which relate particle velocity to particle pressure. For a plane wave in a nonattenuating medium, the acoustic impedance has no imaginary component and equals:

$$
Z=\rho \cdot c_{u s} \quad\left[\mathrm{~kg} \cdot \mathrm{m}^{-2} \cdot \mathrm{s}^{-1}\right]
$$

At normal incidence, the intensity $I_{r}$ of the reflected wave and the intensity $I_{t}$ of the refracted wave can be shown to be related to the intensity $l_{i}$ of the incident wave according to: 


$$
\begin{aligned}
& \frac{I_{I}}{I_{i}}=\frac{\left(Z_{2}-Z_{1}\right)^{2}}{\left(Z_{2}+Z_{1}\right)^{2}} \\
& \frac{I_{t}}{I_{i}}=\frac{4 Z_{2} \cdot Z_{1}}{\left(Z_{2}+Z_{1}\right)^{2}}
\end{aligned}
$$

Apparently, if $Z_{2}$ equals $Z_{1}$, no reflection occurs and all of the wave energy is transmitted into medium 2.

A third effect associated with wave propagation through biological material is attenuation: a gradual decrease in wave amplitude as a function of the distance traveled. In fact, attenuation is the cumulative result of a number of separate mechanisms, including the spreading of the wave over a growing volume, the scattering by tiny structures (causing part of the wave energy to be redirected) and absorption, whereby wave energy is converted into heat. The degree of wave amplitude attenuation as a function of the distance $d$ traveled may be expressed as (Wells, 1977):

$$
A(f, d)=A(f, 0) \cdot e^{-\mu(f) \cdot d}
$$

where $A(f, 0)$ is the original amplitude and $\mu(f)\left[\mathrm{Np} \cdot \mathrm{cm}^{-1}\right]$ is the frequency dependent attenuation coefficient. Attenuation models often assume an approximate linear relationship between $\mu(f)$ and $f$ for soft tissue (Cloostermans and Thijssen, 1983; Oosterveld et al., 1991):

$$
\mu(f) \approx \mu_{1} \cdot f
$$

where $\mu_{1}$ is a constant. For pulsed ultrasound, characterized by a nonzero frequency bandwidth, attenuation results in a gradual downward shift of the mean sound frequency with distance. Only in the special case that the wave has a Gaussian amplitude spectrum, the shape of the spectrum, including the bandwidth, is preserved, provided that Eq. (2.8) is applicable (Ferrari and Jones, 1985). Since different types of tissue, including pathological tissues, have different attenuation coefficients, $\mu(f)$ is of importance in tissue characterization (Jones and Leeman, 1984). In literature, the related parameter $\alpha(f)=(20 / \ln (10)) \cdot \mu(f)\left[\mathrm{dB} \cdot \mathrm{cm}^{-1}\right]$ is often used to express attenuation. The mean value of $\alpha(f)$ of soft tissue is of the order of $0.5 \mathrm{~dB} / \mathrm{cm}$ at $1 \mathrm{MHz}$.

Finally, the Doppler effect is mentioned because of its wide application in clini- 
cal blood velocity measurements (Powis and Schwartz, 1991). If a sound wave encounters a moving object, the backscattered wave shows a shift in mean frequency. The relation between the frequency shift $f_{d}\left[\mathrm{~s}^{-1}\right]$ and the object velocity $v\left[\mathrm{~m} \cdot \mathrm{s}^{-1}\right]$ is given by the Doppler equation:

$$
f_{d}=\frac{2 f_{c} \cdot v \cdot \cos (\theta)}{c_{u s}}
$$

where $f_{c}$ is the original sound frequency and $\theta$ is the angle between the directions of wave propagation and object motion. It follows that objects moving in the direction of wave propagation $(\theta=\pi)$ induce a decrease in mean frequency in the backscattered wave, while objects moving in the opposite direction $(\theta=0)$ lead to an increase in mean frequency.

\subsection{Emission and reception}

The device used for both ultrasound emission and reception is the ultrasound transducer, converting electrical energy into mechanical energy and vice versa. In its simplest form, the transducer consists of a single piece of piezoelectric material on which two electrodes are mounted, one on each side of the slice. When an electric pulse is applied to the electrodes, the piezoelectric element will respond with a damped oscillation of its thickness. A continuous mechanical oscillation is achieved by a sinusoidal excitation, matched to the natural resonance frequency of the element. When the front of the transducer is brought into contact with some medium, the oscillations will be radiated as ultrasound into the material, provided that the mutual difference in acoustic impedance is not too large. Conversely, when sound waves backscattered by microscopic inhomogeneities strike the transducer surface, an electric signal is induced.

Two modes of operation are generally distinguished. In continuous wave (CW) mode, the transducer establishes a stationary ultrasound field by continuous radiation of sound waves. In pulsed wave (PW) mode, the transducer generates a transient field by emitting short bursts of ultrasound at regular intervals. The two ways of operation each require a specific transducer design (Bamber and Tristam, 1988). The emission of a short burst of (broad band) ultrasound is only possibly if the mechanical oscillations of the piezoelectric element quickly decay after excitation. To provide for the necessary damping, PW transducers have a backing medium against the rear side of the piezoelectric material (Foster and Hunt, 1978). The shortest sound pulses thus achievable are about one period at the resonance fre- 
quency of the element. The adverse effect of damping is a decrease in sensitivity. For this reason, CW transducers are usually simply backed with air.

In addition to $\mathrm{PW}$ or $\mathrm{CW}$ operation, the character of the sound field produced is determined by the shape of the transducer aperture as well as, for multiple element transducers, the shape of the (complex) aperture weighting function applied (apodization). According to Huygens' principle, the transducer face may be regarded as a composition of many tiny elements, all acting as point sources producing spherical waves. The sound intensity at some location in front of the transducer results from interference of the separate contributions of all point sources. Consequently, close to the transducer, where the differences in path lengths to the different point sources are large with respect to the wavelength, a complex pattern of pressure amplitude maxima and minima can be observed (Zemanek, 1971).

One of the simplest transducers is the unfocused piston transducer, having a circular aperture geometry with a radius a. Analysis of the sound field produced by this transducer in a lossless medium under continuous wave excitation and uniform aperture weighting, shows that most of the acoustical energy is confined within a narrow region or beam. The beam axis, defined as the line connecting the locations of maximum pressure amplitude, is perpendicular to the piston surface and cuts it in the center. In a rough approximation, two regions can be distinguished. In the "near field" or Fresnel zone, close to the transducer, the beam is parallel but inhomogeneous. Its diameter is roughly equal to the transducer diameter. Further away from the transducer, in the "far field" or Fraunhofer zone, differences in path lengths are very small as compared with the wavelength, giving a more homogeneous but diverging beam. The beam divergence is a result of wave diffraction. In the field of optics, the shape of the lateral beam amplitude profile in the far field is known as the Airy amplitude pattern (Goodman, 1968; Steward, 1983). As can be seen in figure 2.1, on both sides of the central main lobe a series of side lobes exists. A useful measure for the beam width in the far field is the fullwidth-at-half-maximum (FWHM) of the lateral beam profile (Foster and Hunt, 1979). For the Airy amplitude profile it can be shown that:

$$
\operatorname{FWHM}_{\mathrm{LBP}}(y) \approx 0.71 \frac{\lambda \cdot y}{\mathrm{a}}
$$

where $y$ is the axial distance to the transducer.

Focusing of the beam in the near field is achieved by using a curved transducer surface, by positioning an acoustic lens in front of the transducer, or by applying 


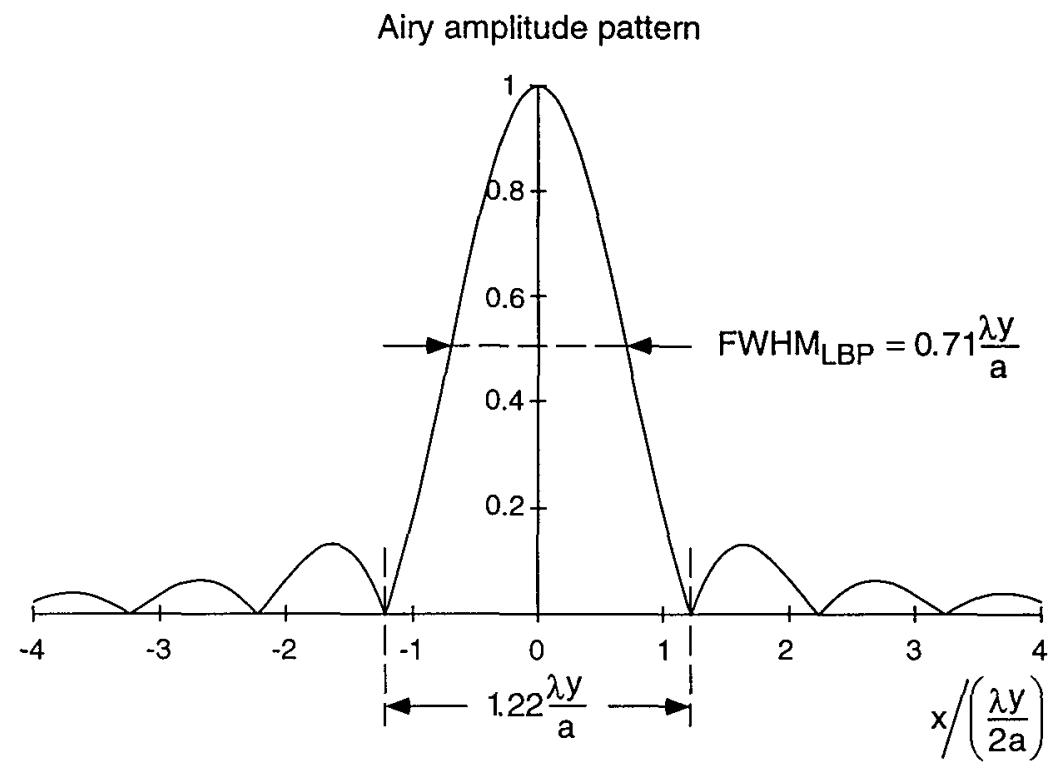

Figure 2.1. Lateral beam amplitude profile in the far field of a piston transducer. Variable a equals the radius of the circular transducer aperture, $\lambda$ is the wavelength, $y$ and $x$ denote axial distance to the transducer and distance perpendicular to the beam axis, respectively.

the following complex aperture weighting function (Lu et al., 1994):

$$
W(r)=e^{-\mathbf{j} \cdot \frac{2 \pi}{\lambda} \cdot\left(\sqrt{F^{2}+r^{2}}-F\right)}
$$

where $r$ is the radial distance of a point source on the circular transducer surface, $F$ is the focus point in front of the transducer and $j=\sqrt{-1}$. Essentially, Eq. (2.11) describes a phase shifter which causes the contributions of all virtual point sources on the transducer surface to arrive in phase in $F$. The phase shifts can be realized in practice by splitting the piston transducer aperture into a number of concentric rings (the so-called annular array configuration), and applying radius-dependent delays to the excitations of the rings. The advantage of this approach of electronic focusing is, that the focal depth $F$ can be flexibly manipulated by changing the delays. The lateral beam width of the focused piston transducer equals:

$$
\mathrm{FWHM}_{\mathrm{LBP}}(\mathrm{F}) \approx 0.71 \frac{\lambda \cdot \mathrm{F}}{\mathrm{a}}
$$

It follows that, for a given focal depth, the focal beam width can be reduced by in- 
creasing the transducer diameter as well as increasing the transducer frequency. Side effects of stronger focusing are increased beam divergence in the far field and shortening of the depth of field around the focus point (i.e., shortening of the distance between the two out-of-focus planes in which the beam width equals $\sqrt{2}$ times the focal beam width) (Lu et al., 1994; Sung, 1989).

An important effect of $\mathrm{PW}$ instead of $\mathrm{CW}$ excitation on the beam profile is, that the interference pattern in the near field becomes less pronounced (Hill, 1986). This is due to the fact that when the emission duration is shortened, the bandwidth of the sound wave is increased. As a consequence, the acoustical field is smoothed since each of the frequencies emitted produces a different pattern of minima and maxima. In this respect, the $Q$ factor is important, relating the central ultrasound emission frequency $f_{c}$ to the FWHM bandwidth $B W$ :

$$
Q \stackrel{\text { def }}{=} \frac{f_{c}}{B W}
$$

Defining the effective pulse emission duration $\tau_{p}$ as the time elapsing between the two moments that the pulse amplitude equals half the maximum level, and assuming the uncertainty relation $\tau_{p} \cdot B W=1$ (Gabor, 1945), the $Q$ factor is found to equal the effective number of periods emitted at $f_{c}$.

So far, the transducer has been regarded as a source of ultrasound. Concerning its behavior as a receiver, it is noted that, for a fixed configuration, the sensitivity of the transducer to echo signals from a certain depth has the same distribution in lateral direction as the local beam amplitude profile generated in emission mode. Consequently, if the same transducer is used for PW ultrasound emission as well as echo reception, the lateral variation of the overall emission/reception sensitivity follows the pattern of the squared lateral beam profile. It follows that the effective width of the local sensitivity region in combined emission/reception mode is smaller than the local beam width (Hunt et al., 1983).

The reason why most ultrasound techniques, including B-mode echo imaging and color velocity imaging, employ $\mathrm{PW}$ instead of $\mathrm{CW}$ ultrasound is, that only the former allows (to a certain extent) the origins of the echoes to be localized along the beam axis. To do so, the PW ultrasound system is switched to reception mode after emission of a short burst of ultrasound, to catch the echoes produced by the tissue insonified. The larger the distance between the transducer and a scattering structure, the longer it takes before the echo is received. Assuming the sound velocity $c_{u s}$ to be a constant, the time $t$ elapsing between ultrasound emission and reception is 
directly proportional to the distance $d$ of the structure:

$$
\mathrm{d}=\frac{\mathrm{cus}_{\mathrm{us}} \cdot \mathrm{t}}{2}
$$

Because the ultrasound pulse emitted has a finite length, and the local, lateral beam profile has a finite width, the instantaneous signal received is a composition of the contributions of all scattering structures located within a clearly defined region: the (echo) sample volume. During the reception of the echoes, the sample volume moves away along the transducer axis at half the speed of ultrasound. The axial length of the sample volume may be defined as the effective pulse duration multiplied by $c_{u s} / 2$ :

$$
S V_{\mathrm{ax}}=\frac{\mathrm{C}_{\mathrm{us}} \cdot \tau_{\mathrm{p}}}{2}
$$

while the lateral width of the sample volume is limited by the local width of the sensitivity region. Echoes from scatterers whose mutual distance is smaller than the local dimensions of the sample volume, cannot be completely separately resolved, explaining why the sample volume is also called resolution cell.

Taking into account beam diffraction and attenuation effects, the overall process relating the electric excitation of the transducer to its final electric response may be modeled as (Hottier and Bernatets, 1984):

$$
r(t)=\sum_{i} e(t) \otimes i_{t}(t) \otimes h\left(r_{i}, t\right) \otimes a t t\left(r_{i}, t\right) \otimes u_{b s}\left(r_{i}, t\right) \otimes a t t\left(r_{i}, t\right) \otimes h\left(r_{i}, t\right) \otimes i_{r}(t)
$$

where $r f(t)$ is the electric (radio frequency) signal received, $e(t)$ is the electric excitation of the transducer, $i_{t}(t)$ and $i_{r}(t)$ are the acousto-electric and electro-acoustic responses of the transducer, respectively, $h\left(r_{i}, t\right)$ is the diffraction impulse response, accounting for the effect of wave diffraction, att $\left(r_{i}, t\right)$ is a function accounting for the effect of attenuation, and $u_{b s}\left(\boldsymbol{r}_{i}, t\right)$ is a function describing the backscattering characteristics of a scatterer located at point $\boldsymbol{r}_{i}$. The symbol $\otimes$ denotes convolution. In ultrasonic imaging, the backscattering properties of the object insonified are of primary interest. However, as indicated by Eq. (2.16), the relevant information, embodied in $u_{b s}\left(\boldsymbol{r}_{i}, t\right)$, is not readily available. Nevertheless, the rf signal has turned out to be a valuable source of information in many medical applications. 


\subsection{B-Mode echo imaging}

Consider an ultrasound pulse striking a single scattering object. The strength of the echo produced is determined by the acoustic impedance mismatch of the object in relation to its environment. Hence, the amplitude level of the corresponding rf signal too is related to the scatter strength. In addition, the distance of the object (its depth) is related to the time elapsing between pulse emission and echo reception, as described by Eq. (2.14). In brightness mode or B-mode echo imaging, these two principles are employed to construct a two-dimensional image of a planar section of a structure investigated. In this method, the object is interrogated along a number of separate scan lines (i.e., beam axes) which together make up the plane of observation. Along each scan line, an ultrasound pulse is emitted and the resulting rf signal recorded. Depending on whether the scan line is gradually rotated or shifted, the area covered has the shape of a slice of pie (sector scan) or a rectangle (linear scan), respectively. In its most basic form, the B-mode image is created while scanning, by directing an electronic spot over a CRT screen along with the movement of the sample volume, and by modulating the luminance of the spot with the instantaneous amplitude of the rf signals. Continuous repetition of the scan process gives a cinematographic effect, enabling moving structures to be visualized in real time. The spatial resolution of a B-mode echo system can be defined as the reciprocal of the minimum separation two point scatterers must have in order to be distinguishable on the screen. An equivalent definition, more convenient in practice, is that the spatial resolution is equal to the reciprocal of the size of the spot on the screen produced by a single point scatterer (Harris et al., 1991). It follows that the resolutions in axial and lateral direction (perpendicular to the beam) correspond to the effective sizes of the sample volume in the two directions.

In modern ultrasound systems, the whole process of image construction is carried out digitally. A possible configuration is shown in figure 2.2. For the sake of simplicity, the control section, providing the necessary coordination between the different system parts, has been left out. The central section consists of a shared memory via which the various blocks exchange data. The vertical line of blocks is recognized as the data acquisition part, supplying the memory with digitized rf signals, while the horizontal line represents the data processing and displaying part, converting a set of $\mathrm{rf}$ signals into a B-mode image. The transducer is designed to enable a rapid redirection of the scan line in the plane of observation. Rotation of the scan line is either achieved by mounting an annular array on a rotating wheel (mechanical sector scanner) or by using the phased array transducer configuration (electronic scanner). In the latter approach, the transducer aperture is build up of a 


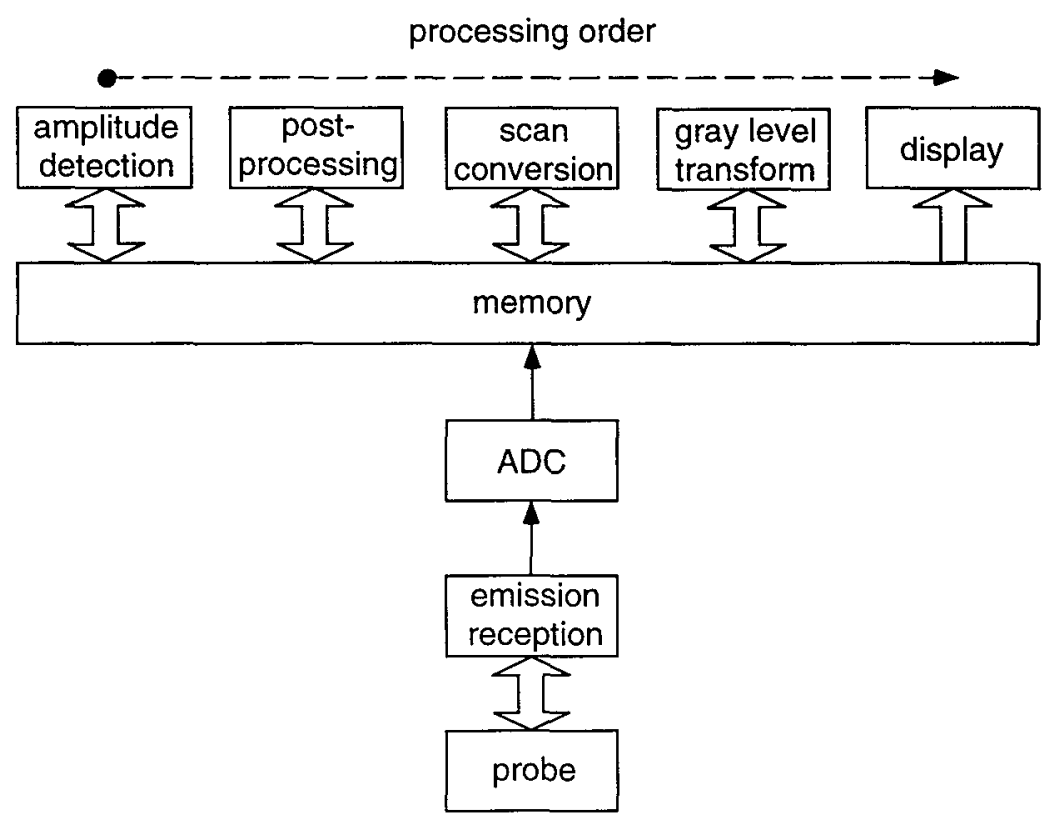

Figure 2.2. Block scheme of an advanced B-mode echo imaging device, with exclusion of the control section. The vertical line of blocks represents the data acquisition part, the horizontal line the data processing and displaying part. The dashed arrow indicates the order of data processing.

small, one-dimensional array of elements. Analog to the focusing of the annular array, the beam angle can be steered by applying appropriately delayed excitations to the elements. The option of scan line translation is offered by the linear array transducer, which differs from the phased array in that it contains a multiple number of elements. The principle is, that a focused beam is generated by addressing a group of neighboring elements, and that the beam is gradually shifted along the linear array by successively addressing different but overlapping groups.

The emission/reception block provides the excitation of the transducer and the amplification of the transducer's electric response to echo signals. To correct for the attenuation effect, the receiver amplifier is equipped with a variable time gain compensation (TGC): while recording an rf signal, the gain is gradually increased so that the weak echoes of remote structures are stronger amplified than the less attenuated echoes of close structures. TGC is intended to make, for a given scattering object, the signal strength independent of the depth of the object. The rate at which ultrasound pulses can be emitted (i.e., the pulse repetition frequency or $P R F\left[\mathrm{~s}^{-1}\right]$ ) is 
primarily limited by the depth of investigation. To avoid ambiguity of location, the echo response of the object interrogated should have died out before another pulse can be emitted. A practical limit is:

$$
\mathrm{PRF}_{\max }=\frac{\mathrm{c}_{\mathrm{us}}}{2 \mathrm{~d}_{\max }}
$$

where $d_{\max }$ is the maximum depth of investigation. For a frequency dependent attenuation of $1 \mathrm{~dB} / \mathrm{MHz}$ per centimeter round trip and a maximum attenuation of 40 $\mathrm{dB}, d_{\max } \approx 8 \mathrm{~cm}$ at a transducer frequency of $5 \mathrm{MHz}$, and $P R F_{\max } \approx 10 \mathrm{kHz}$. The $\mathbf{r f}$ signals collected during a single scan of the plane of observation make up a frame. The maximum frame rate $F R_{\max }\left[\mathrm{s}^{-1}\right]$ equals:

$$
\mathrm{FR}_{\max }=\frac{\mathrm{PRF}_{\max }}{\mathrm{n} \ln \mathrm{s}}
$$

where nIns is the number of scan lines per frame.

The analog-to-digital converter (ADC) on-line digitizes the rf signals. Its dynamic range (i.e., the maximum output expressed in units of the minimum nonzero output) should be large enough to prevent, on the one hand, high amplitude signals (from strong specular reflectors) from clipping, and, on the other hand, low amplitude signals (from weak scatterers) from drowning in the quantization noise. After TGC, the dynamic range of the signals is of the order of 50 $\mathrm{dB}$, so that a $9+1$ bits $\mathrm{ADC}$ is sufficient. In accordance with the Nyquist theorem, the sampling frequency $f_{s}$ should be at least twice the maximum signal frequency. Taking into account that the ultrasound $Q$ factor is normally larger than 1 , a practically suitable value of $f_{s}$ is 4 times the central ultrasound emission frequency:

$$
f_{s}=4 f_{c}
$$

To avoid axial position jittering of the sample volume over the consecutive digital $\mathrm{rf}$ signals, the sample clock should be synchronized with the ultrasound emission trigger. It is noted that hybrid systems, employing analog amplitude detection and postponing $\mathrm{AD}$ conversion up to the block "postprocessing", are less demanding as regards the sampling frequency $f_{s}$.

\subsubsection{Amplitude detection}

The rf signal amplitude at $t=n \cdot \tau_{s}\left(\tau_{s}=1 / f_{s}, n\right.$ is an integer $)$ is calculated as 


$$
A\left(n \tau_{s}\right)=\sqrt{r^{2}\left(n \tau_{s}\right)+r_{Q}^{2}\left(n \tau_{s}\right)}
$$

where $f_{Q}\left(n \tau_{s}\right)$ is the quadrature (90 degrees phase shifted) rf signal. This signal can be calculated exactly using the Hilbert transform: in the frequency domain, the negative part of the spectrum of $r\left(n \tau_{s}\right)$ is set to zero while the positive part is multiplied by a factor 2 (to preserve signal power). As a result, the corresponding complex time domain signal has a real part $f\left(n \tau_{s}\right)$ and an imaginary part $f_{Q}\left(n \tau_{s}\right)$. The drawback of this procedure is, that it is rather cumbersome, making it not very suitable for implementation in real-time imaging systems.

An alternative approach, computationally more attractive while still accurate, is to apply a FIR approximation of the Hilbert transformer in the time domain. The signal $r f_{Q}\left(n \tau_{s}\right)$ is theoretically obtained by convolving $r f\left(n \tau_{s}\right)$ with the series (Rabiner and Gold, 1975):

$$
h_{\text {Hiib }}\left(n \tau_{s}\right)= \begin{cases}2 /(n \cdot \pi) & |n| \text { is odd } \\ 0 & \text { otherwise }\end{cases}
$$

As this series is doubly infinite in extent, it has to be approximated in practical realizations by a finite series, by multiplying $h_{\text {Hilb }}\left(n \tau_{s}\right)$ with some window function centered around $n \tau_{s}=0$. The resulting FIR time domain Hilbert transformer performs best if spectral signal power is centered in the middle of the frequency domain available, which is true for typical rf signals sampled at about four times the central ultrasound emission frequency.

Another alternative, attractive because of its exceptional simplicity, requires the sample frequency $f_{s}$ to equal four times the central ultrasound emission frequency $f_{c}$. The quadrature signal may then be approximated by $r f_{Q}\left(n \cdot \tau_{s}\right) \approx$ $f\left((n+1) \cdot \tau_{s}\right)$. The smaller the rf signal bandwidth, the better the approximation. Note that this procedure yields an amplitude signal that is shifted over $\tau_{s} / 2$ as compared with the exact amplitude.

The above two alternative approaches to amplitude detection were compared with the exact method in a small simulation study. Five noise signals were generated in the frequency domain. Noise power was concentrated in a rectangular band around zero frequency. The bandwidth ranged in equal steps from $0.05 f_{s}$ for the first signal up to $0.25 f_{s}$ for the last one. The spectra within these base bands were filled by randomly pulling, for each frequency involved, a real and imaginary part from the 


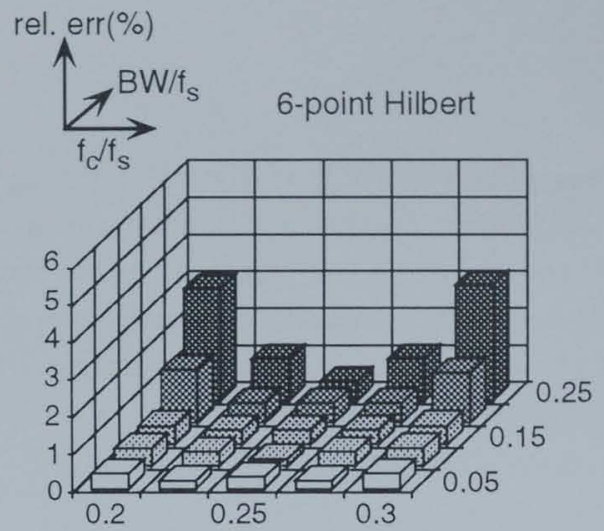

(a)

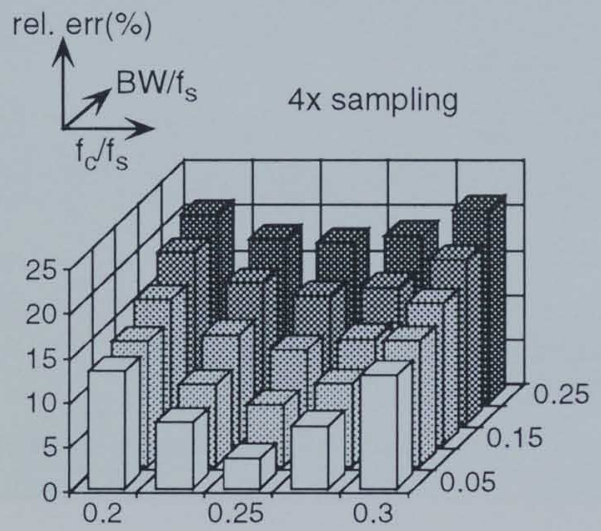

(c) 6-point Hilbert, 4-point MA filter

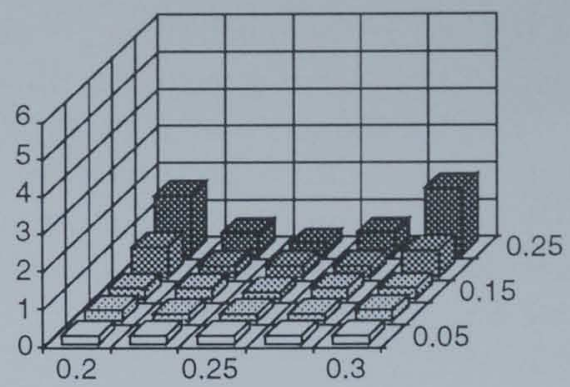

(b)

\section{4x sampling, 4-point MA filter}

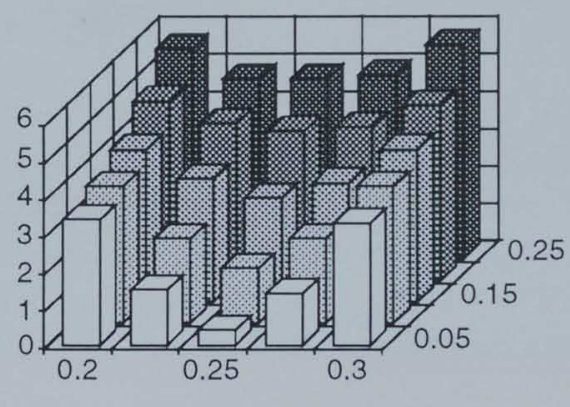

(d)

Figure 2.3. Evaluation of amplitude detection on the basis of a 6-point time domain Hilbert transformer (a), and on the basis of fourfold sampling, whereby adjacent rf samples are treated as quadrature pairs (c). Each bar indicates the standard deviation of the error signal relative to the mean amplitude level for a particular combination of the normalized ultrasound emission frequency, $f_{C} / f_{S}$, and the normalized ultrasound bandwidth $B W / f_{S}$. The error signal is calculated as the approximated amplitude signal minus the exact amplitude signal. Figures (b) and (d) show the results if all amplitude signals are smoothed by a 4-point MA filter prior to the calculation of the error signals. 
standard normal distribution. Inverse Fourier transformation yielded five complex, time domain noise signals. The center frequency $f_{c}$ could be varied by modulating the noise signals with a complex harmonic signal. This way, 25 complex rf signals were generated, divided over 5 different center frequencies, ranging in equal steps from $0.20 f_{s}$ to $0.30 f_{s}$. The two algorithms for calculating $f_{Q}(t)$ were applied to the real parts of all signals. The time domain Hilbert transformer was derived from Eq. (2.21) by multiplying $h_{\text {Hilb }}\left(n \tau_{s}\right)$ with a Hanning window. The resulting kernel contained 6 nonzero values. The time shifts induced by the second alternative method (based on $f_{s}=4 f_{c}$ ) were eliminated by means of harmonic interpolation. As a measure of performance was used the relative standard deviation of the error signal, defined as the approximated amplitude signal minus the reference amplitude signal. The results are presented in figure 2.3. Figure 2.3-a and -c show, for each of the two methods, the standard deviations of the error signals relative to the mean amplitude levels as a function of the $\mathrm{rf}$ center frequency $f_{C}$ and the $\mathrm{rf}$ signal bandwidth $B W$. Obviously, for all signal conditions the time domain Hilbert transformer performs superior to the rough approach of assuming quadruple sampling. The latter method is only acceptable if $f_{s}$ is indeed close to $4 f_{c}$ and $B W$ is at the same time small with respect to $f_{c}$. However, a substantial reduction of the relative errors as produced (in particular) by the method of fourfold sampling (Figs. 2.3-b and $-\mathrm{d}$ ) is achieved by applying a 4-point arithmetic mean filter to the reference envelopes as well as to the estimates before calculating the error signals. It is noted that the moving average filter hardly influences resolution since the window length used is always smaller than the ultrasound pulse length associated with the signal $\left(\approx Q \cdot f_{S} / f_{C} \approx f_{s} / B W\right.$ sample points, in accordance with Eq. (2.13)). The conclusion is that envelope signals calculated with a 6 -point time domain Hilbert transformer closely approximate the actual signals, for narrow band as well as broad band $\mathrm{rf}$ signals, and especially if $f_{s}=4 f_{c}$. The other method in which pairs of adjacent $\mathrm{rf}$ samples are combined to calculate amplitude is only reasonably accurate over a wide variety of signal conditions if the estimated envelope signals are smoothed afterwards, which makes this method loose its elegance.

\subsubsection{Scan conversion}

In ultrasound B-mode imaging, echo information is obtained on the base of a two-dimensional spatial coordinate system determined by the ultrasound scanner. Sector scanners produce data in a polar coordinate system, while linear array transducers use a rectangular grid. To enable the display of all information by a video monitor, the distributions measured are transformed into rectangular grids of pixels by a digital scan converter (DSC), which is essentially a coordinate transformer. In the theoretically optimal conversion method, $2 \mathrm{D}$ sampling theory is 

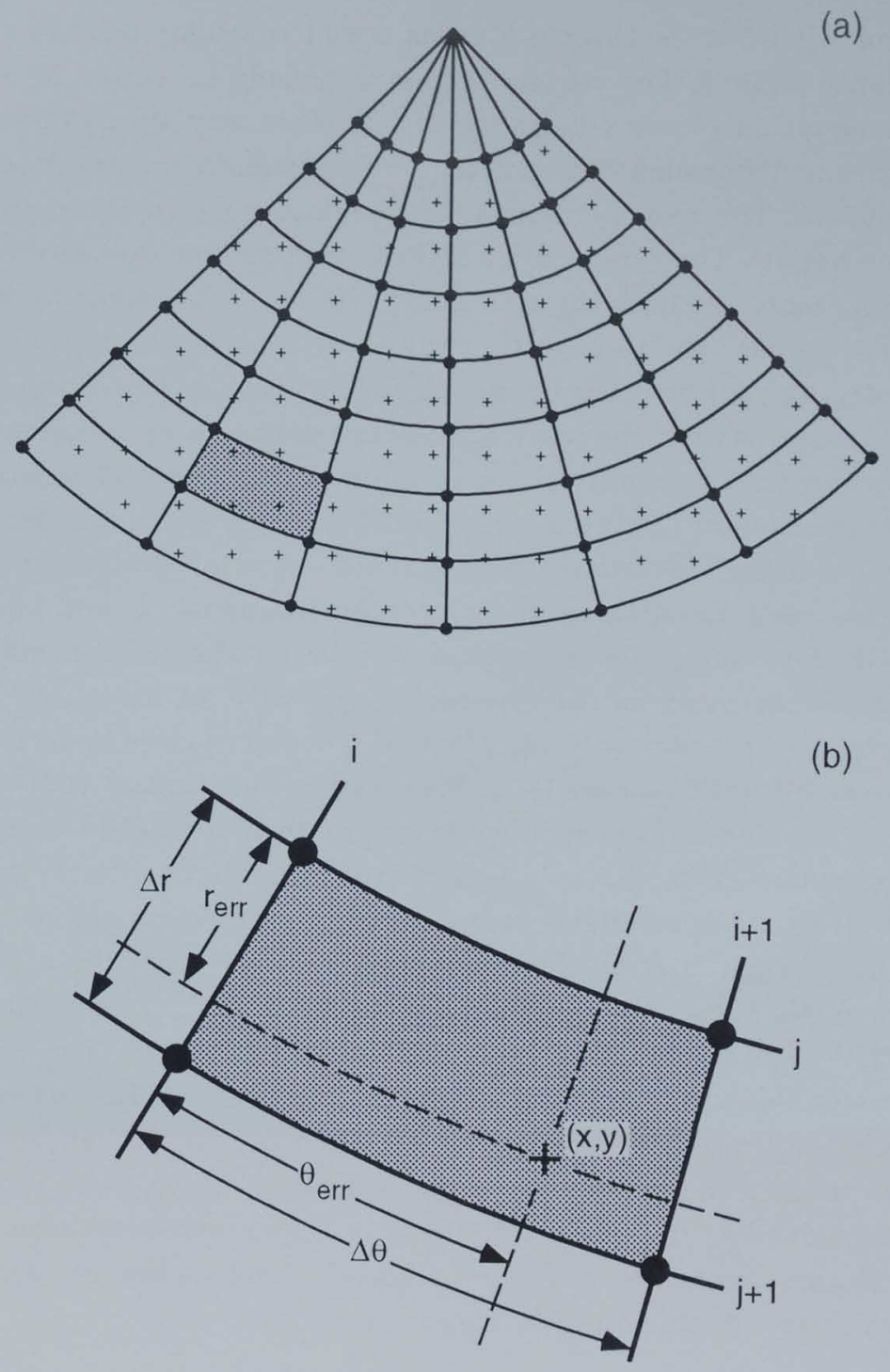

(b)

Figure 2.4. Bilinear scan conversion. (a) Data points produced by a sector scanner (with polar coordinates) are superimposed on the pixel space (with Cartesian coordinates). Data points are indicated by dots, pixels by the + sign. (b) Close-up of the gray area in (a). A pixel value at location $(x, y)$ in the pixel space is calculated by bilinear interpolation over the four data points surrounding the pixel. Variables $i$ and $j$ denote scan line number and data point number, respectively, and $r$ and $\theta$ denote radial distance to the transducer and scan angle, respectively. 
applied to reconstruct the continuous signals out of the sampled data, whereafter the pixel values are found by resampling the continuous distribution. However, due to the high computational cost, this method is of limited importance in real-time applications. Various approximate methods have been described, exchanging accuracy of conversion for speed of operation (Lee et al., 1986; Lee and Park, 1985; Ophir and Maklad, 1979; Parker et al., 1983; Richard and Arthur, 1994; Robinson and Knight, 1982).

The bilinear interpolator (Leavitt et al., 1983) has been shown (Berkhoff et al., 1994) to be the best compromise, both objectively and subjectively, between conversion error and computation time, especially if the implementation is based on lookup tables. It superimposes the data points on the spatial distribution of pixels, as depicted in figure 2.4-a for a sector scanner, and divides the input distribution into sample quadrants. Each pixel value pix $(x, y)$ is calculated by performing a bilinear interpolation over the 4 data points marking the corners of the quadrant enclosing the pixel (see Fig. 2.4-b). The interpolation is formally described by:

$$
\begin{aligned}
& s_{1}=\left(1-\frac{r_{\text {err }}}{\Delta r}\right) \cdot s(i, j)+\frac{r_{\text {err }}}{\Delta r} \cdot s(i, j+1) \\
& s_{2}=\left(1-\frac{r_{\text {err }}}{\Delta r}\right) \cdot s(i+1, j)+\frac{r_{\text {err }}}{\Delta r} \cdot s(i+1, j+1) \\
& \operatorname{pix}(x, y)=\left(1-\frac{\theta_{\text {err }}}{\Delta \theta}\right) \cdot s_{1}+\frac{\theta_{\text {err }}}{\Delta \theta} \cdot s_{2}
\end{aligned}
$$

where $s(i, j)$ indicates the value of the $j^{\text {th }}$ data point on scan line $i$, and $r_{\text {err }}$ and $\theta_{e r r}$ are the radial and angular direction errors, respectively, as indicated in figure 2.4b. Operation becomes faster if the quadrants are located in advance for each pixel, and all factors $r_{e r r} / \Delta r$ and $\theta_{e r r} / \Delta \theta$ are precalculated. Recalculations are only required for rescaling the image. For linear array systems, calculations are analog, but with the factors $r_{e r r} / \Delta r$ and $\theta_{e r r} / \Delta \theta$ replaced by $y_{e r r} / \Delta y$ and $x_{e r r} / \Delta x$, respectively.

\subsubsection{Gray level transform and display}

The rectangular grid of pixels produced by the DSC is presented to the user as a brightness image on a video monitor. Thereby, a one-on-one relation exists between pixel value and luminance or gray level of the corresponding picture element on the screen. The character of the dependence is partly determined by the properties of the monitor. Generally, the transfer function between monitor steering level and 
screen luminance is a power function, typical values of the power or "gamma" ranging between 2 and 3 . In addition, the relation pixel value - gray level depends on the gray level transform applied to each element in the output distribution of the DSC. The gray level transform, which may be implemented as a look-up table, serves two goals. In the first place, it performs range compression, adapting the large dynamic range of the echo amplitudes to the much smaller range the monitor can handle. Typically, the echo dynamic range is about $50 \mathrm{~dB}$ while the input signal of the monitor is encoded in 6 to 7 bits, corresponding to a dynamic range of 36 to 42 dB. Commonly, compression curves are based on a logarithmic function. Application of an $\mathrm{S}$ shaped curve can be profitable, as this avoids strong amplification of the noise level.

In the second place, gray level transforms were demonstrated to influence the first- and second-order statistics of the output distribution of the DSC, this way offering the opportunity to optimize the detectability of contrasting regions in the image (Thijssen et al., 1988).

\subsubsection{Postprocessing}

The application of a gray level transform to improve the visibility of contrasting structures in the image (such as focal lesions), may be regarded as a form of signal postprocessing. In general, postprocessing encompasses all additional processing of the signals at some point in the chain between raw rf data and video image, to compensate for the limitations associated with B-mode echo imaging. On the one hand, these limitations are related to the fact that the backscattering properties of the object investigated are obscured by the mechanism of interrogation, conform Eq. (2.16). On the other hand, since the final image is to be interpreted by the human observer, the limitations should be viewed in connection with the properties of the human visual system. This subject is further dealt with in Chapter 3.

The position of the block "postprocessing" in figure 2.2 indicates that the pertinent echo system applies some postprocessing technique to the envelope detected signals. The output consists of a (two-dimensional) frame of processed amplitude points. Normally, the input-output relation is more complicated than the one-onone relation of the gray level transform described above. The value of a point in the output distribution is usually calculated on the basis of a selection of points in the input distribution. If the input points always differ in only one and the same coordinate (either axial position, or scan line, or frame number), the processing is essentially one-dimensional. A typical example is the algorithm for postprocessing color velocity distributions, to be presented in Chapter 6 . The echo amplitude 
processing algorithms of Chapters 4 and 5 are two-dimensional, basing each output frame on a single input frame.

Since the ideal DSC is merely a coordinate transformer, the block "postprocessing" may be inserted directly after the DSC as well. In that case, all processing is applied to the pixel distributions. Factors to be considered in this respect are the possible influence of conversion errors introduced by the actual (nonideal) DSC on the result of processing, the change in information density induced by the DSC, and the difference in computational cost between processing the amplitude distributions and processing the pixel distributions.

\subsection{Blood velocity measurement}

PW ultrasound allows the velocity of moving structures locally to be estimated. In particular blood velocity is of interest in medical diagnosis, although growing attention is paid to the assessment of arterial wall motion during the cardiac cycle. As an introduction to real-time color velocity imaging, in which color encoded blood velocity profiles are superimposed on B-mode echo images, the principles and difficulties of ultrasonic blood velocity measurement are discussed.

Suppose a single point scatterer travels with a constant velocity $v_{y}$ along the beam axis of a PW transducer. The movement of the particle can be tracked by emitting short bursts of ultrasound at intervals of $\tau_{P R F}=1 / P R F[s]$, and recording the corresponding rf signals. Figure 2.5 gives a one-dimensional model of the situation. Each ultrasound pulse consists of 3 periods at the emission frequency and is assumed to have a rectangular envelope. This implies that the instantaneous (echo) sample volume has a rectangular sensitivity distribution along the beam axis, with length $c_{u s} \cdot \tau_{\rho} / 2$. As illustrated in figure 2.5 , the time elapsing between pulse emission and echo reception decreases as the scatterer approaches the transducer. The instantaneous velocity of the scatterer is straight forward calculated as $v_{y}=$ $c_{u s} \cdot \Delta t_{y} \cdot P R F / 2$, where $\Delta t_{y}$ is difference in position between, e.g., the rising slopes in 2 consecutive rf signals. (The subscript in $t_{y}$ is used to distinguish the time progress along the $\mathrm{rf}$ line from the time $t_{v}$ progressing between different $\mathbf{r f}$ signal recordings. In fact, in PW ultrasound techniques, a single rf signal is considered to be an instantaneous reflection of the target status, whereby $t_{y}$ is interpreted only as a measure of axial distance). Thus, velocity estimation of a single particle is possible and straight forward.

In practice, however, where the local, instantaneous blood velocity is of interest, 

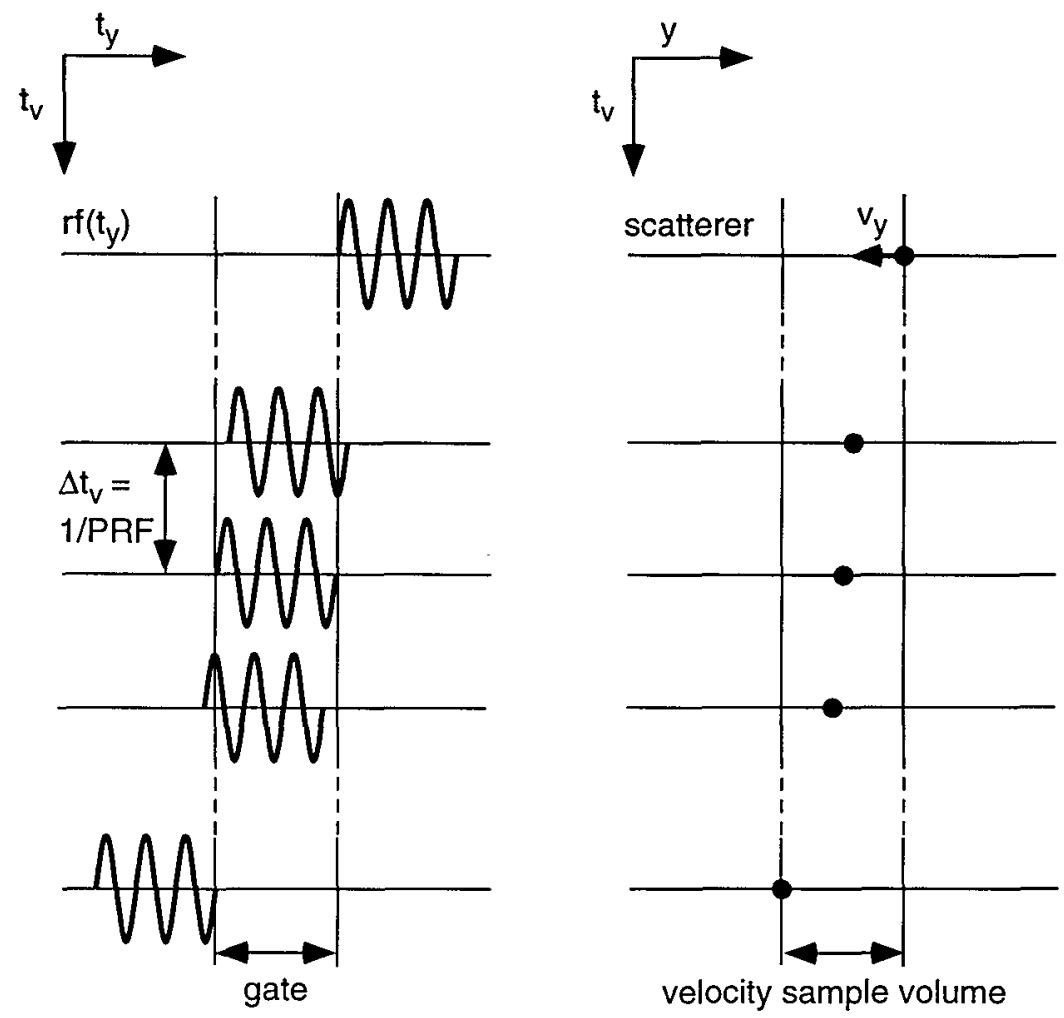

Figure 2.5. Principle of velocity estimation of a target moving along the beam axis of a PW ultrasound transducer. A single point scatterer, indicated by the dot in the right part, can be tracked by emitting ultrasound pulses at regular intervals of $1 /$ PRF [s] and recording the corresponding $\mathrm{rf}$ signals (left part).The rising slope in the $\mathrm{rf}$ signals shifts in accordance with the change in axial position of the particle. In contrast, if a large concentration of moving point scatterers is present, tracking of individual particles is no longer possible. Instead, by investigating the progress of the consecutive rf signal recordings within a fixed window, indicated by gate, a certain group average velocity can be estimated of scatterers moving within a restricted region: the velocity sample volume. Variables $t_{y}$ and $t_{V}$ denote time progress along a single $\mathrm{rf}$ signal and between different $\mathrm{rf}$ signal recordings, respectively, and $y$ denotes axial distance to the transducer. $v_{y}$ is the axial velocity of the single point scatterer. The ultrasound pulses consist of three periods at the central ultrasound emission frequency and have rectangular envelopes.

the situation is more complicated. Instead of a single target, a large distribution of red blood cells is present and, depending on the (time varying) blood flow profile in the vessel, a wide variety of velocities and velocity directions may be encountered. Because the sample volume has finite dimensions, many blood cells contribute at 


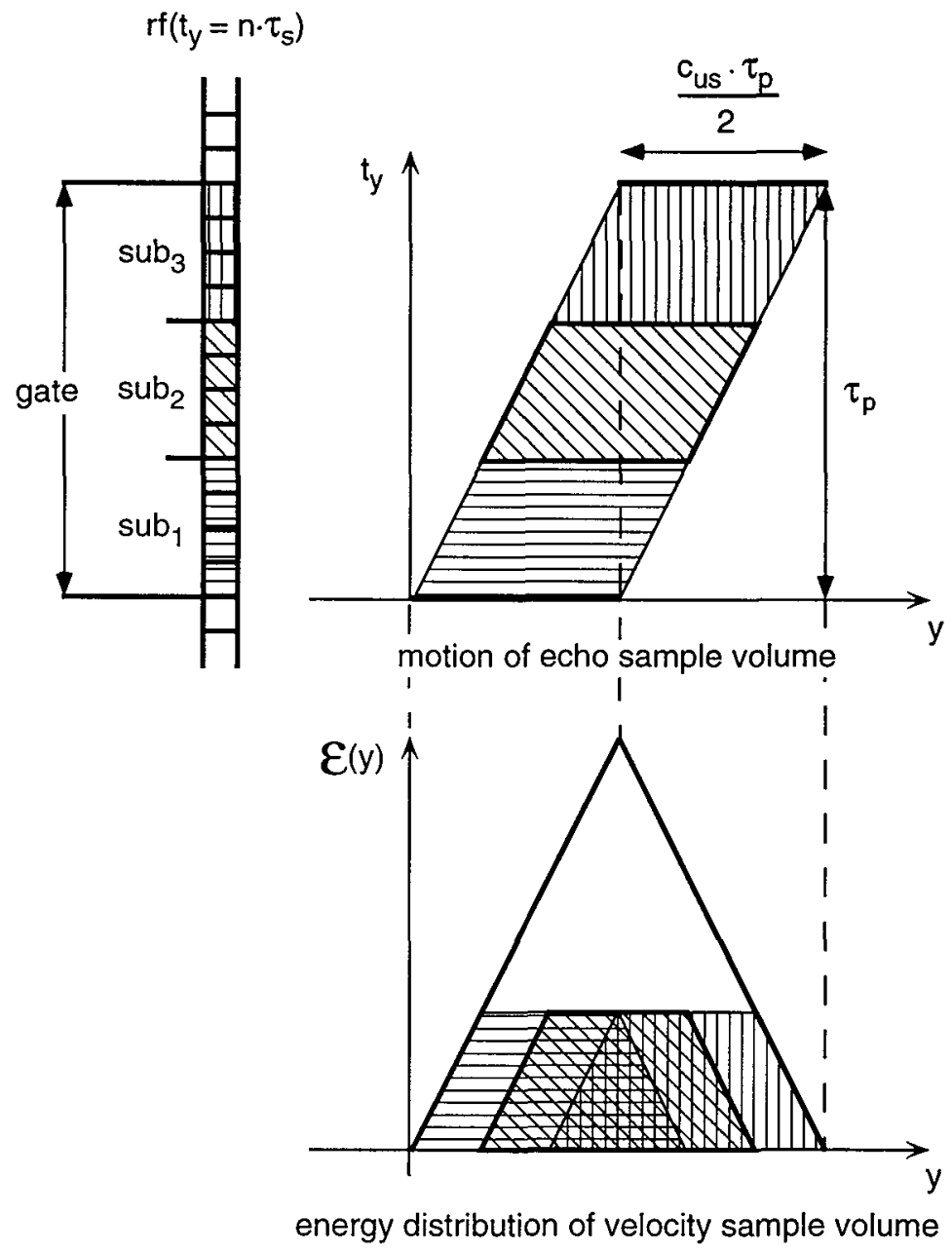

Figure 2.6. Echo sample volume and gate determine velocity sample volume. The upper right shows a graph of the movement of a rectangular (echo) sample volume as a function of $t_{y}$. The initial location of the sample volume is indicated by the bold line on the $y$ axis. After a time $\tau_{p}$, the sample volume has covered a distance equal to its own length. The contribution of a location on the beam axis to the rf signal segment in the uniformly weighted gate (upper left) is proportional to the time that the location is enclosed by the echo sample volume. The result is a velocity sample volume with a triangular energy distribution along the beam axis (bottom). The gate includes twelve sample points which can be grouped into three subgates of four points. The hatch patterns indicate for each subgate the positions of the echo sample volume and the shape of the corresponding velocity subsample volume. 
the same time to the rf signal and individual cells cannot be tracked. As a consequence, one is restricted to estimating the average axial velocity of a group of red blood cells at a certain average depth. To do so, the progress of the consecutive rf signals is investigated within a fixed window, indicated as gate in figure 2.5. The consecutive signal segments in the gate all originate from the same region in the object investigated: the velocity sample volume. According to Eq. (2.14), the position and width of the gate in the rf signals determine the depth and axial length of the velocity sample volume, respectively. As illustrated in figure 2.6, different locations in the velocity sample volume contribute to a different extent to the signal segment in the gate. At the upper right, a graph is shown of the movement of the (echo) sample volume as a function of $t_{y}$. The initial location of the sample volume is indicated by the bold line on the $y$ axis. After a time $\tau_{p}$, in the example corresponding to the gate width, the sample volume has covered a distance equal to its own length. Clearly, the contribution of a location on the scan line to the signal segment in the gate is proportional to the time that the location has been enclosed by the echo sample volume, which can be directly extracted from the graph. The result is a velocity sample volume with a triangular energy distribution along the beam axis, as shown in the lower right of figure 2.6. It is noted that the triangular shape stems from the particular combination of a rectangular echo sample volume with a rectangular gate of width $\tau_{p}$. In general, the velocity sample volume is constructed by convolving a three-dimensional echo sample volume with a (not necessarily rectangular) gate weighting function (Peronneau et al., 1974). Further, it is pointed out that considerations concerning signal-to-noise ratio and resolution have led to the general agreement that $\tau_{p}$ is a good choice of the gate width (Kristoffersen, 1986). Recent findings, however, indicate that a value smaller than $\tau_{p}$ can be profitable (Hoeks et al., 1994). Especially, as will be discussed in subsection 2.4.1.4, the gate may be split up into a number of subgates, each with a duration of one period at the central emission frequency. The different hatch patterns in figure 2.6 indicate for each subgate the positions of the echo sample volume and the shape of the corresponding velocity subsample volume.

Once a set (= package) of $\mathrm{rf}$ signals has been recorded and a gate has been selected, velocity may be estimated. An established and widespread method is the Doppler shift technique, relating the consecutive rf segments to a harmonic reference signal (Burns, 1987; Evans et al., 1989). More recent are rf cross correlation techniques, relating the signal segments to each other instead of a reference signal (De Jong et al., 1990; Foster et al., 1990; Hein et al., 1993; Hein and O'Brien Jr., 1993). 


\subsubsection{Doppler processing}

Figure 2.7 shows the Doppler processing scheme. Input is a package of $\mathrm{rf}$ signal segments, output are a velocity estimate and one or more signal parameter values allowing to determine whether the velocity estimate actually reflects blood velocity. The necessity of the signal parameters follows from the fact that on forehand the nature of the target enclosed by the velocity sample volume is unknown (see section 2.4.1.3). The first step in the Doppler processing scheme consists in calculating, for each signal segment in the package, the average phase difference with respect to a harmonic reference signal with a frequency equal to the central ultrasound emission frequency. Particle motion in the velocity sample volume becomes manifest as a phase gradually changing over the consecutive if

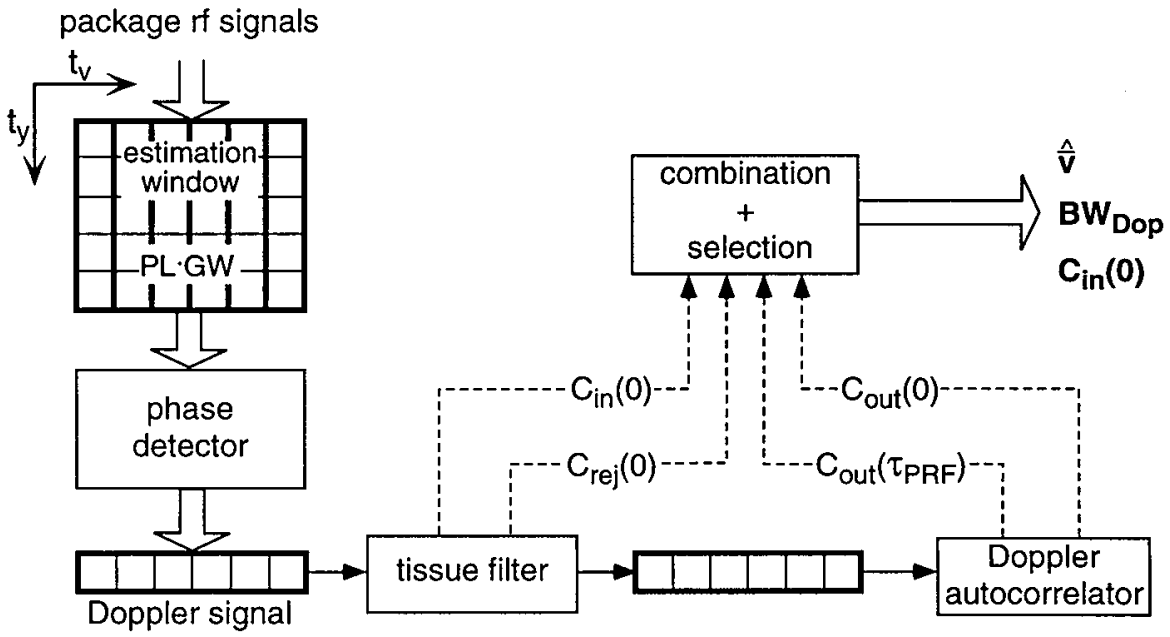

Figure 2.7. Doppler processing scheme. A package of $P L$ consecutive rf signal segments of $G W$ sample points is fed to a phase detector, which calculates for each segment separately a complex point $z$, having an argument equal to the average phase of the rf signal segment and a modulus equal to the average amplitude. The resulting $P L$ complex points make up the Doppler signal. The average Doppler frequency is directly related to the average axial particle velocity in the velocity sample volume. Since blood velocity is of interest, a high pass filter is applied to reject the strong stationary tissue component that may be present. Finally, the filtered Doppler signal is fed to an autocorrelator that calculates the complex autocorrelation coefficients $C_{\text {out }}(0)$ and $C_{\text {out }}\left(\tau_{\mathrm{PRF}}\right)$. On the basis of these coefficients, supplemented with the power of the raw Doppler signal, $C_{i n}(0)$, and the power of the tissue component rejected, $C_{r e j}(0)$, an average velocity $\hat{\bar{v}}$ is estimated as well as some parameter values that allow to determine whether $\hat{\bar{v}}$ actually reflects blood velocity, such as the bandwidth of the filtered Doppler signal, $B W_{D o p}$. 
segments. The rate of change is proportional to the average particle velocity. The phase detector in figure 2.7 calculates for each of the rf segments a complex point, the argument of which is equal to the average instantaneous phase while the modulus equals the average rf signal amplitude in the gate. The output $z$, based on a single rf segment under uniform gate weighting, is calculated as:

$$
z=\frac{2}{G W} \sum_{n \tau_{s} \in \text { gate }} \mathrm{rf}\left(\mathrm{n} \tau_{\mathrm{s}}\right) \cdot \mathrm{e}^{-\mathrm{j} \cdot 2 \pi \mathrm{f}_{\mathrm{c}} \cdot n \tau_{\mathrm{s}}}
$$

where $f\left(n \tau_{s}\right)$ is the value of the rf signal sampled at $t_{y}=n \cdot \tau_{s}\left(\tau_{s}=1 / f_{s}\right)$, the complex exponent is the reference signal (with $j=\sqrt{-1}$ ) and $G W$ is the gate width expressed in number of sample points. Eq. (2.23) essentially describes a cross correlation of the rf signal segment with the reference signal. In practical implementations, the proportionality factor $2 / G W$ may be left away. Furthermore, if $t_{s}$ is set to $4 f_{c}$, the reference signal may be reduced to the repeated sequences $\{1,-1,-1,1\}$ (real part) and $\{-1$, $-1,1,1\}$ (imaginary part), so that only sign operations and summations remain in the calculation of $z$. Subsequent phase detection to all $\mathrm{rf}$ segments in the package gives $P L$ complex points ( $P L$ is the package length, i.e. the number of rf signals in the package) which together make up the Doppler signal $z\left(i \cdot \tau_{P R F}\right)$, where $0 \leq i<P L$. The Doppler signal owes its name to the fact that the average rate of phase change (i.e., the average frequency) is related to the average axial velocity of the scatterers in the velocity sample volume via the Doppler equation (Eq. (2.9)). Thus, assessment of average particle velocity is reduced to estimation of average Doppler signal frequency. The main aspects associated with PW Doppler blood velocity assessment are summarized below:

- range/velocity limitation

The phase detector can only detect phase differences unambiguously within the interval $(-\pi,+\pi)$, so that the maximum Doppler frequency equals $\pi \cdot P R F /(2 \pi)=$ $P R F / 2\left[\mathrm{~s}^{-1}\right]$. Substitution in the Doppler equation gives:

$$
\mathrm{v}_{\max }=\frac{\mathrm{c}_{\mathrm{us}} \cdot \mathrm{PRF}}{4 \mathrm{f}_{\mathrm{c}}}=\frac{\lambda \cdot \mathrm{PRF}}{4}
$$

or with Eq. (2.17):

$$
v_{\max }=\frac{c_{u s}^{2}}{8 f_{c} \cdot d_{\max }}
$$


where $d_{\max }$ is the maximum depth of investigation. Apparently, an inverse relationship exists between the depth of investigation and the maximum velocity detectable.

- transit time effect

Figure 2.8-a shows the real part of the Doppler signal as produced by the single particle of figure 2.5. The settings used are: rf pulse duration $\tau_{p}=3 / f_{c}$, rf pulse envelope is rectangular at a level of 10 arbitrary units, $f_{s}=4 f_{c}$, gate weighting is uniform with $G W=12$ sample points, package length $P L=64$ observations, and the phase shift of the rf segment in the gate over consecutive recordings equals $\pi / 2$. Obviously, the envelope of the Doppler signal reflects the sensitivity function of the velocity sample volume along the path followed by the particle. The amplitude spectrum of the Doppler signal, drawn in figure 2.8-b, indicates that the mean frequency approximates $0.25 P R F$, corresponding to a particle velocity of $0.125 P R F \cdot c_{u s} / f_{c}$ towards the transducer. Note that, although a single particle has been observed with a constant velocity, the Doppler signal has a nonzero bandwidth. This effect is known as the transit time effect, and is related to the finite presence of the particle in the velocity sample volume (Hoeks et al., 1991; Wilson, 1991). Since the Doppler signal is only nonzero for the time the particle requires to cross the sample volume, a spectral broadening is induced. If the effective length of the sample volume is measured as $S V_{\text {eff }}=c_{u s} \cdot \tau_{p} / 2$, then the effective transit time equals $\tau_{\text {trans }}=S V_{\text {eff }} / v$. Application of Eq. (2.9) under assumption of the uncertainty relations $\Delta f_{d} \cdot \tau_{\text {trans }}=1$ and $\Delta f_{c} \cdot \tau_{p}=1$, leads to the relation:

$$
\frac{\Delta f_{d}}{\bar{f}_{d}}=\frac{\Delta f_{c}}{\bar{f}_{c}}
$$

Apparently, the fractional width of the Doppler spectrum is a constant and equals the fractional width of the ultrasound pulse emitted (Newhouse et al., 1976). This implies that the spectral broadening of the Doppler signal increases with increasing $v$, i.e., with decreasing transit time. To illustrate this behavior, figure 2.8-c shows the Doppler signal of a single particle moving at half the speed of the original one. The number of periods in the Doppler signal has remained the same as in 2.8a, but the transit time has doubled. As a result, the spectrum, now centered around 0.125 PRF (Fig. 2.8-d), has become narrower, while the peak value has increased. Moreover, the area below the spectrum has increased, which is due to the fact that the Doppler signal energy has doubled. Mark that when measuring blood velocity, the number of red blood cells in the sample volume remains fairly constant, 

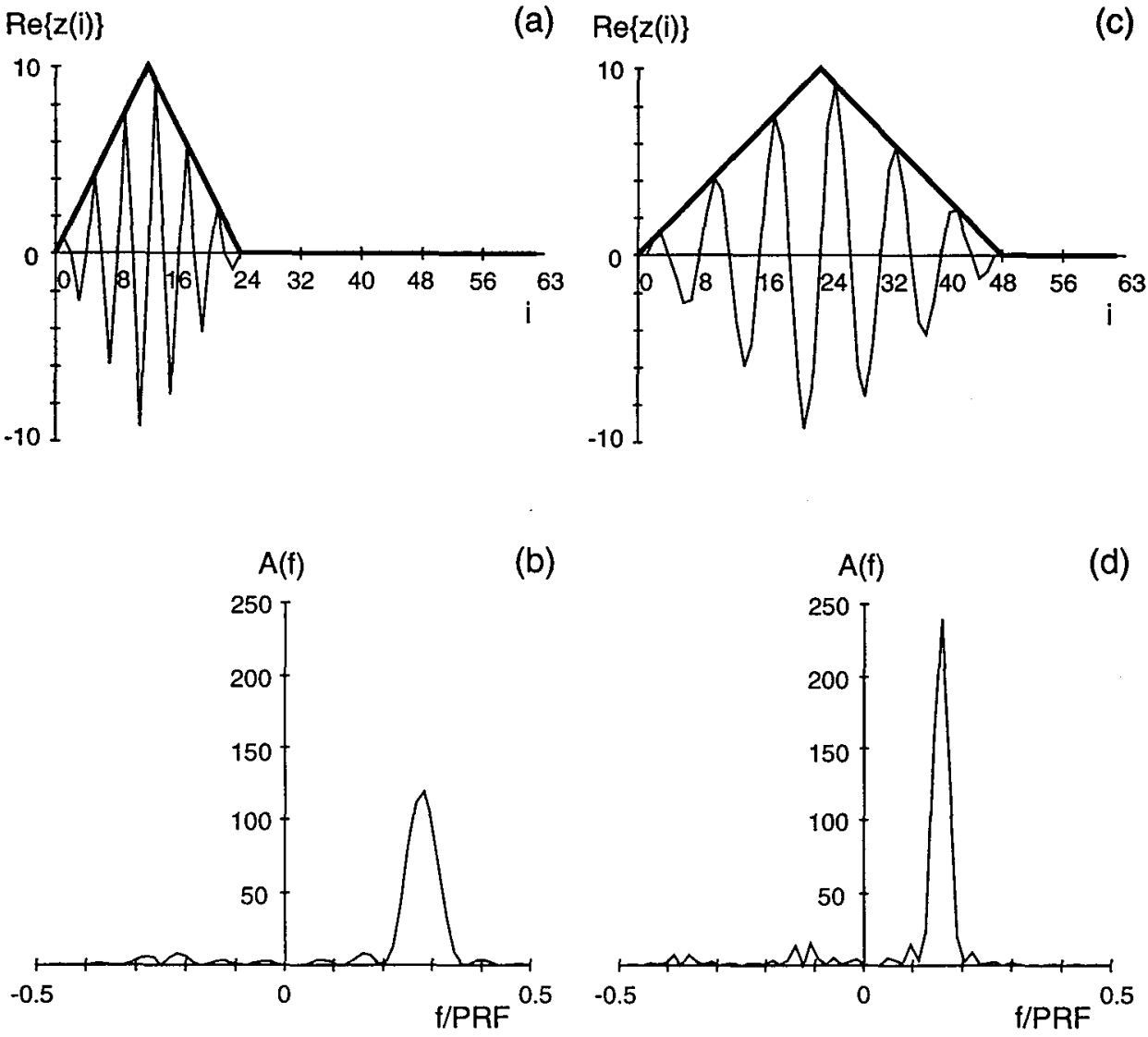

Figure 2.8. (a) Real part of the Doppler signal produced by the single particle of figure 2.5 (bold line: envelope). Settings used are: rf pulse duration $\tau_{p}=3 / f_{c}$, rf pulse envelope is rectangular at a level of 10 arbitrary units, $f_{s}=4 f_{c}$, uniform gate weighting with $G W=12$ sample points (conform figure 2.6), $P L=64$ observations, and the phase shift of the rf segment in the gate over consecutive recordings equals $\pi / 2$ (in accordance with figure 2.5). (b) Amplitude spectrum of the Doppler signal of (a). (c) Doppler signal of a particle moving at half the speed of the original one in (a). (d) Amplitude spectrum of the Doppler signal of (c). The nonzero bandwidths of the Doppler signals in (a) and (c) are due to the transit time effect.

independent of the average velocity. Consequently, the Doppler energy is independent of the blood velocity (neglecting effects as clustering and rouleaux formation). If blood flow is mainly transverse with respect to the ultrasound beam, the transit time effect is governed by the local beam width instead of the axial length of the velocity sample volume. 
- velocity resolution

The Doppler equation implies that the relative velocity uncertainty $\Delta v / v_{\max }$ equals the relative Doppler frequency uncertainty $\Delta f_{d} / f_{d, \max }$. Hence, for a given $f_{d, \max }$, velocity resolution is improved by decreasing $\Delta f_{d}$. A reduction of $\Delta f_{d}$ is achieved by elongation of the particle observation time, either being limited by the particle transit time or by the Doppler signal duration $P L \cdot \tau_{P R F}$, whichever is the smallest. The transit time can be increased by increasing the size of the velocity sample volume (by using longer ultrasound pulses, a proportionally larger gate and a wider beam), but this has the side effect that the velocity resolution in space decreases. On the other hand, enlargement of the package length is at the expense of the resolution of velocity in time, which can become particularly problematic when particle velocity is not stationary. Thus, whatever the settings of the Doppler system are, they always express a compromise between velocity range resolution, space resolution and time resolution.

- Doppler signal-to-noise ratio

Real rf signals are corrupted by white, additive noise, partly originating from the receiver amplifier (electronic noise) and partly introduced by the analog to digital converter (quantization noise). Writing the $\mathrm{rf}$ signal $r f\left(n \tau_{s}\right)$ as the sum of a (limited bandwidth) signal part $f_{s}\left(n \tau_{s}\right)$ and a white noise part $f_{N}\left(n \tau_{s}\right)$, the rf signal-to-noise ratio is defined as:

$$
\mathrm{SNR}_{\mathrm{rf}}=\frac{E\left\{\mathrm{rf}_{\mathrm{S}}{ }^{2}\right\}}{E\left\{\mathrm{rf}_{\mathrm{N}}{ }^{2}\right\}}
$$

where $E$ denotes statistical expectation. Eq. (2.23) may be rewritten as:

$$
z=\underbrace{\frac{2}{G W} \sum_{n \tau_{s} \in \text { gate }} f_{s}\left(n \tau_{s}\right) \cdot e^{-j \cdot 2 \pi f_{c} \cdot n \tau_{s}}}_{Z_{S}}+\underbrace{\frac{2}{G W} \sum_{n \tau_{s} \in \text { gate }}\left(n f_{s}\left(n \tau_{s}\right) \cdot e^{-j \cdot 2 \pi f_{c} \cdot n \tau_{s}}\right.}_{Z_{N}}
$$

so that the Doppler signal-to-noise ratio equals:

$$
S N R_{\text {Dop }}=\frac{E\left\{\left|z_{S}\right|^{2}\right\}}{E\left\{\left|z_{N}\right|^{2}\right\}}
$$


Mark that the Doppler component $z_{N}\left(i \cdot \tau_{P R F}\right)$ is a white noise signal, even if $r_{N}\left(n \tau_{s}\right)$ were band limited (e.g. due to band pass filtering of the rf signals). This is explained by the fact that no correlation exists between the rf noise components of the different observations in the package. The extent to which the noise in the rf signals eventually influences the velocity estimate is determined by the level of $S N R_{D o p}$ as well as the sensitivity of the mean Doppler frequency estimator to the respective component signals $z_{S}\left(i \cdot \tau_{P R F}\right)$ and $z_{N}\left(i \cdot \tau_{P R F}\right)$. As long as $G W \leq \tau_{p} \cdot f_{s}$, the phase of $f_{s}\left(n \tau_{s}\right)$ increases, in a rough approximation, linearly with $n \tau_{s}$ over the gate. At the same time $r_{N}\left(n \tau_{s}\right)$ exhibits a purely random behavior. Consequently, $r_{s}\left(n \tau_{s}\right)$ adds constructively in the calculation of the complex Doppler point $z$ while $\pi_{N}\left(n \tau_{s}\right)$ cancels out. According to Eq. (2.28), E\{|zs $\left.\left.\right|^{2}\right\}$ is independent of $G W$ whereas $E\left\{\left|z_{N}\right|^{2}\right\}$ decreases with increasing $G W$. Thus, $S N R_{D o p}$ can be improved by increasing the $\mathrm{rf}$ pulse duration and adapting the gate width accordingly ( $G W=$ $\tau_{p} \cdot f_{s}$ ). For gate widths exceeding the pulse duration, echoes from independent locations (with a mutual axial distance larger than $c_{u s} \cdot \tau_{p} / 2$ ) contribute incoherently to the rf signal segment in the gate. As a result, $E\left\{\left|z_{s}\right|^{2}\right\}$ starts to behave inversely proportional to $G W$, causing $S N R_{D o p}$ to become a constant, independent of $G W$. According to this reasoning, an optimal behavior in terms of axial resolution and Doppler signal-to-noise ratio is attained by letting the gate width equal the pulse duration.

It is noted that the phase detector incorporates a low pass filter that is applied to the demodulated rf signal. Equivalently, the Doppler signal-to-noise ratio can be improved by applying a band pass filter to the raw rf signals, carefully matched to the frequency content of $r_{s}\left(n \tau_{s}\right)$. This has the effect that $r_{N}\left(n \tau_{s}\right)$ becomes band limited with the same quality factor $Q$ as $f_{s}\left(n \tau_{s}\right)$ and with $E\left\{f_{N}^{2}\right\}$ reduced. Again, it follows that $S N R_{D o p}$ can be improved by increasing the rf pulse duration (and thus decreasing the signal bandwidth with respect to the noise bandwidth). However, this time, manipulation of $G W$ hardly influences $S N R_{D o p}$. This observation, supplemented with considerations concerning the validity of the assumed linear phase behavior of $r f_{S}\left(n \tau_{s}\right)$ over the gate, has led to the concept of subsample volume Doppler processing, yielding a reduced variance in the eventual velocity estimate and to be discussed in subsection 2.4.1.4.

\section{- mismatch of center frequency}

Velocity estimation based on Doppler processing only works out correctly if the frequency of the reference signal applied matches the mean frequency of the echo signals received. Due to the effect of frequency dependent attenuation, however, the mean frequency of the echo signals tends to decrease with distance, resulting in a 
significant bias in the velocity estimates. This effect can be minimized, at the expense of spatial resolution, by using narrow band ultrasound pulses.

\subsubsection{Tissue filter}

The Doppler signal originating from a velocity sample volume enclosing flowing blood, may be modeled as consisting of three distinct components, as shown in figure 2.9-a: a wide band noise signal $z_{N}\left(i \cdot \tau_{P R F}\right)$, a limited bandwidth signal associated with the local motion of red blood cells, and a narrow band signal reflecting tissue motion. The latter two signals both contribute to $z_{S}\left(i \cdot \tau_{P R F}\right)$. Thereby, the bandwidth of the blood component signal is governed by the transit time effect as well as the blood velocity dispersion in the velocity sample volume. Two separate mechanism can be identified, accounting for the presence of the tissue component in the Doppler signal. In the first place, for velocity sample volumes positioned close to the
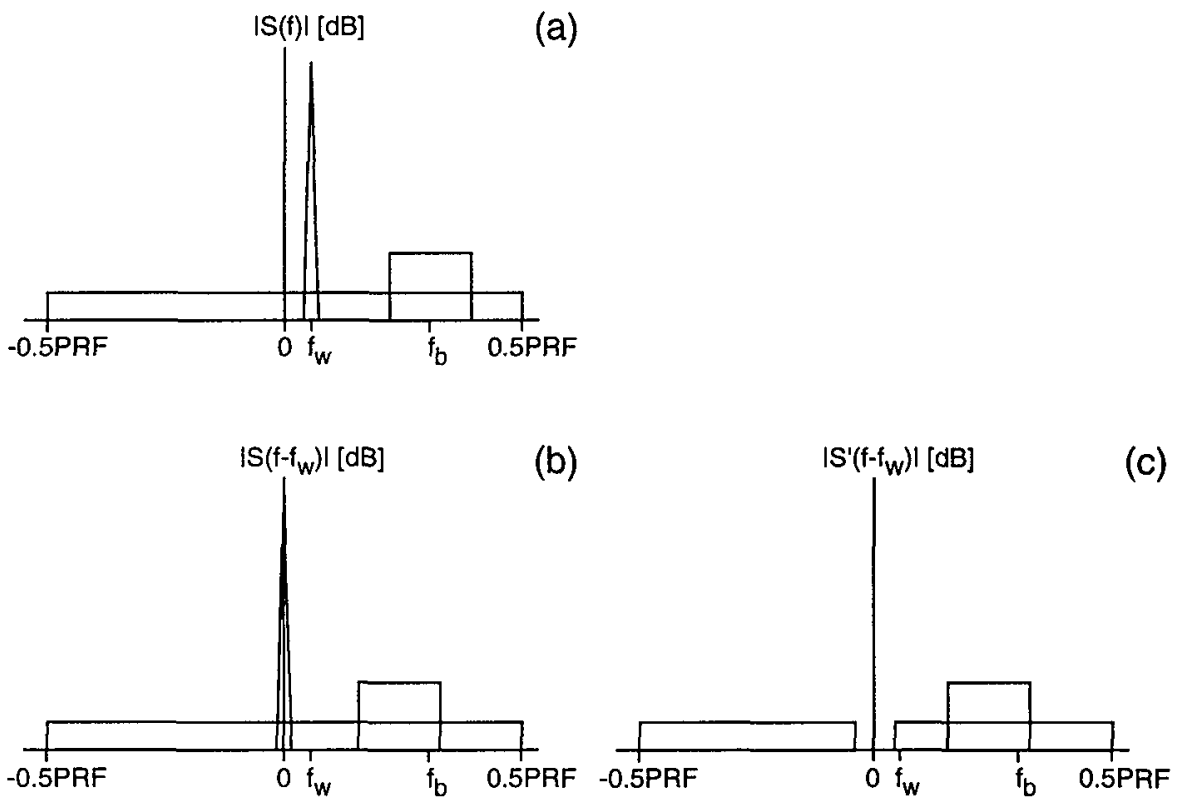

Figure 2.9. (a) Schematic representation of a typical clinical Doppler power spectrum. The Doppler signal consists of a wide band noise signal, a limited bandwidth signal associated with the motion of red blood cells, centered around an average Doppler frequency $f_{b}$, and a narrow band signal reflecting tissue motion (especially of the vessel wall), centered around $f_{w}$. (b) After detection of $f_{w}$, the combined Doppler spectrum can be shifted such, that the tissue peak becomes aligned around zero frequency. (c) The shifted tissue peak is effectively removed by a high pass filter, such as the regression filter. 
vessel wall, the tissue component results from the finite decay time of the strong wall echoes. In the second place, throughout the lumen, the tissue signal can be attributed to multiple reflection or reverberation: a mechanism in which echoes from intermediate tissue make a second round trip after having hit the transducer, to rearrive at the same instant as the signals from the velocity sample volume, this way falsely contributing to the signal in the gate. Since tissue motion is generally coherent (no velocity dispersion) and slow (giving limited transit time spectral broadening), the corresponding component in the Doppler signal has a narrow bandwidth and a mean frequency close to zero. Typically, the tissue signal amplitude is 20 to 40 $\mathrm{dB}$ larger than the blood signal amplitude.

Since the length $P L$ of the Doppler signal is very limited in color velocity imaging, Fourier analysis of the Doppler signal, giving a very poor spectral resolution, is not the most suitable method for extracting the mean blood frequency (apart from its computational complexity). Instead, various time domain algorithms have been proposed. Second-order autoregressive modeling enables the simultaneous estimation of both the positions of the vessel wall and blood induced peaks in the Doppler spectrum (Loupas and McDicken, 1990). However, computer evaluations have indicated that the combination of a tissue filter, rejecting the tissue signal, followed by a robust mean frequency estimatcr, is more fruitful (Brands and Hoeks, 1992). In its basic form, the tissue filter is an ordinary IIR or FIR high pass filter. For minimum affection of the blood component in the Doppler signal, the filter should have a low cut-off frequency and a sharp roll-off. However, these properties imply a long settling time, leaving only very few (if any) points in the filtered Doppler signal on which the mean blood frequency estimation can be based. A recent filter that does combine a zero settling time with second-order high pass properties is the regression filter (Hoeks et al., 1991), subtracting linear regression lines from both the real and imaginary parts of the Doppler signal. A refined version of this filter has been incorporated in the measurement setup of Chapter 6. The modification enables the filter to handle also tissue signals with relatively high Doppler frequencies as, e.g., induced by motion of the transducer. It involves the localization of the spectral tissue peak followed by appropriate adaptation of the rejection band of the regression filter. Since the tissue peak is very narrow, the tissue component signal may be modeled as consisting of a single complex harmonic signal with a constant amplitude and with an angular frequency $\omega_{t}$. An estimate of $\omega_{t}$ is obtained by minimizing the error function:

$$
\operatorname{err}\left(\hat{\omega}_{\mathrm{t}}\right)=\sum_{i=1}^{\mathrm{PL}-1}\left\{\Delta \phi(i)-\hat{\omega}_{\mathrm{i}} \cdot \tau_{\mathrm{PRF}}\right\}^{2}
$$


where $\Delta \phi(i)=\arg \left\{z\left(i \cdot \tau_{P R F}\right)\right\}-\arg \left\{z\left((i-1) \cdot \tau_{P R F}\right)\right\}$. It turns out that the solution to minimizing Eq. (2.30) is just the output of the instantaneous frequency detector (Van Leeuwen et al., 1986):

$$
\hat{\omega}_{t}=\frac{P R F}{P L-1} \sum_{i=1}^{P L-1} \arg \left\{z\left(i \cdot \tau_{P R F}\right) \cdot z^{*}\left((i-1) \cdot \tau_{P R F}\right)\right\}
$$

where $z^{*}$ is the complex conjugate of $z$. The regression filter is adapted to the tissue peak by multiplying the Doppler signal $z\left(i \cdot \tau_{P R F}\right)$ by the complex exponent $\operatorname{exp(-}$ $\left.j \cdot i \cdot \omega_{t} \cdot \tau_{P R F}\right)$. This causes the frequency spectrum of $z$ to be shifted over $-\omega_{t}$, so that the tissue peak becomes aligned around zero (Fig. 2.9-b). Subsequent application of the regression filter to the shifted Doppler signal effectively rejects the tissue signal, leaving a gap around zero frequency (Fig. 2.9-c). Then the mean blood signal frequency is approximately equal to the mean frequency of the filtered Doppler signal plus $\omega_{t}$. To avoid erroneous operation in the absence of a tissue signal, the regression filter is applied to the unshifted Doppler signal if the estimated value of $\left|\omega_{t}\right| \mathrm{ex}$ ceeds a predefined threshold (a small fraction of the full scale).

The consequence of the presence and rejection of a tissue component in the Doppler signal is, that the very lowest blood velocities cannot be measured. In particular the assessment of blood velocity close to the vessel wall is problematic.

\subsubsection{Doppler autocorrelator}

After application of the tissue filter, the mean Doppler frequency is estimated as well as some parameter values revealing whether the mean Doppler frequency actually reflects blood velocity. According to today's "gold standard" in color velocity imaging, the Doppler signal is fed to a complex autocorrelator (see Fig. 2.7), devermining the correlation coefficients $C_{\text {out }}(0)$, which equals the mean Doppler power, and $C_{\text {out }}\left(\tau_{P R F}\right)$, which is calculated as:

$$
\mathrm{C}_{\text {out }}\left(\tau_{P R F}\right)=\frac{1}{\mathrm{PL}-1} \sum_{\mathrm{i}=1}^{\mathrm{PL}-1}\left\{z\left(\mathrm{i} \cdot \tau_{\mathrm{PRF}}\right) \cdot \mathrm{z}^{*}\left((\mathrm{i}-1) \cdot \tau_{\mathrm{PRF}}\right)\right\}
$$

The two correlation coefficients are transferred to the block "combination and selection" in figure 2.7 for further processing. It has been proven by Kasai et al. (1985) that the mean angular Doppler frequency $\bar{\omega}_{d}=2 \pi \bar{f}_{d}$ is accurately approximated by: 


$$
\hat{\bar{\omega}}_{\mathrm{d}}=\mathrm{PRF} \cdot \arg \left\{\mathrm{C}_{\text {out }}\left(\tau_{\mathrm{PRF}}\right)\right\}
$$

Eq. (2.33) especially holds for Doppler signals with symmetric spectral distributions, as found if velocity sample volumes are small and the transit time effect is the major cause of spectral broadening. The popularity of the Doppler autocorrelation approach to estimating $\bar{\omega}_{d}$ is explained by its simplicity, robustness and insensitivity to the noise component signal $z_{N}$. Concerning robustness, the Doppler autocorrelation method is insensitive to aliasing in the sense that as long as $\left|\bar{f}_{d}\right| \leq P R F / 2$, the mean Doppler frequency is determined correctly, not being affected if part of the blood Doppler spectrum would exceed the valid frequency range as defined by Nyquist (Van Leeuwen et al., 1986). Concerning sensitivity to noise, according to Eq. (2.32), high amplitude Doppler points (with a good SNR) contribute stronger to $\hat{\bar{\omega}}_{d}$ than low amplitude points (with a poor SNR). In the presence of a stationary blood flow pattern, the variance in $\hat{\bar{\omega}}_{d}$ due to the noise signal $z_{N}$ can be reduced by increasing the number of points $P L$ over which is averaged.

\subsubsection{Recognition of false blood velocity estimates}

Doppler signals originating from velocity sample volumes that do not enclose flowing blood, lack a blood component signal. Consequently, after application of the tissue filter, only a wide band noise signal is left. The gap in the noise spectrum around zero frequency, induced by the tissue filter, causes the mean Doppler frequency estimated to drift towards plus or minus the full scale. On the other hand, strong tissue signals partly leaking through the tissue filter cause the mean Doppler frequency to drift towards zero frequency (whether the blood component is present or not). In both cases the velocity estimated is considered to be meaningless. According to the Doppler processing scheme of figure 2.7, four signal parameters are available to distinguish these false estimates from those actually reflecting blood velocity. The parameters include the powers of both the raw Doppler signal and the signal rejected by the tissue filter, respectively $C_{i n}(0)$ and $C_{r e j}(0)$, supplemented with the power of the filtered Doppler signal, $C_{\text {out }}(0)$, and the complex autocorrelation coefficient $C_{\text {out }}\left(\tau_{P R F}\right)$ of the filtered Doppler signal. The coefficients $C_{i n}(0), C_{\text {rej }}(0), C_{\text {out }}(0)$ and $C_{\text {out }}\left(\tau_{P R F}\right)$ are combined to produce a doublet or triplet of parameters, including the velocity estimate itself, on which the segmentation of false and true estimates is based.

A high value of $C_{i n}(0)$ indicates that the velocity sample volume encloses part of a highly reflecting structure, like a vessel wall. A value of the ratio $C_{r e j}(0) / C_{i n}(0)$ close to 1 suggests that only noise has past the tissue filter, while a value of $\hat{\bar{\omega}}_{d}$ close 
to zero indicates leakage of the tissue filter. In all these cases, the validity of the velocity estimated should be doubted. A useful parameter providing additional information in this respect is defined as:

$$
\mathrm{BW}_{\text {Dop }} \stackrel{\text { def }}{=} \sqrt{1-\frac{\left|\mathrm{C}_{\text {out }}\left(\tau_{\mathrm{PRF}}\right)\right|}{\mathrm{C}_{\text {out }}(0)}} \quad\left(0 \leq \mathrm{BW}_{\text {Dop }} \leq 1\right)
$$

It has been shown (Kasai et al., 1985) that $B W_{D o p}$ is proportional to the Doppler signal bandwidth (i.e., the standard deviation of the Doppler spectrum) relative to the $P R F$. For good Doppler signal-to-noise ratios, $B W_{D o p}$ is a measure of the bandwidth of the blood component signal only. In the absence of a blood component signal or in case the Doppler signal-to-noise ratio is poor, $B W_{D o p}$ shifts towards 1 . Most time, false velocity estimates can be correctly distinguished from real ones by placing a threshold on $B W_{\text {Dop. }}$. However, due to the stochastic character of the Doppler signal, some false recognitions cannot be avoided. Moreover, in the presence of a large velocity dispersion in the velocity sample volume due to a wild blood flow pattern, $B W_{\text {Dop }}$ may exceed the threshold while the mean velocity estimated is still correct. In the context of subsample volume Doppler processing (section 2.4.1.4), a simulation experiment will be described, indicating how effectively real and false velocity estimates can be distinguished by thresholding the $B W_{D o p}$ parameter.

\subsubsection{Subsample volume processing}

The Doppler processing scheme heavily relies on the assumption that the phase of $f_{s}\left(n \tau_{s}\right)$ increases linearly over the gate. Due to the random spatial distribution of the tiny scatterers in the velocity sample volume, however, the actual phase linearity vanishes with depth. This suggests, that an improved performance in terms of a reduced variance in the velocity estimate is attained by splitting the gate $\left(G W=\tau_{p} \cdot f_{s}\right)$ into a number of short segments (over each of which the linear phase behavior does hold), and applying to each segment the Doppler processing scheme separately, up to the block "combination and selection" of figure 2.7. This modified scheme has been referred to as subsample volume Doppler processing (Hoeks et al., 1994). The length of the segments or subgates is set to one period at the emission frequency, which is sufficient to suppress the higher harmonics introduced by the multiplication of the rf signal with the reference signal, conform Eq. (2.23). However, for an adequate suppression of the noise signal $\pi_{N}\left(n \tau_{s}\right)$, an additional band pass filter is needed, matched to the frequency content of $r_{s}\left(n \tau_{s}\right)$ and to be applied to the raw $\mathrm{rf}$ signals prior to subsample volume Doppler processing. The modified approach to velocity estimation yields for each subgate a quartet coefficients $\left\{C_{i n}(0), C_{r e j}(0)\right.$, 


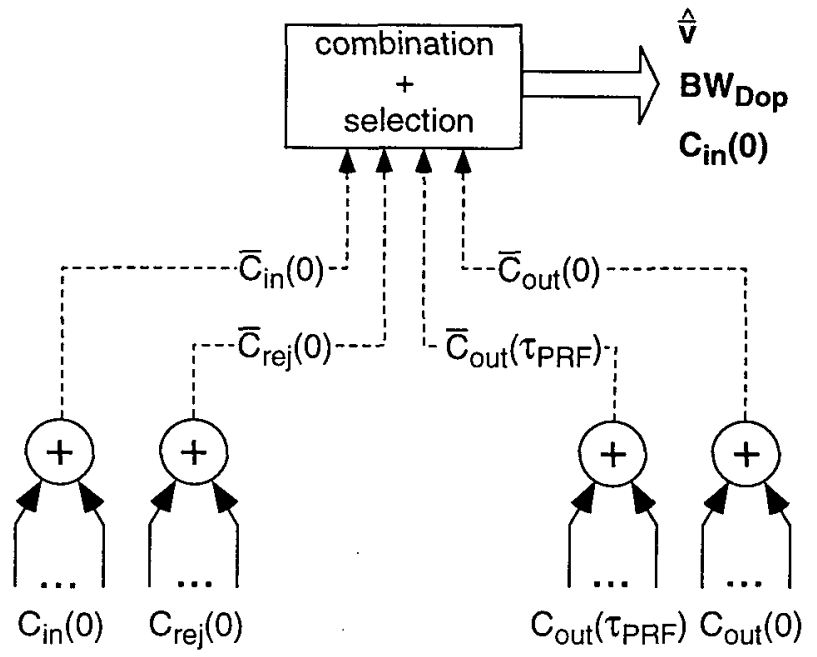

Figure 2.10. Subsample volume Doppler processing scheme. The gate has been split up into a number of subgates, each with a length of one period at the ultrasound emission frequency. Application of standard Doppler processing (Fig. 2.7) to the separate subgates yields for each subgate a quartet $\left\{C_{i n}(0), C_{\text {rej }}(0), C_{\text {out }}(0), C_{\text {out }}\left(\tau_{p r f}\right)\right\}$. These quartets are joined to produce average values, on which the final velocity and Doppler bandwidth estimations are based.

$\left.C_{\text {out }}(0), C_{\text {out }}\left(\tau_{P R F}\right)\right\}$. As depicted in figure 2.10 , all quartets are joined to produce average values, which are fed to the block "combination and selection" for calculation of the velocity (Eq. (2.33)) and Doppler bandwidth (Eq. (2.34)). It is pointed out that indeed the correlation coefficients are the parameters to average, and not the hypothetical subgate velocity and bandwidth estimates. This is because the latter procedure does not take into account the powers of the different (sub)Doppler signals, assigning equal weights to all subsample volumes, independent of local fluctuations in the Doppler signal-to-noise ratio.

A computer experiment was set up to investigate the characterization of velocity estimates on the basis of the $B W_{D o p}$ parameter under subsample volume Doppler processing. Using the simulation software described by Hoeks et al. (1993), various sets of independent packages of $\mathrm{rf}$ signal segments were generated. The $\mathrm{rf}$ signals all had a quality factor $Q$ of 4 (for the signal parts as well as the noise parts). The $\mathrm{rf}$ sample frequency equaled 4 times the central ultrasound emission frequency. The package length $P L$ equaled 8 , which is a fairly small value, but typical in color velocity systems. The gate, having a total width of 16 sample points (corresponding to the rf pulse duration), was subdivided into 4 equal subgates, so that each package yielded 4 subsample volume Doppler signals of 8 points. The mean Doppler 
(a)
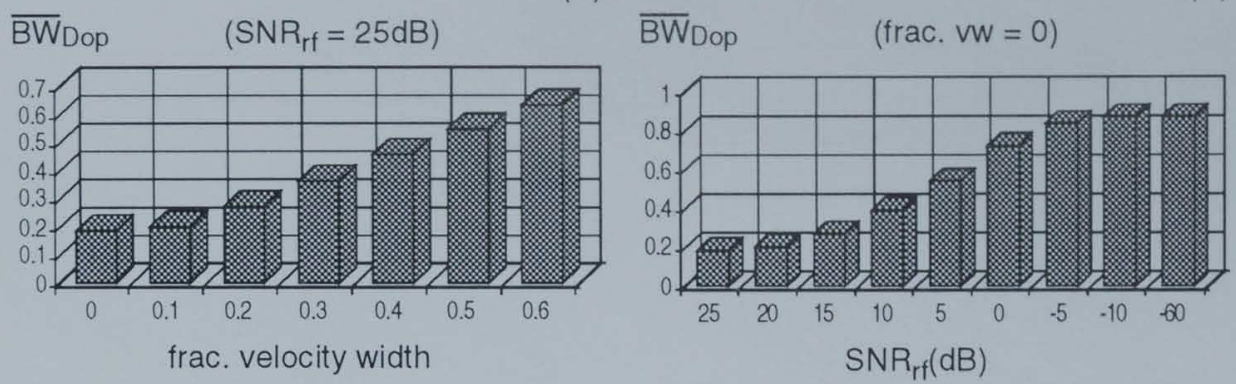

(c1)
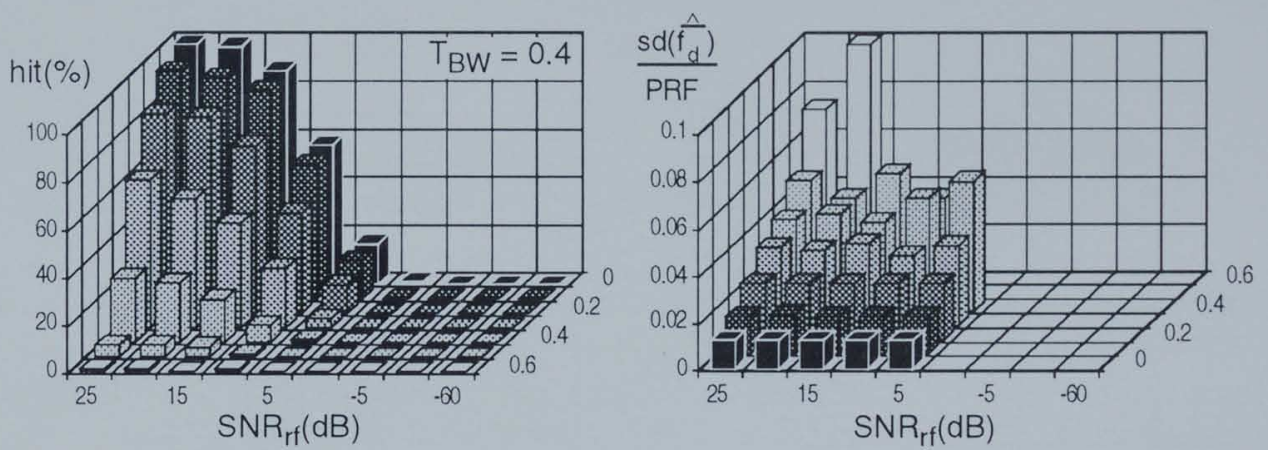

Figure 2.11. Evaluation of velocity estimation and characterization on the basis of subsample volume Doppler processing. For each combination of the rf signal-to-noise ratio $\left(S N R_{I_{f}}=25,20\right.$, $15,10,5,0,-5,-10,-60 \mathrm{~dB}$ ) and the relative axial velocity dispersion of particles in the velocity sample volume $\left(\Delta v / 2 v_{\max }=0.6,0.5,0.4,0.3,0.2,0.1,0\right)$, a set of 500 independent packages of $\mathrm{rf}$ signal segments was generated on a computer. The parameters that were constant over all sets are: $Q=4, f_{s}=4 f_{C}, P L=8, G W=16,4$ subgates per gate, $\bar{f}_{d}=0.2 P R F$. Each package of $\mathrm{rf}$ segments in a set yielded a single estimate of the mean Doppler frequency and a single estimate of the Doppler signal bandwidth. Each bar in the figure represents a result as averaged over a particular set. (a) Relation measured between the relative velocity dispersion and the average Doppler signal bandwidth $\overline{B W}_{D o p}$ for a good rf signal-to-noise ratio of $25 \mathrm{~dB}$. (b) Relation measured between the rf signal-to-noise ratio $S N R_{r}$ and the average Doppler signal bandwidth $\overline{B W}_{D o p}$ in the absence of velocity dispersion. (c) Left: for various combinations of the $\mathrm{rf}$ signal-to-noise ratio (horizontal axis) and the fractional velocity width (depth axis), the percentage is shown of velocity estimates that were recognized as actual blood velocity estimates. The recognition was based on thresholding the $B W_{D o p}$ parameter: If $B W_{D o p}$ exceeded a fixed threshold $T_{B W}$, the corresponding estimate was rejected. $T_{B W}$ ranged from 0.4 in (c1) to 0.7 in (c4). Right: the relative standard deviation of the mean Doppler frequency estimates as calculated over the accepted (hit) estimates within a set. Entries are empty if less than four estimates passed within one set. Note that the depth axis (frac. velocity width) is reversed as compared with the left figure. 
(c2)
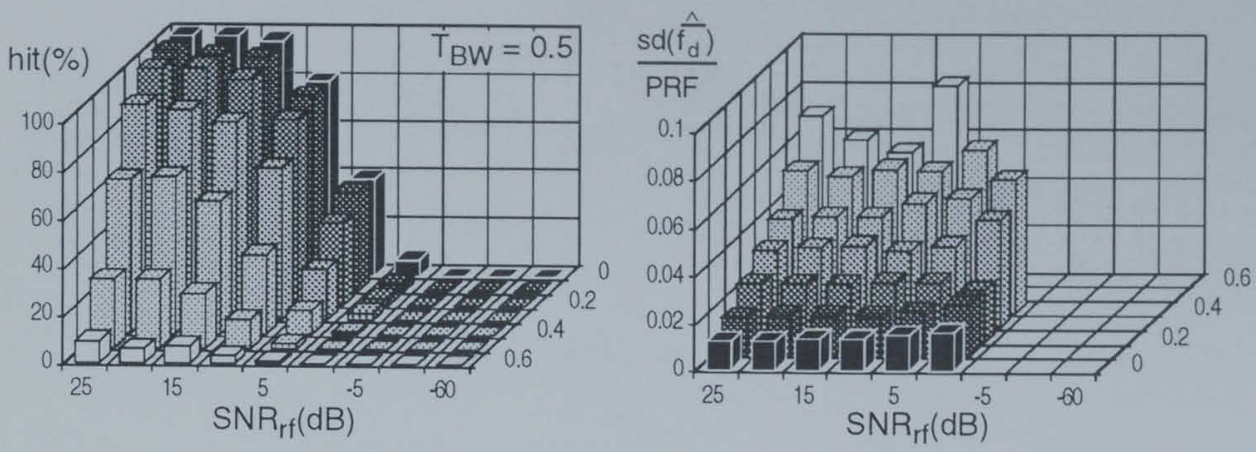

(c3)
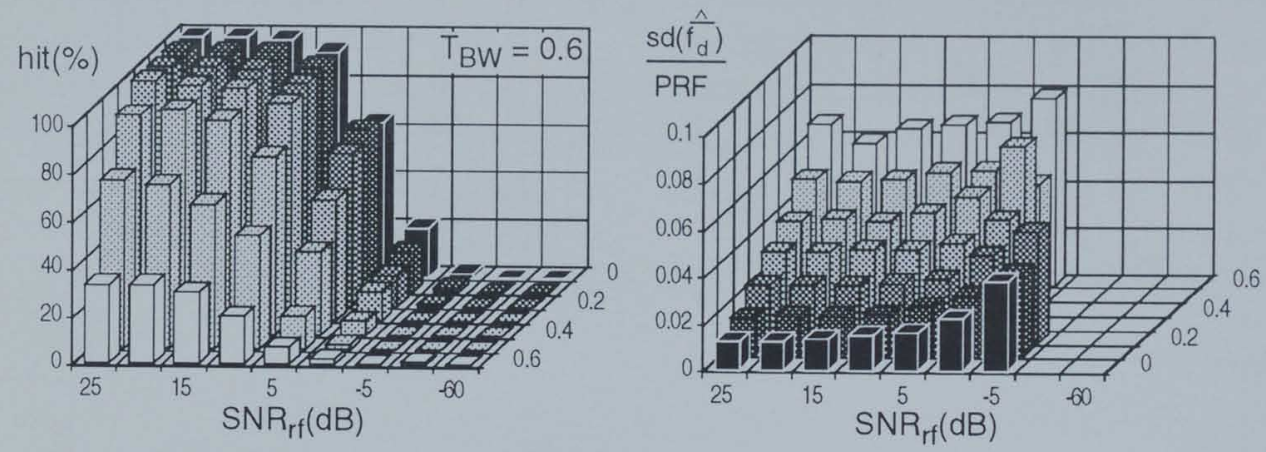

(c4)
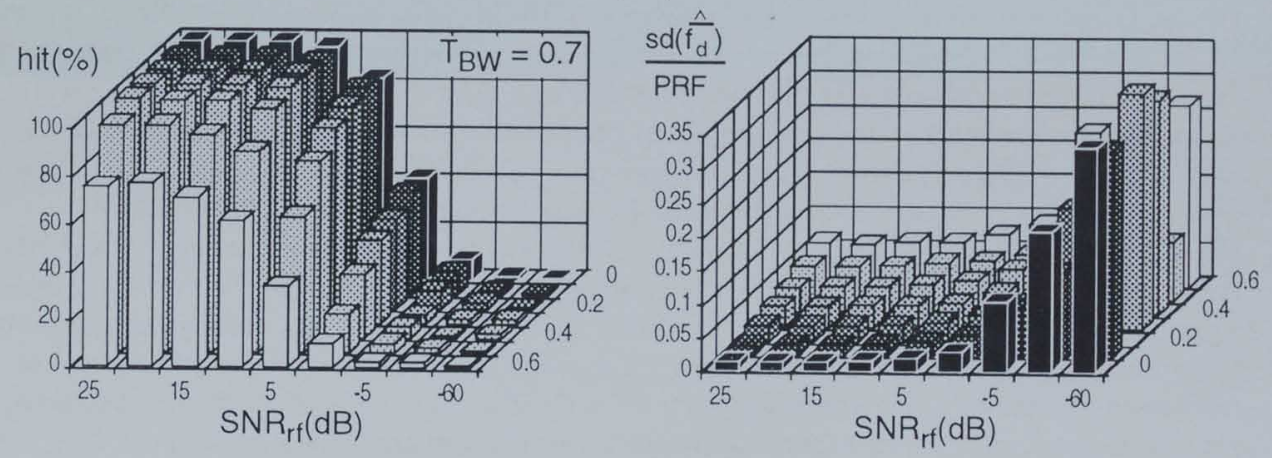

Figure 2.11. - continued 
frequency equaled 0.2 times the PRF. The parameters that were varied in the evaluation experiment included the $\mathrm{rf}$ signal-to-noise ratio, ranging from $-60 \mathrm{~dB}$ to $+25 \mathrm{~dB}$, and the velocity dispersion. The latter could be governed by building the $\mathrm{rf}$ signal segments in the gate up of 11 independent signals, each exhibiting a particular phase shift over consecutive observations. The velocity distributions this way generated were uniform, with widths ranging in equal steps from zero (no velocity dispersion) to 0.6 times the valid velocity range $\left(=2 v_{\text {max }}\right)$. For each combination of the two parameters $S N R_{r f}$ and fractional velocity width, the results, as shown in figure 2.11, are based on 500 independent packages of $\mathrm{rf}$ segments in the gate. Figure 2.11-a indicates that for a good $S N R_{r_{f}}$ of $25 \mathrm{~dB}$ an approximate linear relationship exists between $\overline{B W}_{D o p}$ and the fractional velocity width. In addition, figure 2.11 -b shows that $\overline{B W}_{D o p}$ also increases with decreasing $S N R_{H .}$. It is anticipated therefore, that the $B W_{D o p}$ parameter does not perfectly allow to distinguish between the situation in which a large velocity dispersion is present at a good $S N R_{r f}$ and the situation in which no blood component signals are present at all. This behavior is confirmed in figures 2.11-c1 to -c4. The left part of each figure shows, for various signal conditions, the percentage of velocity estimates that were recognized as reflecting actual blood velocity. The recognition was based on thresholding $B W_{D o p}$ : if $B W_{D o p}$ exceeded a predefined threshold $T_{B W}$, the corresponding velocity estimate was rejected. $T_{B W}$ ranged from 0.4 in figure $\mathrm{c} 1$ to 0.7 in figure c4. The right part of each figure shows the standard deviations of the mean Doppler frequencies, as averaged, for each signal condition, over the accepted (hit) estimates. In those cases where only four or less estimates passed, the $\operatorname{sd}\left(\bar{f}_{d}\right)^{\prime} \mathrm{s}$ were not calculated and the corresponding entries were left empty. Among the four thresholds applied, $T_{B W}=0.6$ seems to be the best compromise. Smaller thresholds lead to the rejection of relatively many correct velocity estimates (belonging to groups in which $\operatorname{sd}\left(\bar{f}_{d}\right) / P R F$ is of the order of 0.05$)$ while $T_{B W}=0.7$ allows false velocity estimates with a large $\operatorname{sd}\left(\bar{f}_{d}\right)$ to pass the test. Clearly, even for $T_{B W}=0.6$ misrecognitions cannot be avoided. As will be further discussed under color velocity imaging (section 2.5), additional postprocessing is required to compensate for these errors.

\subsubsection{Rf correlation interpolation}

A fundamental disadvantage of Doppler processing is, that the effect of frequency dependent attenuation may lead to a significant bias in the velocity estimated (last paragraph section 2.4.1). Recent rf cross correlation techniques avoid this problem, at the expense of computational cost, by omitting the reference signal. A method particularly attractive in the context of color velocity imaging, is the 


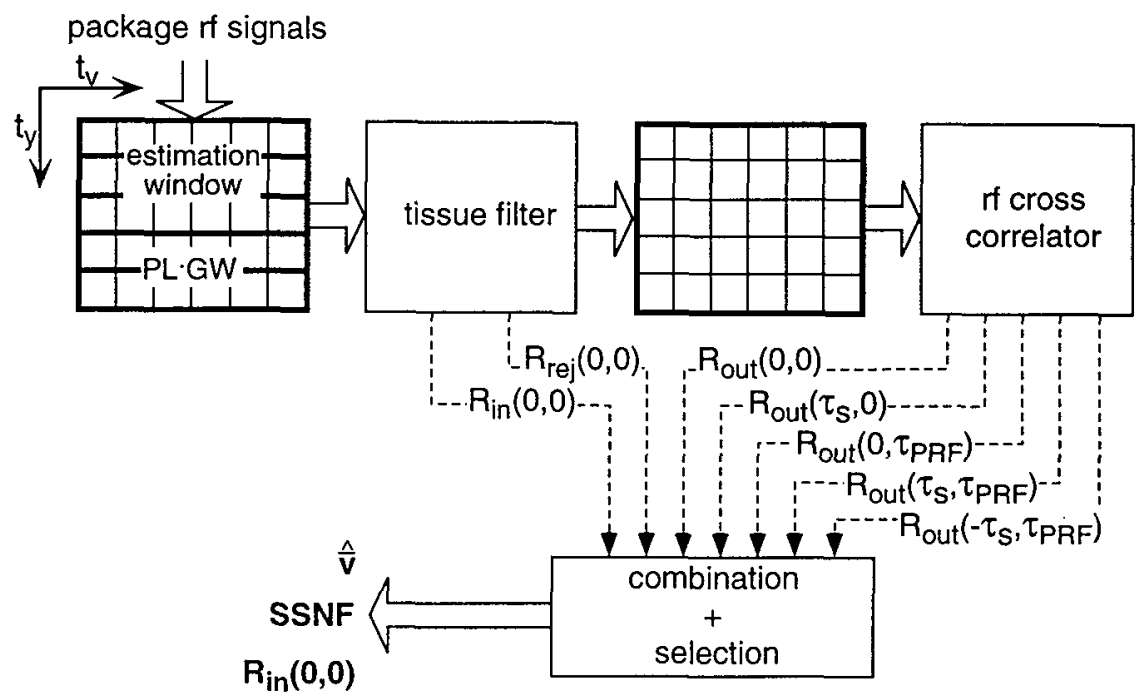

Figure 2.12. Rf correlation interpolation scheme. Velocity estimation is based on the same package of $P L$ consecutive rf signal segments of $G W$ sample points as is used in the Doppler processing scheme of figure 2.7. Stationary tissue components are rejected by a one-dimensional high pass filter that is applied separately to each of the GW signal distributions of $P L$ data points present in the estimation window. On the basis of 5 real $r f$ cross correlation coefficients, supplemented with the average raw rf signal power, $R_{i n}(0,0)$, and the average power of the tissue component rejected, $R_{r e j}(0,0)$, an average velocity $\hat{\bar{v}}$ is estimated as well as some parameter values that allow to determine whether $\hat{\bar{V}}$ actually reflects blood velocity, such as the signal-to(signal+noise) fraction of the filtered rf signal distribution, $S S N F_{r}$.

correlation interpolation algorithm, firstly described by De Jong et al. (1990) who employed it to measure myocardial tissue deformation. This method is based on the notion that a given spectral distribution of the rf signals leads to a known shape of the $\mathrm{rf}$ cross correlation function. This way, only five real cross correlation coefficients suffice to estimate mean particle velocity in the velocity sample volume, while a sampling frequency $f_{s}$ of the order of $4 f_{c}$ (Eq. (2.19)) is still sufficient. Writing the real cross correlation function as $R\left(\Delta t_{y}, \Delta t_{v}\right)$, where $\Delta t_{y}$ denotes time difference along the $\mathrm{rf}$ line (corresponding to difference in depth: $\Delta d=c_{u s} \cdot \Delta t_{y} / 2$ ) and $\Delta t_{v}$ denotes time difference between different rf signal recordings, the correlation coefficients needed include: $R(0,0), R\left(\tau_{s}, 0\right), R\left(0, \tau_{P R F}\right), R\left(\tau_{s}, \tau_{P R F}\right)$ and $R\left(-\tau_{s}\right.$, $\left.\tau_{P R F}\right)$. As has been shown by De Jong et al. (1990) for rectangular power spectra of both $r_{s}\left(n \tau_{s}\right)$ and $f_{N}\left(n \tau_{s}\right)$, and by Hoeks et al. (1993) for Gaussian spectra, the mean velocity $\left[\mathrm{m} \cdot \mathrm{s}^{-1}\right]$ is given by: 


$$
\hat{\bar{v}}=\frac{\mathrm{C}_{\mathrm{US}} \cdot \mathrm{PRF}}{2 \mathrm{f}_{\mathrm{s}}} \cdot \frac{\arctan 2\left[\mathrm{R}^{\prime}, \mathrm{R}\left(0, \tau_{\mathrm{PRF}}\right) \cdot \sin \left\{\arccos \left(\frac{\mathrm{R}\left(\tau_{\mathrm{s}}, 0\right)}{\mathrm{R}(0,0)}\right)\right\}\right]}{\arccos \left(\frac{\mathrm{R}\left(\tau_{\mathrm{s}}, 0\right)}{\mathrm{R}(0,0)}\right)}
$$

where $R^{\prime}=\left(R\left(\tau_{s}, \tau_{P R F}\right)-R\left(-\tau_{s}, \tau_{P R F}\right)\right) / 2$ and $\arctan 2$ denotes the arc tangent function with two arguments to retain phase information over all quadrants. The maximum velocity unambiguously detectable is $v_{\max }=0.25 \lambda \cdot P R F$, equaling the velocity limit encountered in Doppler processing (Eq. (2.24)). As indicated by figure 2.12, the correlation interpolation algorithm operates on the same data set of $P L \mathrm{rf}$ segments of GW points as the Doppler method of figure 2.7. Analog to Doppler processing, a tissue filter is applied to remove the stationary tissue component in the rf signals. Again, the tissue filter is a one-dimensional high pass filter, such as the linear regression filter, this time applied to the raw rf signals: the two-dimensional distribution of $P L \cdot G W \mathrm{rf}$ data points is handled as consisting of $G W$ signals of $P L$ points. In each of the GW signals the tissue contribution becomes manifest as a slow variation of the rf signal level, which can be effectively removed by the high pass filter. The remaining part of the two-dimensional rf signal distribution is fed to the $\mathrm{rf}$ cross correlator which calculates the five cross correlation coefficients. In the block "combination and selection" of figure 2.12, these coefficients are combined to produce an estimate of the mean particle velocity (Eq. (2.35)) and additional signal parameters. The central ultrasound emission frequency [s $^{-1}$ ] is estimated by (De Jong et al., 1990):

$$
\hat{f}_{c}=f_{s} \cdot \frac{\arccos \left(R\left(\tau_{s}, 0\right) / R(0,0)\right)}{2 \pi}
$$

and the rf signal-to-noise ratio is approximated by:

$$
\operatorname{SN} R_{r f}=\frac{R\left(0, \tau_{P R F}\right) / \cos \left(4 \pi \hat{\mathrm{f}}_{\mathrm{c}} \cdot \hat{\bar{v}} \cdot \tau_{\mathrm{PRF}} / \mathrm{c}_{\mathrm{uS}}\right)}{\mathrm{R}(0,0)-\mathrm{R}\left(0, \tau_{\mathrm{PRF}}\right) / \cos \left(4 \pi \hat{\mathrm{f}} \cdot \hat{\mathrm{v}} \cdot \hat{\bar{v}} \cdot \tau_{\mathrm{PRF}} / \mathrm{c}_{\mathrm{uS}}\right)}
$$

Like the $B W_{D o p}$ parameter in Doppler processing, $S \hat{N} R_{r f}$ may be used to distinguish between false velocity estimates (with poor signal-to-noise ratios) and true ones (with good signal-to-noise ratios). However, for good rf signal-to-noise ratios, the denominator in Eq. (2.37) takes a value close to zero and may even become negative, giving rise to large positive and negative outliers. A more robust parameter is the signal-to-(signal+noise) fraction: 
(a)
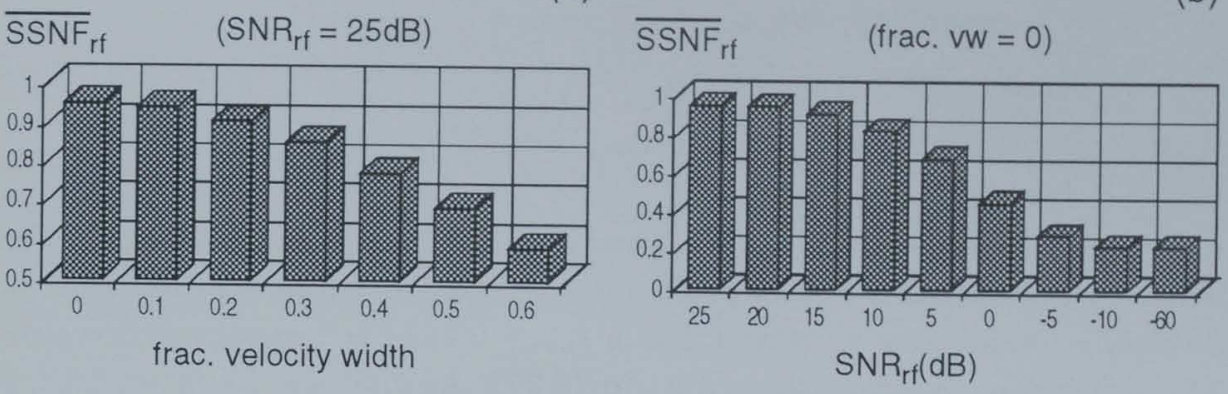

(c1)
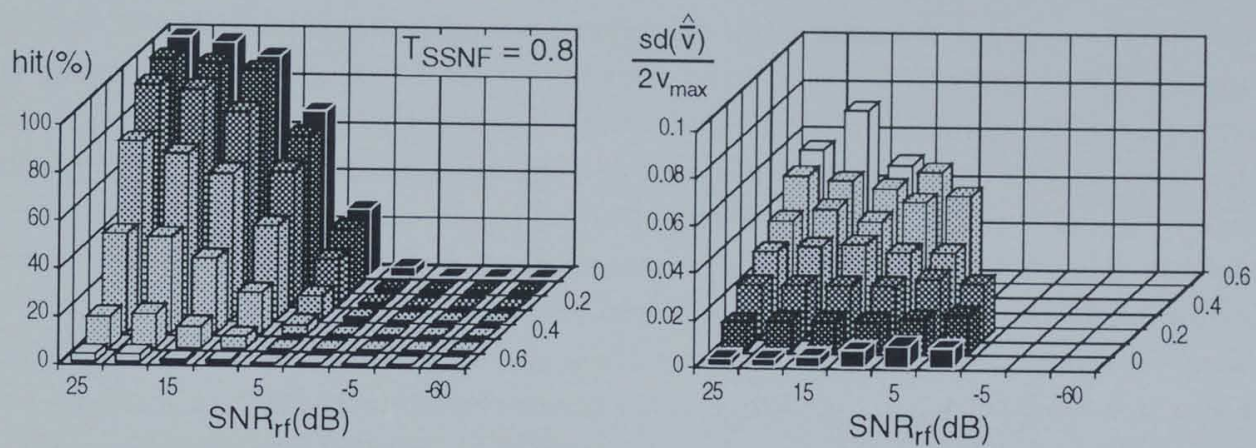

Figure 2.13. Evaluation of velocity estimation and characterization on the basis of rf correlation interpolation. For the same combinations as in figure 2.11 of the $\mathrm{rf}$ signal-to-noise ratio and the relative axial velocity dispersion of particles in the velocity sample volume, sets of 500 independent packages of $\mathrm{rf}$ signal segments were generated on a computer. The parameters that were constant over all sets are: $Q=4, f_{S}=4 f_{C}, P L=8, G W=16$, mean fractional velocity $\bar{v} / 2 v_{\max }$ $=0.2$. Each package of rf segments in a set yielded a single estimate of the mean fractional velocity and a single estimate of the rf signal-to-(signal+noise) fraction. Each bar in the figure represents a result as averaged over a particular set. (a) Relation measured between the relative velocity dispersion and the average $\mathrm{rf}$ signal-to-(signal+noise) fraction $\overline{S S N F}_{\mathrm{rf}}$ for a good $\mathrm{rf}$ signal-to-noise ratio of $25 \mathrm{~dB}$. (b) Relation measured between the rf signal-to-noise ratio $S N R_{n f}$ and the average rf signal-to-(signal+noise) fraction $\overline{S S N F}_{f f}$ in the absence of velocity dispersion. (c) Left: for various combinations of the rf signal-to-noise ratio (horizontal axis) and the fractional velocity width (depth axis), the percentage is shown of velocity estimates that were recognized as actual blood velocity estimates. The recognition was based on thresholding the SSNF r parameter: If SSNF $F_{\text {f }}$ did not exceed a fixed threshold $T_{S S N F}$, the corresponding estimate was rejected. TSSNF ranged from 0.8 in (c1) down to 0.5 in (c4). Right: the relative standard deviation of the velocity estimates as calculated over the accepted (hit) estimates within a set. Entries are empty if less than four estimates passed within one set. Note that the depth axis (frac. velocity width) is reversed as compared with the left figure. 

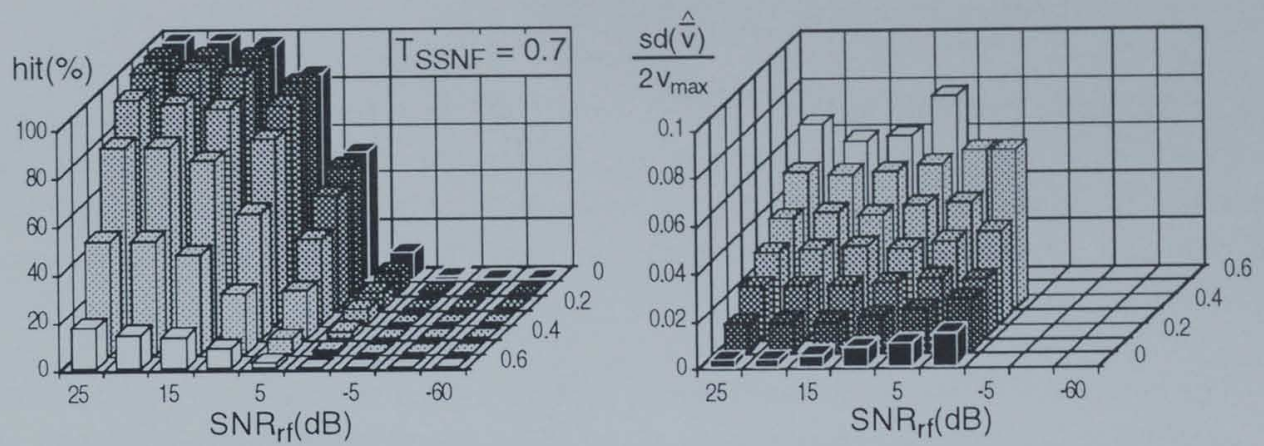

(c3)
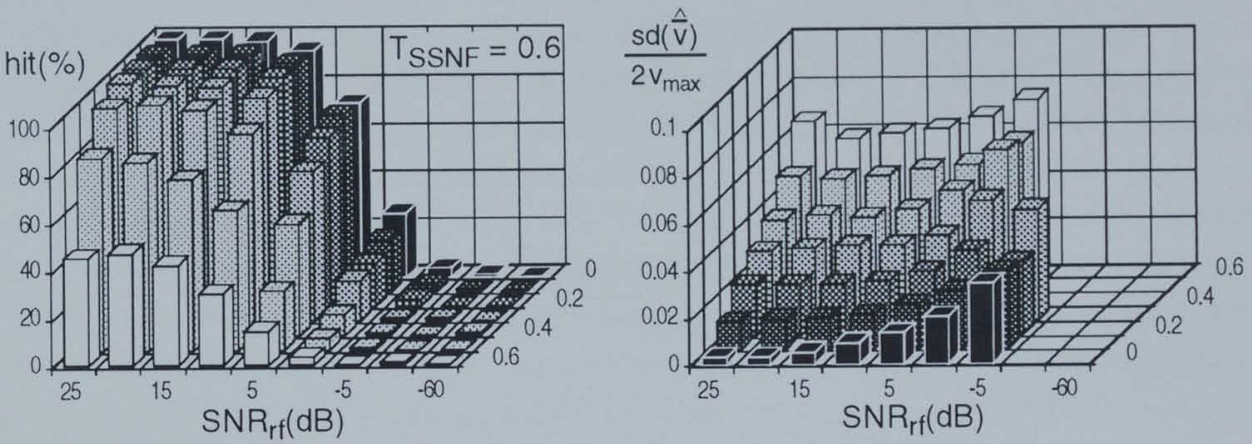

(c4)
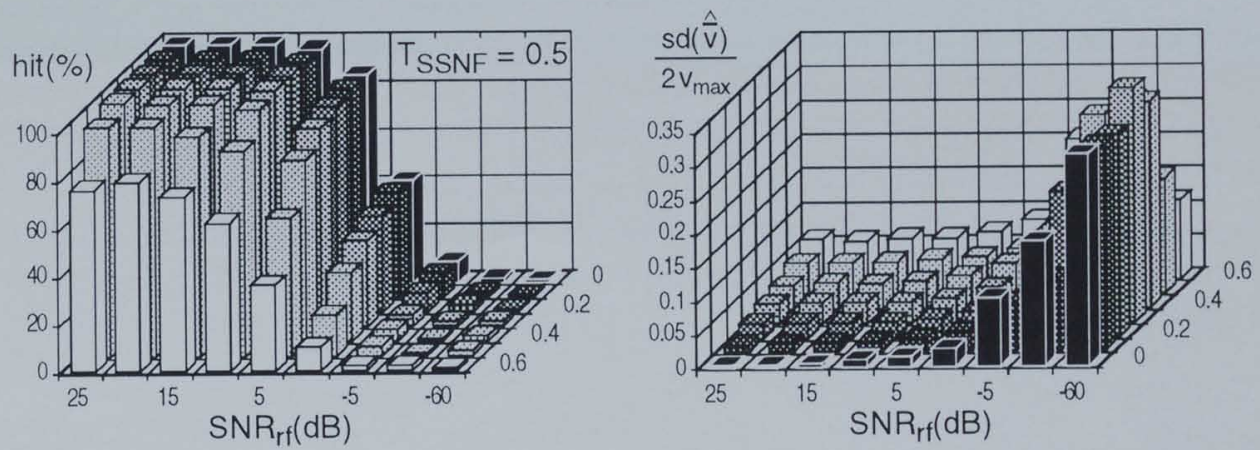

Figure 2.13. - continued 


$$
S S N F_{r f} \stackrel{\text { def }}{=} \frac{S \hat{N} R_{r f}}{S \hat{N} R_{r f}+1}=\frac{R\left(0, \tau_{P R F}\right)}{R(0,0) \cdot \cos \left(4 \pi \hat{f}_{c} \cdot \hat{v} \cdot \tau_{P R F} / c_{u s}\right)}
$$

The $S S N F_{r}$ is an estimate of the signal power $E\left\{r_{S}^{2}\right\}$ as a fraction of the total power $E\left\{r f_{S}^{2}\right\}+E\left\{f_{N}^{2}\right\}$ and ranges between 0 and 1. Analog to the Doppler processing scheme, the SSNF if parameter may be supplemented with the mean total powers of the raw and rejected rf signal distributions to give a complete characterization of the velocity estimate.

The computer experiment described in section 2.4.1.4 to investigate the identification of velocity estimates on the basis of the $B W_{D o p}$ parameter was repeated for the $S S N F_{n f}$ parameter in the context of rf correlation interpolation processing. The results are presented in figure 2.13. Figures 2.13-a and -b show that the $S S N F_{r f}$ is not solely a function of the $\mathrm{rf}$ signal-to-noise ratio but also of the fractional velocity width. As a result, a low velocity dispersion at a moderate $S N R_{r f}(5 \mathrm{~dB})$ cannot be distinguished perfectly from a large velocity dispersion at a very good $\mathrm{rf}$ signal-tonoise ratio $(25 \mathrm{~dB})$. This behavior is much like the behavior exhibited by the $B W_{D o p}$ parameter in section 2.4.1.4. The result of placing a threshold on $S S N F_{r f}$ is shown in figures 2.13-c1 to -c4. As in figure 2.11, the left part of each figure shows, for various signal conditions, the percentage of velocity estimates that were recognized as reflecting actual blood velocity. Velocity estimates were accepted if $S S N F_{r f}$ exceeded a predefined threshold $T_{S S N F}$, ranging from 0.8 in figure $\mathrm{c} 1$ down to 0.5 in figure $\mathrm{c} 4$. The right part of each figure shows the standard deviations of the velocity estimates relative to the valid velocity range $\left(=2 v_{\max }\right)$, as averaged, for each signal condition, over the accepted (hit) estimates. Obviously, the performance is comparable to the performance of the subsample volume Doppler processing method: by setting the threshold to a suitable value (as in figure 2.13 -c4, where $T_{S S N F}=0.6$ ) most velocity estimates, though not all, can be identified correctly. Thereby, the two methods produce comparable standard deviations of the hit velocity estimates. In the context of real-time color velocity imaging, subsample volume Doppler processing may be preferred because of its simplicity.

\subsection{Color velocity imaging}

In ultrasonic color velocity imaging, B-mode echo imaging is combined with local blood velocity assessment to produce, in real-time, a gray level structure image on which is superimposed, if present, a two-dimensional blood velocity distribution, encoded in color. Figure 2.14 presents the block scheme of an advanced color 


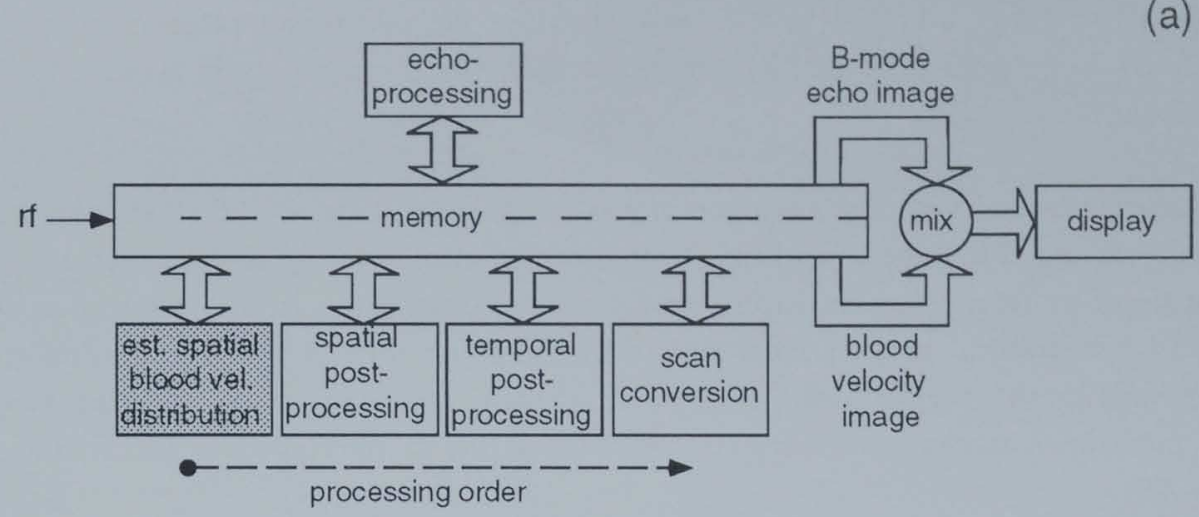

(b)

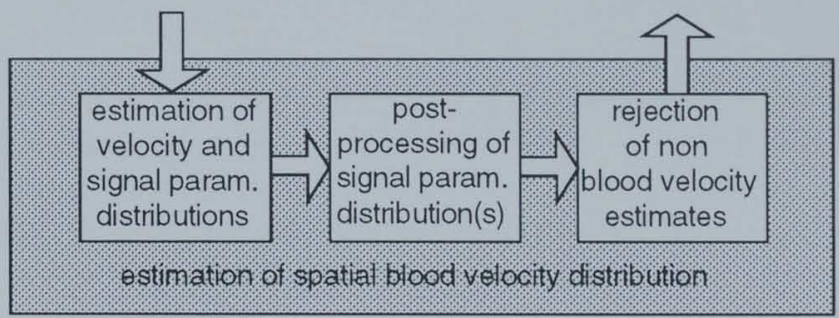

Figure 2.14. (a) Block scheme of an advanced color velocity imaging device, with exclusion of the data acquisition section and the control section. The rf signals acquired are stored in a digital memory that is accessible to both the B-mode echo image processing line, summarized by a single block (upper part) and the blood velocity image processing line (lower part, with the dashed arrow indicating the order of signal processing). The B-mode echo image and the blood velocity image are joined to produce a single image in which anatomical information is encoded in gray levels and blood velocity information in color. (b) The shaded block estimation of a spatial blood velocity distribution in (a) can be split up into three subblocks that represent the classification of velocity estimates on the basis of signal parameter values, and the subsequent rejection of non blood velocity estimates.

velocity mapper. The diagram is recognized as an extension of the B-mode echo scheme of figure 2.2. Apart from the echo processing line, which is now summarized by a single block, a second line is present, describing the assessment and postprocessing of a spatial blood velocity distribution out of an $\mathrm{rf}$ data frame. The central memory block has partly been subdivided into two sections, to indicate that the processing along the echo and velocity paths is separate, although the raw rf data may be shared. The data acquisition part has not been redrawn. As compared with the pure $\mathrm{B}$-mode echo imager, the $\mathrm{ADC}$ has an increased dynamic 
range ( $12+1$ bits), adapted to the, now relevant, signals backscattered from blood, which are very weak ( $-50 \mathrm{~dB})$ as compared with the strong signals reflected by the vessel walls.

As discussed, velocity measurements require a number of rf signals to be recorded along the same scan line. A one-dimensional velocity distribution in depth is obtained by employing multiple, partly overlapping gates, and a two-dimensional velocity distribution may be constructed in the same way as the B-mode image, by interrogating the target along a number of separate scan lines. Because at each scan position a package of consecutive $\mathrm{rf}$ lines has to be recorded, the frame rate drops to:

$$
\mathrm{FR}=\frac{\mathrm{PRF}}{\mathrm{n} \ln s \cdot \mathrm{PL}}
$$

where $P L$ is the package length and $n / n s$ is the number of scan positions. Eq. (2.39) assumes that one of the rf signals in each package is not only used in the velocity estimation, but also in the construction of the B-mode echo image. A more sophisticated scan protocol prescribes the recording of a separate $\mathrm{rf}$ signal at each scan position for the echo image. This has the advantage that the rf pulse length can be adapted to the specific requirements echo and velocity imaging each pose. The disadvantage is that the FR even further decreases.

\subsubsection{Postprocessing of velocity and parameter distributions}

The simulation experiments described in sections 2.4.1.4 and 2.4.2 indicate that the $B W_{D o p}$ parameter (for Doppler processing) or the $S S N F_{r}$ parameter (for rf correlation interpolation) can be used to determine whether flowing blood is present in the velocity sample volume, although the occurrence of incidental misrecognitions is inevitable. In color velocity imaging, the erroneous rejection of actual blood velocity estimates and acceptation of false estimates leads to a color velocity map with small holes inside blood velocity profiles and with noise spots outside. These mistakes can be effectively corrected by median filtering the spatial $B W_{D o p}$ or $S S N F_{r f}$ distribution, as is illustrated in figure 2.15. Figure 2.15-a shows a spatial $B W_{D o p}$ distribution, encoded in gray levels, as calculated on the basis of a single frame of rf signals. Light gray corresponds to a $B W_{D o p}$ level close to 1 whereas dark gray indicates a $B W_{D o p}$ level close to 0 . Each rectangular pixel corresponds to a velocity sample volume. The total image is build up of 32 sample volumes in lateral direction (corresponding to 32 linear array scan lines) by 125 sample volumes in axial direction. Other system settings used are: $f_{c}=6.1 \mathrm{MHz}, Q=4, f_{s}=4 f_{c}, P R F=$ 


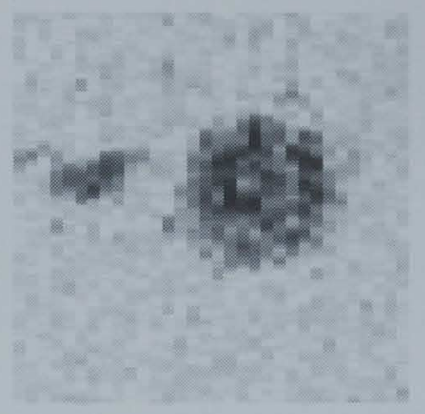

(a)

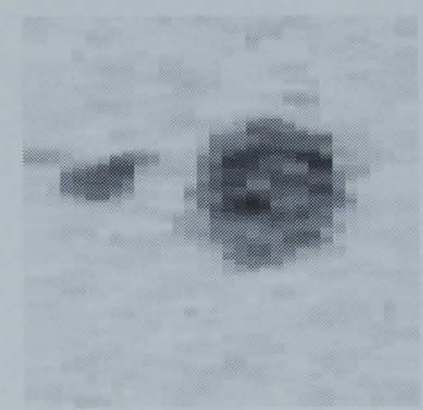

(c)

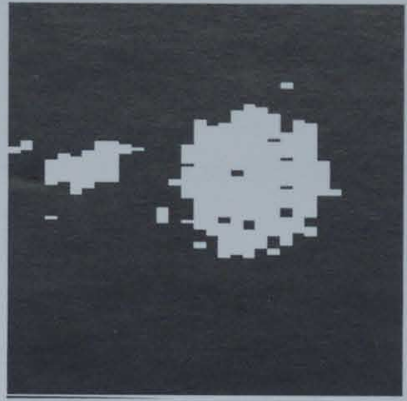

(b)

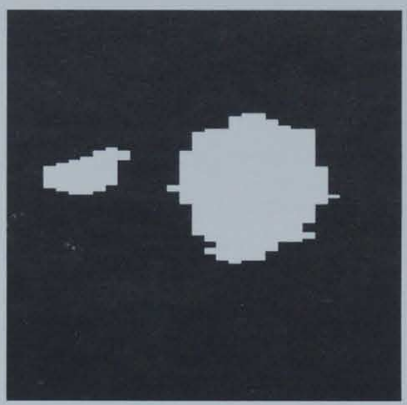

(d)

Figure 2.15. (a) Spatial $B W_{D o p}$ distribution as calculated on the basis of a single frame of $\mathrm{rf}$ signals. Light gray: $B W_{D o p}$ level close to 1 , dark gray: $B W_{D o p}$ level close to 0 . The region interrogated measured $2 \times 2 \mathrm{~cm}^{2}$ and included a carotid artery and a vein, both in cross sectional view. The lumens of the two vessels appear as dark regions in the BW Dop image (large region on the right: artery, small region on the left: vein). Each rectangular pixel corresponds to a velocity sample volume. The total image is build up of 32 sample volumes in lateral direction (corresponding to 32 linear array scan lines) by 125 sample volumes in axial direction. (b) Binary image obtained by applying a threshold $T_{B W}=0.6$ to the $B W_{D o p}$ distribution of (a). (c) Result of applying a $3 \times 3$ points median filter to the $B W_{D o p}$ distribution of (a).(d) Binary image obtained by applying a threshold $T_{B W}=0.6$ to image (c).

$8 \mathrm{kHz}, P L=15, G W=16$, subsample volume Doppler processing (each gate is split up into 4 subgates, adjacent gates share 3 subgates), rejection of stationary tissue components in the Doppler signals by the regression filter. The region interrogated measured $2 \times 2 \mathrm{~cm}^{2}$ and included a carotid artery and a vein, both in cross sectional view. The recording was taken during the systolic phase of the hart cycle. The lumens of the two vessels appear as dark regions in the $B W_{D o p}$ image (large region on the right: artery, small region on the left: vein). Application of a threshold $T_{B W}=$ 0.6 yielded the binary image of figure 2.15-b. According to the Doppler processing 
scheme, the velocity estimates of the white sample volumes are accepted and displayed in color in the color velocity image. The velocity estimates of the black sample volumes are rejected by setting them to zero. The isolated spots and holes are removed by a $3 \times 3$ points median filter applied to the $B W_{D o p}$ distribution (see Figs. 2.15-c and $-\mathrm{d}$ ).

The estimation of a spatial velocity distribution and a binary acceptance/rejection mask, such as the one of figure 2.15-d, is included in the scheme of figure 2.14 in the shaded block "estimation of spatial blood velocity distribution". The two-dimensional blood velocity distribution that is the output of this block, is further processed by the block "spatial postprocessing", which improves the spatial consistency of the blood velocity profile by application of a median or mean filter. However, the resulting distribution, though free from false estimates, is still not very suitable for presentation in a real-time sequence. Especially the irregular boundaries of the velocity profiles become unacceptable when observed in a rapid succession of color velocity images. This problem is further introduced in Chapter 3 and the additional temporal postprocessing required is the main subject of Chapter 6.

\subsubsection{Display of color velocity images}

Prior to the unification of anatomical and blood velocity information into a single color velocity image, a scan converter is applied (section 2.3.2), transforming the velocity distribution from velocity sample volume base to pixel base. In figure 2.14, the scan converter is positioned at the end of the velocity processing chain. The order of postprocessing and scan conversion, however, may be reversed, as will be discussed in Chapter 6.

It is assumed that the velocities in the velocity image are scaled with respect to the valid velocity range $\left(=2 v_{\text {max }}\right)$, so that pixel values range from -0.5 for maximum negative velocity (away from the transducer) to +0.5 for maximum positive velocity (towards the transducer). The structural echo image and the velocity image are joined such, that all velocity pixels with absolute values exceeding a threshold $T_{d}$ are displayed. The remaining areas are filled with echo pixels. A practical value of $T_{d}$ is the cutoff frequency of the tissue filter as a fraction of the PRF.

Whereas the structural information is encoded in gray levels (section 2.3.3), velocity information is displayed in color. Usually, red and blue gradations are used to represent positive and negative velocities, respectively (Evans, 1993; Eyer et al., 1981; Takeuchi, 1987). The color look-up table is designed on the basis of a lightness, hue 
and saturation color model (Levkowitz and Herman, 1993). One or more of the three color parameters are varied as a function of velocity. A common approach is to let the hue of the color change from red to orange to yellow with increasing positive velocity and from blue to cyan with increasing negative velocity. An additional increase of color lightness with a simultaneous decrease of color saturation yields a color bar with white tails at both the high velocity ends, this way diminishing the adverse effect of aliasing (whereby positive velocities exceeding $v_{\max }$ are mapped onto the negative end of the velocity scale and vice versa). Another option is, to vary red and blue lightness to encode mean velocity, and to change color hue (by mixing a variable amount of green) to provide an indication of velocity dispersion (as estimated by the $B W_{D o p}$ parameter). Then blood flow turbulence becomes manifest by red colors shifting towards yellow and blue colors drifting towards cyan.

\section{REFERENCES}

Bamber J.C. and Tristam M. (1988): Diagnostic ultrasound, in The physics of medical imaging, Webb S., Ed., Adam Hilger, Bristol, pp. 319-388.

Berkhoff A.P., Huisman H.J., Thijssen J.M., Jacobs E.M.G.P. and Homan R.J.F. (1994): Fast scan conversion algorithms for displaying ultrasound sector images, Ultrasonic Imaging 16, 87-108.

Bom N., Brommersma P.D. and Lancée C.T. (1993): Probes as used in cardiology with emphasis on transesophageal and intravascular applications, in Advances in ultrasound techniques and instrumentation, Wells P.N.T., Ed., Churchill Livingstone, New York, pp. 19-33.

Brands P.J. and Hoeks A.P.G. (1992): A comparison method for mean frequency estimators for Doppler ultrasound, Ultrasonic Imaging 14, 367-386.

Burns P.N. (1987): The physical principles of Doppler and spectral analysis, J. Clin. Ultrasound 15, 567-590.

Cloostermans M.J.T.M. and Thijssen J.M. (1983): A beam corrected estimation of the frequency dependent attenuation of biological tissues from backscattered ultrasound, Ultrasonic Imaging 5, 136147.

De Jong P.G.M., Arts T., Hoeks A.P.G. and Reneman R.S. (1990): Determination of tissue motion velocity by correlation interpolation of pulsed ultrasonic echo signals, Ultrasonic Imaging 12, 84-98.

Evans D.H. (1993): Techniques for color-flow imaging, in Advances in ultrasound techniques and instrumentation, Wells P.N.T., Ed., Churchill Livingstone, New York, pp. 87-107.

Evans D.H., McDicken W.N., Skidmore R. and Woodcock J.P. (1989): Doppler Ultrasound (John Wiley \& Sons, Chichester). 
Eyer M.K., Brandestini M.A., Phillips D.J. and Baker D.W. (1981): Color digital echo/Doppler image presentation, Ultrasound Med. Biol. 7, 21-31.

Ferrari L. and Jones J.P. (1985): The propagation of Gaussian modulated pulses in dissipative and/or dispersive media such as tissue, Ultrasound Med. Biology 11, 299-305.

Foster F.S. and Hunt J.W. (1978): The design and characterization of short pulse ultrasound transducers, Ultrasonics $16,116-122$.

Foster F.S. and Hunt J.W. (1979): Transmission of ultrasound beams through human tissue - focussing and attenuation studies, Ultrasound Med. Biol. 5, 257-268.

Foster S.G., Embree P.M. and O'Brien Jr. W.D. (1990): Flow velocity profile via time-domain correlation: error analysis and computer simulation, IEEE Trans. Ultrason. Ferro. Freq. Control 37, 164-175.

Gabor D. (1945): Theory of communication, J. IEE 93, 429-457.

Gere J.M. and Timoshenko S.P. (1985): Mechanics of Materials (Wadsworth International, Sydney).

Goodman J.W. (1968): Introduction to Fourier Optics (McGraw-Hill, San Francisco).

Harris R.A., Follett D.H., Halliwell M. and Wells P.N.T. (1991): Ultimate limits in ultrasonic imaging resolution, Ultrasound Med. Biol. 17, 547-558.

Hein I.A., Chen J.T., Jenkins W.K. and O'Brien Jr. W.D. (1993); A real-time ultrasound timedomain correlation blood flow meter: Part 1 - theory and design, IEEE Trans. Ultrason. Ferro. Freq. Control 40, 768-775.

Hein I.A. and O'Brien Jr. W.D. (1993): Current time-domain methods for assessing tissue motion by analysis from reflected ultrasound echoes - a review, IEEE Trans. Ultrason. Ferro. Freq. Control 40, 84-102.

Hill C.R. (1986): The generation and structure of acoustic fields, in Physical principles of medical ultrasonics, Hill C.R., Ed., Ellis Horwood, Chichester, pp. 68-92.

Hoeks A.P.G., Arts T.G.J., Brands P.J. and Reneman R.S. (1993): Comparison of the performance of the rf cross correlation and Doppler autocorrelation technique to estimate the mean velocity of simulated ultrasound signals, Ultrasound Med. Biol. 19, 727-740.

Hoeks A.P.G., Brands P.J., Arts T.G.J. and Reneman R.S. (1994): Subsample volume processing of Doppler ultrasound signals, Ultrasound Med. Biol. 20, 953-965.

Hoeks A.P.G., Hennerici M. and Reneman R.S. (1991): Spectral composition of Doppler signals, Ultrasound Med. Biol. 17, 751-760.

Hoeks A.P.G., Van de Vorst J.J.W., Dabekaussen A., Brands P.J. and Reneman R.S. (1991): An 
efficient algorithm to remove low frequency Doppler signals in digital Doppler systems, Ultrasonic Imaging 13, 135-144.

Hottier F. and Bernatets J.-L. (1984): Estimation of ultrasonic attenuation in biological tissues, Acta Electronica 26, 33-58.

Hunt J.W., Arditi M. and Foster F.S. (1983): Ultrasound transducers for pulse-echo medical imaging, IEEE Trans. Biomed. Eng. 30, 453-481.

Jones J.P. and Leeman S. (1984): Ultrasonic tissue characterization: a review, Acta Electronica 26, 3-31.

Kasai C., Namekawa K., Koyano A. and Omoto R. (1985): Real-time two-dimensional blood flow imaging using an autocorrelation technique, IEEE Trans. Sonics Ultrasonics 32, 458-464.

Kristoffersen K. (1986): Optimal receiver filtering in pulsed Doppler ultrasound blood velocity measurement, IEEE Trans. Ultrason. Ferro. Freq. Control 33, 51-58.

Leavitt S.C., Hunt B.F. and Larsen H.G. (1983): A scan conversion algorithm for displaying ultrasound images, Hewlett-Packard Journal 10, 30-34.

Lee M.H., Kim J.H. and Park S.B. (1986): Analysis of a scan conversion algorithm for a real-time sector scanner, IEEE Trans. Med. Imaging 5, 96-105.

Lee S.W. and Park S.B. (1985): A new scan conversion algorithm for ultrasound compound scanning, Ultrasonic Imaging 7, 215-224.

Levkowitz H. and Herman G.T. (1993): GLHS: a generalized lightness, hue and saturation color model, CVGIP-Graph. Mod. Im. 55, 271-285.

Loupas T. and McDicken W.N. (1990): Low-order complex AR models for mean and maximum frequency estimation in the context of Doppler color flow mapping, IEEE Trans. Ultrason. Ferro. Freq. Control 37, 590-601.

Lu J.Y., Zou H. and Greenleaf J.F. (1994): Biomedical ultrasound beam forming, Ultrasound Med. Biol. 20, 403-428.

Neppiras E.A. (1984): Acoustic cavitation, an introduction, Ultrasonics 22, 25-28.

Newhouse V.L., Bendick P.J. and Varner L.W. (1976): Analysis of transit time effects on Doppler flow measurement, IEEE Trans. Biomed. Eng. 23, 381-387.

Oosterveld B.J., Thijssen J.M., Hartman P.C., Romijn R.L. and Rosenbusch G.J.E. (1991): Ultrasound attenuation and texture analysis of diffuse liver disease: methods and preliminary results, Phys. Med. Biol. 36, 1039-1064.

Ophir J. and Maklad N.F. (1979): Digital scan converters in diagnostic ultrasound imaging, Proc. IEEE 67, 654-664. 
Parker J.A., Kenyon R.V. and Troxel D.E. (1983): Comparison of interpolating methods for image resampling, IEEE Trans. Med. Imaging 2, 31-39.

Peronneau P.A., Bournat J.-P., Bugnon A., Barbet A. and Xhaard M. (1974): Theoretical and practical aspects of pulsed Doppler flowmetry: real-time application to the measure of instantaneous velocity profiles in vitro and in vivo, in Cardiovascular applications of ultrasound, Reneman R.S., Ed., North-Holland Publishing Company, Amsterdam, pp. 66-84.

Powis R.L. and Schwartz R.A. (1991): Practical Doppler Ultrasound for the Clinician (Williams \& Wilkins, Baltimore).

Rabiner L.R. and Gold B. (1975): Theory and Application of Digital Signal Processing (PrenticeHall, New Jersey).

Richard W.D. and Arthur R.M. (1994): Real-time ultrasonic scan conversion via linear interpolation of oversampled vectors, Ultrasonic Imaging 16, 109-123.

Robinson D.E. and Knight P.C. (1982): Interpolation scan conversion in pulse-echo ultrasound, Ultrasonic Imaging 4, 297-310.

Steward E.G. (1983): Fourier Optics: an Introduction (Ellis Horwood, Chichester).

Sung S.H. (1989): Acoustic wave-field analysis of ultrasonic transducers, J. Acoust. Soc. Am. 86, 1595-1601.

Takeuchi Y. (1987): Color coded Doppler scans, Ultrasound Med. Biol. 13, 151-152.

Ter Haar G.R. (1986): Ultrasonic biophysics, in Physical principles of medical ultrasonics, Hill C.R., Ed., Ellis Horwood, Chichester, pp. 378-435.

Thijssen J.M., Oosterveld B.J. and Wagner R.F. (1988): Gray level transforms and lesion detectability in echographic images, Ultrasonic Imaging 10, 171-195.

Van Leeuwen G.H., Hoeks A.P.G. and Reneman R.S. (1986): Simulation of real-time frequency estimators for pulsed Doppler systems, Ultrasonic Imaging 8, 252-271.

Wells P.N.T. (1969): Physical Principles of Ultrasonic Diagnosis (Academic Press, London).

Wells P.N.T. (1977): Biomedical Ultrasonics (Academic Press, London).

Wells P.N.T. (1986): The prudent use of diagnostic ultrasound, Br. J. Radiol. 59, 1143-1151.

Wells P.N.T., De Jong N., Bom N. and Somer J. (1986): Transducers in medical ultrasound. Part 4: transducer safety, Ultrasonics 24, 230-232.

Wilson L.S. (1991): Description of broad-band pulsed Doppler ultrasound processing using the twodimensional Fourier transform, Ultrasonic Imaging 13, 301-315. 
Zemanek J. (1971): Beam behavior within the nearfield of a vibrating piston, J. Acoustical Soc. Am. 49, 181-191. 



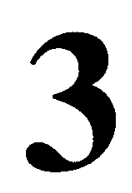

\section{Information Limitations and Human Vision}


Chapter 3 
The amount of information on organ anatomy and blood flow behavior the human observer gains by exploring real-time ultrasound images, is restricted. On the one hand, the physical characteristics of ultrasound pose a limit to the detail to which targets can be interrogated. On the other hand, the properties of the human visual system limit the extent to which the information displayed can be absorbed. For an optimum performance of the combination imaging device - observer, the data display format should match (as far as possible) the characteristics of human visual perception (Sharp, 1993). That is the point where signal postprocessing may play a role. As an introduction to Chapters 4 to 6 , the limitations of B-mode echo imaging and the real-time display of color velocity profiles are investigated.

\subsection{B-mode echo imaging}

According to Hill et al. (1991), the qualification of a B-mode imaging system is comprised by three quantities: spatial resolution, temporal resolution and contrast discrimination. Spatial resolution, as defined in section 2.3 , is determined by the size of the echo sample volume, which in its turn is determined by the local volume of tissue occupied by the ultrasound pulse emitted and the sensitivity pattern of the transducer in reception mode. The shape of the sample volume gradually modifies with distance due to the various mechanisms acting on the sound wave propagation in tissue. As a consequence, spatial resolution too varies over the image. Wellknown techniques aimed at the improvement of the spatial resolution throughout the plane of observation are dynamic focusing, electronically forcing the focus of a multiple element transducer to move in reception mode along with the echo sample volume into depth, phase aberration correction (Nock et al., 1989), adaptively correcting the delays of a multiple element transducer to compensate for the differences in propagation speed encountered when different parts of the ultrasound pulse travel through different types of tissue, and deconvolution (Jeurens et al., 1987), attempting to recover the spatial distribution of microscopic acoustic inhomogeneities by deconvolving the rf signals (Eq. (2.16)) with an estimate of the spatial rf point spread function. Whereas dynamic focusing is commonly applied in modern commercial equipment, phase aberration correction is still in development. Deconvolution has only been of limited importance in practice, mainly due to the complex, space varying shape of the ultrasound pulse. Some auxiliary approaches to improve spatial resolution were summarized by Harris et al. (1991).

Temporal resolution may be defined as the number of separate $r f$ frames recorded per time interval, which corresponds to the frame rate FR. According to Eqs. (2.17) and (2.18), the frame rate is ultimately limited by the speed of ultrasound 
and the depth of penetration. Typical frame rates range between 10 and 30 per second. Experimental techniques have been described (Shattuck et al., 1984) that substantially increase frame rate, at some expense of spatial resolution, by simultaneously recording rf signals along a number of separate scan lines. In these methods, a relatively wide ultrasound beam is used in emission mode to illuminate at once a large area in the plane of observation. In reception mode, echoes are simultaneously caught along a number of narrow, independent sensitivity regions.

Contrast discrimination refers to the capacity of the ultrasound system to generate distinct images (in mean brightness) of macroscopic homogeneous targets differing exclusively in the mean echo amplitude level they produce. Ultimately, contrast discrimination is limited by the size of the bins into which the gray scale is divided, bin size being expressed as the range of input echo amplitude levels eventually mapped onto a single gray value. Appropriate assignment of gray levels by means of a carefully chosen gray level transform (section 2.3.3), such that the gray level bins are small within the amplitude range of interest and larger outside, can therefore optimize contrast discrimination. In that case, contrast resolution, i.e., the minimum difference in mean echo amplitude level between two homogeneous image regions that can be detected, is predominantly determined by the spatial resolution of the system and the image noise present. Whereas electronic and quantization noise restrict the depth of investigation, acoustic noise or speckle is the principal limiting factor of contrast resolution. Before further expanding on contrast resolution, a brief excursion is made into the nature of speckle.

Speckle results from the coherent summation at the transducer surface of ultrasound echoes coming from tiny acoustic inhomogeneities (scatterers) too close to be separately resolved, and gives the B-mode image a typical granular, mottled appearance. Speckle is, strictly spoken, not true random noise, as it is the result of a deterministic process in which ultrasound echoes interfere with each other. However, if scatter density is sufficiently high (of the order of ten scatterers per echo sample volume or more (Oosterveld et al., 1985; Tuthill et al., 1988; Wilhelmij and Denbigh, 1984)), speckle texture is no longer related to the spatial distribution of scatterers but solely depends on transducer geometry and the characteristics of the transient ultrasound field established. Theoretical analysis of the spatial amplitude pattern associated with this situation of fully developed speckle shows, that speckle behaves like multiplicative noise, whereby the first-order statistical properties (at a single point in space) of the amplitude pattern in response to a macroscopic homogeneous target, are described by a Rayleigh probability density function (Burckhardt, 1978; Goodman, 1975; Wagner et al., 1983): 


$$
\mathrm{p}(\mathrm{A})=\frac{\mathrm{A}}{\psi} \cdot e^{-\frac{\mathrm{A}^{2}}{2 \psi}} \quad(\mathrm{A} \geq 0)
$$

where $\psi=\overline{A^{2}} / 2$ depends on the mean square scattering amplitude of the particles. The Rayleigh distribution of Eq. (3.1) is characterized by the ratio of the mean amplitude level (the signal) and the standard deviation (the noise), also referred to as the point signal-to-noise ratio:

$$
\operatorname{SNR}_{\mathrm{A}} \stackrel{\operatorname{def}}{=} \frac{\bar{A}}{\sigma_{\mathrm{A}}} \approx 1.91
$$

The reciproke point signal-to-noise ratio $\sigma_{A} / \bar{A}$ is referred to as the speckle contrast.

The second-order statistics of the amplitude pattern (reflecting the noise correlations between different points in space) are summarized by the mean axial and lateral speckle cell sizes, defined as the areas under the normalized one-dimensional autocovariance functions in the respective two directions (or, alternatively, as the respective FWHMs). Assuming the axial pulse envelope to be Gaussian with a standard deviation of $\sigma_{y}[\mathrm{~mm}]$, the average speckle size in the focal zone of a piston transducer is approximated by (Smith and Wagner, 1984; Wagner et al., 1988; Wagner et al., 1983):

$$
\begin{array}{ll}
\mathrm{S}_{\mathrm{y}} \approx 2.43 \sigma_{\mathrm{y}} \approx 1.02 \mathrm{FWHM}_{\text {pulse envelope }} & {[\mathrm{mm}]} \\
\mathrm{S}_{\mathrm{x}} \approx 0.87 \frac{\lambda \cdot \mathrm{F}}{\mathrm{D}^{\prime}} & {[\mathrm{mm}]}
\end{array}
$$

where $D^{\prime}$ equals the circular transducer aperture diameter $2 a$ divided by 1.08 . It follows that the average speckle cell size is comparable to the local resolution cell size of the ultrasound system.

The definition of contrast resolution involves the specification of an observer and a suitable signal detection task. A standard problem is to decide whether or not a contrasting disk (a circular lesion) of known size is present at a specified location in an otherwise homogeneous B-mode image (a so-called signal specified exactly problem). Signal detection theory predicts that the best possible performance in terms of a percentage correct decisions is exhibited by that observer that employs all information at his disposal and that uses a (monotonic function of) likelihood ratio as a decision criterion (Green and Swets, 1988). In this context lesion information is 
assumed to be available in the form of a number of independent, peak detected amplitude points equal to the number of independent speckle cells in the area suspected, or, different, in the form of a signal level as averaged over the region of interest. A positive relationship was indicated to exist between the performance of the ideal observer and the contrast of the lesion (i.e., the absolute difference between the average lesion and background amplitude levels relative to the sum) as well as the square root of the number of speckle cells in the lesion (Smith et al., 1983; Thijssen et al., 1988). The latter dependency on speckle density implies that contrast resolution and spatial resolution are inextricably linked. Therefore, lesion detectability can be improved by appropriate beam shaping, locally increasing spatial resolution by minimizing the echo sample volume and enhancing lesion contrast by suppressing side lobes (Moshfeghi, 1987). In addition, spatial compounding, superimposing a number of uncorrelated ultrasound images of the same object, has been suggested as a way to improve the detectability of contrasting structures (Smith et al., 1983). Further, gray level transformation (Thijssen et al., 1988) and various signal estimation techniques (Kotropoulos and Pitas, 1992; Verhoeven and Thijssen, 1993) have been studied in the context of lesion detection.

Concerning the human observer, results indicate that his performance in lesion detection is less than but still related to the behavior of the ideal observer (Smith et al., 1983), although reproducibility was found to be a problem in these experiments (Lopez et al., 1990). An obvious limitation of the concept of the ideal observer in this respect is, that the size and shape of the lesion as well as the exact location in the image where it could show up, are assumed to be known on forehand. In medical practice, of course, these data are not available. Moreover, the influence of anatomical information in the image remains unclear. Therefore, in searching for the optimum way to communicate the B-mode image, it is necessary to account for the characteristics of human vision.

The human visual system, consisting of the eyes, part of the brain (visual cortex) and pathways connecting the eyes to the visual cortex, faces the awkward task to recognize and interpret subtle gray level contrast differences in the presence of speckle noise. Some evidence exists that (part of) human vision acts, to a certain extent, as a spatial frequency filter with a maximum sensitivity to frequencies of the order of a few cycles per degree and with a fall-off at both higher and lower frequencies (Cornsweet, 1970). This implies a maximum sensitivity of human vision to typical speckle noise patterns at normal viewing distance (Bamber, 1993). Consequently, speckle is likely to distract the viewer, this way impeding the transfer of relevant diagnostic information. Replacement of the gray level look-up table by a pseudo color bar might make this effect even stronger and seems 
therefore in general not most suitable to improve the visibility of small contrast differences. An alternative is signal averaging, reducing speckle contrast while sacrificing some resolution in space or time. Concrete methods are frequency compounding (Magnin et al., 1982; Melton Jr. and Magnin, 1984; Trahey et al., 1986), frame averaging (Bartrum Jr. and Crow, 1980) and (adaptively) low pass filtering of the image (Bamber and Daft, 1986; Bleck et al., 1994; Dickinson, 1982; Koo and Park, 1991; Lopes et al., 1990; Loupas et al., 1989; Loupas et al., 1987) The method to be described in Chapter 5 reduces speckle contrast too, but is unconventional in the way spatial amplitude information is averaged. The experimental results to be presented reveal that the novel method is superior to standard low pass filtering in lesion detection.

An alternative approach to speckle reduction is proposed in Chapter 4. In this method, $S N R_{A}$ is hardly influenced, i.e., speckle noise power is preserved with respect to signal power. Instead, noise bandwidth is increased by introducing a noiserelated distortion, causing part of the noise power to be shifted away from the frequencies of visual peak sensitivity. The result is a crisp image that may be perceived as exhibiting reduced speckle contrast. An objective reduction of speckle contrast can be achieved by applying a spatial low pass filter afterwards: the enlarged noise bandwidth results in an increased noise reduction as compared with filtering of the original image.

An essential step in the process of image interpretation by man seems the detection of edges. Even on the lowest level of human vision a mechanism is likely to exist in which retina cell interaction leads to the enhancement of object boundaries in the retinal image, facilitating edge localization and explaining the perception of Mach bands (Cornsweet and Yellot Jr., 1985; Levine, 1985). The obvious importance of edges has led to the development of various image postprocessing algorithms to emphasize structure boundaries. Regarding B-mode ultrasound images, edge enhancement is hampered by speckle noise, which gives easily rise to the detection of false edges. A way to cope with this problem is proposed in Chapter 4 . The above introduced speckle reduction algorithm of this chapter will be shown to be extendable to enhance edges in all directions. The extended algorithm could lead to an improved visibility of small vessels that lack a clear definition in the original image, such as liver veins. In addition, the algorithm might be of value in vessel wall tracking applications, to determine automatically the initial positions of the anterior and posterior artery wall - lumen interfaces in the presence of reverberations (Hoeks et al., 1995). 


\subsection{Real-time display of color velocity images}

Practice has learned that the temporal resolution of B-mode ultrasound systems is generally sufficient to give the human observer the illusion of a smooth and continuous flow of echographic information in time. The cinematographic effect allows the viewer to employ coherent object motion as an aid in the detection of structures corrupted by noise. In color velocity imaging, however, low frame rates (of the order of 10 [s $\left.\mathrm{s}^{-1}\right]$ or less) can be problematic, especially under dynamic blood flow conditions. Under these circumstances, the generation of inter frames by means of interpolation may be helpful to keep image display rate at an acceptable level. Still, the rather frayed appearance of color velocity profiles (section 2.5.1) remains a problem in real-time display. Taking into account that human vision has been found to be maximum sensitive to temporal frequencies between about 10 and 30 cycles per second (Kelly, 1961), the irregular velocity profile boundaries, rapidly altering from frame to frame in an unpredictable fashion, are likely to distract the observer. This effect can be reduced by employing a low pass filter (generally known as persistence filter) to smooth the temporal velocity signal of each velocity pixel while it is progressing over consecutive frames. A side effect is, that all true velocity information measured is also influenced, and especially instantaneous peak velocities become masked. In Chapter 6 an alternative algorithm is proposed, which has equal capacity as a standard persistence filter to improve the consistency in a sequence of color velocity profiles, but which lacks the side effects of velocity smoothing.

\section{REFERENCES}

Bamber J.C. (1993): Speckle reduction, in Advances in ultrasound techniques and instrumentation, Wells P.N.T., Ed., Churchill Livingstone, New York, pp. 55-67.

Bamber J.C. and Daft C. (1986): Adaptive filtering for reduction of speckle in ultrasonic pulse-echo images, Ultrasonics $24,41-44$.

Bartrum Jr. R.J. and Crow H.C. (1980): Ultrasound echo averaging: A simple method for improving image perception, J. Clin. Ultrasound 8, 63-64.

Bleck J.S., Gebel M., Hebel R., Wagner S., Schmidt K., Kruip S., Westhoff-Bleck M., Wolf M., Thiesemann C. and Manns M. (1994): Tissue characterization using intelligent adaptive filter in the diagnosis of diffuse and focal liver disease, Ultrasound Med. Biol. 20, 521-528.

Burckhardt C.B. (1978): Speckle in ultrasound B-mode scans, IEEE Trans. Sonics Ultrasonics 25, 16. 
Cornsweet T.N. (1970): Visual Perception (Academic Press, New York).

Cornsweet T.N. and Yellot Jr. J.I. (1985): Intensity-dependent spatial summation, J. Opt. Soc. Am. A 2, 1769-1786.

Dickinson R.J. (1982): Reduction of speckle in ultrasound B-scans by digital processing, in Acoustical Imaging 12, Ash E.A. and Hill C.R., Eds,, Plenum Press, New York, pp. 213-224.

Goodman J.W. (1975): Statistical properties of laser speckle patterns, in Laser speckle and related phenomena, Dainty J.C., Ed., Springer-Verlag, Berlin, pp. 9-75.

Green D.M. and Swets J.A. (1988): Signal Detection Theory and Psychophysics (Peninsula Publishing, Los Altos).

Harris R.A., Follett D.H., Halliwell M. and Wells P.N.T. (1991): Ultimate limits in ultrasonic imaging resolution, Ultrasound Med. Biol. 17, 547-558.

Hill C.R., Bamber J.C., Crawford D.C., Lowe H.J. and Webb S. (1991): What might image echography learn from image science?, Ultrasound Med. Biol. 17, 559-575.

Hoeks A.P.G., Di X., Brands P.J. and Reneman R.S. (1995): An effective algorithm for measuring diastolic artery diameters, Archives of Acoustics 20, 65-76.

Jeurens T.J.M., Somer J.C., Smeets F.A.M. and Hoeks A.P.G. (1987): The practical significance of two-dimensional deconvolution in echography, Ultrasonic Imaging 9, 106-116.

Kelly D.H. (1961): Visual responses to time-dependent stimuli. I. Amplitude sensitivity measurements, J. Opt. Soc. Am. 51, 422-429.

Koo J.I. and Park S.B. (1991): Speckle reduction with edge preservation in medical ultrasonic images using a homogeneous region growing mean filter (HRGMF), Ultrasonic Imaging 13, 211-237.

Kotropoulos C. and Pitas I. (1992): Optimum nonlinear signal detection and estimation in the presence of ultrasonic speckle, Ultrasonic Imaging 14, 249-275.

Levine M.D. (1985): Vision in Man and Machine (McGraw-Hill, New York).

Lopes A., Touzi R. and Nezry E. (1990): Adaptive speckle filters and scene heterogeneity, $I E E E$ Trans. Geoscience Remote Sensing 28, 992-1000.

Lopez H., Loew M.H,, Butler P.F., Hill M.C. and Allman R.M. (1990): A clinical evaluation of contrast-detail analysis for ultrasound images, Med. Phys. 17, 48-57.

Loupas T., McDicken W.N. and Allan P.L. (1989): An adaptive weighted median filter for speckle suppression in medical ultrasonic images, IEEE Trans. Circuits Systems 36, 129-135.

Loupas T., McDicken W.N. and Allen P.L. (1987): Noise reduction in ultrasonic images by digital filtering, Br. J. Radiol. 60, 389-392. 
Magnin P.A., Von Ramm O.T. and Thurstone F.L. (1982): Frequency compounding for speckle contrast reduction in phased array images, Ultrasonic Imaging 4, 267-281.

Melton Jr. H.E. and Magnin P.A. (1984): A-mode speckle reduction with compound frequencies and compound bandwidths, Ultrasonic Imaging 6, 159-173.

Moshfeghi M. (1987): Side-lobe suppression for ultrasonic imaging arrays, Ultrasonics 25, 322-327.

Nock L., Trahey G.E. and Smith S.W. (1989): Phase aberration correction in medical ultrasound using speckle brightness as a quality factor, J. Acoust. Soc. Am. 85, 1819-1833.

Oosterveld B.J., Thijssen J.M. and Verhoef W.A. (1985): Texture of B-mode echograms: 3-D simulations and experiments of the effects of diffraction and scatterer density, Ultrasonic Imaging 7, 142160.

Sharp P.F. (1993): Display and perception of ultrasound images, in Advances in ultrasound techniques and instrumentation, Wells P.N.T., Ed., Churchill Livingstone, New York, pp. 1-18.

Shattuck D.P., Weinshenker M.D., Smith S.W. and Von Ramm O.T. (1984): Explososcan: A parallel processing technique for high speed ultrasound imaging with linear phased arrays, J. Acoust. Soc. Am. 75, 1273-1282.

Smith S.W. and Wagner R.F. (1984): Ultrasound speckle size and lesion signal to noise ratio: verification of theory, Ultrasonic Imaging 6, 174-180.

Smith S.W., Wagner R.F., Sandrik J.M. and Lopez H. (1983): Low contrast detectability and contrast/detail analysis in medical ultrasound, IEEE Trans. Sonics Ultrasonics 30, 164-173.

Thijssen J.M., Oosterveld B.J. and Wagner R.F. (1988): Gray level transforms and lesion detectability in echographic images, Ultrasonic Imaging 10, 171-195.

Trahey G.E., Allison J.W., Smith S.W. and Von Ramm O.T. (1986): A quantitative approach to speckle reduction via frequency compounding, Ultrasonic Imaging 8, 151-164.

Tuthill T.A., Sperry R.H. and Parker K.J. (1988): Deviations from Rayleigh statistics in ultrasonic speckle, Ultrasonic Imaging 10, 81-89.

Verhoeven J.T.M. and Thijssen J.M. (1993): Improvement of lesion detectability by speckle reduction filtering: a quantitative study, Ultrasonic Imaging 15, 181-204.

Wagner R.F., Insana M.F. and Smith S.W. (1988): Fundamental correlation lengths of coherent speckle in medical ultrasonic images, IEEE Trans. Ultrason. Ferro. Freq. Control 35, 34-44.

Wagner R.F., Smith S.W., Sandrik J.M. and Lopez H. (1983): Statistics of speckle in ultrasound Bscans, IEEE Trans. Sonics Ultrasonics 30, 156-163.

Wilhelmij P. and Denbigh P. (1984): A statistical approach to determining the number density of random scatterers from backscattered pulses, J. Acoust. Soc. Am. 76, 1810-1818. 


\section{4}

\section{Ultrasonic B-mode Image Enhancement}

based on

\section{Level-Dependent Spread Functions}

Chapter 4 is based on the publication:

R.J. Collaris and A.P.G. Hoeks (1994): Ultrasonic B-mode image enhancement based on level-dependent spread functions, Ultrasonic Imaging 16, 205-230. 
Chapter 4 


\section{List of symbols}

Symbols associated with signal distributions:

$x, y$

space coordinates. In the context of ultrasonic imaging, $x$ denotes lateral position and $y$ denotes axial position, both expressed in sample points.

$A(x, y) \quad$ nonnegative signal distribution.

$M(x, y)$

output of the LDS algorithm (distribution of Mach bands).

$N(x, y)$ additional output of the extended LDS algorithm, which includes for every point $(x, y)$ the number of times the location was covered by a spread function.

$\operatorname{Out}(x, y)$ overall output of the algorithm for image enhancement, including the distribution of processed amplitudes.

Symbols associated with level-dependent spatial summation:

C

q

$S_{L D, 0}\left((x, y) ; C A^{q}\right)$

$S_{L D}\left(\left(x-x^{\prime}, y-y^{\prime}\right) ; c A^{q}\right)$

$d_{x}, d_{y}$

o

$x_{\mathrm{S}}$

\section{LDS scaling constant.}

power to which a signal level is raised for modulation of a spread function.

level-dependent convolution kernel with height 1 , enclosing a volume equal to $1 / c A^{q}$.

level-dependent spread function centered around point $\left(x^{\prime}, y^{\prime}\right)$, with height $c A^{q}$, enclosing a volume equal to 1 .

radius of support in $x$ and $y$ direction, respectively.

base area of a spread function with a finite radius of support in all directions.

the location farthest from the foot of an edge where the LDS output still exceeds 1 .

Symbols associated with the echo system:

$f_{S, A}$

BW

$S$

$S_{\mathrm{ff}}$ envelope sampling frequency.

rf signal bandwidth.

average axial or lateral speckle cell size, measured as the area below the normalized 1D autocovariance function of the spatial amplitude distribution in the corresponding direction (down to 10 percent of its maximum).

speckle cell size as measured from foot to foot, which equals approximately two times the average cell size $S$. 
Symbols associated with the algorithm for ultrasonic image enhancement:

$N_{\max }$
$\bar{N}_{\text {speckle }}$
$T_{N}$
$A_{\text {eff }}$
$C_{1-2}, C_{L}$
$p_{s}$
$p_{e}$
$p_{c}$

\subsection{Abstract}

The concept of level-dependent spatial summation is applied to the two-dimensional amplitude distribution comprising an ultrasonic brightness image. The distribution is convolved with a level-dependent kernel. The size (i.e. extent) of the kernel is inversely proportional to the local signal level. The result is a two-dimensional distribution of Mach bands, accentuating edges and providing useful information for the reduction of speckle. In addition, a small but powerful extension of the processing scheme is presented that supports global edge enhancement and contrast enhancement. The level of improvement has been quantified in terms of an increase in the lesion signal-to-noise ratio of the order of 60 percent. Two system-dependent parameters have to be supplied for adjustment. The algorithm is rather insensitive to the exact choice of the parameters, making it quite robust. Since only a limited number of simple operations is required for the processing of a frame, the algorithm is expected to be suitable for real-time application.

Key words: contrast enhancement; edge enhancement; image enhancement; leveldependent spatial summation; speckle reduction; spread function.

\subsection{Introduction}

Over the last decade, a great deal of research has been devoted to signal postprocessing techniques that enhance ultrasonic B-mode images. In this context, image enhancement is normally interpreted as speckle reduction with preservation of relevant image features. Various filtering techniques have been suggested to cope with 
this difficult task (Bamber and Daft, 1986; Dickinson, 1982; Koo and Park, 1991; Kotropoulos and Pitas, 1992; Loupas et al., 1989; Verhoeven and Thijssen, 1993). The adaptive algorithms proposed all are variations on the same basic idea: local firstorder statistics can be used to adjust the filter's characteristics such that areas containing speckle are smoothed and areas containing structures are preserved. As a result, speckle contrast $\left(=1 / S N R_{A}=\sigma_{A} / \bar{A}\right)$, conform Eq. (3.2)) is reduced, facilitating the perception of local mean brightness and improving image quality objectively (Verhoeven and Thijssen, 1993) and, after a learning process, also subjectively (Crawford et al., 1992). That the smooth appearance of processed images is not always comfortable to the unfamiliarized observer, may be explained by the fact that smoothed speckle tends to give the viewer a false idea of blurredness, of a lack of focus. Consequently, he may experience the processed images as if some information loss had occurred, although ideally, this is not the case.

In the present study, a different approach to ultrasonic image enhancement is proposed. In this method, speckle is not smoothed (the image is not blurred) and the first-order statistical parameter speckle contrast is slightly affected, which indicates that speckle noise power is preserved with respect to mean signal power. Essentially, the present processing scheme redistributes speckle noise power over an increased bandwidth. This way, the average speckle size is reduced and the density of linearly independent points is increased. As argued in Chapter 3 , the resulting crisp image may be perceived as exhibiting reduced speckle contrast, depending on image magnification and viewing distance, which may facilitate the recognition of slight differences in local mean brightness in the image. Moreover, application of speckle reduction prior to low pass filtering makes the latter operation more effective (i.e., reduction of speckle contrast is increased in accordance with the increased number of independent points over which is averaged). A powerful extension of the algorithm supports the enhancement of global edges (especially, the transition from vessel wall to lumen) and the enhancement of contrast between tissues having different mean reflectivities, such as lesions against a background. Because of its intrinsic simplicity, the algorithm is expected to be suitable for real-time application.

The basic idea in the method is the application of level-dependent spatial summation (LDS) to the two-dimensional distribution of envelope signals comprising a B-mode image. LDS is a generalization of intensity-dependent spatial summation (IDS), which was introduced by Cornsweet and Yellot Jr. in 1985. They presented IDS as a new model for the interaction of cells in the retina. IDS appears to be a very attractive model since, though very simple, it can not only predict the increase in visual acuity with increasing mean luminance (as expected), but it also explains the 
perception of the so-called Mach bands (first described by Ernst Mach in 1865) at the edges of objects having different brightness levels. It will be demonstrated that the concept of LDS can be applied successfully to the two-dimensional ultrasound envelope distribution. Moreover, the algorithm is extended to enhance region contrast and global edges in the image.

\subsection{Level-dependent spatial summation}

\subsubsection{Method}

A brief description of the LDS algorithm will be presented and some of its basic properties will be pointed out. A more exhaustive treatment of the theory can be found in Cornsweet and Yellot Jr. (1985) and Reese (1992). The LDS algorithm operates on a nonnegative signal distribution $A$, which can be one-dimensional or twodimensional. It basically performs a convolution of $A$ with a level-dependent kernel $S_{L D, O}$. The output $M(x, y)$ is determined by:

$$
M(x, y)=\sum_{x^{\prime}} \sum_{y^{\prime}} c A^{q}\left(x^{\prime}, y^{\prime}\right) \cdot s_{L 0,0}\left(\left(x-x^{\prime}, y-y^{\prime}\right) ; c A^{q}\left(x^{\prime}, y^{\prime}\right)\right)
$$

where $c$ is the LDS scaling constant and $q$ is a positive exponent. Kernel $s_{L D, O}\left((x, y) ; c A^{q}\right)$ has the following fundamental properties:

1. It is nonnegative.

2. It is centered around the origin.

3. It takes its largest value at the origin, where it equals 1.

4. It encloses a volume equal to $1 / C A^{q}$.

The product $c A^{q}\left(x^{\prime}, y^{\prime}\right) \cdot s_{L D, O}\left(\left(x-x^{\prime}, y-y^{\prime}\right) ; c A^{q}\left(x^{\prime}, y^{\prime}\right)\right)$ defines a level-dependent spread function $s_{L D}\left(\left(x-x^{\prime}, y-y^{\prime}\right) ; c A^{q}\left(x^{\prime}, y^{\prime}\right)\right)$, determining the contribution of the input $A$ at location $\left(x^{\prime}, y^{\prime}\right)$ to the output $M$ at position $(x, y)$. Essentially, the ratio between height and extent of the spread function is modulated by the local signal level while the volume enclosed by the function remains one. Clearly, high signal levels produce high spread functions with narrow bases and low levels produce low spread functions with wide bases. Normally, spread functions have circular symmetry, but that is not essential for the functioning of the algorithm. In fact, circular symmetry may even be inappropriate, especially in ultrasonic imaging, as will be discussed later. Amplitude distributions, intensity distributions and any other nonnegative distribution can serve as input for the LDS algorithm. 
Examples of spread functions are the Gaussian:

$$
s_{\mathrm{LD}}\left(\left(x-x^{\prime}, y-y^{\prime}\right) ; C A^{q}\left(x^{\prime}, y^{\prime}\right)\right)=\frac{C A^{q}\left(x^{\prime}, y^{\prime}\right)}{2 \pi} e^{-\frac{c A^{q}\left(x^{\prime}, y^{\prime}\right)\left(\left(x-x^{\prime}\right)^{2}+\left(y-y^{\prime}\right)^{2}\right)}{2}}
$$

and the cylindrical:

$$
s_{L D}\left(\left(x-x^{\prime}, y-y^{\prime}\right) ; c A^{q}\left(x^{\prime}, y^{\prime}\right)\right)= \begin{cases}c A^{q}\left(x^{\prime}, y^{\prime}\right) & \text { for } \sqrt{\left(x-x^{\prime}\right)^{2}+\left(y-y^{\prime}\right)^{2}}<d \\ 0 & \text { otherwise }\end{cases}
$$
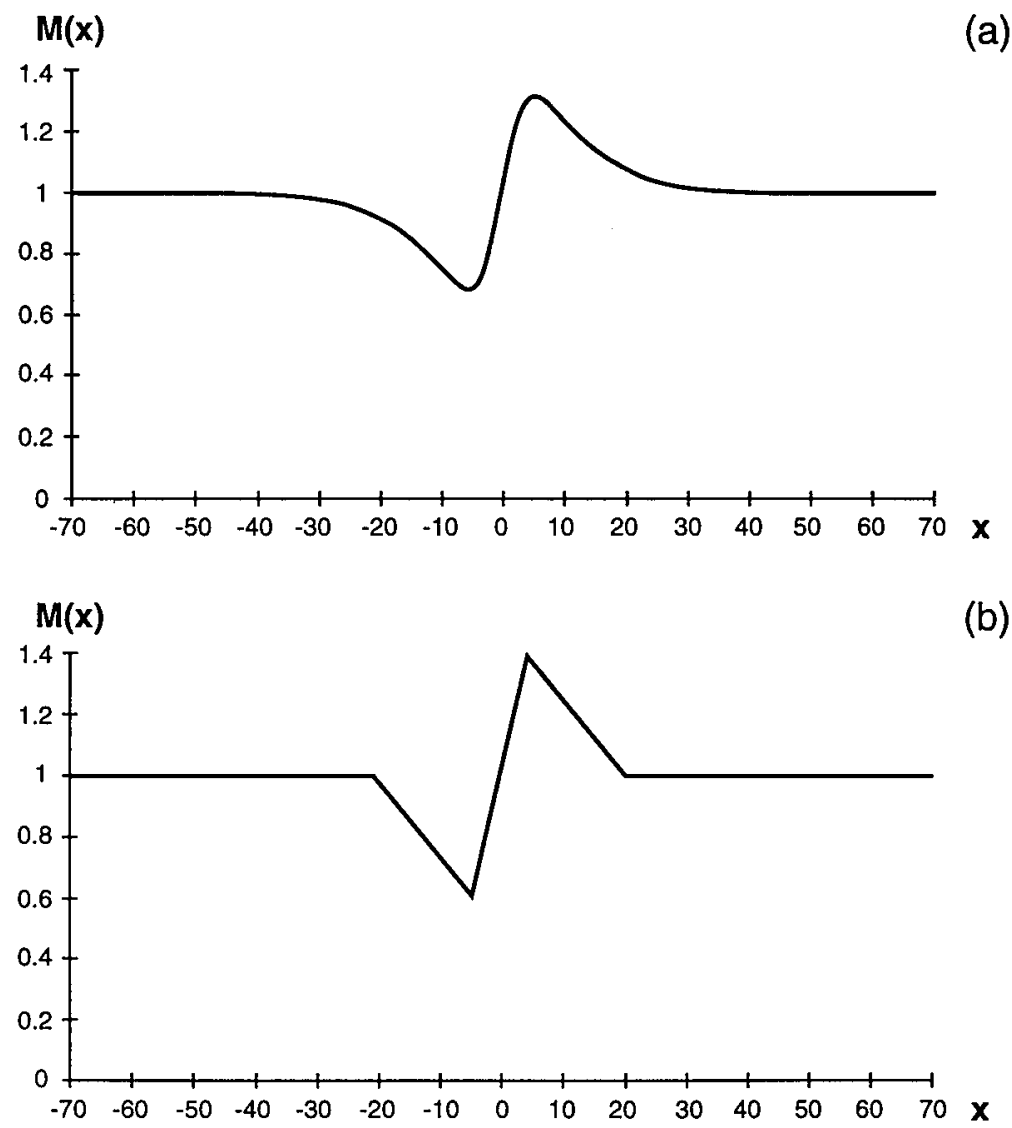

Figure 4.1. Response of the one-dimensional LDS algorithm to a step input for a Gaussian spread function (a) and a rectangular spread function (b). The step at $x=0$ separates a lower signal level at $x<0$ from a higher level at $x>0$. 
where

$$
d=1 / \sqrt{\pi c A^{q}\left(x^{\prime}, y^{\prime}\right)}
$$

is called the "radius of support". The calculation of $M(x, y)$ only requires a limited number of summations, provided that a spread function is applied with a finite radius of support, such as the cylindrical.

Figures 4.1-a and -b show the output of the 1D LDS algorithm to a step input for a Gaussian and a cylindrical spread function, respectively. These figures illustrate the most important properties of the LDS algorithm:

- The response to a constant signal is always 1 , whatever the level.

- Straight edges produce Mach bands: dips in the output at the lower signal level sides and peaks at the higher level sides, accentuating the edges.

The properties shown here are invariant for the type of spread function applied. In the next section, the application to ultrasonic imaging is discussed and the choice of a spread function with a shape according to the system resolution cell is motivated. Thereafter, some of the specific properties of this spread function will be highlighted.

\subsubsection{Application to ultrasonic imaging}

In B-mode echo imaging, pixel brightness is related to echo amplitude. To ensure successful application of the LDS algorithm to spatial amplitude distributions, two conditions should be fulfilled. Firstly, the signals used should closely reflect the actual amplitudes of the corresponding rf signals. This means that simple rectification of the rf signals followed by low pass filtering may not suffice. Instead, it is advised to base amplitude calculations on the Hilbert transform of the rf signals, preferably carried out in real-time applications by a sliding kernel in the time domain (as discussed in section 2.3.1). Secondly, the envelope sampling frequency $f_{s, A}$ should be high enough to ensure a smooth amplitude progression in depth and to allow a precise positioning of the Mach bands. It was found experimentally that about 6 sample points per speckle cell in the axial direction suffice, that is:

$$
\frac{f_{S, A}}{B W} \geq 6
$$

( $B W$ is the bandwidth of the rf signals). Thus, $f_{s, A}$ is a factor of 3 higher than the 
sample frequency according to the Nyquist theorem. If this condition is satisfied, speckle peaks and troughs will not be missed and the transitions are precisely defined. It is noted that although all information is preserved with standard Nyquist sampling, retrieval of this information is time consuming, since complex operations are required, like the convolution of the envelope signals with a sinc function. It is more practical to oversample the signals. Requirements for the lateral sampling frequency are less stringent. A partial overlapping of the regions insonified by the ultrasound beams of neighboring scan positions ( $x$-positions) is sufficient for a smooth amplitude progression in lateral direction.

What remains to be answered is, what kind of spread function to choose? Since the spread functions are to be applied in real-time ultrasound B-mode imaging, and since the basic properties apply to all types of spread functions, computational cost is the most important criterion. In this respect, a cylindrical function seems most suitable. Its height is constant within its radius of support and equals zero outside. Moreover, the radius of support is always finite, unlike those of other functions like the Gaussian and exponential ones. This implies that additional calculations, to determine where to truncate the function, are not needed. Actually, the computational cost of space varying, infinite extent spread functions can be lowered by the use of predefined look-up tables, but these make substantially higher demands on the system hardware.

In ultrasonic imaging, average axial and lateral speckle cell size usually differ (speckle size being measured as the area under the normalized 1D autocovariance function of the amplitude distribution down to 10 percent of its maximum (Wagner et al., 1983)). Hence, the shape of the cylindrical spread function is transformed into an elliptical function according to: $d_{x} / d_{y}=S_{x} / S_{y}$, with $d_{x} / d_{y}$ the ratio of the lateral and axial axis lengths and $S_{x} / S_{y}$ the ratio of the lateral and axial speckle cell sizes, where $d_{x}, d_{y}, S_{x}$ and $S_{y}$ are expressed in units of sample points. Calculations are simplified if the ellipse is approximated by an ordinary rectangle.

These considerations led to the choice of a block-shaped level-dependent spread function, with a height proportional to the local signal amplitude level (making it an amplitude-dependent spread function) and a rectangular base with width-depth proportions that are determined by the ratio of the lateral and axial speckle cell sizes of the echo system (in sample points). 


\subsubsection{Properties of the block-shaped amplitude- dependent spread function}

Although level-dependent spatial summation is conceptually simple, analytical analysis of the response to some arbitrary input signal may be rather troublesome, even if an ordinary block-shaped spread function is applied. However, for the present purpose, only a few features of the LDS output are important. Relevant are the width of the upgoing Mach band and the parameters that determine this width (the reason for this will become clear in the next section). Again, for the sake of simplicity, analysis is confined to the one-dimensional, continuous case in which a straight edge separates two regions with different amplitude levels $A_{1}$ and $A_{2}\left(A_{1}<A_{2}\right)$, as depicted in figure 4.2-a. The result obtained may afterwards be extrapolated to two dimensions. The general expression for the one-dimensional rectangular spread function becomes:

$$
\mathrm{S}_{\mathrm{LD}}\left(x-x^{\prime} ; c A\left(x^{\prime}\right)\right)= \begin{cases}c A\left(x^{\prime}\right) & \text { for }\left|x-x^{\prime}\right|<d=\frac{1}{2 c A\left(x^{\prime}\right)} \\ 0 & \text { otherwise }\end{cases}
$$

and the corresponding convolution kernel $s_{L D, O}$ is defined by:

$$
\mathrm{S}_{\mathrm{LD}, 0}\left(\mathrm{x} ; \mathrm{CA}\left(\mathrm{x}^{\prime}\right)\right)=\varepsilon\left(x+\frac{1}{2 \mathrm{cA}\left(\mathrm{x}^{\prime}\right)}\right)-\varepsilon\left(x-\frac{1}{2 \mathrm{CA}\left(x^{\prime}\right)}\right)
$$

where $\varepsilon(x)$ denotes the unit step function at $x=0$. Convolving $c A$ with $s_{L D, O}$ yields:

$$
M(x)=\int_{-\infty}^{\infty} c A\left(x^{\prime}\right) \cdot s_{L D, 0}\left(x-x^{\prime} ; C A\left(x^{\prime}\right)\right) d x^{\prime}=M_{1}(x)+M_{2}(x)
$$

with

$$
\begin{aligned}
M_{1}(x) & =\int_{-\infty}^{0} c A_{1} \cdot\left(\varepsilon\left(x-x^{\prime}+\frac{1}{2 c A_{1}}\right)-\varepsilon\left(x-x^{\prime}-\frac{1}{2 c A_{1}}\right)\right) d x^{\prime} \\
& = \begin{cases}1 & \text { for } x<-1 /\left(2 c A_{1}\right) \\
0.5-c A_{1} x & \text { for }-1 /\left(2 c A_{1}\right)<x<1 /\left(2 c A_{1}\right) \\
0 & \text { otherwise }\end{cases}
\end{aligned}
$$

and with

$$
M_{2}(x)=\int_{0}^{\infty} c A_{2} \cdot\left(\varepsilon\left(x-x^{\prime}+\frac{1}{2 c A_{2}}\right)-\varepsilon\left(x-x^{\prime}-\frac{1}{2 c A_{2}}\right)\right) d x^{\prime}
$$



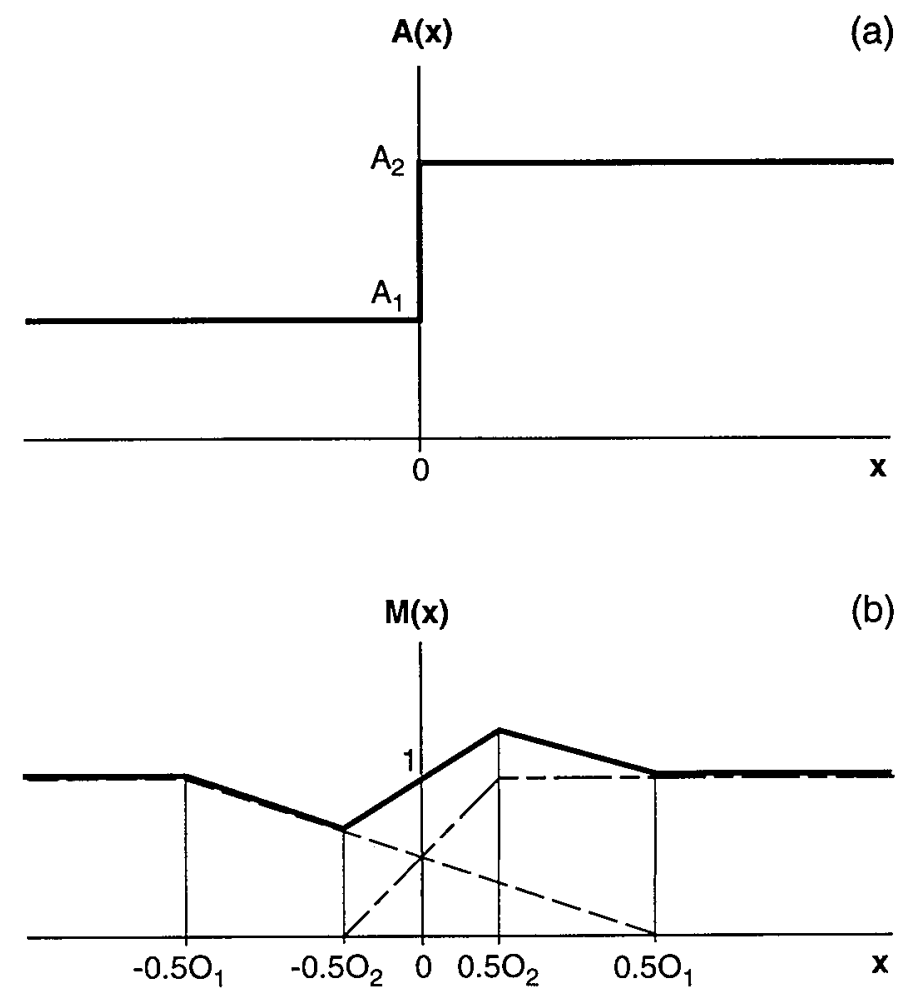

(b)

Figure 4.2. (a) Two signal levels $A_{1}$ and $A_{2}$, separated by a straight edge. (b) The LDS output to the straight edge, with $O_{1}=1 /\left(c A_{1}\right)$ and $O_{2}=1 /\left(c A_{2}\right)$.

$$
= \begin{cases}0 & \text { for } x<-1 /\left(2 c A_{2}\right) \\ c A_{2} x+0.5 & \text { for }-1 /\left(2 c A_{2}\right)<x<1 /\left(2 c A_{2}\right) \\ 1 & \text { otherwise }\end{cases}
$$

Figure 4.2-b shows the output $M(x)$ as the sum of $M_{1}(x)$ and $M_{2}(x)$. It is found that:

- the LDS output is point symmetric around $(0,1)$.

- the peak maximum equals $1.5-0.5 A_{1} / A_{2}$ at $x_{p}=1 /\left(2 c A_{2}\right)$.

- the point $x_{s}$ farthest to the right of the edge where the output still exceeds 1 equals $1 /\left(2 c A_{1}\right)$.

Thus, the LDS scaling constant $c$ determines the exact positions and widths of the Mach bands. 


\subsection{Image enhancement}

\subsubsection{Speckle reduction}

The LDS algorithm can be used to reduce the speckle size and, accordingly, to increase the number of linearly independent points in the image. In sections 4.4.2 and 4.4.3, extensions will be discussed for edge enhancement and contrast enhancement, respectively. The algorithm for speckle reduction operates directly on the raw envelope signals, which are stored in the array $A(x, y)$, where $x$ denotes lateral position (perpendicular to the beam) and $y$ denotes axial position (in depth). The following two processing steps can be distinguished:

1. Calculation of the two-dimensional distribution $M(x, y)$ of Mach bands at edges by means of the LDS algorithm. Edges may be local (at speckle granules) or global (e.g., at the transition between vessel wall and lumen).

2. Making the following decision for each envelope point:

$$
\begin{array}{lll}
\text { if } \quad(M(x, y)>1) & \operatorname{Out}(x, y)=p_{s} \cdot A(x, y) ; \quad\left(0<p_{s}<1\right) \\
\text { else } & \operatorname{Out}(x, y)=A(x, y) ; &
\end{array}
$$

where $\operatorname{Out}(x, y)$ denotes the output array.

In step 2, speckle peaks are lowered while speckle troughs remain unaffected. The principle of the algorithm is illustrated in figure 4.3 for the $1 \mathrm{D}$ case. Input is the harmonic signal: $A(x)=0.5\left(A_{1}+A_{2}\right)+0.5\left(A_{2}-A_{1}\right) \cdot \sin (2 \pi f x)$, where $A_{1}=10$ is the trough value, $A_{2}=50$ is the peak value and $f=0.04$ is the frequency [per sample point]. The foot to foot length of a sine peak equals $1 / f=25$ sample points. Figure 4.3$b$ shows the response of the LDS algorithm for one particular value of the scaling parameter $c(c=0.005)$, which has to be supplied to the LDS algorithm. It is shown that the algorithm produces Mach bands at both sides of every maximum. For the correct functioning of the speckle reduction algorithm, however, the peaks of these Mach bands should more or less coincide, so that the LDS output will continuously exceed 1 , starting from somewhere halfway the leading slope to somewhere halfway the trailing slope of every sine peak. This requirement can be satisfied by lowering $c$ (to 0.002 in figure 4.3-c). But if $c$ becomes too small, the spread functions become so wide for small input values that a peak in the Mach bands distribution produced by one edge may interfere with a dip produced by a different edge, located in the neighborhood. In that case, the LDS output no longer correctly indicates the position of the slopes (see figure 4.3-d, where $c=0.0008$ ). 


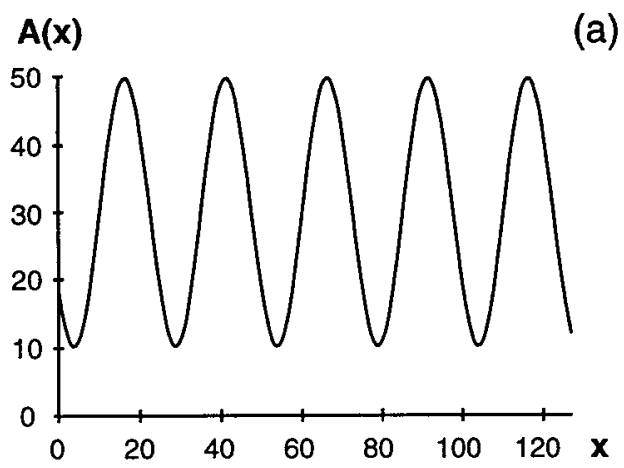

a) $M(x)$

(b)
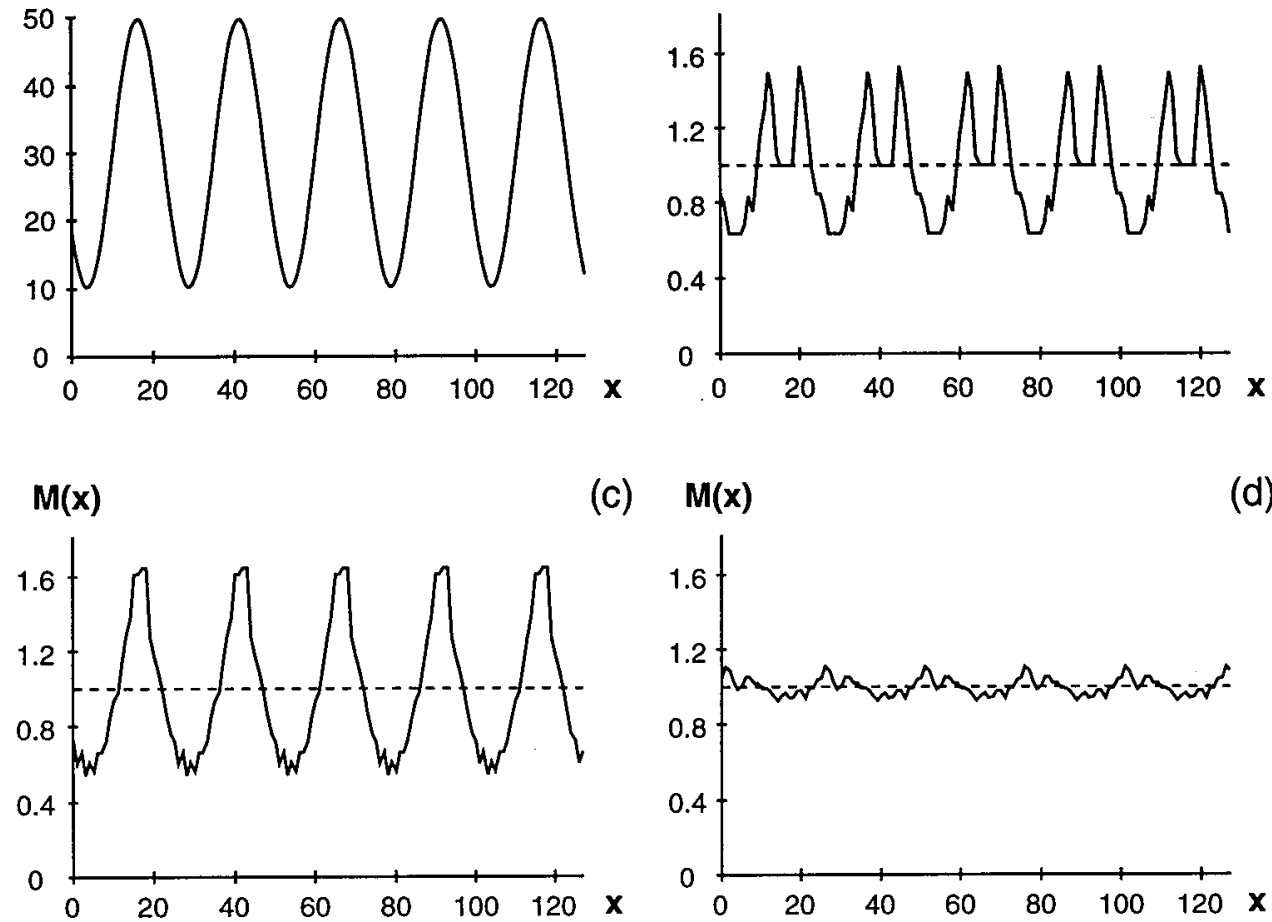

(c) $M(x)$

(d)

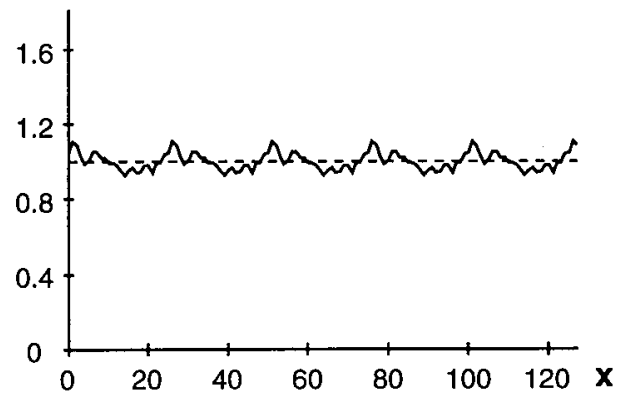

\section{Out(x)}

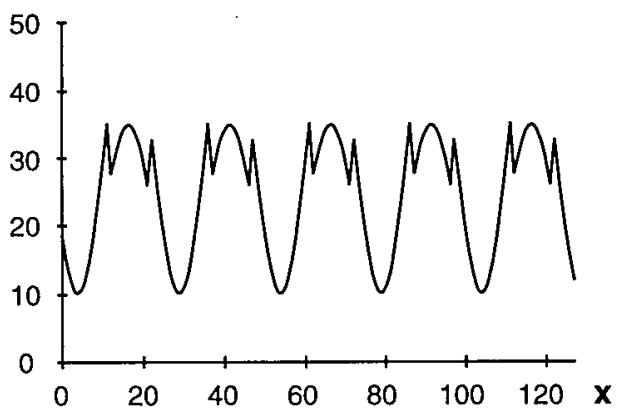

Figure 4.3. Response of the LDS algorithm to a harmonic signal (a), presented for $c=0.005$ (b), $c$ $=0.002(\mathrm{c})$ and $c=0.0008$ (d). Overall output of the algorithm for speckle reduction (e) is based on the LDS output of $(\mathrm{c})$ with $p_{s}=0.7$.

In the second processing step, a threshold is applied to the LDS output. All sample values of the input signal for which the corresponding LDS output exceeds 1 
are multiplied by a constant $p_{s}$ smaller than 1 . The effect of this operation is illustrated in figure $4.3-\mathrm{e}$, in which the overall output is shown of the speckle reduction algorithm, based on the correct LDS output of figure 4.3-c and $p_{s}$ set to 0.7 . The processed "speckle pattern" exhibits a signal related distortion, which increases the bandwidth of the amplitude signal, and, as will be shown in section 4.5, which combines a decrease in average speckle cell size with an corresponding increase in density of independent points. At the same time, the ratio $\sigma_{A} / \bar{A}$ is slightly influenced. If the two-dimensional version of the algorithm is applied to ultrasonic envelope data, these properties are expected to cause speckled areas to have a finer distribution, whereby the reduced speckle size may give the image subjectively a more homogeneous appearance, although speckle contrast is only slightly influenced. To achieve objective reduction of speckle contrast, a low pass filter (e.g., a moving average filter) has to be applied afterwards. The increased density of linearly independent points makes low pass filtering more effective, yielding an extra decrease in speckle contrast in comparison with filtering of the original image, as will also be demonstrated in section 4.5. Finally, since the amount by which a speckle peak is decreased is proportional to the peak value itself, differences in mean brightness between different areas are preserved.

The two parameters that have to be supplied to adjust the algorithm are the factor $p_{s}$ and the LDS scaling constant $c$. The former determines the relative amount by which the speckle peaks are lowered, and can be adjusted experimentally. The latter, $c$, is used to influence the action of the LDS algorithm, as was illustrated in figure 4.3. An expression will be derived for the upper and lower boundaries between which $c$ should be chosen.

In the previous section, it was found that in case a one-dimensional input signal consists of two amplitude levels $A_{1}$ and $A_{2}\left(A_{1}<A_{2}\right)$, separated by a straight edge, the location farthest from the transition where the LDS output exceeds 1 is situated at $x_{S}=1 /\left(2 c A_{1}\right)$ sample points from the foot. Consequently, if a 1D speckle cell is approximated by a peak value $A_{2}$, a trough value $A_{1}$ and two straight flanks, the two peaks in the LDS output that are associated with both flanks will overlap for $x_{S}>$ $S_{f f} / 2, S_{f f}$ being the speckle cell size in sample points, measured from foot to foot. Since this overlap is necessary for the correct functioning of the speckle reduction algorithm, an upper boundary is found: $c<1 /\left(S_{f f} A_{1}\right)$ or, with $S_{f f}=2 S$ ( $S$ being the average speckle size): $c<1 /\left(2 S A_{1}\right)$. On the other hand, if $x_{s}$ becomes too large, peaks and dips in the Mach bands distribution may interfere. A practical lower boundary is: $x_{s}<S_{f f} \Rightarrow c>1 /\left(2 S_{f f} A_{1}\right)=1 /\left(4 S A_{1}\right)$. Similarly, for the two-dimensional case it is found that: 


$$
\frac{1}{16 S_{x} \cdot S_{y} \cdot A_{1}}<c<\frac{1}{4 S_{x} \cdot S_{y} \cdot A_{1}}
$$

where $S_{x}$ is the average lateral and $S_{y}$ the average axial speckle size. The speckle trough level $A_{1}$ is approximated by a fraction of the (local) mean amplitude level. Note that for a fixed value of $c, A_{1}$ is allowed to vary by a factor of 4 , which makes the algorithm rather robust and insensitive to the exact choice of $c$. Application of the algorithm to real and simulated data (as in figure 4.3) for various values of $c$ indicated that the boundaries derived are indeed good indicators for the choice of $c$.

\subsubsection{Edge enhancement}

Only a minor extension is needed to make the algorithm suitable for the enhancement of vessel wall / lumen interfaces in all directions: a counter has to be added to the LDS algorithm to count the number of times that each location $(x, y)$ is covered by a spread function. Let the result of this operation be stored in the $2 \mathrm{D}$ array $N(x, y)$. Then the algorithm for speckle reduction plus edge enhancement is described by:

1. Application of the extended LDS algorithm. Output consists of the arrays $M(x, y)$ and $N(x, y)$.

2. For each envelope point:

$$
\begin{array}{lll}
\text { if }(M(x, y)>1)\{ & & \\
\quad \begin{array}{ll}
\text { if }\left(N(x, y)<T_{N}\right) \\
\text { else }
\end{array} & \text { Out }(x, y)=p_{s} \cdot A(x, y) ; & \text { (local edge) } \\
\text { Out }(x, y)=p_{e} \cdot A(x, y) ; & \text { (global edge) } \\
\text { else } & \text { Out }(x, y)=A(x, y) ; & \text { (no edge) }
\end{array}
$$

( $T_{N}$ is a constant threshold, $p_{e} \geq 1$ determines the weight of edge enhancement)

The $N$ array contains the information needed to decide whether or not an edge is global, i.e., whether it marks a transition between vessel wall and lumen (in Chapter 5 , the concept of the $N$ distribution will be generalized and the exact relationship will be derived between the local $N$ level and the local mean brightness in the echo image). Because the backscattered power from blood particles is low, the lumen is imaged as a dark area. Consequently, all points within the lumen have very wide spread functions, which will often reach to beyond the border of the lumen. 
A(y)

(a)

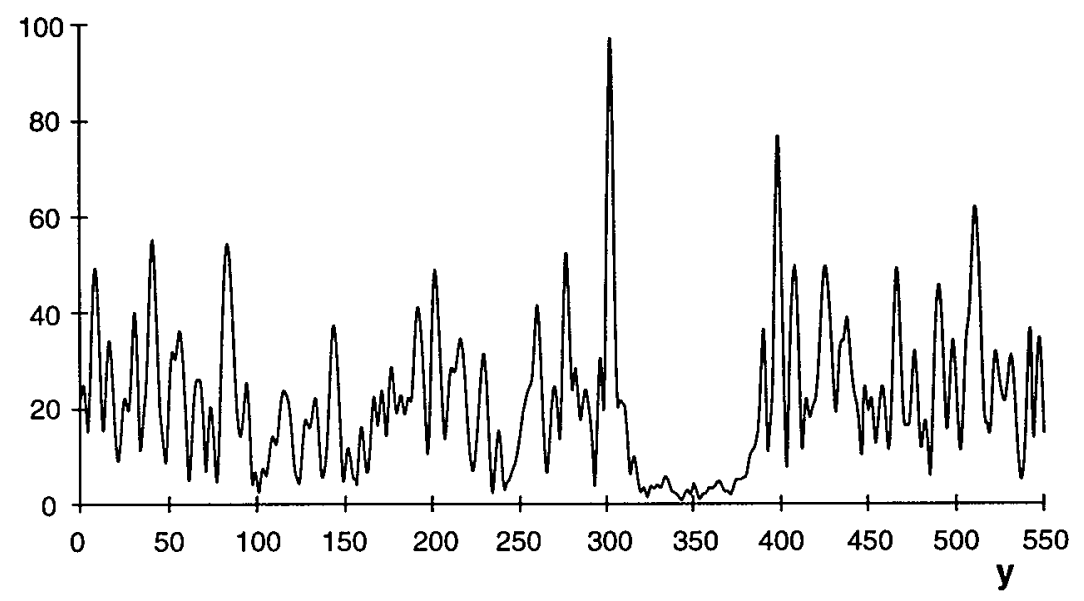

$M(y)$

(b)

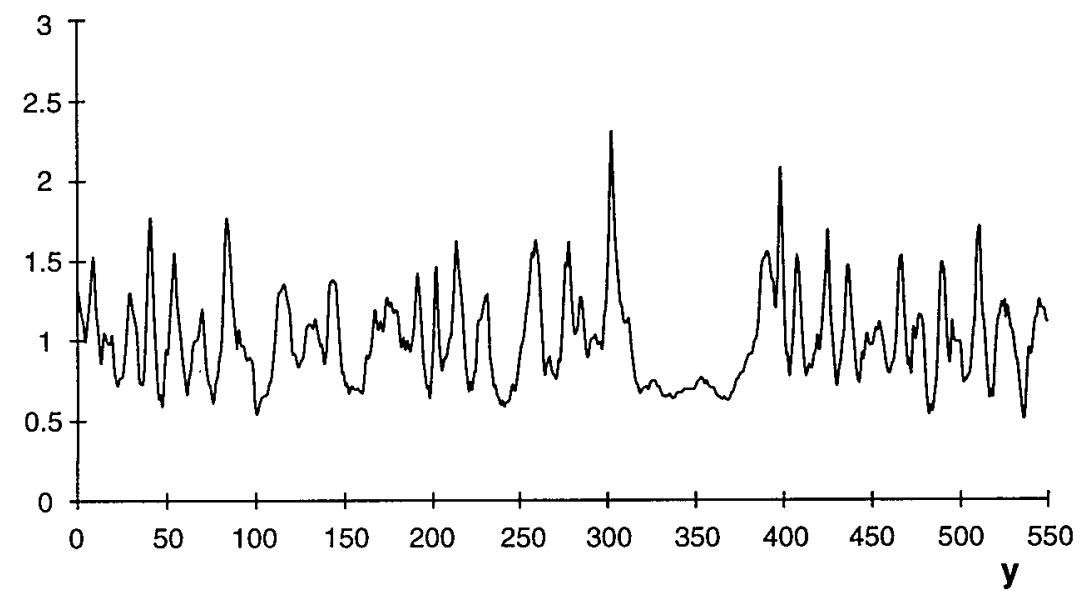

Figure 4.4. (a) Envelope signal with a liquid filled tube located between sample points 300 and 400 (corresponding to a width of $0.6 \mathrm{~cm}$ ), surrounded by speckle. (b) $M$ output of the extended LDS algorithm in response to the envelope signal of (a). Parameter $c=0.008$. (c) $N$ output of the extended LDS algorithm in response to the envelope signal of (a). (d) Processed envelope signal based on the $M$ and $N$ outputs of figures (b) and (c), respectively, with $p_{S}=0.6$ and $T_{N}=$ $1.6 \bar{N}_{\text {speckle }}=35$.

That is why $N(x, y)$ will have high values for envelope points near global edges and only moderate ones at local edges (at speckle granules). The threshold $T_{N}$ needs to be adjusted so that those high values are distinguished from the others. In practice, $T_{N}$ $=p_{N} \cdot \bar{N}_{\text {speckle }}$ appeared to work well $\left(\bar{N}_{\text {speckle }}=\right.$ the mean $N$ value of a speckled region, $\left.1<p_{N}<3\right)$. 
$N(y)$

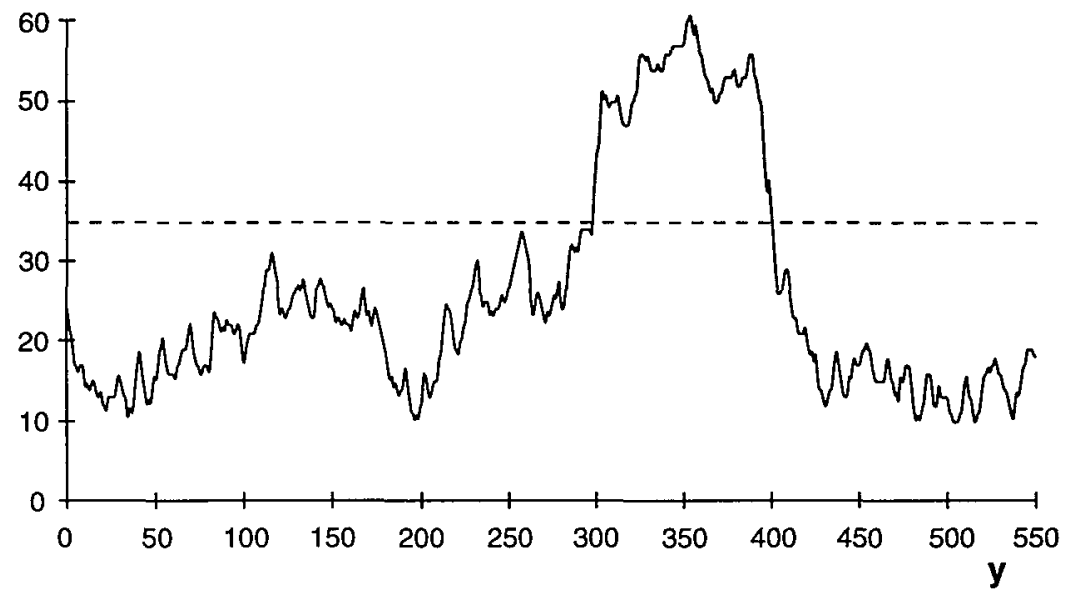

Out(y)

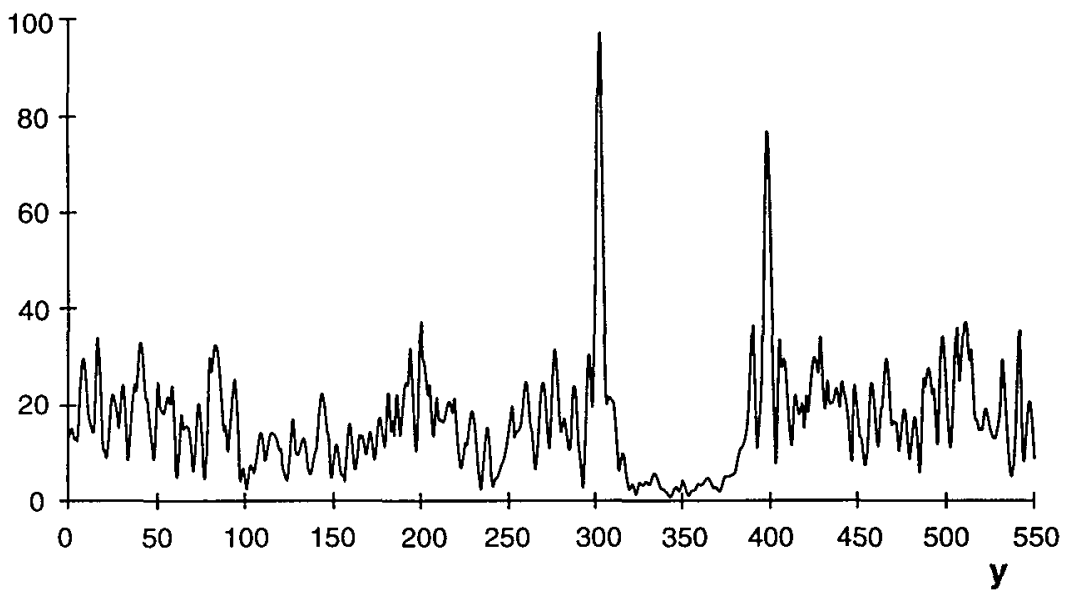

Figure 4.4. - continued

Evidently, step 2 is exactly similar to step 2 of the original algorithm, except for the case that a global edge is recognized: in that situation the corresponding envelope points are not lowered but remain unaffected $\left(p_{e}=1\right)$ or they are emphasized $\left(p_{e}\right.$ $>1$ ). Strictly speaking, for $p_{e}=1$ global edges are not enhanced, but since their amplitudes are preserved while the mean level of surrounding tissue is decreased, this will have the same effect. Figure 4.4-a shows the envelope of an rf line originating from a tissue mimicking phantom, enclosing a liquid filled tube modeling a blood vessel. The anterior wall of the tube is located somewhere at sample point 300 (corresponding to a depth of $4.8 \mathrm{~cm}$ ), the posterior wall at about sample point 400 (5.4 
$\mathrm{cm})$. Outside the tube, a typical speckle pattern is visible. The two output arrays of the extended LDS algorithm, $M(y)$ and $N(y)$, are shown in figures 4.4-b and -c, respectively. Clearly, the $N$ values in and around the lumen are high, allowing the recognition of the borders of the lumen as global edges. Figure 4.4-d presents the processed envelope, exhibiting reduced speckle and enhanced tube walls. The envelope pattern within the lumen has not been changed by the algorithm. But as the amplitude level is very low here, possible fluctuations would not have had any visible effect. The parameter values used are $c=0.008, T_{N}=1.6 \bar{N}_{\text {speckle }}=35, p_{s}=0.6$ and $p_{e}=1$.

One might wish to influence global edge enhancement by increasing the width of the region of high $N$ values just outside the lumen. This effect is achieved by making the spread functions extra wide for low amplitudes, which can be done by making the surface of the spread functions proportional to $1 / A^{q}(q>1)$ instead of $1 / A$. Cornsweet and Yellot Jr. (1985) proved that all theory associated with level-dependent spatial summation, including the production of Mach bands, still holds for $q>$ 1. The only difference is that $A$ has to be substituted by $A^{q}$ in all formulas. Also in case the lumen appears to be corrupted by noise, e.g., due to reverberations, it may be useful to choose $q>1$, so that the decreased number of low amplitude points inside the lumen is still high enough to produce high $N$ values just outside the lumen.

Speckle reduction and edge enhancement are further illustrated in figure 4.5. Figure 4.5-a shows a B-mode echo image that was taken from a tissue mimicking phantom enclosing a number of objects (in fact, the envelope line used in the example of figure 4.4 originated from this data set). In pictures $4.5-\mathrm{b}$ and $-\mathrm{c}$, the twodimensional $M$ and $N$ distributions are encoded in gray levels. Finally, picture 4.5-d presents the echo image after processing by the extended algorithm. Note that the borders of the tubes are enhanced in all directions. Parameters: $c=0.0015, d_{x} / d_{y}=$ $0.13, T_{N}=1.5 \bar{N}_{\text {speckle }}=181, p_{s}=0.5$ and $p_{\theta}=1.5$. Depending on viewing distance, the modified speckle texture may be perceived as exhibiting reduced speckle contrast. In addition, in clinical applications, edge enhancement might lead to an improved

Figure 4.5. (a) Image $\left(8 \times 8 \mathrm{~cm}^{2}\right)$ of a tissue mimicking phantom enclosing three liquid filled tubes (black), a number of spikes (white) and two objects having higher reflectivities (at the upper right). (b) $M$ output of the extended LDS algorithm in response to the amplitude distribution comprising the image of (a). Parameters: $c=0.0015, d_{x} / d_{y}=0.13$. (c) $N$ output of the extended LDS algorithm in response to the amplitude distribution comprising the image of (a). (d) Processed image based on the $M$ and $N$ outputs of figures (b) and (c), respectively, with $p_{S}=0.5$ and $T_{N}=1.5 \bar{N}_{\text {speckle }}=181$. (e) Image processed similarly to figure (d), except that $c=0.0021$. (f) Image processed similarly to figure (d), except that $d_{x} / d_{y}=0.2$. 


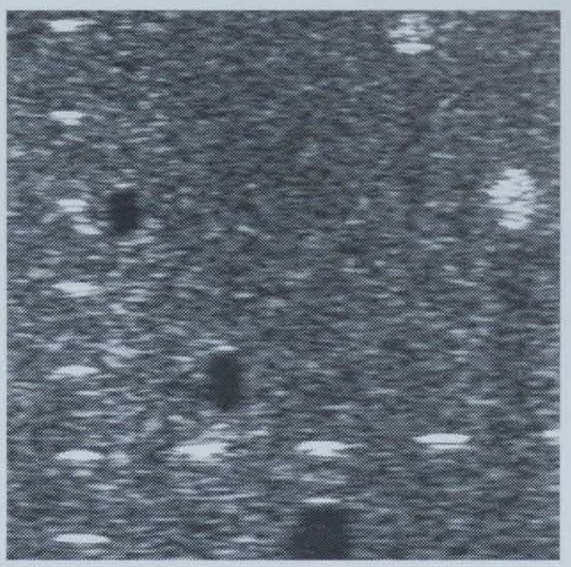

(a)

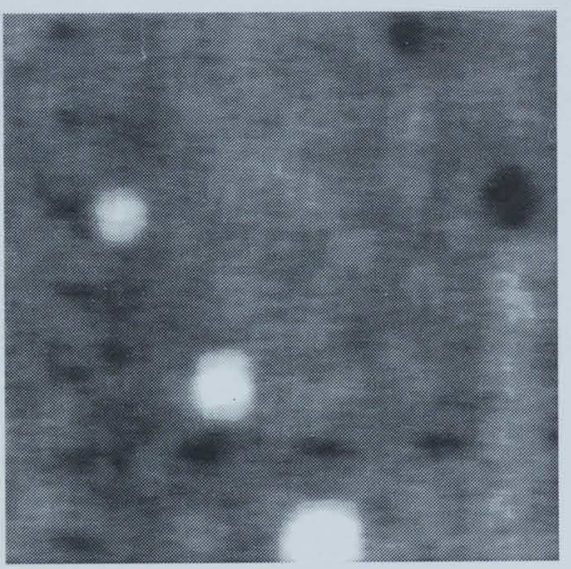

(c)

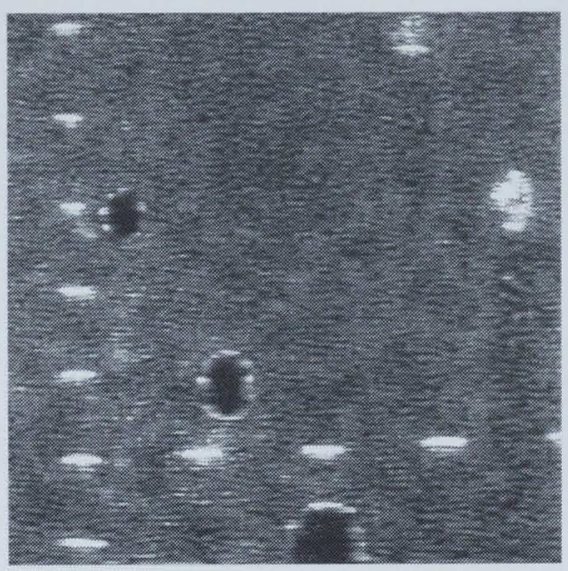

(e)

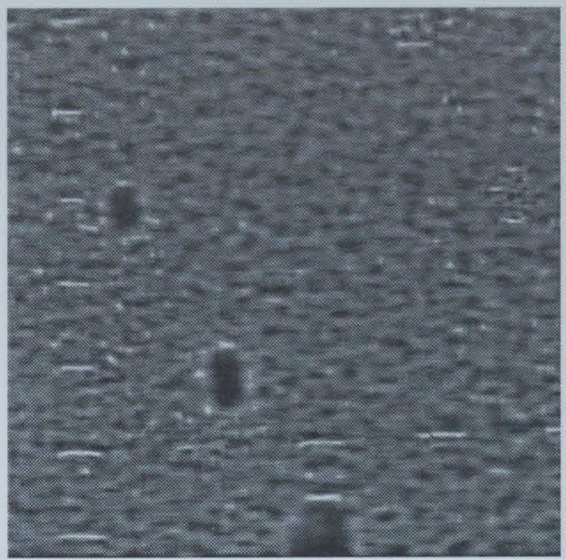

(b)

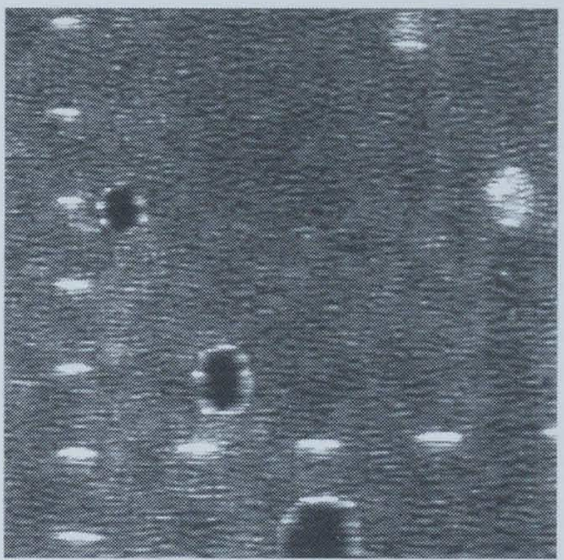

(d)

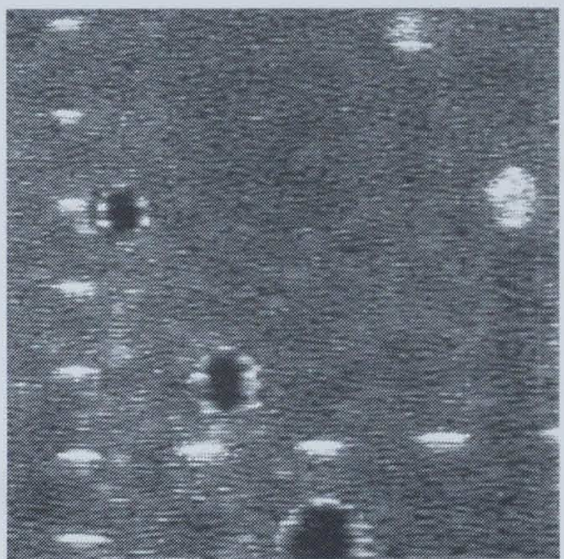

(f) 
visibility of small vessels that lack a clear definition in the original image, such as liver veins.

\subsubsection{Contrast enhancement}

As can be seen in figure 4.5-c, the $N$ distribution constitutes a measure of the local mean brightness in the B-mode image. Relatively light regions produce low $N$ values and vice versa. If a hypothetical uniform area of infinite extent has a constant amplitude level $A$, all points will produce the same spread function with base area $O=1 /(c A)$. This implies that every point will be exactly covered $O$ times by a spread function: $N(x, y)=O$ for every point $(x, y)$. If, however, a wide variety of amplitudes is found, as in speckled areas, an effective amplitude $A_{\text {eff }}$ may be defined as:

$$
A_{\text {eff }}(x, y) \stackrel{\text { def }}{=} \frac{1}{c \cdot N(x, y)}
$$

Thus, $A_{\text {eff }}(x, y)$ equals the amplitude of a homogeneous area that would produce exactly the same $N$ value at point $(x, y)$. Since many neighboring points will contribute to the value of $A_{\text {eff }}(x, y)$, it tends to vary only slowly in space (an assertion that is supported by the theory of Chapter 5 ). This provides a tool for increasing contrast between regions having different mean brightnesses. If every amplitude is substituted by a new one according to:

$$
A_{\text {new }}(x, y)=A_{\text {old }}(x, y) \cdot\left(1-p_{c} \cdot \frac{A_{\text {eff, min }}}{A_{\text {eff }}(x, y)}\right)
$$

with $0<p_{c}<1$ and $A_{\text {eff,min }}$ the minimum value of $A_{\text {eff }}(x, y)$, the contrast will be influenced. Suppose two neighboring regions can be distinguished in a $2 \mathrm{D}$ envelope data set, one globally having effective amplitude $A_{\text {eff, } 1}$ and the other $A_{\text {eff,2. Let }} A_{\text {eff, } 1}>$ $A_{\text {eff }, 2}$, then processing the amplitude values in regions 1 and 2 will result in: $A_{\text {new }}(x, y)=\eta_{1} \cdot A_{\text {old }}(x, y)$ for region 1 and $A_{\text {new }}(x, y)=\eta_{2} \cdot A_{\text {old }}(x, y)$ for region 2 with $0<$ $\eta_{2}<\eta_{1}<1$. (Note that $\eta_{2}<\eta_{1}$ is globally valid. However, fluctuations in $A_{e f f}(x, y)$ might incidentally cause $\eta_{2}>\eta_{1}$, especially near the transition between regions 1 and 2) Let $A_{1}$ and $A_{2}$ be the original amplitudes of two points located in regions 1 and 2, respectively. Mutual contrast is defined as:

$$
C_{1-2, \text { old }} \stackrel{\text { def }}{=} \frac{\left|A_{1}-A_{2}\right|}{A_{1}+A_{2}}
$$


In the new situation, it is found that:

$$
C_{1-2, \text { new }}=\frac{\left|\eta_{1} A_{1}-\eta_{2} A_{2}\right|}{\eta_{1} A_{1}+\eta_{2} A_{2}}=\frac{\left|A_{1}-A_{2}+\left(1-\frac{\eta_{2}}{\eta_{1}}\right) A_{2}\right|}{A_{1}+A_{2}-\left(1-\frac{\eta_{2}}{\eta_{1}}\right) A_{2}}>C_{1-2, \text { old }}
$$

The processing scheme as proposed preserves the larger-than-smaller-than order of global region brightness and, most importantly, it does not dramatically increase speckle contrast, since all points in one region are multiplied by approximately the same value. The resulting increase in the dynamics of the amplitude distribution can be compensated for by appropriate adjustment of the compression curve applied.

Contrast enhancement may be incorporated in processing step 2 of the algorithm by the expression:

$$
\begin{aligned}
& \text { if }(M(x, y)>1)\{ \\
& \text { if }\left(N(x, y)<T_{N}\right) \text { Out }(x, y)=p_{s} \cdot A(x, y) \cdot\left(1-p_{c} \cdot \frac{N(x, y)}{N_{\max }}\right) ; \quad \text { (local edge) } \\
& \text { else } \quad \operatorname{Out}(x, y)=p_{e} \cdot A(x, y) ; \quad \text { (global edge) } \\
& \text { \} } \\
& \operatorname{Out}(x, y)=A(x, y) \cdot\left(1-p_{c} \cdot \frac{N(x, y)}{N_{\max }}\right) ; \quad \text { (no edge) }
\end{aligned}
$$

Again, parameter $p_{s}$ determines the relative amount by which speckle peaks are lowered, and $p_{e}$ determines the weight of edge enhancement. Contrast enhancement is achieved by the factor $\left(1-p_{c} \cdot N(x, y) / N_{\max }\right)=\left(1-p_{c} \cdot A_{\text {eff, } \min } / A_{\text {eff }}(x, y)\right)$. The contrast enhancing factor is omitted at global edges because the $N$ values will be high there (on which the recognition of global edges is based) and will thus counteract the enhancement.

\subsection{Level of improvement}

Speckle reduction is conventionally described as a reduction of speckle contrast, which is equivalent to an increase in the point signal-to-noise ratio:

$$
\mathrm{SNR}_{\mathrm{A}}=\frac{\overrightarrow{\mathrm{A}}}{\sigma_{\mathrm{A}}}
$$


However, this measure does not take into account changes in speckle size and frequency, nor does it provide information about lesion detectability. In this respect, the lesion signal-to-noise ratio (Smith et al., 1983; Verhoeven and Thijssen, 1993) is more suitable. It describes the detectability of a lesion against a homogeneous background, and is defined as:

$$
\operatorname{SNR}_{L}=\frac{\left|\bar{A}_{2}-\bar{A}_{1}\right|}{\sqrt{\operatorname{var}\left(A_{1}\right)+\operatorname{var}\left(A_{2}\right)}} \cdot \sqrt{N L}
$$

where $\bar{A}_{1}$ and $\bar{A}_{2}$ are the mean lesion and background amplitude levels, $\operatorname{var}\left(A_{1}\right)$ and $\operatorname{var}\left(A_{2}\right)$ are the respective variances and NL equals the number of independent points in the lesion. $N L$ is approximately equal to the lesion area divided by the product $S_{x} \cdot S_{y}$. Lesion contrast is calculated as:

$$
C_{L}=\frac{\left|\bar{A}_{1}-\bar{A}_{2}\right|}{\bar{A}_{1}+\bar{A}_{2}}
$$

The present algorithm for speckle reduction mainly modifies the speckle size, while hardly influencing $S N R_{A}$ and $C_{L}$. This has the effect of an increase of the number of linearly independent points in the image. Figure 4.6-a shows a B-mode echo image of a homogeneous scattering medium. The speckle pattern is described by: $S_{x}=2.5, S_{y}=5.4$ and $S N R_{A}=1.87$. The image of figure $4.6-\mathrm{b}$ was produced by processing the original envelope signals with $d_{x} / d_{y}=0.5$ and with $p_{s}=0.5$. As a result, the speckle size decreased to $S_{x}=1.4$ and $S_{y}=3.5$ while $S N R_{A}$ remained at 1.93 . Increased density of independent points can be demonstrated by applying a moving average filter to the two images. Smoothing with a 5 points lateral by 10 points axial window results in a $S N R_{A}$ of 3.95 for the original image, and in a $S N R_{A}$ of 6.77 for the processed image. Since the moving average filter increases the $S N R_{A}$ proportionally to the square root of the number of independent points covered by the filter window, it follows that the effective number of points in the processed image is considerably higher, in accordance with the decreased average speckle cell size.

Improvement of lesion detectability can be demonstrated on the basis of the simulated image of figure $4.7-\mathrm{a}$, which was obtained by convolving a two-dimensional particle distribution with a $2 \mathrm{D}$ rf point spread function (Bamber and Dickinson, 1980). The image shows a central, circular, hypoechoic lesion with a diameter of $2 \mathrm{~cm}$ in a homogeneous background of $4.7 \times 5.4 \mathrm{~cm}^{2}$. Lesion scatter strength is $3 \mathrm{~dB}$ below background scatter strength. Particle density equals 26 per resolution cell. The number of parallel envelope lines is 113 , the number of sample 


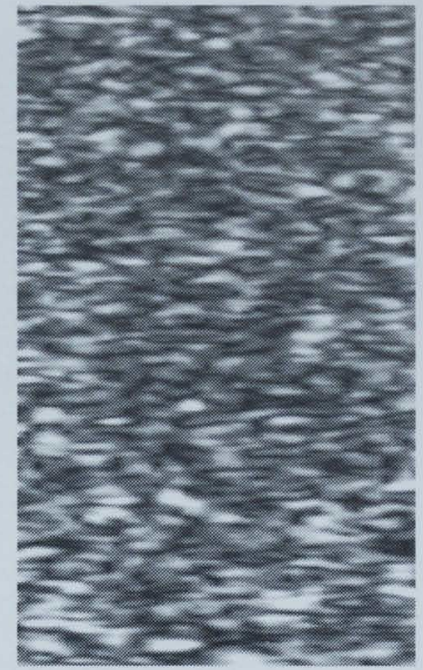

(a)

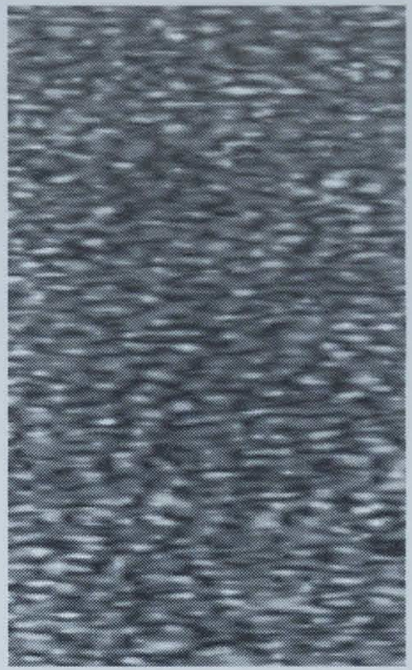

(b)

Figure 4.6. (a) Echo image of a homogeneous scattering medium. (b) Image after application of speckle reduction.

points per envelope line is 1024. Other parameters are: $S_{x}=3.6, S_{y}=7.7, N L=413$, $C_{L}=0.18, S N R_{A}=1.79$ (calculated over the whole image) and $S N R_{L}=0.46 \sqrt{N L}=9.4$. The envelope signals were processed with $d_{x} / d_{y}=0.5$ and $p_{s}=0.5$ (no edge enhancement and no contrast enhancement). As a result, $N L$ increased to 919 , while $C_{L}$ remained at $0.18 . S N R_{A}$ equaled 2.03. The lesion signal-to-noise ratio increased to $S N R_{L}=0.50 \sqrt{N L}=15.3$, which is about 63 percent higher than the original value. Successive application of speckle reduction to 10 independent realizations of the same lesion resulted in an average increase of the $S N R_{L}$ of 67 percent with a standard deviation of 5 percent.

In addition to speckle reduction, region contrast enhancement also improves the $S N R_{L}$. The original lesion signals of figure 4.7 -a were processed with $d_{x} / d_{y}=0.1$, $p_{c}=0.6$ and $q=2$ (no speckle reduction and no edge enhancement). Figure 4.7-b shows, by way of intermediate result, the $N$ distribution on an inverted gray scale. The mean lesion $N$ level equals 488 , the mean background $N$ level 303 . The processed image, shown in figure 4.7-c, has a lesion contrast $C_{L}$ of $0.33, N L$ equals 406 and $S N R_{L}=0.78 \sqrt{N L}=15.7$, corresponding to an increase of about 67 percent. $S N R_{A}$ decreased to 1.69 , which can be explained by the increase of $C_{L}$. Successive application of contrast enhancement to 10 independent realizations of the same lesion resulted in an average increase of the $S N R_{L}$ of 59 percent with a standard deviation of 6 


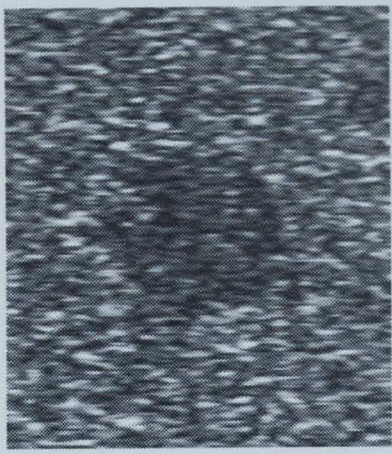

(a)

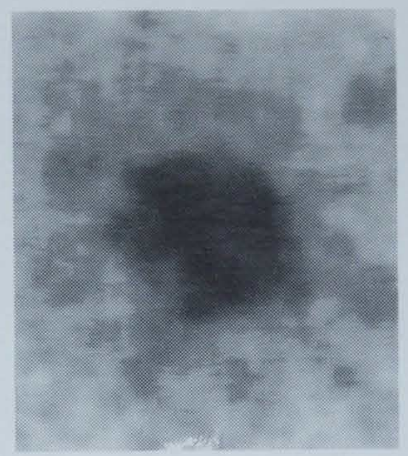

(b)

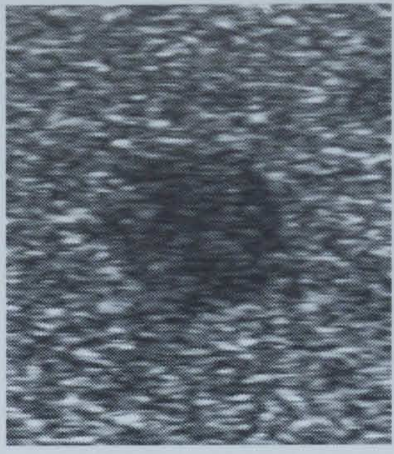

(c)

Figure 4.7. (a) Simulated echo image with a central, circular, hypoechoic lesion. (b) $N$ distribution on inverted gray scale. (c) Image after application of contrast enhancement.

percent.

Concerning edge enhancement, it is noted that edges are recognized on the basis of peaks in the Mach bands distribution, coinciding with above average values in the $N$ distribution. The Mach bands occur everywhere in the image, while the high $N$ values are found by applying a threshold $T_{N}$ to the $N$ distribution. Relative high values of $T_{N}$ are save, but they only allow edges of highly contrasting structures to be enhanced, especially vessel wall / lumen interfaces. For lower values of $T_{N}$, also edges of less contrasting objects are recognized. However, due to the variance in the $N$ distribution (an expression of which is derived in Chapter 5), false enhancement at speckle peaks become more likely.

\subsection{Discussion}

\section{- computational cost}

The total number of basic operations involved in the enhancement of an echo image depends on the image content. Large dark areas produce wide spread functions giving increased processing time while strong reflectors have the opposite effect. In addition, computational cost is strongly influenced by the lateral and axial sampling frequencies that are applied. A doubling of the axial sampling frequency $f_{s, A}$ leads to a doubling of the number of amplitude points in the axial direction as well as a doubling of the base areas of all spread functions, which results in a qua- 
drupling of the number of operations required for the calculation of the Mach bands distribution $M(x, y)$. That is why it is advised not to exceed by too much the lower limit of $f_{S, A}$, which equals three times the Nyquist sampling rate (Eq. (4.4)).

For a given amplitude distribution $A(x, y)$, the following operations have to be carried out for each point by the extended LDS algorithm:

- Calculation of the lateral and axial radius of support of the spread function $\left(d_{x}\right.$ and $d_{y}$ respectively). If $d_{x} / d_{y}=S_{x} / S_{y}$ and the amplitude level is $A$, then $d_{y}=$ $\sqrt{S_{y} /\left(4 c A S_{x}\right)}$ and $d_{x}=\left(S_{x} / S_{y}\right) \cdot d_{y}$. The number of points $O$ covered by the spread function equals $O=1 /(c A)=4 d_{x} d_{y}$.

- Adding the height of the spread function $(=c A)$ to $M(x, y)$ for all points $(x, y)$ covered.

- Adding 1 to $N(x, y)$ for all points $(x, y)$ covered.

If the values $d_{x}, d_{y}$ and $c A$ are stored in a look-up table for every possibly occurring amplitude value $A$, the extended action of the LDS algorithm is reduced to 20 summations for a sample point having amplitude $A$. In the processing step for image enhancement (Eq. (4.14)), a maximum of 1 division, 2 multiplications and 1 subtraction is needed for the processing of one amplitude point. Thus, as all operations are simple and can be easily and quickly executed by hardware, it is expected that the algorithm is suitable for real-time applications.

\section{- robustness}

The only two system dependent parameters that have to be supplied are the LDS scaling constant $c$ and the ratio $d_{x} / d_{y}$ of the lateral and axial radii of support. An expression was derived for the range of valid $c$-values (Eq. (4.8)), and it turned out that $c$ is allowed to vary by a factor of 4 between an upper and a lower boundary determined by the lateral and axial speckle cell sizes and the speckle trough amplitude level. Since these parameters will show some variation in space, it is advised to use average values and to choose $c$ from the middle of its valid range. At some locations in the image it may happen that the fixed value of $c$ is not appropriate, causing a speckle peak to be incompletely lowered or two neighboring peaks to be processed as one large peak. However, this seems to have only minor influence on the image quality. Figure 4.5-e demonstrates the effect of increasing $c$ by 40 percent. Apparently, the image is only slightly affected. Moreover, figure 4.5-f shows that increasing the ratio $d_{x} / d_{y}$ by 50 percent also has only a minor effect. These results 
suggest that the algorithm is rather insensitive to the exact choice of the two system parameters supplied. Its performance remains quite satisfactory over a fairly wide range of values, justifying the qualification "robust". Robustness of speckle reduction can further be increased by adapting $c$ to the local mean amplitude level, so that the algorithm is automatically adjusted according to the local image content. The parameters $p_{s}, p_{e}$ and $p_{c}$ are selected manually. Their adjustment is rather subjective and comparable to e.g. the monitor controls for "brightness" and "contrast".

\section{- flexibility}

According to the processing scheme proposed, the Mach bands distribution and the $N$ distribution are inextricably linked: by assigning concrete values to the scaling constant $c$ and the ratio $d_{x} / d_{y}$ in order to control the generation of Mach bands, the $N$ output is defined as well. However, the $N$ distribution may also be regarded as an independent process. By separating the calculations of $M(x, y)$ and $N(x, y)$, flexibility of the overall method is increased (at the expense of an approximate doubling of the computational cost). Individual parameter settings allow the characteristics of the $N$ distribution to be influenced without disturbing the Mach bands distribution. As a result, edge enhancement and contrast enhancement can be controlled independently from speckle reduction. The precise way in which $c, q$ and $d_{x} / d_{y}$ influence the local mean $N$ level, $\operatorname{var}(N)$ and the transient response, is analyzed in Chapter 5.

\section{- application in sector scanning systems}

The algorithm is based on the assumption that the speckle cell size is quite constant over the image, although, as was shown, variations of the order of 50 percent are not problematic. In sector scanning systems, the axial speckle cell size remains rather constant, while the speckle cell size in the lateral direction, expressed as the number of sample points, may exhibit large variations over depth, depending on the beam shape and the angle between consecutive scan lines. In that case, application of the LDS algorithm is restricted to the focal region. The one-dimensional version of the algorithm can still be used in the far field, but this has the disadvantage that edge enhancement in the lateral direction is not supported.

\subsection{Conclusion}

The application of the extended LDS algorithm to two-dimensional amplitude distributions yields useful information for the reduction of speckle, the enhance- 
ment of edges and the enhancement of contrast in the corresponding brightness images. These features, in combination with the algorithm's simplicity and robustness, make it a promising new approach to real-time ultrasonic image enhancement.

\section{REFERENCES}

Bamber J.C. and Daft C. (1986): Adaptive filtering for reduction of speckle in ultrasonic pulse-echo images, Ultrasonics 24, 41-44.

Bamber J.C. and Dickinson R.J. (1980): Ultrasonic B-scanning: a computer simulation, Phys. Med. Biol. 25, 463-479.

Cornsweet T.N. and Yellot Jr. J.I. (1985): Intensity-dependent spatial summation, J. Opt. Soc. Am. A 2, 1769-1786.

Crawford D.C., Cosgrove D.O., Tohno E., Svensson W.E., Al-Murrani B., Bell D.S., Stepniewska $\mathrm{K}$. and Bamber J.C. (1992): Visual impact of adaptive speckle reduction on US B-mode images, Radiology 183, 555-561.

Dickinson R.J. (1982): Reduction of speckle in ultrasound B-scans by digital processing, in Acoustical Imaging 12, Ash E.A. and Hill C.R., Eds., Plenum Press, New York, pp. 213-224.

Koo J.I. and Park S.B. (1991): Speckle reduction with edge preservation in medical ultrasonic images using a homogeneous region growing mean filter (HRGMF), Ultrasonic Imaging 13, 211-237.

Kotropoulos C. and Pitas I. (1992): Optimum nonlinear signal detection and estimation in the presence of ultrasonic speckle, Ultrasonic Imaging 14, 249-275.

Loupas T., McDicken W.N. and Allan P.L. (1989): An adaptive weighted median filter for speckle suppression in medical ultrasonic images, IEEE Trans. Circuits Systems 36, 129-135.

Reese G. (1992): Image enhancement by intensity-dependent spread functions, CVGIP-Graph. Mod. Im. 54, 45-55.

Smith S.W., Wagner R.F., Sandrik J.M. and Lopez H. (1983): Low contrast detectability and contrast/detail analysis in medical ultrasound, IEEE Trans. Sonics Ultrasonics 30, 164-173.

Verhoeven J.T.M. and Thijssen J.M. (1993): Improvement of lesion detectability by speckle reduction filtering: a quantitative study, Ultrasonic Imaging 15, 181-204.

Wagner R.F., Smith S.W., Sandrik J.M. and Lopez H. (1983): Statistics of speckle in ultrasound Bscans, IEEE Trans. Sonics Ultrasonics 30, 156-163. 


\section{5}

\section{Improved Lesion Detection by Level-Dependent Spatial Summation}

Chapter 5, with exception of figure 5.9, is based on the publication:

R.J. Collaris and A.P.G. Hoeks (1995): Improved lesion detection by level-dependent spatial summation, Ultrasonic Imaging 17, 197-226.

Figure 5.9 was taken from the paper:

R.J. Collaris and A.P.G. Hoeks (1995): Improved lesion detection by level-dependent spatial summation, Acoustical Imaging 22, 257-262. 
Chapter 5 


\section{List of main symbols}

$x, y \quad$ space coordinates. Variable $x$ denotes lateral position and variable $y$ denotes axial position.

$A(x, y)$ spatial amplitude distribution.

$N(x, y)$ output of the algorithm for level-dependent spatial summation.

$\mathrm{O}(\mathrm{x}, \mathrm{y})$ area covered by the window produced by amplitude point $A(x, y)$ : $O(x, y)=4 d_{x}(x, y) \cdot d_{y}(x, y)$.

$\mathrm{d}_{\mathrm{x}}(\mathrm{x}, \mathrm{y})$ lateral radius of support of the window produced by point $A(x, y)$. The window length in the lateral direction equals $2 d_{x}(x, y)$ sample points.

$d_{y}(x, y)$ axial radius of support of the window produced by point $A(x, y)$. The window length in the axial direction equals $2 d_{y}(x, y)$ sample points.

c scaling constant determining the mean window area $\bar{O}$.

q nonzero power to which all amplitude points are raised in the process of level-dependent spatial summation.

$k$ ratio of the lateral and axial radii of support: $d_{x}(x, y)=k \cdot d_{y}(x, y)$.

$\mathrm{S}$ mean speckle size, measured as the area under the normalized autocovariance function of the amplitude distribution $A$.

$L_{N} \quad$ correlation cell size as experienced by the algorithm for level-dependent spatial summation.

$L_{A} \quad$ correlation cell size as experienced by the $L_{2}$-mean filter. $L_{A}$ closely approximates the speckle size $S$.

\subsection{Abstract}

A novel signal postprocessing method has been developed for application in lesion detection. A signal distribution, directly related to the two-dimensional envelope distribution comprising a B-mode image, is convolved with a block shaped kernel of which both base area (i.e., window size) and height are modulated by the local signal level. A theoretical analysis shows that the output includes information regarding the local mean brightness in the original image. This information can be displayed as a gray scale image. The predicted behavior agrees with experimental observations. On the basis of twenty simulated lesion images, it is demonstrated that the present algorithm is superior to the conventional $\mathrm{L}_{2}$-mean filter in lesion detection problems, whereby the performance is quantified by the area $A_{R O C}$ under the Receiver Operating Characteristic. An improvement in the median value of $A_{R O C}$ of the order of 0.1 was observed. Two parameters are used for adjustment. The total number of summations involved in the processing of a frame is comparable to the number required by the arithmetic mean filter, allowing real-time implementa- 
tion.

Key words: Image processing; lesion detection; level-dependent spatial summation; Receiver Operating Characteristic; signal processing; ultrasound.

\subsection{Introduction}

The detection of focal lesions is of utmost importance in medical diagnosis. In ultrasonic imaging, however, the perception of subtle differences in local mean gray level is impeded by the grainy character of the images. The speckle pattern exhibited is generally viewed as a form of acoustic noise, resulting from interference of ultrasound echoes coming from tiny structures too close to be resolved. Various signal postprocessing techniques have been described to increase the detectability of slightly contrasting structures in the image. Frequency compounding (Magnin et al., 1982; Melton Jr. and Magnin, 1984; Trahey et al., 1986), temporal frame averaging (Bartrum Jr. and Crow, 1980) and spatial low pass filtering (Bamber and Daft, 1986; Dickinson, 1982; Koo and Park, 1991; Kotropoulos and Pitas, 1992; Loupas et al., 1989; Verhoeven and Thijssen, 1993) of the images are established methods exchanging image resolution, in space or in time, for an improved point signal-tonoise ratio. Gray level transforms too were shown to influence lesion detectability (Thijssen et al., 1988). Concerning spatial filtering, it is noted that the processed image is generally obtained by sliding a fixed size window over the original image. For each pixel in the original image, a new value is calculated, based on the pixel value itself and the gray level distribution in its local neighborhood. Three types of these conventional filters have been investigated by Kotropoulos and Pitas (1992) as well as Verhoeven and Thijssen (1993): the arithmetic mean filter, the median filter and the $\mathrm{L}_{2}$-mean filter. As a measure of performance in lesion detection, they used the area under the Receiver Operating Characteristic (ROC curve) (Green and Swets, 1988; Swets and Pickett, 1982; Wagner, 1986) or the lesion signal-to-noise ratio (Smith et al., 1983; Thijssen et al., 1988; Verhoeven and Thijssen, 1993; Wagner and Brown, 1985). Both authors observed that the $\mathrm{L}_{2}$-mean filter has the best performance. This filter replaces each point in the original signal distribution by the square root of the average squared signal value in the neighborhood (Pitas and Venetsanopoulos, 1986).

In the present paper, a signal postprocessing technique is proposed which differs significantly from the filters mentioned above. The concept of level-dependent spatial summation (Collaris and Hoeks, 1994) is applied to the 2D envelope distribution comprising an ultrasound B-mode image; unlike conventional filters, the dis- 
tribution is convolved with a block shaped kernel having a base area and a height that are not fixed, but vary with the local (instantaneous) signal level. The resulting distribution can be presented as a gray level image. A formal description of the process is given as well as a theoretical analysis of the output (in Collaris and Hoeks (1994) referred to as the $N$ distribution). It is demonstrated that the output includes information on the local mean signal level. Experimental results are reported, confirming the theoretical analysis. Finally, an ROC analysis is given, showing that the present data processing scheme is superior to the $\mathrm{L}_{2}$-estimator in lesion detection problems.

\subsection{Method}

The algorithm for level-dependent spatial summation is applied to the two-dimensional amplitude distribution comprising a B-mode echo image. Each amplitude point $A(x, y)$ is raised to some nonzero power $q$ and multiplied by a positive scaling constant $c$. Coordinates $x$ and $y$ denote lateral and axial positions, respectively. The output $N(x, y)$ is obtained by convolving the resulting distribution $c A^{q}(x, y)$ with a level-dependent kernel $w_{0}$ according to:

$$
N(x, y)=\sum_{x^{\prime}} \sum_{y^{\prime}} c A^{q}\left(x^{\prime}, y^{\prime}\right) \cdot w_{0}\left(\left(x-x^{\prime}, y-y^{\prime}\right) ; c A^{q}\left(x^{\prime}, y^{\prime}\right)\right)
$$

Kernel $w_{0}$ is defined by:

$$
w_{0}\left((x, y) ; c A^{q}\right)=\left\{\begin{array}{cl}
\frac{1}{c A^{q}} & \text { for }|x| \leq d_{x} \text { and }|y| \leq d_{y} \\
0 & \text { otherwise }
\end{array}\right.
$$

where

$$
d_{x}=\sqrt{\frac{k c A^{q}}{4}}
$$

is the lateral radius of support and

$$
d_{y}=\sqrt{\frac{c A^{q}}{4 k}}
$$


is the axial radius of support. The factor $k=d_{x} / d_{y}$ defines the ratio between the lateral and axial radii of support (i.e., the ratio between the lateral and axial dimensions of the kernel $\left.w_{0}\right)$. The rectangular area $O$ covered by $w_{O}$ is dependent on the local signal level and equals $O=4 d_{x} \cdot d_{y}=c A^{q}$. The volume enclosed by $w_{0}, O /\left(c A^{q}\right)$, does not depend on the local signal level and is always equal to one. The convolution described by Eq. (5.1) can be interpreted as follows. Each amplitude point $A(x, y)$ produces a rectangular window, centered around point $(x, y)$. The area $O(x, y)$ covered by the window is proportional to $A^{q}(x, y)$, while the ratio $k=d_{x}(x, y) / d_{y}(x, y)$ of the lateral and axial dimensions of the window remains fixed. Afterwards, the $N$ distribution includes the number of times each location was covered by a window. Note that although the area $O(x, y)$ is a continuous function of $A(x, y)$, the number of points covered by the window is always an integer. For a positive exponent $q$, high amplitude values produce large window areas while low amplitudes produce small ones. Consequently, high $N$ values indicate bright regions in the echo image while low $N$ values correspond to dark regions. For a negative $q$, the opposite is true.

Note: The $N$ distribution was first introduced in Chapter 4 as a supplementary product of the Mach bands algorithm. In the present Chapter, however, the $N$ distribution is regarded as an independent process, whereby $O$ is equal to $C A^{q}$. In contrast, as a consequence of the way in which the Mach bands algorithm was defined, $O$ equaled $1 /\left(C A^{q}\right)$ in Chapter 4 , which implies that the parameters $c$ and $q$ in the present chapter correspond to $1 / c$ and $-q$ in the previous chapter, respectively. Moreover, in Chapter 4 exponent $q$ was restricted to $q>0$, while in Chapter $5, q$ is allowed to be positive as well as negative, by which the concept of the $N$ distribution is generalized.

\subsection{Theoretical analysis}

The $N$ distribution is first investigated in response to a stationary amplitude distribution. Thereafter, attention is paid to the transient response.

\subsubsection{Response to a stationary amplitude signal}

For notational convenience, the analysis is restricted to one dimension. The derivations for two dimensions are, however, completely analogous. It is assumed that the algorithm for level-dependent spatial summation is applied to a stationary amplitude distribution $A$ of infinite extent showing a fully developed speckle pattern. Each amplitude point $A(y)$ produces a window, centered around $y$, with length $O(y)$ $=2 d_{y}(y)=c A^{q}(y)$. Effort is directed to finding expressions for the mean $N$ and $O$ values and the variances in $N$ and $O$, and derived expressions. 


\section{- mean $O$, mean $N$}

As is shown in Appendix A, the mean $N$ value is closely approximated by the mean window area $\bar{O}$. That is:

$$
\bar{N} \approx \bar{O}=\overline{c A^{q}}=\int_{0}^{\infty} c A^{q} \cdot p(A) d A
$$

The probability density function $p(A)$ describes a Rayleigh distribution (Wagner et al., 1983):

$$
p(A)=\frac{A}{\psi} \cdot e^{-\frac{A^{2}}{2 \psi}} \quad(A \geq 0)
$$

where parameter $\psi$ depends on the mean-square scattering amplitude of the particles:

$$
\psi=\frac{\overline{\left(\mathrm{A}^{2}\right)}}{2}=\frac{2}{\pi}(\overline{\mathrm{A}})^{2}
$$

Combining Eq. (5.4) and Eq. (5.5) yields:

$$
\overline{\mathrm{N}} \approx \overline{\mathrm{O}}=\frac{\mathrm{c}}{\psi} \cdot \int_{0}^{\infty} A^{q+1} \cdot e^{-\frac{A^{2}}{2 \psi}} d A=c \cdot(2 \psi)^{\frac{q}{2}} \cdot \Gamma\left(\frac{q}{2}+1\right)
$$

where $\Gamma$ indicates the gamma function. Eq. (5.7) is only true for values of the exponent $q>-2$. For $q \leq-2$, the integral becomes infinite. Substituting Eq. (5.6) in Eq. (5.7), the expression for $\bar{N}$ becomes:

$$
\bar{N} \approx \bar{O}=c \bar{A}^{q} \cdot\left(\frac{2}{\sqrt{\pi}}\right)^{q} \cdot \Gamma\left(\frac{q}{2}+1\right)
$$

Thus, the mean $N$ value is proportional to the mean amplitude level raised to the power of $q$.

\section{- variance in 0}

The calculation of $\operatorname{var}(O)$ requires the expression for $\overline{O^{2}}$ : 


$$
\overline{O^{2}}=\overline{\left(c A^{q}\right)^{2}}=\int_{0}^{\infty}\left(c A^{q}\right)^{2} \cdot p(A) d A=c^{2} \cdot(2 \psi)^{q} \cdot \Gamma(q+1)
$$

Combining Eqs. (5.7) and (5.9) gives:

$$
\operatorname{var}(O)=\overline{O^{2}}-\bar{O}^{2}=c^{2} \cdot(2 \psi)^{q} \cdot\left\{\Gamma(q+1)-\Gamma^{2}\left(\frac{q}{2}+1\right)\right\} \quad(q>-1)
$$

\section{- variance in $N$}

Because of the stationarity assumed, $\operatorname{var}(N)$ is not a function of position. An estimation will be made of $\operatorname{var}(N)$ at $y=0$. The chance that the window of amplitude point $A(y)(y>0)$ reaches to $y=0$ is equal to the probability $p(O(y) \geq 2 y)$, which is calculated as:

$$
\begin{aligned}
& q>0: \quad p(O(y) \geq 2 y)=p\left(A(y) \geq\left(\frac{2 y}{c}\right)^{\frac{1}{q}}\right)=\int_{\left(\frac{2 y}{c}\right)^{\frac{1}{a}}}^{\infty} \frac{A}{\psi} \cdot e^{-\frac{A^{2}}{2 \psi}} d A=e^{-\frac{\left(\frac{2 y}{c}\right)^{\frac{2}{a}}}{2 \psi}} \\
& q<0: \quad p(O(y) \geq 2 y)=p\left(A(y) \leq\left(\frac{2 y}{c}\right)^{\frac{1}{q}}\right)=1-e^{-\frac{\left(\frac{2 y}{c}\right)^{\frac{2}{q}}}{2 \psi}}
\end{aligned}
$$

For each amplitude point, a Boolean stochastic variable $B$ is defined that is associated with the chance that the corresponding window covers the point $y=0$ :

$$
B(y)=\left\{\begin{array}{lll}
0 & \text { if } O(y)<2 y \Rightarrow p(B(y)=0)=p(O(y)<2 y) \\
1 & \text { if } \quad O(y) \geq 2 y \Rightarrow p(B(y)=1)=p(O(y) \geq 2 y)
\end{array}\right.
$$

Then, the $N$ value at $y=0$ equals $N(y=0)=\sum_{y=-\infty}^{\infty} B(y)$ and

$$
\bar{N}=\overline{N(y=0)}=1+2 \sum_{y=1}^{\infty} \overline{B(y)}
$$

In analogy to Smith et al. (1983), the concept of the correlation cell is employed to calculate $\operatorname{var}(N)$. It is assumed that an odd number of $L_{N}$ neighboring $B(y)$ 's $\left(L_{N}\right.$ 
will depend on the speckle size as well as on $q$ ) can be combined into a group such that the $B(y)$ 's within one group are fully correlated, while no correlation exists between different groups. Thus:

$$
N(y=0)=\sum_{n=-\infty}^{\infty} G(n) \quad \text { with } G(n)=\sum_{y=-\left(L_{N}-1\right) / 2}^{\left(L_{N}-1\right) / 2} B\left(n L_{N}+y\right)
$$

and

$$
\operatorname{var}(G(n)) \approx L_{N}^{2} \cdot \operatorname{var}\left(B\left(n L_{N}\right)\right)
$$

It follows that:

$$
\operatorname{var}(N) \approx \sum_{n=-\infty}^{\infty} \operatorname{var}(G(n))=2 \sum_{n=1}^{\infty} \operatorname{var}(G(n)) \approx 2 L_{N}^{2} \cdot \sum_{n=1}^{\infty} \operatorname{var}\left(B\left(n L_{N}\right)\right)
$$

An expression for $\operatorname{var}\left(B\left(n L_{N}\right)\right)$ is found by combining Eqs. (5.11) and (5.12):

$$
\operatorname{var}\left(B\left(y=n L_{N}\right)\right)=\overline{B^{2}\left(n L_{N}\right)}-\overline{B\left(n L_{N}\right)}{ }^{2}=e^{-\frac{\left(\frac{2 L_{N} \cdot n}{c}\right)^{\frac{2}{q}}}{2 \psi}}-e^{-\frac{\left(\frac{2 L_{N} \cdot n}{c}\right)^{\frac{2}{a}}}{\psi}}
$$

The expression for $\operatorname{var}(N)$ becomes:

$$
\operatorname{var}(N) \approx 2 L_{N}^{2} \cdot \sum_{n=1}^{\infty}\left(e^{-\frac{\left(2 L_{N} \cdot n / C\right)^{\frac{2}{9}}}{2 \psi}}-e^{-\frac{\left(2 L_{N} \cdot n / c\right)^{\frac{2}{9}}}{\psi}}\right)
$$

As is proven in Appendix $B$, it is eventually found that:

$$
\operatorname{var}(N) \approx L_{N} \cdot\left|1-2^{-q / 2}\right| \cdot \bar{N}
$$

Or with Eq. (5.8):

$$
\operatorname{var}(N) \approx L_{N} \cdot c \bar{A}^{q} \cdot \frac{\left|2^{q}-2^{q / 2}\right|}{\pi^{q / 2}} \cdot \Gamma\left(\frac{q}{2}+1\right)
$$

In the two-dimensional analogs of Eqs. (5.19) and (5.20), the variable $L_{N}$ is replaced by the product $L_{N, x} \cdot L_{N, y}$ of the lateral and axial correlation cell sizes. Table 5.1 
Table 5.1. Summary of Eqs. (5.8) and (5.19) for a number of exponents $q$.

\begin{tabular}{c|cccccc}
$\mathrm{q}$ & -1 & -0.5 & 0.5 & 1 & 2 & 5 \\
\hline$\overline{\mathrm{N}} /\left(\mathrm{c} \overline{\mathrm{A}}^{\mathrm{q}}\right)$ & 1.57 & 1.15 & 0.96 & 1.00 & 1.27 & 6.08 \\
\hline $\operatorname{var}(\mathrm{N}) /\left(\mathrm{L}_{N} \bar{N}\right)$ & 0.41 & 0.19 & 0.16 & 0.29 & 0.50 & 0.82
\end{tabular}

summarizes Eqs. (5.8) and (5.19) for a number of particular values of exponent $q$.

\section{- point signal-to-noise ratio}

The point signal-to-noise ratio of the $N$ distribution is defined as $S N R_{N} \stackrel{\text { def }}{=}$ $\bar{N} / \sigma_{N}$. Substituting $\bar{N} \approx \bar{N}^{2} / \bar{O}$ in Eq. (5.19) leads to:

$$
\mathrm{SNR}_{\mathrm{N}}=\frac{1}{\sqrt{\left|1-2^{-\mathrm{q} / 2}\right|}} \cdot \sqrt{\frac{\overline{\mathrm{O}}}{\mathrm{L}_{\mathrm{N}}}}
$$

Thus, the point signal-to-noise ratio is proportional to the square root of the average number of independent correlation cells covered by a window with size $\bar{O}$. The proportionality factor is a function of the exponent q. Obviously, Eq. (5.21) has the same structure as the point signal-to-noise ratio of the amplitude distribution smoothed by an arithmetic mean filter with window size $\bar{O}$ (Smith et al., 1983):

$$
\mathrm{SNR}_{\overline{\mathrm{A}}}=1.91 \sqrt{\frac{\overline{\mathrm{O}}}{\mathrm{S}}}
$$

where $S$ is the speckle size, measured as the area under the normalized autocovariance function of the amplitude distribution.

\section{- signal-to-noise ratio of a difference signal}

The point signal-to-noise ratio as defined above is of limited importance in signal detection tasks, where two noisy signal levels have to be distinguished. This is because the point signal-to-noise ratio does not relate the standard deviations of the two signals to the difference in mean level. In this respect, the signal-to-noise ratio of the difference signal is more useful. Let two amplitude distributions $A_{1}(y)$ and $A_{2}(y)$ produce the respective $N$ distributions $N_{1}(y)$ and $N_{2}(y)$. The signal-to-noise ratio of the difference signal is defined as: 


$$
\mathrm{SNR}_{\Delta \mathrm{N}} \stackrel{\text { def }}{=} \frac{\left|\bar{N}_{1}-\bar{N}_{2}\right|}{\sqrt{\operatorname{var}\left(N_{1}\right)+\operatorname{var}\left(N_{2}\right)}}
$$

Substitution of Eqs. (5.8) and (5.19), and using $\bar{N} \approx \bar{O}$ yields:

$$
\begin{aligned}
\operatorname{SNR}_{\Delta N} & =\sqrt{\frac{\overline{O_{1}}}{L_{N}}} \cdot \frac{1}{\sqrt{\left|1-2^{-q / 2}\right|}} \cdot \frac{\left|\left(\overline{A_{2}} / \overline{A_{1}}\right)^{q}-1\right|}{\sqrt{\left(\overline{A_{2}} / \overline{A_{1}}\right)^{q}+1}} \\
& =\sqrt{\frac{\overline{O_{2}}}{L_{N}}} \cdot \frac{1}{\sqrt{\left|1-2^{-q / 2}\right|}} \cdot \frac{\left|\left(\overline{A_{1}} / \overline{A_{2}}\right)^{q}-1\right|}{\sqrt{\left(\overline{A_{1}} / \overline{A_{2}}\right)^{q}+1}}
\end{aligned}
$$

As expected, the $S N R_{\Delta N}$ depends on the number of independent correlation cells per mean window as well as on the difference in mean amplitude level.

\subsubsection{Transient response}

The usefulness of level-dependent spatial summation as a $2 \mathrm{D}$ imaging technique largely depends on the way edges between different structures in the image are preserved. The step function provides an indication of the degree of edge blurring. Application of the $2 \mathrm{D}$ algorithm to the part of the stationary amplitude distribution $A(x, y)$ for which $x=0$ and $y \geq 0$, gives the following expected $N$ distribution along the line $x=0$ :

$$
\begin{array}{ll}
\overline{N(0, \infty)}=2 \overline{d_{y}} \\
\overline{N(0,+y)}=\overline{d_{y}}+1 / 2+\sum_{n=1}^{y} \overline{B(0, n)} \quad(y \geq 1) \\
\overline{N(0,0)}=\overline{d_{y}}+1 / 2 \\
\overline{N(0,-1)}=\overline{d_{y}}-1 / 2 \\
\overline{N(0,-y)}=\overline{d_{y}}-1 / 2-\sum_{n=1}^{y-1} \overline{B(0, n)} \quad(y \geq 2) \\
\overline{N(0,-\infty)}=0
\end{array}
$$

where Boolean variable $B$ is defined by the $2 \mathrm{D}$ analog of Eq. (5.12): 


$$
B(0, n)=\left\{\begin{array}{lll}
0 & \text { if } & d_{y}(0, n)<n \Rightarrow p(B(y)=0)=p\left(d_{y}(0, n)<n\right) \\
1 & \text { if } & d_{y}(0, n) \geq n \Rightarrow p(B(y)=1)=p\left(d_{y}(0, n) \geq n\right)
\end{array}\right.
$$

so that:

$$
\overline{B(0, n)}= \begin{cases}e^{-\frac{\left(n / \sqrt{\frac{c}{4 k}}\right)^{4 / q}}{2 \psi}} & (q>0) \\ 1-e^{-\frac{\left.n / \sqrt{\frac{c}{4 k}}\right)^{4 / q}}{2 \psi}} & (q<0)\end{cases}
$$

An expression for $\overline{d_{y}}$ can be derived in a similar way as the expression for $\bar{O}$ (Eq. (5.7)):

$$
\overline{d_{y}}=\sqrt{\frac{c}{4 k}} \cdot(2 \psi)^{\frac{q}{4}} \cdot \Gamma\left(\frac{q}{4}+1\right) \quad(q>-4)
$$

Figure 5.1 shows the resulting $\bar{N}$ distribution, as described by Eq. (5.25), for a number of particular values of exponent q. On the basis of Eq. (5.28), parameter $c$ was

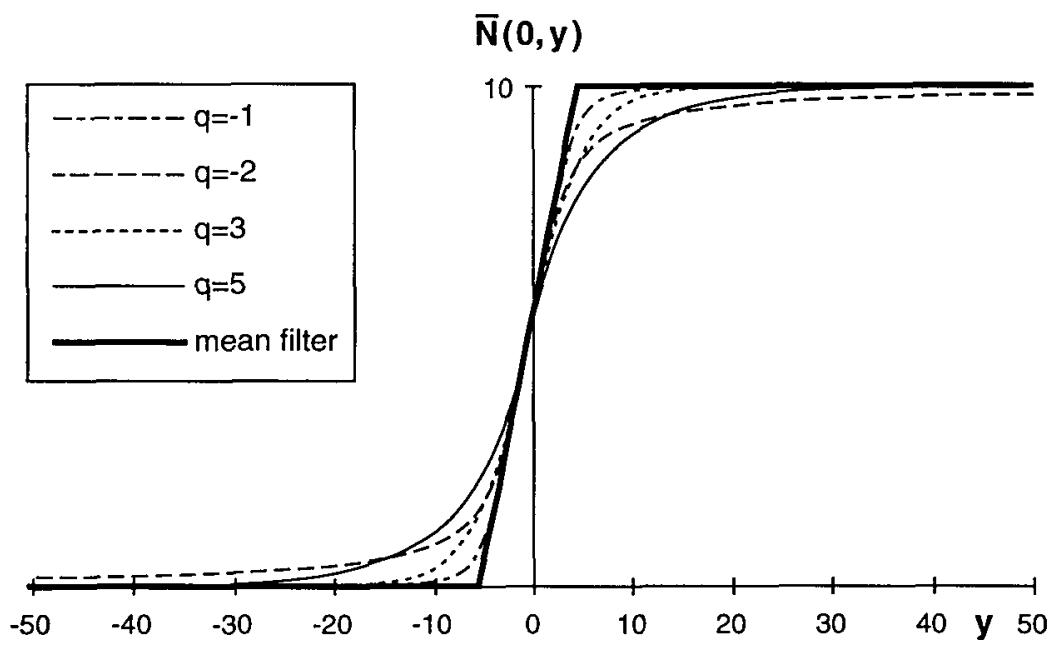

Figure 5.1. The expected transient response for four different values of exponent $q$. The algorithm is adjusted to yield $2 \overline{d_{y}}=10$. The bold line shows the expected response of a mean filter with a fixed window size of 10 points in the $y$-direction. 
adjusted for each $q$ to produce always $\overline{d_{y}}=5$. The bold line shows, as a reference, the expected response of a moving average filter with a fixed window length equal to $2 \overline{d_{y}}$. It is found that the transient response of the algorithm for level-dependent spatial summation is comparable to the response of the moving average filter. The algorithms produce the same slope at $y=0$. Only in the case of large $q$ 's $(q>5)$ does the transition in the $N$ distribution progress considerably smoother at $y \neq 0$ than the output of the moving average filter. These observations allow a meaningful comparison between the present algorithm and the $\mathrm{L}_{2}$-mean filter in lesion detection tasks.

\subsection{Experimental verification}

Three sets of five independent, one-dimensional, stationary envelope signals were generated on a PC. All signals contained $2^{19}$ amplitude points. Those in the first group were characterized by a speckle size $S$ equal to 1 sample point (white noise). The second and third set contained signals with $S$ equal to 4.5 and 9.1 sample points, respectively. Simulations were carried out by convolving a two-dimensional rf point spread function along a single scan line with random particle distributions (Bamber and Dickinson, 1980), and detecting the envelopes using the Hilbert

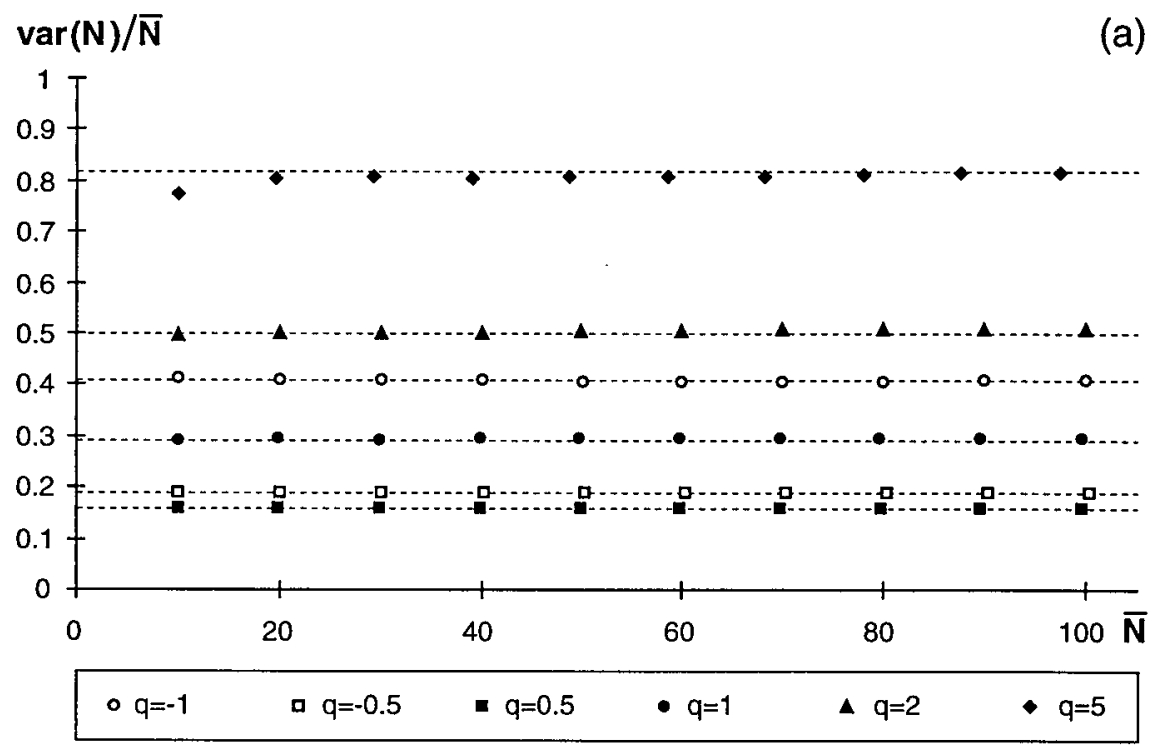

Figure 5.2. The relation measured between the ratio $\operatorname{var}(N) / \bar{N}$ and $\bar{N}$ for a number of exponents $q$. The speckle size equals 1 sample point in (a), 4.5 sample points in (b) and 9.1 sample points in (c). Each symbol represents the average results over 5 independent signals. In (a), the dashed lines indicate the theoretical behavior as summarized in table 5.1 . 

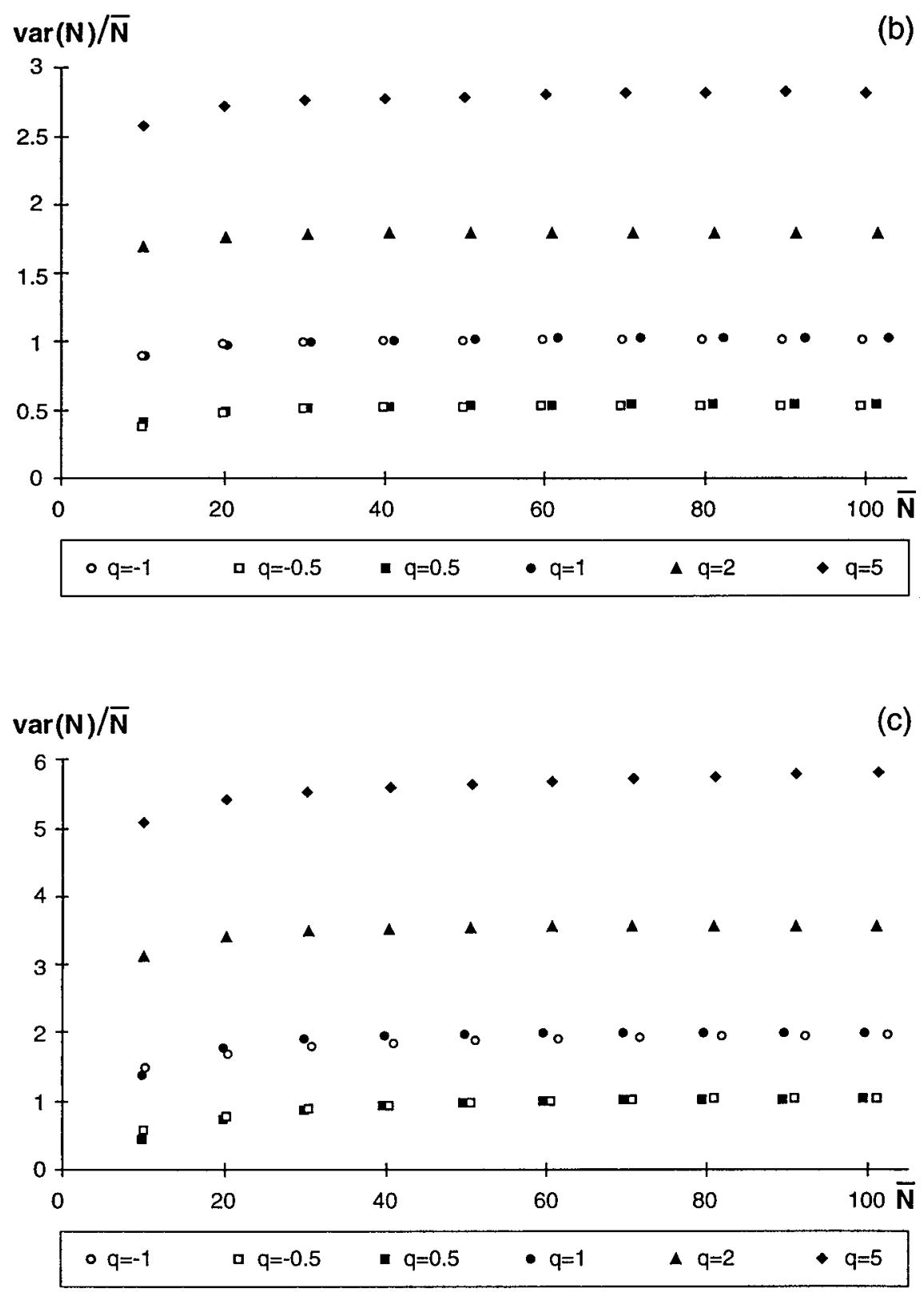

Figure 5.2. - continued 
transform. It is noted that, normally, this method is used to generate spatial speckle distributions. However, by restricting the convolution to a single scan line, also one-dimensional speckle patterns can be generated. A $\chi^{2}$ goodness-of-fit test (Daniel, 1978) proved that none of the signal distributions deviated significantly from the Rayleigh distribution, characterizing the fully developed speckle pattern (Wagner et al., 1983).

The algorithm was successively applied to the three sets of signals. Using Eq. (5.8), the expected mean $N$ value could be adjusted by varying the parameter $c$. In this way, the algorithm was set to produce, for each of the 15 input signals, 10 different mean $N$ values, following the series 10, 20, 30, .., 100 points. Afterwards, the actual $\bar{N}$ and $\operatorname{var}(N)$ were measured. To avoid border effects, the first and last 20 percent of the output signal points were excluded from the statistical calculations. Figure 5.2 shows the average results per group. The ratio $\operatorname{var}(N) / \bar{N}$ is plotted versus the measured $\bar{N}$ for a number of exponents $q \geq-1$ (for $q<-1$, the stationary state cannot be entirely achieved since $\operatorname{var}(O)$ is then infinite, as indicated by Eq. (5.10)). Every single symbol represents a result averaged over the 5 signals within one group. For none of the symbols, the standard deviation of the mean $N$ value measured exceeded 2.7 percent.

According to Eq. (5.19), the ratio $\operatorname{var}(N) / \bar{N}$ is expected to be a constant, independent of $\bar{N}$. The precise value of the ratio is found by substituting variable $L_{N}$ in Eq. (5.19). However, only for the first set of signals (white noise), $L_{N}$ is known to equal 1 sample point. For this set, the theoretical values of $\operatorname{var}(N) / \bar{N}$ are indicated in figure 5.2 -a by the horizontal, dashed lines. Obviously, the values measured

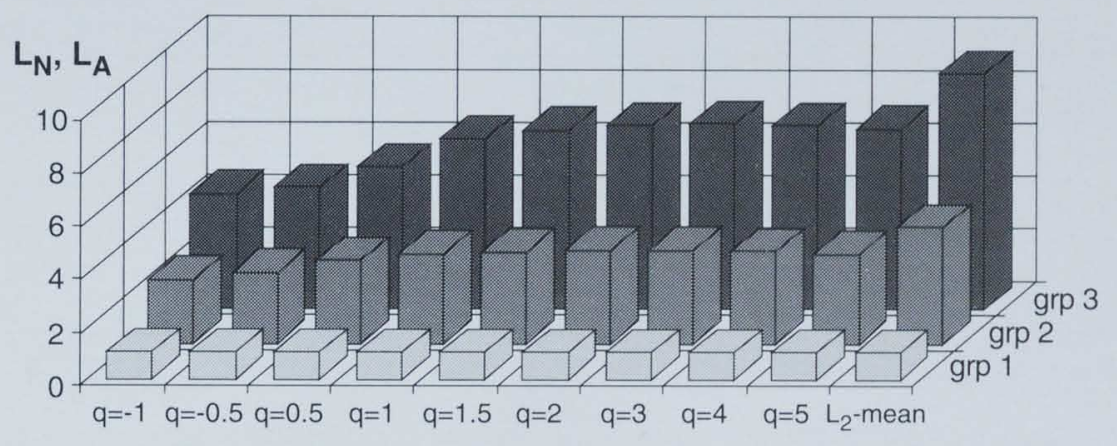

Figure 5.3. Estimates of the correlation cell sizes $L_{N}$ (in sample points) based on the data presented in figure 5.2. The right-most column shows, as a reference, the estimates of $L_{A}$ as seen by the $\mathrm{L}_{2}$-mean filter. The speckle size equals 1 sample point for grp 1, 4.5 sample points for grp 2 and 9.1 sample points for grp 3 . 
closely approach the theoretically expected behavior. For the other two groups, an assessment was made of $L_{N}$ at $\bar{N} \approx 30$, based on the data presented in figure 5.2. According to Eq. (5.19), the division of a value of $\operatorname{var}(N) / \bar{N}$ in group 2 or 3 by the corresponding value in group 1 , yields an estimation of $L_{N}$. The results are shown in figure 5.3, with exponent $q$ ranging between -1 and 5 . As a reference, the correlation lengths $L_{A}$ as experienced by the $L_{2}$-mean filter (closely approximating $S$ ) are shown in the right-most column. These values were obtained by applying a 30-point $\mathrm{L}_{2}$-mean filter, instead of the present algorithm, to the three sets of signals. Apparently, $L_{N}$ depends on the exponent $q$, but for all $q$ 's, $L_{N}$ remains smaller than $L_{A}$.

A second experiment was carried out to measure $S N R_{\Delta N}$. For this purpose, each of the 15 envelope signals available was split into two separate, equally sized parts, one serving as "background" signal, the other as "foreground" signal. Every foreground signal was rescaled with respect to its related background signal, thus achieving a difference in mean amplitude level of $3 \mathrm{~dB}: \bar{A}_{\text {foregr }}=0.7 \bar{A}_{\text {backgr }}$. Application of the algorithm to a foreground signal and, thereafter, without changing the setting, to its related background signal, yields two output $N$ distributions. According to Eq. (5.8), the two output signals will differ in mean $N$ level. If exponent

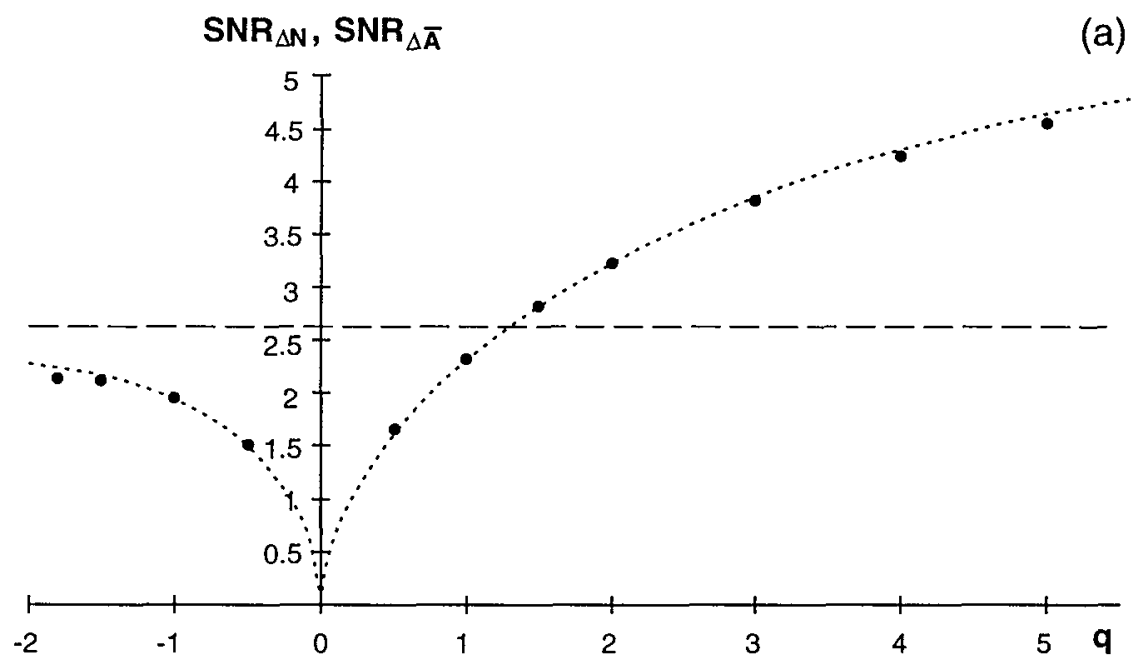

Figure 5.4. $S N R_{\triangle N}$ as a function of exponent $q$. The mean window size adjusted is 30 sample points. The speckle size equals 1 sample point in (a), 4.5 sample points in (b) and 9.1 sample points in (c). Each symbol represents the average results over 5 independent difference signals. As a reference, the horizontal dashed lines show $S N R_{\triangle \bar{A}}$ as produced by a 30-point $L_{2}$-mean filter. In (a), the dashed curve indicates the theoretical behavior as predicted by Eq. (5.24). 
$q>0, \bar{N}_{\text {backgr }}$ will be larger than $\bar{N}_{\text {foregr }}$, while for $q<0$, the opposite is true. To enable a comparison of the behavior of the algorithm at positive $q$ with that at negative $q$, parameter $c$ was adjusted every turn (using Eq. (5.8)), such that the largest mean $N$ value of each pair of output signals equaled 30 . That is, $\bar{N}_{\text {backgr }}=30$ if $q>0$ and
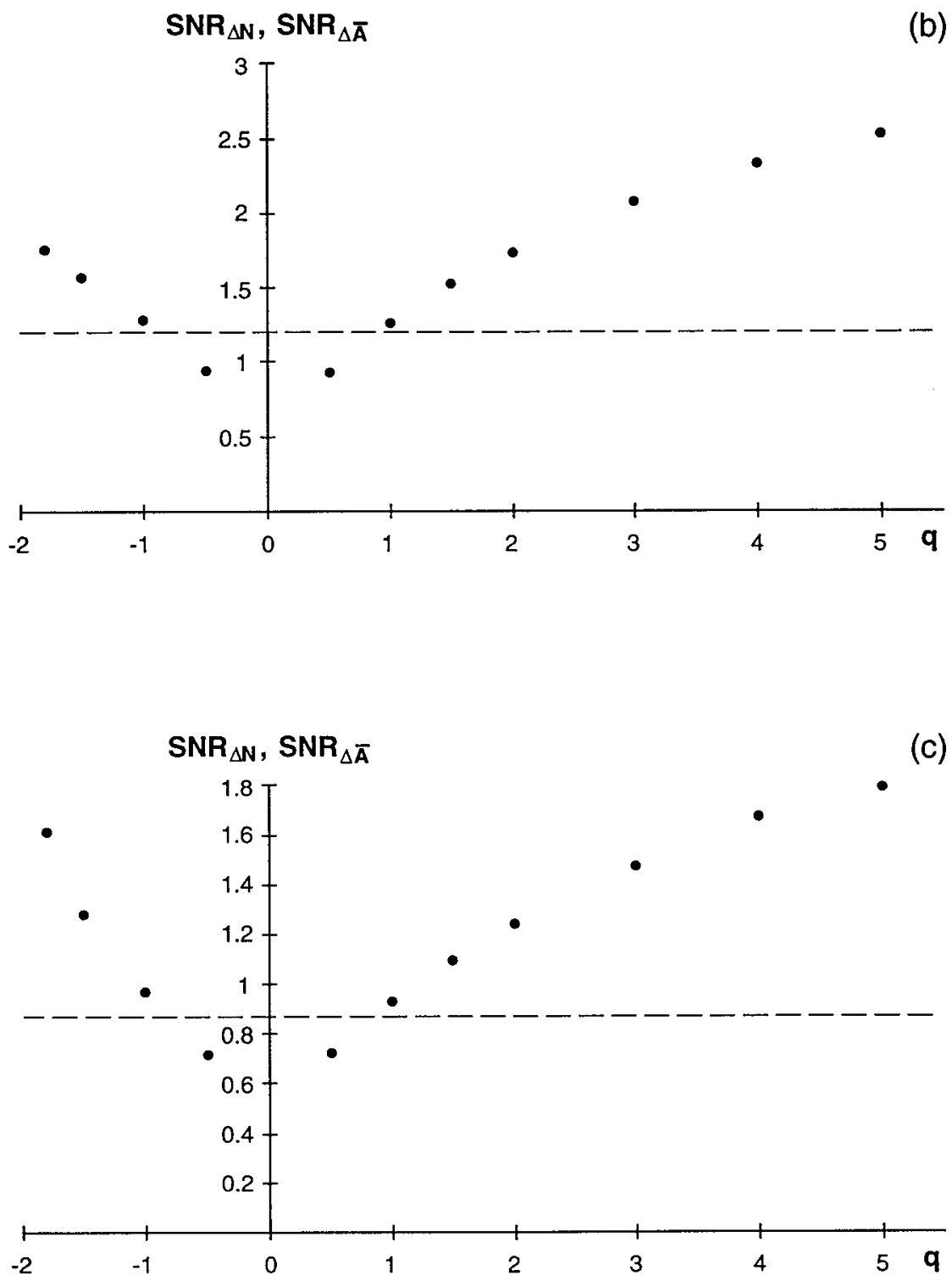

Figure 5.4. - continued 
$\bar{N}_{\text {foregr }}=30$ if $q<0$. This way, the $S N R_{\Delta N}$, as defined in Eq. (5.23), was calculated for each pair of signals, and for various $q$ 's ranging between -1 and 5 . The average results per group are shown in figure 5.4. Again, knowledge of $L_{N}$ in the first group enabled the theoretical characteristic to be calculated with Eq. (5.24). Obviously, the values measured closely agree with the theoretical curve (dashed line in figure 5.4a). As a reference, the horizontal dashed lines show for each group the mean $S N R_{\Delta \bar{A}}$ as produced by a 30-point $\mathrm{L}_{2}$-mean filter. Note that in figure 5.4-a, $S N R_{\Delta N}<$ $S N R_{\Delta \bar{A}}$ for $q \leq-1$ and $q=1$, while for the same exponents in figures 5.4-b and 5.4-c, the opposite is true. Moreover, the relative difference between $S N R_{\Delta N}$ at $q \leq-1$ as compared with $S N R_{\Delta N}$ at $q=5$ is larger in group 1 (white noise) than in the other two groups. These observations are consistent with the estimations of $L_{N}$ presented in figure 5.3 and the expectation that $S N R_{\Delta N}$ is inversely proportional to the square $\operatorname{root}$ of $L_{N}($ Eq. (5.24)).

\subsection{Lesion detection}

Two regions are identified in a lesion image as modeled by signal detection theory: one consisting of a signal plus noise (the lesion), the other only containing noise (the background). A complete description of how well the signal plus noise part can be distinguished from the noise only part is provided by the ROC curve, being a plot of the false positive fraction (FPF, the fraction of background points falsely recognized as lesion points) versus the true positive fraction (TPF, the fraction of correctly identified lesion points) as a function of a threshold applied to the image. The area $A_{R O C}$ under the ROC curve has been recommended as a one-number summary of the curve (Green and Swets, 1988; Swets and Pickett, 1982). It equals the fraction of correct answers in a two-alternative forced choice experiment and ranges from 0.5 in the pure guessing case to 1 in the perfect performance case. In the present study, $A_{R O C}$ is used to compare the performance of the $\mathrm{L}_{2}$-mean filter with the algorithm for level-dependent spatial summation in a lesion detection task.

Ten independent realizations of the same lesion were generated on a PC by convolving a two-dimensional rf point spread function with spatial particle distributions. The resulting images all contain a central, circular, hypoechoic (negative contrast) lesion with a diameter of $2 \mathrm{~cm}$ in a homogeneous background of $6 \times 6 \mathrm{~cm}^{2}$. Lesion scatter strength is $3 \mathrm{~dB}$ below background scatter strength. Particle density equals 26 per resolution cell. The number of parallel envelope lines is 113 , the number of sample points per envelope line is 1024 . The speckle size equals 3.6 sample points $(\approx 2 \mathrm{~mm})$ in the lateral direction and 7.7 points $(\approx 0.5 \mathrm{~mm})$ in the axial direction. In the same way, a similar set of ten independent hyperechoic (positive con- 
trast) lesions was generated. In this set, lesion scatter strength is $3 \mathrm{~dB}$ above background scatter strength.

The present algorithm was compared in two different ways with the $\mathrm{L}_{2}$-mean filter. First, the algorithm for level-dependent spatial summation was adjusted to give approximately equal edge blurring as the $L_{2}$-mean filter. That is, using Eq. (5.28), the mean lateral and axial window dimensions $2 \overline{d_{x}}$ and $2 \bar{d}_{y}$ (as averaged over either the background or the lesion area, whichever produces the largest values) were set to equal the respective fixed dimensions of the $\mathrm{L}_{2}$-mean filter. Windows were clipped at $O_{\max }=5000$ points. This prevented incidental outliers to become extremely large, which may especially occur for $-2<q<-1$ (where $\operatorname{var}(O)$ is theoretically infinite). It is noted that, due to the clipping of the windows, the mean window dimensions measured afterwards could be less than the values aimed at. The procedure followed was that if the difference turned out to be larger than 5 percent, parameter $c$ was readjusted and the convolution was carried out a second time. The two algorithms were successively applied to the two sets of ten spatial amplitude distributions. This yielded, for each set, ten independent $N$ distributions and ten independent $\mathrm{L}_{2}$-mean filtered amplitude distributions. For each of these processed signal distributions obtained (processed "lesion images"), an ROC curve was estimated: 100 different thresholds were applied to the distribution, ranging between the minimum and maximum signal levels present. Each threshold yielded a pair (FPF, TPF). All pairs together made up one ROC curve. Numerical integration of the curve yielded an estimate of $A_{R O C}$. The results are presented in figure 5.5. For a number of different exponents $q$, the upper part shows the median value of $A_{R O C}$ over the ten hypoechoic lesion images, as well as the first and third quartiles. The lower part shows the results over the ten hyperechoic lesion images. For figure 5.5a, the ratio $k=d_{x} / d_{y}$ approximated 0.5 , so that the number of speckle cells covered by a window in the axial direction equals the number in the lateral direction. The $\mathrm{L}_{2}$-mean filter had fixed window dimensions of 7 points in the lateral direction and 15 points in the axial direction. Its performance is described in figure 5.5 -a by the horizontal lines. For both the negative and positive contrast lesions, a difference in the median value of $A_{R O C}$ as produced by the two methods of the order of 0.1 can be observed for $q<-1.5$ and $q \geq 3$. Moreover, the variation in the performance, expressed as the difference between the first and third quartiles of $A_{R O C}$, is smallest for the present method. For figure 5.5-b, $k$ approximated 0.1 , so that all windows are square if the dimensions are expressed in millimeters. The $\mathrm{L}_{2}$-mean filter had fixed window dimensions of 3 points in the lateral direction and 33 points in the axial direction. The performance is found to be slightly better for both methods (except for $|q|=0.5$ ) as compared with figure 5.5-a. Further, it is observed in both figures 5.5-a and $5.5-\mathrm{b}$, that the present algorithm, adjusted with a negative $q$, performs slightly 

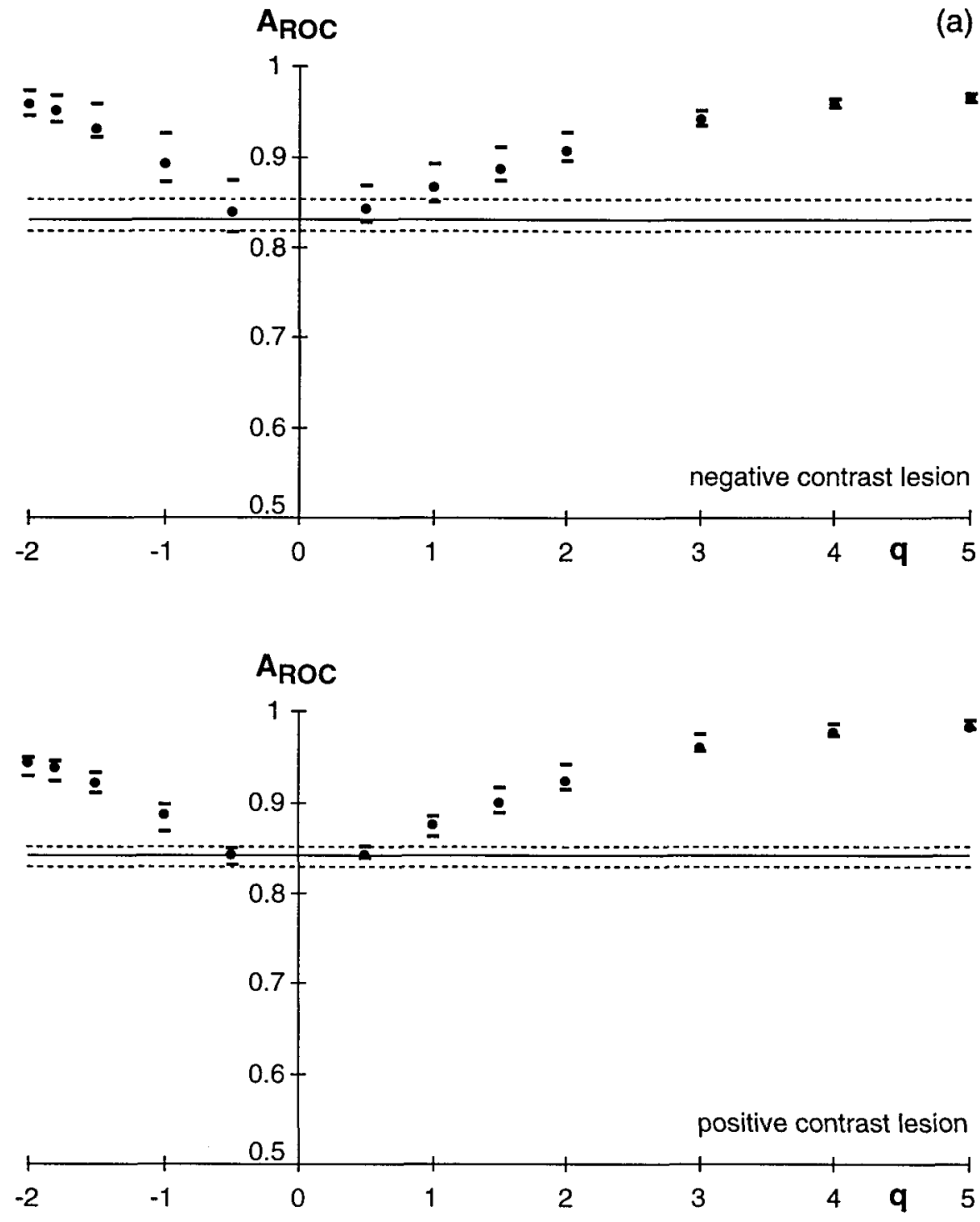

Figure 5.5. $A_{R O C}$ as a function of exponent $q$ for negative contrast lesions (upper part) and positive contrast lesions (lower part). Each dot represents the median value over 10 images. The two bars enclosing each dot indicate the first and third quartiles of $A_{R O C}$. The horizontal lines show the performance of the $\mathrm{L}_{2}$-mean filter (continuous: median, dashed: quartiles). The algorithm was adjusted to produce equal mean window dimensions $2 \overline{d_{x}}$ and $2 \overline{d_{y}}$ as the fixed dimensions of the $\mathrm{L}_{2}$-mean filter: $7 \times 15$ points in (a) and $3 \times 33$ points in (b).

better for negative contrast lesions than for positive contrast lesions, while the 

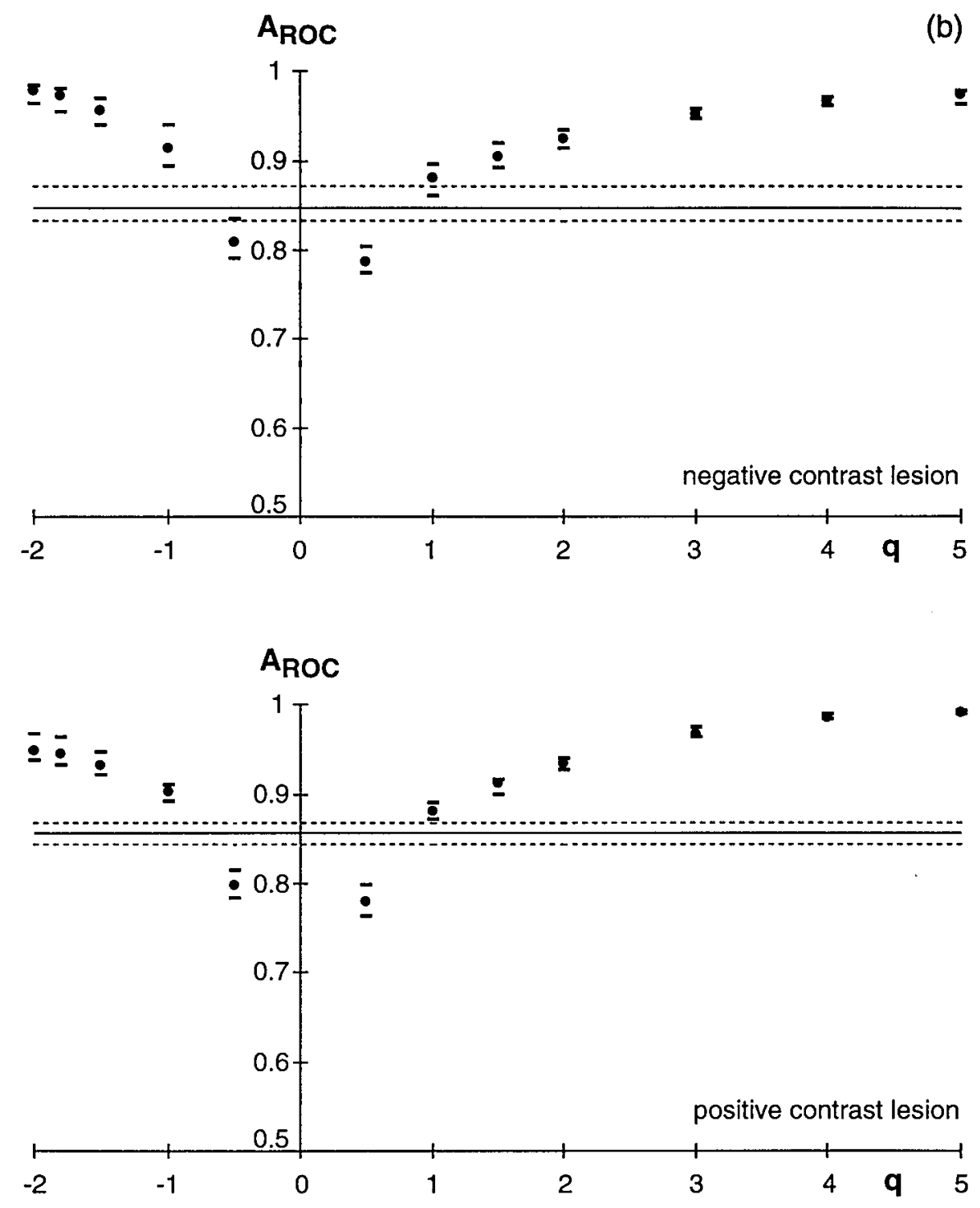

Figure 5.5. -continued

algorithm, adjusted with a positive $q$, shows the opposite behavior. This effect is most obvious for power $q \leq-1.5$ and $q \geq 2$.

In the second comparison method, the same ROC analysis was carried out as described above. However, this time, the algorithm for level-dependent spatial 

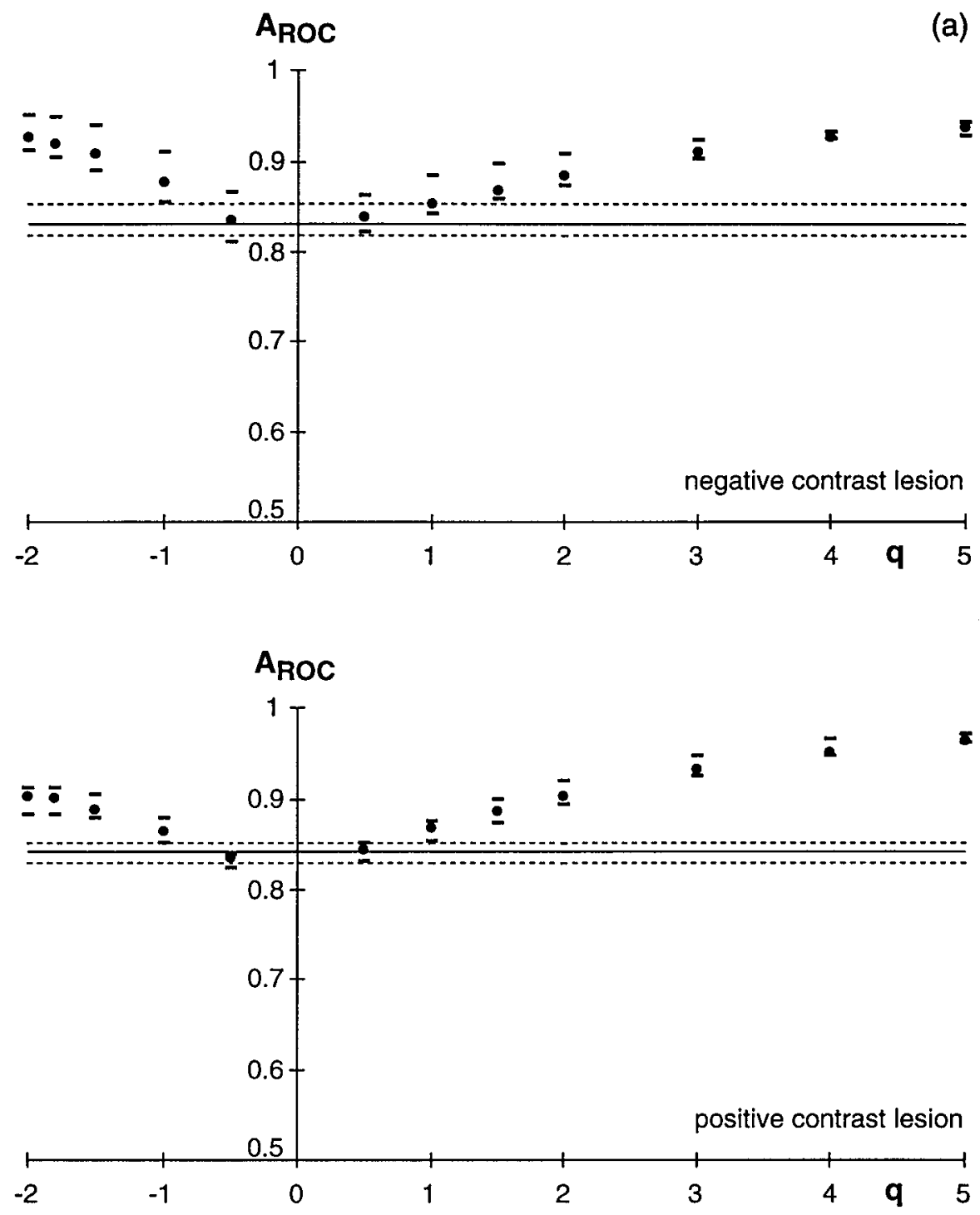

Figure 5.6. Same as figure 5.5, except that the algorithm was adjusted to produce a mean $N$ value equal to 100 points. In (a), $k=0.5$ and the $\mathrm{L}_{2}$-mean filter had a $7 \times 15$ points window. In (b), $k=0.1$ and the $\mathrm{L}_{2}$-mean filter had a $3 \times 33$ points window.

summation was adjusted, using Eq. (5.8), to produce a mean $N$ value (again, as averaged over either the background or the lesion area, whichever gives the largest $\bar{N}$ ) equal to the fixed window area of the $L_{2}$-mean filter. As a result, the number of 

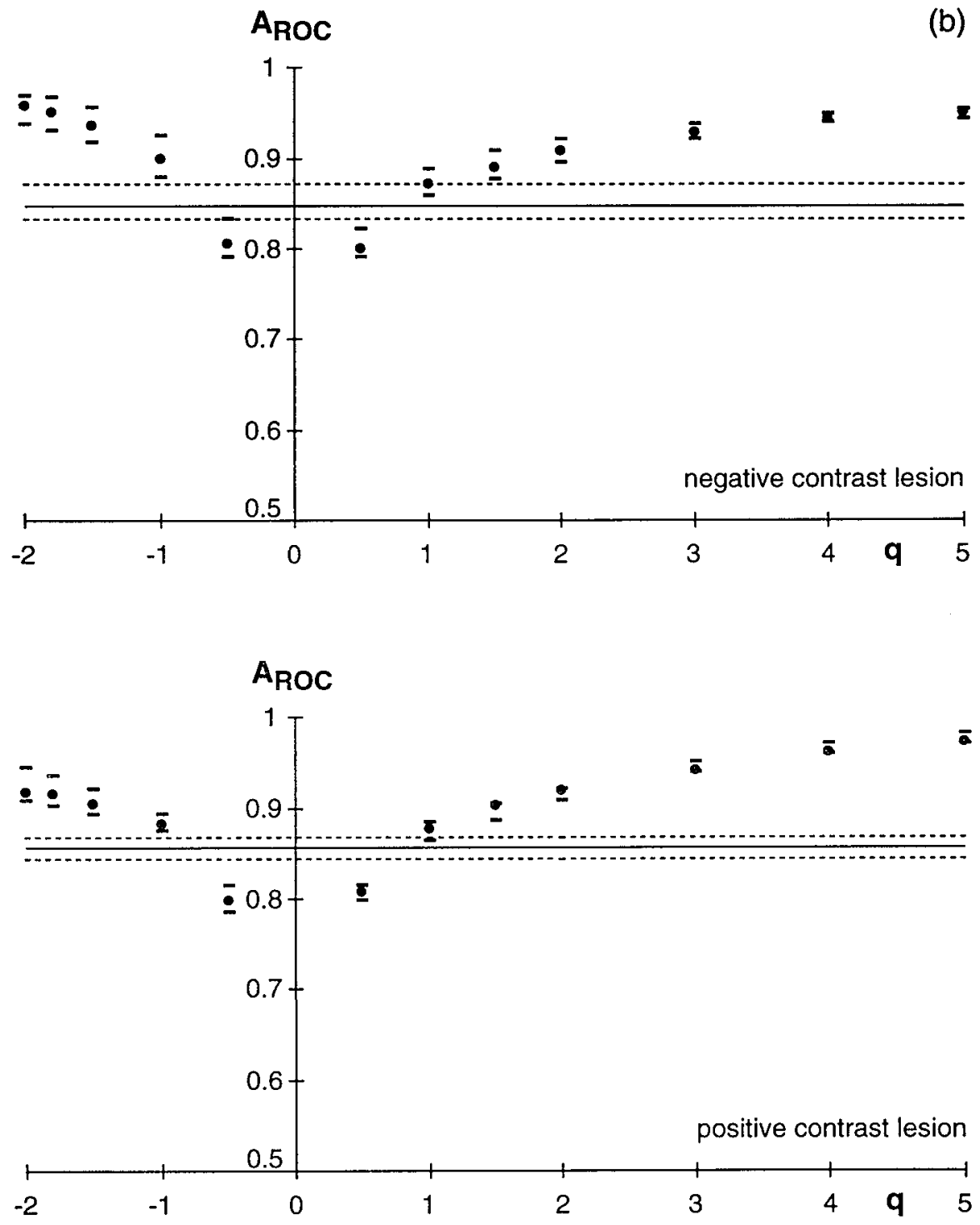

Figure 5.6. - continued

summations involved in the convolution of Eq. (5.1) is comparable to the number of summations required by the $\mathrm{L}_{2}$-mean filter. The results are presented in figures 5.6-a and 5.6-b (upper part: negative contrast lesions, lower part: positive contrast lesions). In both figures, $\bar{N}$ equaled 100 , but in figure 5.6-a, $k=0.5$ while in figure $5.6-\mathrm{b}, k=0.1$. The $\mathrm{L}_{2}$-mean filter of figure $5.6-\mathrm{a}$ is the same as the one of figure $5.5-\mathrm{a}$ 
while the $\mathrm{L}_{2}$-mean filter of figure 5.6-b corresponds to the one of figure 5.5-b. Both $\mathrm{L}_{2}$-mean filters had a fixed window area approximately equal to 100 . Again, a difference in the median value of $A_{R O C}$ as produced by the two methods of the order of 0.1 (but slightly smaller than in the previous experiment) can be observed for $q \geq 3$. In addition, a similar effect of factor $k$ on the performance is found: square windows (dimensions in millimeters) do better than windows adapted to the speckle size (in sample points). Further, it is again observed that, for negative $q$ 's, the performance of the present algorithm is best for negative contrast lesions, while for positive $q$ 's, the performance is best for positive contrast lesions. A final remark is made concerning edge blurring. It is noted that the product $4 \overline{d_{x}} \cdot \overline{d_{y}}$ can be considerably smaller than $\bar{O}=4 \overline{d_{x} \cdot d_{y}}$, depending on the value of exponent $q$. Consequently, in

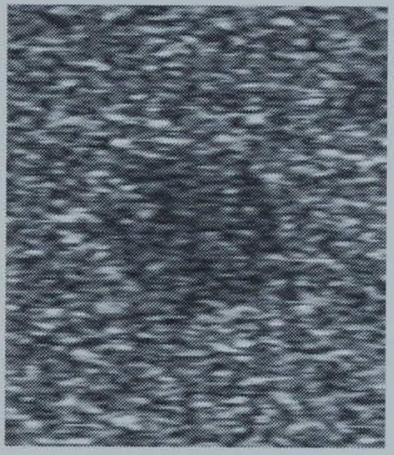

(a)

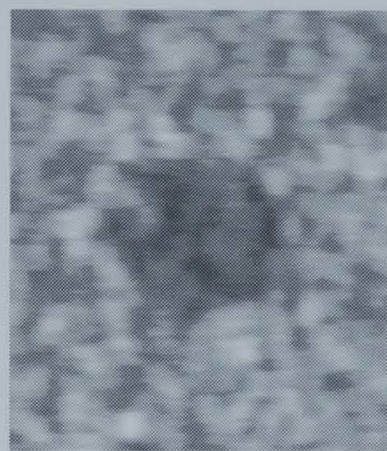

(b)

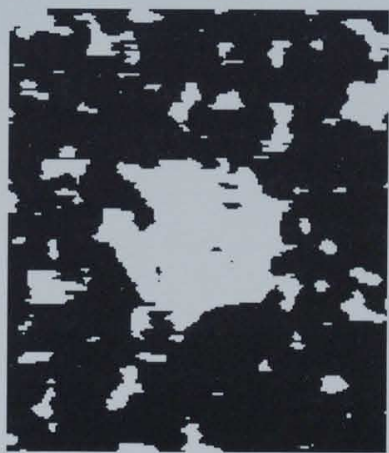

(d)

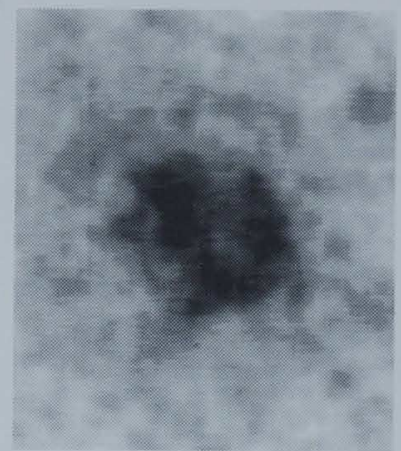

(c)

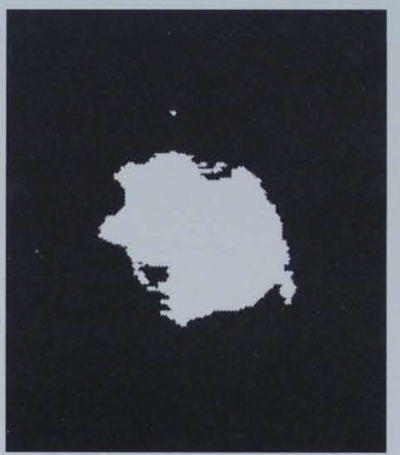

(e)

Figure 5.7. (a) Simulated lesion image. (b) Response of a $5 \times 55$ points $\mathrm{L}_{2}$-mean filter. (c) Response of the algorithm for level-dependent spatial summation on inverted gray scale, with $q=$ $-2, \bar{N}_{\text {lesion }}=277$ and $k=0.1$. (d) Binary image obtained by thresholding image (b). (e) Binary image obtained by thresholding image (c). In both the images (d) and (e), 87 percent of all lesion points were detected correctly. 
the second experiment, edge blurring tended to be smaller in the $N$ distribution than in the output of the $\mathrm{L}_{2}$-mean filter.

The difference in performance is concretized in figures 5.7 and 5.8 for one of the simulated lesion images and for one particular setting of the algorithms. Figure 5.7-a shows the central part of the original image $\left(4.7 \times 5.4 \mathrm{~cm}^{2}\right)$. Application of the $\mathrm{L}_{2}$-mean filter to the corresponding amplitude distribution yielded the image of figure 5.7-b. The window dimensions of the filter equaled 5 sample points in the lateral direction and 55 points in the axial direction. Application of the algorithm for leveldependent spatial summation, with exponent $q=-2$, to the original amplitude distribution, resulted in the image of figure 5.7-c (shown on inverted gray scale). The ratio $k$ of the lateral and axial window dimensions equaled 0.1 and $\bar{N}_{\text {lesion }}$ equaled 277 , approximating the window area of 275 of the $\mathrm{L}_{2}$-mean filter. The mean axial window size $2 \overline{d_{y}}$ was 33 and the mean lateral window size $2 \overline{d_{x}}$ was 3.3 . Observe that the lesion boundary appears to be sharper in figure 5.7-c than in figure 5.7-b. Figure 5.8 shows the ROC curves corresponding to the distributions shown in figures 5.7-a to 5.7-c. $A_{R O C}$ equals 0.673 for the original amplitude distribution, 0.937 for

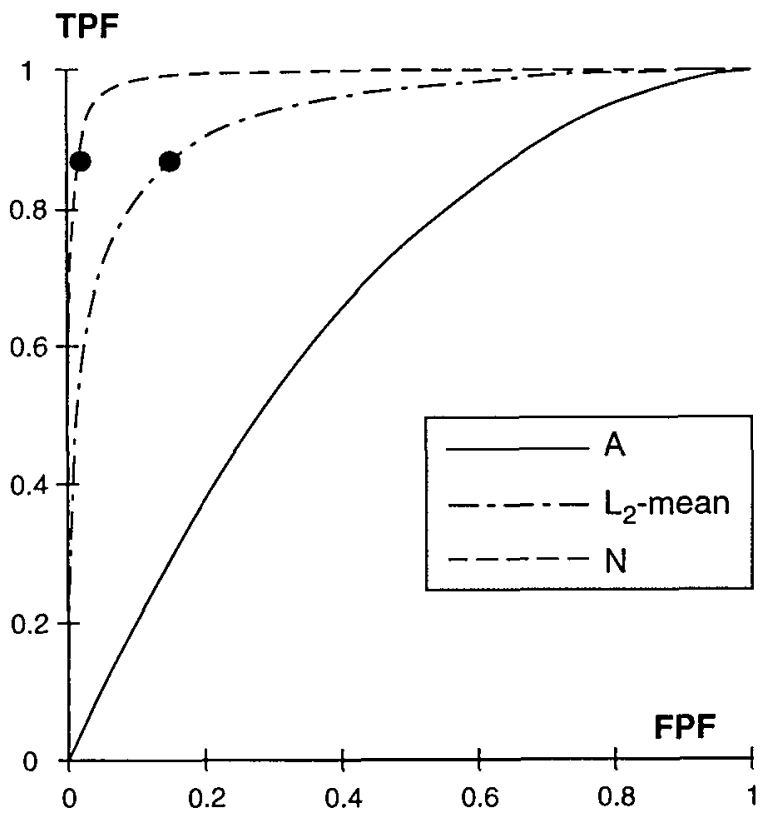

Figure 5.8. Three ROC curves corresponding to the images of figures 5.7-a, -b and -c. The two dots indicate the locations on the ROC curves that correspond to the binary images of figures 5.7$\mathrm{d}$ and -e. 
the output of the $\mathrm{L}_{2}$-mean filter and 0.993 for the $N$ distribution. The difference in $A_{R O C}$ between the two processed distributions seems to be small. However, if $A_{R O C}$ is converted into the percentage mistakes in a two-alternative forced choice experiment, it follows that the algorithm for level-dependent spatial summation makes 0.7 percent errors while the $\mathrm{L}_{2}$-mean estimator fails in 6.3 percent of all cases, which is a factor 9 higher. Figures 5.7-d and 5.7-e present the difference in performance in another way. The binary images were obtained by applying a threshold to the processed images of figures 5.7-b and 5.7-c, respectively. All pixels exceeding the threshold were detected as background pixels. They are drawn black in the binary images. The remaining pixels were recognized as lesion pixels and are white in the binary images. For both the $\mathrm{L}_{2}$-mean image and the $N$ image, the

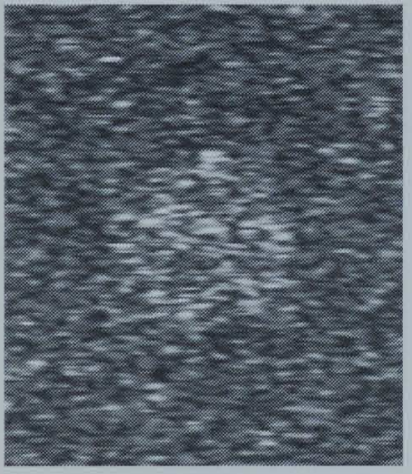

(a)

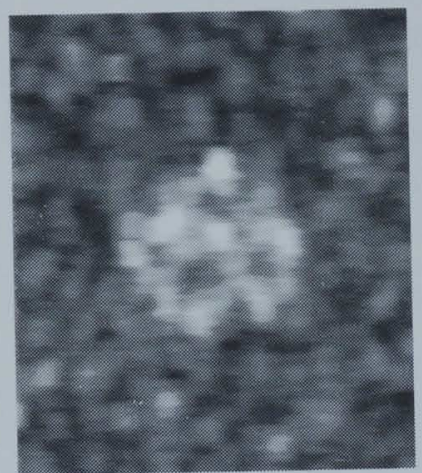

(b)

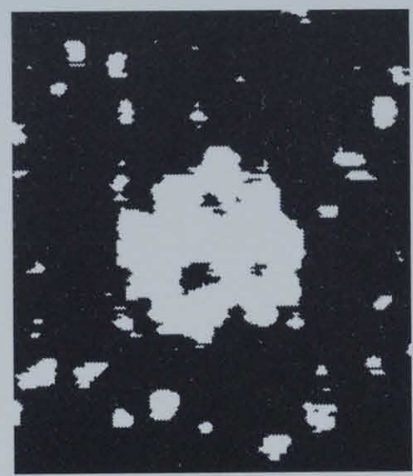

(d)

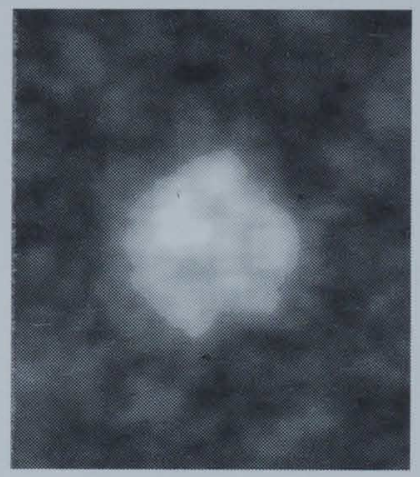

(c)

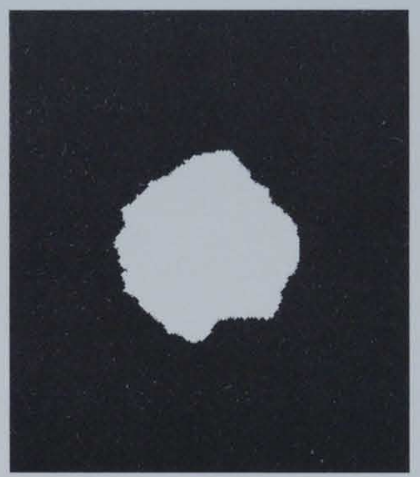

(e)

Figure 5.9. (a) Central part of one of the simulated positive contrast lesion images. (b) Response of a $5 \times 55$ points $\mathrm{L}_{2}$-mean filter. (c) Response of the algorithm for level-dependent spatial summation, with $q=5, k=0.1$ and with $\bar{N}_{\text {lesion }} \approx 275$. (d) Binary image obtained by thresholding image (b). (e) Binary image obtained by thresholding image (c). 
threshold was adjusted such, that 87 percent of all lesion pixels were detected, i.e., $T P F=0.87$ for both images (see the two dots in figure 5.8). Obviously, the FPF is much smaller for the $N$ distribution than for the $\mathrm{L}_{2}$-mean distribution. As can be seen in figure 5.8, $F P F_{N}=1.4$ percent while $F P F_{L_{2} \text {-mean }}=14$ percent.

Figure 5.9 gives another example of lesion detection. Figure 5.9-a shows one of the positive contrast lesion images simulated. Figure 5.9-b displays the response of a $5 \times 55$ points $\mathrm{L}_{2}$-mean filter while figure 5.9-c shows the output of the present algorithm, adjusted with $q=5, k=0.1$ and a mean lesion window area $\bar{O}_{\text {lesion }}\left(\approx \bar{N}_{\text {lesion }}\right)$ equal to the fixed window area of the $L_{2}$-mean filter: 275 . The binary images of figures 5.9- $\mathrm{d}$ and -e were obtained by thresholding the images of figures $-\mathrm{b}$ and $-\mathrm{c}$, respectively. The threshold applied to the output of the $\mathrm{L}_{2}$-mean filter produced a TPF of 87 percent and a FPF of 7.5 percent. The threshold on the $N$ distribution yielded $T P F=94$ percent and $F P F=0.7$ percent, demonstrating the superior performance of the present algorithm.

\subsection{Discussion}

The processing performed by the present algorithm is essentially inverse to the action of a standard moving average filter like, e.g., the $L_{2}$-mean filter: in the processing scheme of the moving average filter, each point $A(x, y)$ in the input distribution contributes to a fixed number of locations (equal to the window area) in the output distribution. Thereby, the extent of the contribution is a function of $A(x, y)$. In the present processing scheme, on the contrary, each point $A(x, y)$ contributes to a variable number of locations in the output distribution, depending on the value of $A(x, y)$, while the extent of the contribution is always 1 . The latter approach has been shown to have the best performance in lesion detection tasks.

Concerning the ROC analysis, it is pointed out that the area $A_{R O C}$ under the Receiver Operating Characteristic is an objective measure of how well a single sample from the lesion can be distinguished from a single point in the background. The detectability of the lesion as a whole depends, apart from $A_{R O C}$, also, for a certain concept of the observer, on the size of the lesion (i.e., the number of independent information grains in the lesion) (Smith et al., 1983; Thijssen et al., 1988; Verhoeven and Thijssen, 1993; Wagner and Brown, 1985).

What remains to be answered is how the difference in performance between the $\mathrm{L}_{2}$-mean filter and the algorithm for level-dependent spatial summation can be explained. The capacity of the two processing methods to distinguish a lesion point 
from a background point is principally determined by two factors: the level of the signal-to-noise ratios of the difference signals they produce (Eq. (5.23)), and the behavior at edges (the more an edge is blurred, the more false recognitions in the vicinity of the edge will occur). It is observed that the performance of the present algorithm in lesion detection as a function of exponent $q$ (Figs. 5.5 and 5.6) globally agrees with the relationship between $S N R_{\Delta N}$ and $q$ (Fig. 5.4). As shown in figures 5.4-b and 5.4-c, $S N R_{\Delta N}$ is larger than $S N R_{\Delta A}$ for the majority of exponents. Only for $q$ approaching zero, the opposite is true. For a number of particular values of the exponent ( $q<-1$ and $q=1$ ), an obvious contribution is noticed of the correlation cell size $L_{N}$ to the fact that $S N R_{\Delta N}>S N R_{\Delta \bar{A}}$ for speckle sizes larger than 1 sample point. As observed (Fig. 5.3), the correlation cell size experienced by the present algorithm is smaller than the correlation cell size $L_{A}$ as seen by the $L_{2}$-mean filter. This causes the $S N R_{\Delta N}$ to degrade less than the $S N R_{\Delta \bar{A}}$ if the speckle size becomes larger than 1 sample point. Hence, although, in principle, Nyquist sampling of the amplitude distribution is sufficient, a slight increase in the ratio $S N R_{\Delta N} / S N R_{\Delta \bar{A}}$ can be gained by increasing the sample frequency up to two times the Nyquist frequency. Concerning the edge behavior (Fig. 5.1), it was found for both the positive and the negative $q$ 's that the S-shaped transitions produced by the algorithm for level-dependent spatial summation stretch over a longer distance as the modulus of $q$ becomes larger (although the slope in the center does not change). This explains why for exponents $q$ larger than 5 , the performance in lesion detection degrades although $S N R_{\Delta N}$ theoretically reaches a maximum at $q$ approaching infinity. The edge response may also account for the observation that negative $q$ 's perform less for positive contrast lesions than for negative contrast lesions, while positive $q$ 's exhibit the opposite behavior. For both positive and negative $q$ 's, the deteriorated performance occurs when the background produces, on average, larger windows than the lesion. Especially for those $q$ 's for which the S-shaped edge response has long tails ( $q$ close to -2 and $q$ close to 5 ) even relative distant background points may contribute to the interior of the lesion, this way increasing $\bar{N}_{\text {lesion }}$ and thus decreasing the lesion contrast. This effect, which will especially affect the detectability of small lesions (of a few speckle cells) can be reduced by posing an upper limit to the window size $O$. As a result, the long tails in the edge response disappear while the response in the vicinity of the edge remains unchanged. It is noted that in the opposite situation, where the lesion produces the highest $N$ level (which happens if a negative $q$ is applied to a hypoechoic lesion or a positive $q$ is applied to a hyperechoic lesion), the contrast reducing effect of the long tails in the edge response is much less severe. This is explained by the fact that the lesion, consisting of a limited number of amplitude points, produces only a very small number of windows that reach far beyond the boundary of the lesion. Consequently, enhancement of the mean background $N$ level is minimal. A final note on the edge behavior is, that the S-shaped response, to 
a certain extent, is not perceived more blurred than the straight slope of the moving average filter. This can be explained by the fact that the human visual system is more sensitive to rapid transitions (in the center of the $S$ ) than gradual ones (away from the center) (Levine, 1985), allowing a precise localization of the edge.

The factor $k$ determines the ratio of the lateral and axial window dimensions. Square windows (dimensions expressed in millimeters) were found to do better than windows having the ratio of the dimensions adapted to the speckle size (in sample points). This observation is consistent with the findings of other researchers (Kotropoulos and Pitas, 1992).

Concerning real-time implementation, it is suggested to divide the input amplitude range into a number of intervals and store for each interval the corresponding lateral and axial radii of support in a look-up table. All processing is reduced then to table indexing and the adding of 1 to the locations specified by the tabled data. The look-up table has another advantage. It automatically poses a limit to the maximum window area, so that, especially for $q<-1$, edge behavior is improved, robustness is increased and time is gained by not allowing the windows to become extremely large. Moreover, for negative $q$, the largest windows are produced by the smallest amplitudes. Since these amplitudes have a strong noise component, information loss due to the clipping of the window area is minimal.

\subsection{Conclusion}

A novel method has been described for postprocessing spatial amplitude distributions. The action is basically inverse to the processing of a standard moving average filter. The output has been shown to include information on the local mean gray level in the original image, which can be displayed as a gray level image. The theoretically predicted behavior has been experimentally confirmed. On the basis of ten hypoechoic and ten hyperechoic lesion images, it has been demonstrated that the present algorithm behaves superior to the $\mathrm{L}_{2}$-mean filter in lesion detection. An improvement in the median value of the area $A_{R O C}$ under the Receiver Operating Characteristic of the order of 0.1 was observed. The difference in performance is largest for exponent $q$ between -2 and -1 and for $q$ between 3 and 5 . The algorithm is adjusted in the same way as a moving average filter: one parameter (c) determines the mean window area, and one parameter $(k)$ determines the ratio of the lateral and axial window dimensions. If the algorithm is adjusted to produce a mean window area equal to the fixed window area of a moving average filter, the two methods use approximately the same number of summations in the processing of a frame, 
indicating that real-time implementation of the present algorithm is possible.

\section{REFERENCES}

Bamber J.C. and Daft C. (1986): Adaptive filtering for reduction of speckle in ultrasonic pulse-echo images, Ultrasonics 24, 41-44.

Bamber J.C. and Dickinson R.J. (1980): Ultrasonic B-scanning: a computer simulation, Phys. Med. Biol. 25, 463-479.

Bartrum Jr. R.J. and Crow H.C. (1980): Ultrasound echo averaging: A simple method for improving image perception, J. Clin. Ultrasound 8, 63-64.

Collaris R.J. and Hoeks A.P.G. (1994): Ultrasonic B-mode image enhancement based on level-dependent spread functions, Ultrasonic Imaging 16, 205-230.

Daniel W.W. (1978): Applied Nonparametric Statistics (Houghton Mifflin Company, Boston).

Dickinson R.J. (1982): Reduction of speckle in ultrasound B-scans by digital processing, in Acoustical Imaging 12, Ash E.A. and Hill C.R., Eds., Plenum Press, New York, pp. 213-224.

Green D.M. and Swets J.A. (1988): Signal Detection Theory and Psychophysics (Peninsula Publishing, Los Altos).

Koo J.I. and Park S.B. (1991): Speckle reduction with edge preservation in medical ultrasonic images using a homogeneous region growing mean filter (HRGMF), Ultrasonic Imaging 13, 211-237.

Kotropoulos C. and Pitas I. (1992): Optimum nonlinear signal detection and estimation in the presence of ultrasonic speckle, Ultrasonic Imaging 14, 249-275.

Levine M.D. (1985): Vision in Man and Machine (McGraw-Hill, New York).

Loupas T., McDicken W.N. and Allan P.L. (1989): An adaptive weighted median filter for speckle suppression in medical ultrasonic images, IEEE Trans. Circuits Systems 36, 129-135.

Magnin P.A., Von Ramm O.T. and Thurstone F.L. (1982): Frequency compounding for speckle contrast reduction in phased array images, Ultrasonic Imaging 4, 267-281.

Melton Jr. H.E. and Magnin P.A. (1984): A-mode speckle reduction with compound frequencies and compound bandwidths, Ultrasonic Imaging 6, 159-173.

Pitas I. and Venetsanopoulos A.N. (1986): Edge detectors based on nonlinear filters, IEEE Trans. Pattern Anal. Machine Intelligence 8, 538-550.

Smith S.W., Wagner R.F., Sandrik J.M. and Lopez H. (1983): Low contrast detectability and contrast/detail analysis in medical ultrasound, IEEE Trans. Sonics Ultrasonics 30, 164-173. 
Swets J.A. and Pickett R.M. (1982): Evaluation of Diagnostic Systems (Academic Press, New York).

Thijssen J.M., Oosterveld B.J. and Wagner R.F. (1988): Gray level transforms and lesion detectability in echographic images, Ultrasonic Imaging 10, 171-195.

Trahey G.E., Allison J.W., Smith S.W. and Von Ramm O.T. (1986): A quantitative approach to speckle reduction via frequency compounding, Ultrasonic Imaging 8, 151-164.

Verhoeven J.T.M. and Thijssen J.M. (1993): Improvement of lesion detectability by speckle reduction filtering: a quantitative study, Ultrasonic Imaging 15, 181-204.

Wagner R.F. (1986): Fundamentals and applications of signal detection theory in medical imaging, in Proc. SPIE 626, pp. 755-761.

Wagner R.F. and Brown D.G. (1985): Unified SNR analysis of medical imaging systems, Phys. Med. Biol. 30, 489-518.

Wagner R.F., Smith S.W., Sandrik J.M. and Lopez H. (1983): Statistics of speckle in ultrasound Bscans, IEEE Trans. Sonics Ultrasonics 30, 156-163.

\section{Appendix A}

Let $O^{\prime}(y)$ be the number of discrete points covered by the window produced by amplitude point $A(y)$. It follows that

$$
\bar{N}=\lim _{n \rightarrow \infty} \frac{\sum_{y=-n}^{+n} O^{\prime}(y)}{2 n+1}=\overline{O^{\prime}}
$$

A difference exists between the local window size $O(y)$, and the number of points covered by the same window, $O^{\prime}(y)$, which is always an integer number. The discrepancy results from the discrete character of the amplitude distribution $A$. If $O(y)$ $=2 d_{y}$ then $O^{\prime}(y)=2$ (int) $d_{y}+1$, where (int) $d_{y}$ indicates the integer value $d_{y} . O^{\prime}(y)$ can both be smaller and larger than $O(y)$. The minimum value $O^{\prime}(y)$ can assume is one. It will be demonstrated that $\overline{O^{\prime}} \approx \bar{O}$, despite the local differences between $O^{\prime}(y)$ and $O(y)$, so that also $\bar{N} \approx \bar{O}$.

Variable (int) $d_{y}$ can be expressed as:

$$
\text { (int) } d_{y}=d_{y}-\rho\left(d_{y}\right)
$$

where $\rho\left(d_{y}\right)$ is the decimal part of $d\left(0 \leq \rho\left(d_{y}\right)<1\right)$. No linear relationship exists be- 
tween $\rho\left(d_{y}\right)$ and $d_{y}$. In practice, $\rho\left(d_{y}\right)$ will be uniformly distributed over the interval $[0,1)$, so that $\overline{\rho\left(d_{y}\right)} \approx 0.5$. Using Eq. (5.A2), the expression for $\overline{O^{\prime}}$ becomes:

$$
\begin{aligned}
\overline{O^{\prime}} & =\overline{\left(2(\text { int }) d_{y}+1\right)} \\
& =\overline{2\left(d_{y}-\rho\left(d_{y}\right)\right)+1} \\
& =2 \overline{d_{y}}-2 \overline{\rho\left(d_{y}\right)}+1 \\
& \approx 2 \overline{d_{y}}=\bar{O}
\end{aligned}
$$

Even if $\rho\left(d_{y}\right)$ is not uniformly distributed over $[0,1)$, the absolute difference between $\bar{O}$ and $\bar{O}^{\prime}$ is always smaller than 1 . The relative difference $/ \overline{O^{\prime}}-\bar{O} / / \bar{O}$ decreases with increasing $\bar{O}$.

\section{Appendix B}

According to Eq. (5.13), the mean $N$ value is calculated as:

$$
\bar{N}=1+2 \sum_{y=1}^{\infty} \overline{B(y)}= \begin{cases}1+2 \sum_{y=1}^{\infty} e^{-\frac{(2 y / c)^{2 / q}}{2 \psi}} & (q>0) \\ 1+2 \sum_{y=1}^{\infty}\left(1-e^{-\frac{(2 y / c)^{2 / q}}{2 \psi}}\right) & (q<0)\end{cases}
$$

Combining Eq. (5.B1) with Eq. (5.7) yields:

$$
\begin{array}{ll}
2 \sum_{y=1}^{\infty} e^{-\frac{(2 y / c)^{2 / q}}{2 \psi}} \approx c \cdot(2 \psi)^{\frac{q}{2}} \cdot \Gamma\left(\frac{q}{2}+1\right)-1 & (q>0) \\
2 \sum_{y=1}^{\infty}\left(1-e^{-\frac{(2 y / c)^{2 / q}}{2 \psi}}\right) \approx c \cdot(2 \psi)^{\frac{q}{2}} \cdot \Gamma\left(\frac{q}{2}+1\right)-1 & (q<0)
\end{array}
$$

The left terms are exactly equal to $\bar{N}$ while the right terms closely approach $\bar{N}$.

Using the upper part of Eq. (5.B2), $\operatorname{var}(N)$ is calculated for $q>0$ according to: 


$$
\begin{aligned}
\operatorname{var}(N) & \approx 2 L_{N}^{2} \cdot \sum_{n=1}^{\infty}\left(e^{-\frac{\left(2 L_{N} \cdot n / c\right)^{\frac{2}{q}}}{2 \psi}}-e^{-\frac{\left(2 L_{N} \cdot n / c\right)^{\frac{2}{q}}}{\psi}}\right) \\
& \approx L_{N}^{2} \cdot\left\{\left(c / L_{N}\right) \cdot(2 \psi)^{\frac{q}{2}} \cdot \Gamma\left(\frac{q}{2}+1\right)-\left(c / L_{N}\right) \cdot(\psi)^{\frac{q}{2}} \cdot \Gamma\left(\frac{q}{2}+1\right)\right\} \\
& =L_{N} \cdot c \cdot\left((2 \psi)^{\frac{q}{2}}-\psi^{\frac{q}{2}}\right) \cdot \Gamma\left(\frac{q}{2}+1\right) \\
& \approx L_{N} \cdot\left(1-2^{-q / 2}\right) \cdot \bar{N}
\end{aligned}
$$

Similarly, it is found for $q<0$ that $\operatorname{var}(N)=L_{N} \cdot\left(2^{-q / 2}-1\right) \cdot \bar{N}$, yielding the general expression for $\operatorname{var}(N)$ :

$$
\operatorname{var}(N) \approx L_{N} \cdot\left|1-2^{-q / 2}\right| \cdot \bar{N}
$$





\section{6}

\section{Postprocessing of Velocity Distributions in Real-Time Ultrasonic Color Velocity Imaging}

Chapter 6 is based on the publication:

R.J. Collaris and A.P.G. Hoeks (1994): Postprocessing of velocity distributions in real-time ultrasonic color velocity imaging, Ultrasonic Imaging 16, 249-264. 
Chapter 6 


\subsection{Abstract}

A robust processing scheme is proposed that improves the presentation of twodimensional velocity distributions in real-time ultrasonic color velocity images. Essentially, the algorithm is a modification of a first-order recursive filter, processing each velocity signal in the spatial distribution separately from the others. It restores the sudden holes and notches in the velocity profiles that occur wherever the observed blood velocity is incidentally close to zero. At the same time, unlike conventional persistence filters, it does not influence any of the true velocity information that is measured. The result is a consistent sequence of color velocity images with smooth transitions between the borders of the consecutive velocity profiles and with an improved definition of the regions containing blood.

Key words: Persistence filtering; real-time velocity signal processing.

\subsection{Introduction}

Ultrasonic color velocity imaging is widely used in clinical applications to obtain real-time two-dimensional blood velocity distributions. Generally, two classes of velocity assessment techniques are distinguished. One class contains conventional Doppler shift techniques (Barber et al., 1985; Kasai et al., 1985; Van Leeuwen et al., 1986) while the other is based on more recently developed rf cross correlation techniques (De Jong et al., 1990; Foster et al., 1990; Hein et al., 1993; Hein and O'Brien Jr., 1993). In both classes, the instantaneous velocity distributions that are obtained have some shortcomings, which are especially annoying when observed in rapid succession, as in real-time applications. The origin and extent of these shortcomings can be explained as follows.

A color velocity image is a superposition of a gray level echo image displaying structural information and a color encoded velocity distribution showing the instantaneous blood velocities. A pixel in the image represents either structural information or blood velocity information, but not both at the same time. The general rule is that in every location in the image for which a valid blood velocity has been measured, this information is also displayed, while the structural information is displayed everywhere else. However, it is impossible to tell beforehand where moving blood is situated in the image. That is why a decision criterion has to be applied for the recognition of relevant blood velocity information. For this purpose, local values of signal parameters, like the Doppler signal amplitude (Kerr and Hunt, 1992), the Doppler signal bandwidth, the signal-to-noise ratio and the velocity estimate itself 
are compared with a threshold. If the condition is not satisfied, the corresponding velocity estimates are rejected.

Two difficulties arise here. In the first place, the stochastic character of the underlying rf signals causes the parameters to show some random fluctuations, which lead to misrecognitions occurring everywhere in the image, but especially at those locations where the velocities are low and the parameter values tend to be close to the threshold. This often happens at the border of the lumen, near the vessel wall. Such failures lead to a color velocity map with noise spots outside the lumen (false positives), with small holes within the blood velocity profile (false negatives) and with borders of velocity profiles having a rather frayed appearance. The second difficulty stems from the necessity to apply a high pass filter to remove the stationary tissue component in the signals, which makes it impossible to assess the lowest blood velocities. Consequently, all estimates close to zero are rejected, as they are an indication of leakage of the high pass filter. As a result, large notches and holes may show up at the border of the velocity profile, and, depending on the character of the blood flow and occurrence of secondary echoes, also in the middle of the lumen. The most annoying aspect is that in a subsequent frame, the shapes and positions of the large gaps will be different (especially if the blood flow changes rapidly in time, as in the aorta). Accordingly, the spot noise pattern as well as the frayed border pattern will have changed randomly.

The consequence of all this is, in the first place, that the locations where the blood is situated seem ill-defined and, secondly, since the human visual system is very sensitive to rapid fluctuations, that the clinician might be distracted in realtime applications, where consecutive images follow each other at high rate. Of course, the transfer of information will then be far from optimal.

Clearly, effort is required for improvement. As regards the single false negatives and positives, fewer mistakes will be made if more time is spent on the measurement of a velocity distribution, as this will enable a more accurate calculation of the local signal parameter values. However, since the frame rate decreases proportionally, this is at the expense of the time resolution of blood velocities. Moreover, the lower the frame rate becomes, the more the intended movie effect disappears and the more the observer will perceive the images separately. A more effective way to cope with these shortcomings in the color velocity images is the application of signal postprocessing techniques. Spatial median filtering of the velocity distribution with a sliding kernel, for example, is a powerful means for removing the small holes as well as the outliers. However, it is not a remedy against the large holes in the velocity profiles and the random jitter of the borders. Obviously, time processing of con- 
secutive frames is necessary to achieve actual improvement.

In this paper, we present a robust, real-time processing scheme that produces a considerably more consistent sequence of color velocity images with smoother transitions between the consecutive borders of the velocity distributions, a better definition of the regions containing blood and an improved presentation of the low velocities. The algorithm is especially suitable for use under dynamic flow conditions. Most importantly, it achieves actual improvement while affecting the true velocity information available as little as possible.

\subsection{Assumptions}

If the assessment of the local instantaneous blood velocity is based on a correlation search algorithm, in principle, no upper limit exists to the maximum detectable velocity (Jensen, 1993). In practice however, the range of search is often restricted to half a period of the carrier frequency, limiting the maximum detectable velocity to $\left|v_{\max }\right|=0.25 \lambda$.PRF, where $\lambda$ is the mean wavelength of ultrasound in tissue and $P R F$ is the pulse repetition frequency. Evidently, this value is equal to the maximum velocity unambiguously assessable with Doppler signal processing. The Nyquist theorem confines the Doppler frequency $f_{d}$ to the interval $-0.5 P R F \ldots+0.5 P R F$ and, using the Doppler equation, also limits the velocity to $\left|v_{\max }\right|=0.5 \lambda \cdot\left|f_{d, \max }\right|=$ $0.25 \lambda \cdot P R F$. All theory becomes independent of the system parameters if velocity $v$ is expressed in units of $\lambda \cdot\left|f_{d, \max }\right|$, which yields: $\left|v_{\max }\right|=0.5$. On the other hand, a lower limit also exists for the velocities estimated, invoked by the high pass filter used to remove the tissue contribution to the signals. Let this limit $T_{v}$ also be expressed as a fraction of $\lambda \cdot\left|f_{d \text { max }}\right|$. It is then assumed that all velocity estimates with $|V|<T_{V}$ have been set to zero, so that $\left|v_{\min }\right|=T_{v}$.

In color velocity imaging, $v$ is a function of both location and time, so we will write $v$ as $v_{t}(i, j)$, to emphasize the spatial character of the distribution, or as $v_{i, j}(t)$, to stress the temporal behavior of each velocity signal in the spatial distribution. The coordinates $t, i$ and $j$ denote time (i.e., frame number), scan position (i.e., scan line) and sample volume, respectively. Note that this formulation makes no distinction between linear array and sector scanning systems.

Once the false estimates in the velocity distribution $v_{t}(i, j)$ have been rejected, a scan converter performs spatial interpolation to transform $v_{t}(i, j)$ into the image distribution $v_{t}^{\prime}(x, y)$, where $x$ and $y$ indicate location in a rectangular grid of pixels. The final color velocity image at time $t$ is obtained by mixing the velocity distribution 
$v_{t}^{\prime}(x, y)$ with the accompanying echo image. Generally, the two distributions are joined in such a way that a velocity pixel is displayed if $\left|v_{t}^{\prime}(x, y)\right|>T_{d}$, while the echo pixel is displayed otherwise. Thus, the threshold $T_{d}$ determines the lowest velocity value that may be presented in the color velocity image. Normally, $T_{d}$ equals $T_{v}$, but it will be shown that in the application of the algorithm to be presented, it makes sense to choose $T_{d}<T_{v}$.

In short, it will be assumed that:

- velocities are normalized with respect to $\lambda \cdot\left|f_{d, \max }\right|$, so that $\left|v_{\max }\right|=0.5$.

- in the algorithm's input velocity distribution, velocity estimates within the range $-T_{v} \ldots+T_{v}$ are set to zero. Threshold $T_{v}$ is equal to a small fraction of the full scale. Thus $\left|v_{\min }\right|=T_{v}$.

- the false positives in $v_{t}(i, j)$ have been rejected by setting them to zero, so that only actual blood velocity information is present.

\subsection{Description of the algorithm}

The unrest perceived in the color velocity movie is not so much caused by rapid color changes, whether reflecting actual velocity fluctuations or just being due to the variance in the velocity estimation, but rather by the rapid alterations in the velocity profile borders over consecutive frames. Hence, the main effort is directed at the reduction of border jittering and the filling of gaps, while affecting the actual velocity information as little as possible.

The algorithm we propose can be applied to the distribution $v_{i, j}(t)$ as well as to $v_{x, y}^{\prime}(t)$, although the latter is preferred, as will be discussed later. It performs nonlinear time filtering of each velocity signal in the spatial distribution. As this is essentially a one-dimensional process, which is repeated for every location in the plane of observation, we will leave out the space coordinates. The algorithm will be presented as a modification in two steps of the attack-sustain filter described by:

$$
\begin{aligned}
& \text { if }\left|v_{\text {in }}(t)\right|>\left|v_{\text {out }}(t-1)\right| \text { or } \operatorname{sgn}\left(v_{\text {in }}(t)\right)==-\operatorname{sgn}\left(v_{\text {out }}(t-1)\right) \\
& \left\{\quad v_{\text {out }}(t)=v_{\text {in }}(t) ;\right. \\
& \} \\
& \text { else } \\
& \left\{\quad v_{\text {out }}(t)=v_{\text {out }}(t-1)+c \cdot\left(v_{\text {in }}(t)-v_{\text {out }}(t-1)\right) ;\right. \\
& \}
\end{aligned}
$$


where $v_{\text {in }}(t)$ and $v_{\text {out }}(t)$ are the algorithm's input and output at time $t$, respectively, and $\operatorname{sgn}(v)$ is the sign operator, which returns 1 if $v>0,-1$ if $v<0$ and 0 if $v=0$. Parameter $c(0<c<1)$ is related to the response time of the first-order recursive filter described in Eq. (6.1b) according to:

$$
\tau_{\text {resp. }}=-\frac{1}{\ln (1-c)}
$$

The attack-sustain filter makes a distinction between two situations. If the velocity signal progresses in an increasing way (systolic phase), or if a sudden reversal in velocity direction is encountered, the original input signal is presented (attacking state, Eq. (6.1a)). If, on the other hand, the velocity signal progresses in a decreasing way (diastolic phase), a low pass filter is applied (sustaining state, Eq. (6.1b)). Essentially, the two states that are distinguished express a compromise between the preservation of velocity dynamics and the improvement of the consistency between consecutive velocity profiles. The attack-sustain filter combines a presentation of unfiltered peak velocity values (which are indispensable in a reliable presentation of flow events) with the filling of sudden holes and notches in the velocity profiles. The character of the compromise is determined by the filter parameter $c$. The closer $c$ gets to 0 , the more reduction of border jittering is favored over truthful presentation of rapidly decreasing velocity signals, and vice versa. The delicacy of the adjustment of $c$ might be illustrated by the visibility of the carotid notch (a dip in the carotid blood flow in the postsystolic phase), which strongly depends on the precise choice of $c$.

The modifications to be made are intended to transform the compromise into an optimal behavior in all situations. As a useful tool for showing the effects of the modifications, the filter's behavior is presented in figure 6.1 as a plane in the threedimensional space constituted by the positive variables $v_{\text {in }}(t), v_{\text {out }}(t-1)$ and $v_{\text {out }}(t)$. The dark gray plane represents the output $v_{\text {out }}(t)$ for those combinations of $v_{i n}(t)$ and $v_{\text {out }}(t-1)$ for which is true that $v_{\text {in }}(t) \leq v_{\text {out }}(t-1)$ (other combinations are not considered as the algorithm only filters decreasing signal segments). It should be kept in mind that, although the plane, being solely a property of the filter, is also defined for $0<v_{\text {in }}(t)<T_{v}$, these values never occur in the input signal. In the plane $v_{i n}(t)=0$ (not explicitly drawn), the ratio $v_{\text {out }}(t) / v_{\text {out }}(t-1)$ is a constant and equals $\tan (\theta)=1-c$. If $c$ is equal to one, no filtering is performed at all and the dark gray plane coincides with the light gray plane showing all points with $v_{\text {out }}(t)=v_{\text {in }}(t)$.

\subsubsection{Modification 1}

The first modification consists in making parameter $c$ a function of the modu- 


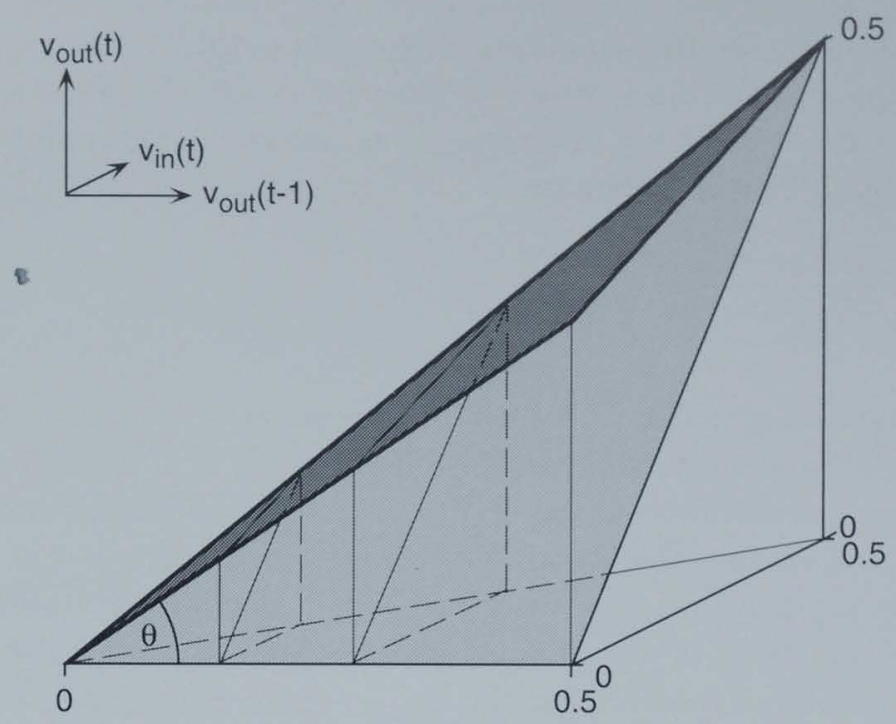

Figure 6.1. Description of a linear first-order recursive filter by a plane (dark gray) in the three-dimensional space constituted by the variables $v_{\text {in }}(t), v_{\text {out }}(t-1)$ and $v_{\text {out }}(t)$.

lus of the latest output of the algorithm:

$$
c=c\left(\left|v_{\text {out }}(t-1)\right|\right)
$$

This causes the filter to exhibit a nonlinear behavior. The idea behind a variable $c$ is that the rapid changes in velocity that actually attract the attention of the observer always occur at high velocities. Hence, for minimal signal affection, the filter should have a very short response time for high velocities and, consequently, $c$ should be close to 1 . On the other hand, as was discussed above, the jittering of the velocity profiles is essentially a low velocity process, in which blood velocities fluctuate around the threshold $T_{V}$ below which velocity estimation fails. The sequence of velocity profiles would become much more consistent if a sudden drop in the local blood velocity below $T_{v}$ were handled by forcing the corresponding displayed velocity to sustain for a while in the vicinity of $T_{v}$. Thus, as an immediate reaction to the incidental vanishing of the local blood velocity information, the algorithm should present a low velocity value that slowly decays over the next few frames until it eventually drops below the threshold $T_{d}$, marking the lower boundary of the velocities displayed. This effect is obtained by giving the filter a long response time (corresponding to a small $c$ ) for low velocities. 
How should one define the exact shape of the $c$-function? Figure 6.2 reflects the idea of sustaining low velocities in the neighborhood of $T_{v}$. For every possible output value of the algorithm at time $t-1$, the bold curve represents the output at time $t$ in response to a sudden jump to zero of the input signal. If Eq. (6.1b) is combined with Eq. (6.3), it follows that the bold curve is described by:

$$
v_{\text {out }}(t)=\left(1-c\left(\left|v_{\text {out }}(t-1)\right|\right)\right) \cdot v_{\text {out }}(t-1)
$$

Obviously, the sudden jump to zero of the input signal does not lead to a similar jump to zero of the output signal. Instead, the output $v_{\text {out }}(t)$ assumes a level slightly higher than $T_{v}$. If the input signal remains zero for several frames, the progress of the output signal can be found by carrying out the iterative process also shown in figure 6.2. The row of dots on the horizontal axis represents an example of a possible sequence of velocities. As soon as the velocity value indicated by the rightmost dot is displayed, the input signal drops down to zero. In response, the output in the next frame assumes the level of the bold curve above the dot. This level is projected, via the line $v_{\text {out }}(t)=v_{\text {out }}(t-1)$, on the horizontal axis (see the next rightmost dot). In its

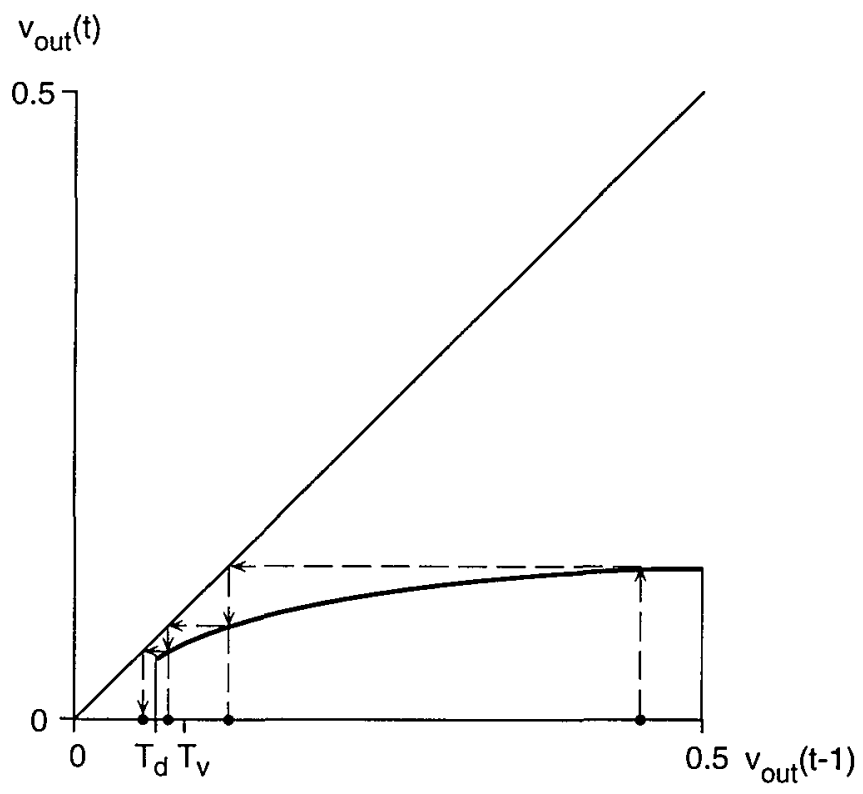

Figure 6.2. Curve describing the relation between $v_{\text {out }}(t-1)$ and $v_{\text {out }}(t)$ for $v_{\text {in }}(t)=0$. The progress of $v_{\text {out }}(t)$ (represented by the row of dots) is obtained by carrying out the iterative process indicated by the arrows. 
turn, this velocity value serves as the input for the following frame in the sequence, and so on. The process stops when the velocity level has reached the threshold $T_{d}$ (see the leftmost dot). Thus, the number of frames during which the low-velocity signal persists equals the number of iteration steps necessary to reach the level $T_{d}$. Of course, the whole process is prematurely terminated if relevant velocity information becomes available once again to be displayed. Note that although the velocities measured range between $T_{v}$ and 0.5 , the sustaining action allows the velocities displayed to drop below $T_{v}$, down to $T_{d}\left(0<T_{d}<T_{v}\right)$. Thus, the iteration path is extended towards zero, yielding the opportunity to achieve equal persistence (in number of frames) with a shorter filter response time for low velocities. The result is that sudden notches and holes in the profiles are filled with velocity values quickly progressing towards a level close to zero. The effect is an improved reflection of flow reality, with the borders of velocity profiles indicating the actual boundaries of blood-containing structures and with the lower limit $T_{v}$ apparently shifted downwards to zero.

An expression of the $c$-function is obtained on the basis of the bold curve in figure 6.2. A possible mathematical description of this curve for both positive and negative velocities is given by:

$$
v_{\text {out }}(t)=u\left(v_{\text {out }}(t-1)\right)=\left(\gamma_{2} \cdot e^{-\gamma_{1} \cdot v_{\text {out }}(t-1) \mid}+\gamma_{3}\right) \cdot \operatorname{sgn}\left(v_{\text {out }}(t-1)\right) \quad\left(\gamma_{2}<0\right)
$$

where $\gamma_{1}, \gamma_{2}$ and $\gamma_{3}$ are parameters determining the exact shape of the curve, indicated by $u\left(v_{\text {out }}(t-1)\right)$. It should be realized that this is certainly not the only possible description. Other approximations, e.g. based on the arc tangent, will also work. Combining Eq. (6.4) and Eq. (6.5) yields:

$$
c\left(\left|v_{\text {out }}(t-1)\right|\right)=1-\frac{u\left(v_{\text {out }}(t-1)\right)}{v_{\text {out }}(t-1)}=1-\frac{\gamma_{2} \cdot e^{-\gamma_{1}\left|v_{\text {out }}(t-1)\right|}+\gamma_{3}}{\left|v_{\text {out }}(t-1)\right|}
$$

For practical reasons, $\gamma_{2}$ and $\gamma_{3}$ are not directly substituted. Instead, they are expressed in the more easily interpretable variables $\gamma_{1}, u\left(T_{d}\right)$ and $u(0.5)$, as described in the Appendix. $u\left(T_{d}\right)$ and $u(0.5)$ define the beginning and end of the $u$-curve. Figure 6.2 shows that the ratio $u\left(v_{\text {out }}(t-1)\right) / v_{\text {out }}(t-1)$ is low for values of $v_{\text {out }}(t-1)$ close to 0.5 , which indeed leads, via Eq. (6.4), to $c$-values close to 1 . In the same way, $c$-values become lower for $v_{\text {out }}(t-1)$ approaching $T_{d}$. Figure 6.3-a illustrates how changing $\gamma_{1}$ influences the $u$-curve, while figure 6.3 -b shows the effect on the corresponding $c$-characteristic. 

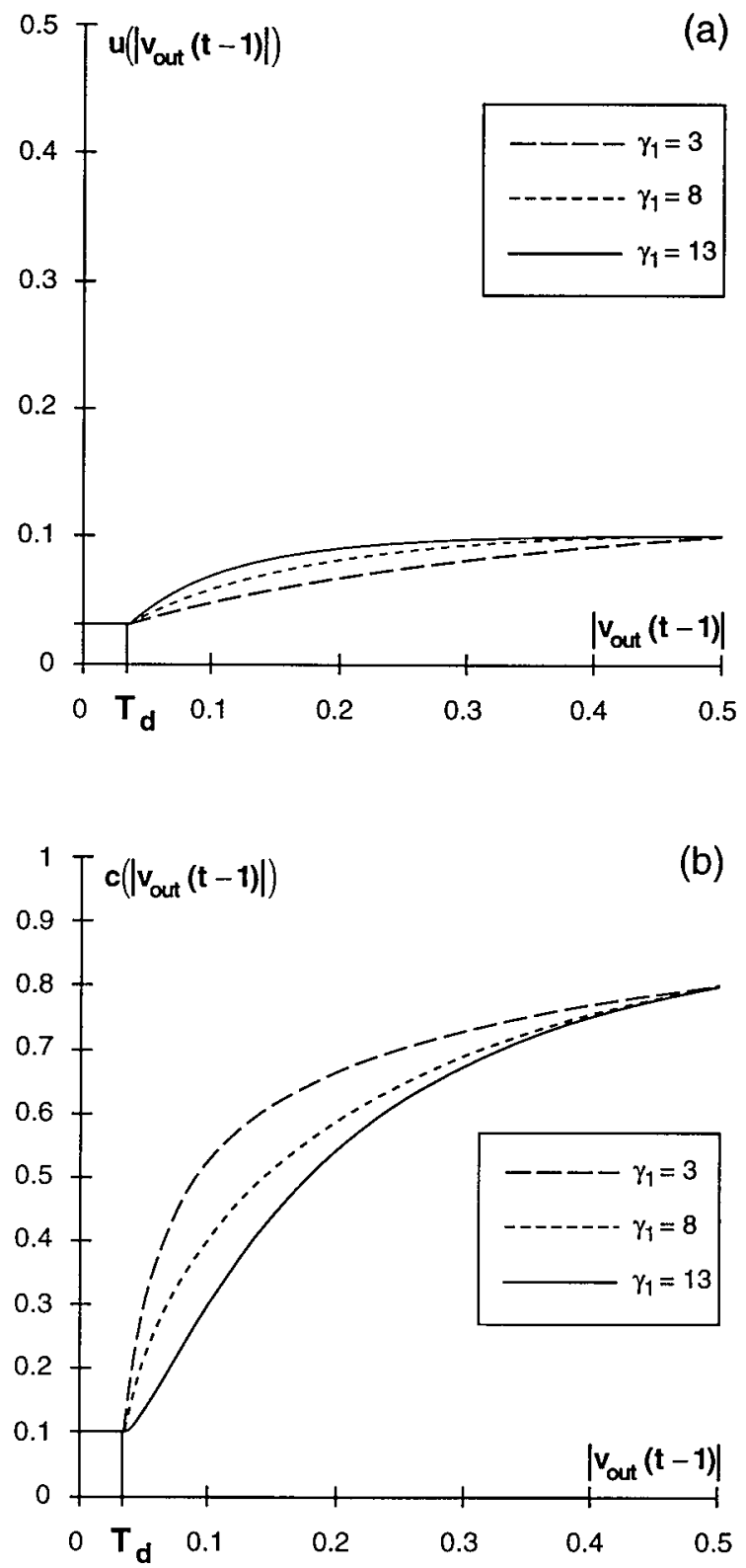

Figure 6.3. (a) Three $u$-curves for different values of parameter $\gamma_{1}$. (b) The $c$-characteristics corresponding to the $u$-curves of (a). 


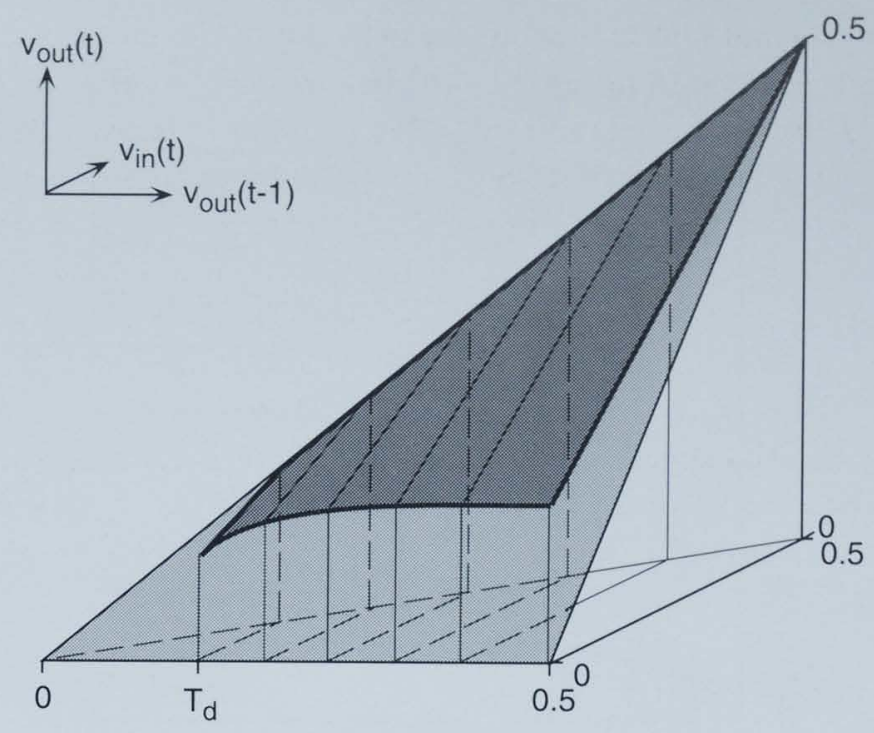

Figure 6.4. Description of the algorithm after modification 1 by a curved surface (dark gray) in the three-dimensional space constituted by the variables $v_{\text {in }}(t), v_{\text {out }}(t-1)$ and $v_{\text {out }}(t)$.

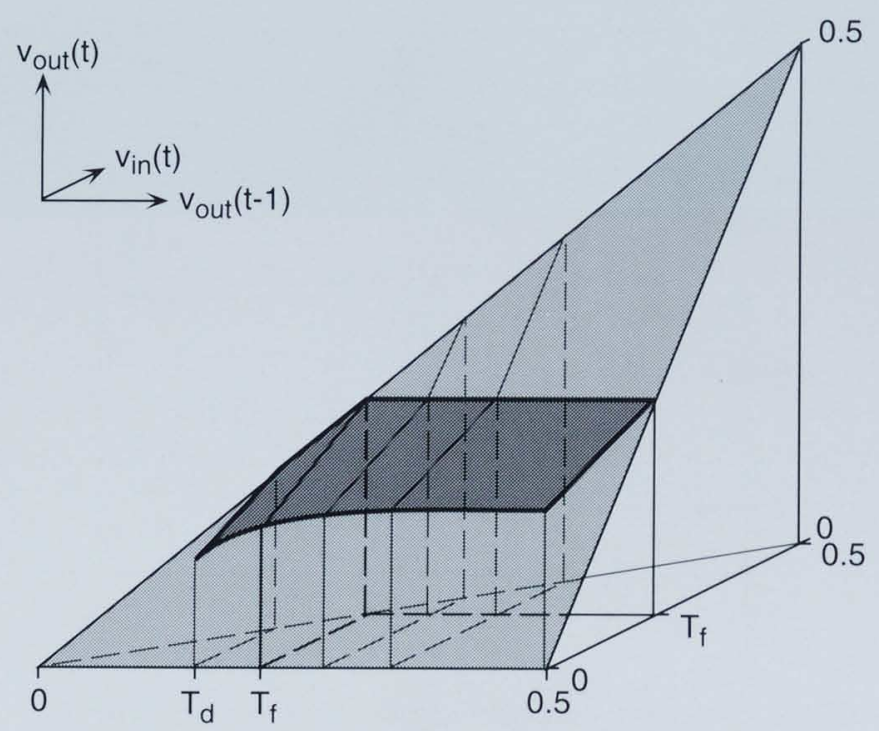

Figure 6.5. Description of the algorithm after modification 2 by a curved surface (dark gray) in the three-dimensional space constituted by the variables $v_{\text {in }}(t), v_{\text {out }}(t-1)$ and $v_{\text {out }}(t)$. 
A three-dimensional plot indicating the behavior of the nonlinear filter for positive velocities is presented in figure 6.4 , which shows this filter to be described by a curved surface (dark gray) instead of the flat plane defining the linear first-order recursive filter. Since the $u$-curve in the plane $v_{i n}(t)=0$ (which was already depicted in figure 6.2) is only defined for $v_{\text {out }}(t-1)>T_{d}$, the curved surface too is restricted to values of $v_{\text {out }}(t-1)$ larger than $T_{d}$. The straight lines of constant $v_{\text {out }}(t-1)$ on the surface have slopes which are equal to $c\left(v_{\text {out }}(t-1)\right)$. They are rather steep for values of $v_{\text {out }}(t-1)$ close to 0.5 , corresponding to short response times, and they become flatter as $v_{\text {out }}(t-1)$ decreases, expressing longer response times for low values of $v_{\text {out }}(t-1)$. Note that the curved surface, like the plane describing the linear recursive filter, descends for both decreasing $v_{i n}(t)$ and decreasing $v_{\text {out }}(t-1)$. This important property means that the filter preserves the larger-than-smaller-than order in the velocity signals.

\subsubsection{Modification 2}

It will be demonstrated that yet another modification is possible, which makes the filter exhibit an even faster response for the majority of velocities, while leaving its capability to fill holes and suppress border jittering unaffected. It may be argued that since high velocities are not involved in the process of jittering, the filter only needs to be active at low values of $v_{i n}(t)$. However, if the filter were switched on suddenly upon $v_{i n}(t)$ dropping below a certain level, the rule of order preservation would be violated, resulting in an inconsistent velocity distribution. This effect is avoided if the filter is turned on gradually, as depicted in figure 6.5. Once again, the dark gray surface describes the filter's behavior. It can be seen that the filter is not used unless $v_{i n}(t)$ drops to below level $T_{f}$. For values of $v_{i n}(t)$ just below this threshold, the filter has only a subtle effect, but its action becomes stronger as $v_{\text {in }}(t)$ decreases to zero, finally resulting in the desired sustaining of low velocities. The $u$-curve describing the relationship between $v_{\text {out }}(t)$ and $v_{\text {out }}(t-1)$ for $v_{i n}(t)=0$ has not been changed as compared with figure 6.4. Consequently, suppression of border jittering has not altered. The equations defining the dark gray surface $S$ for both positive and negative velocities are:

$S\left(v_{\text {in }}(t), v_{\text {out }}(t-1)\right)= \begin{cases}S_{1}\left(v_{\text {in }}(t), v_{\text {out }}(t-1)\right) & \text { for }\left|v_{\text {out }}(t-1)\right| \leq T_{f} \\ S_{2}\left(v_{\text {in }}(t), v_{\text {out }}(t-1)\right) & \text { otherwise }\end{cases}$

with 
$S_{1}\left(v_{\text {in }}(t), v_{\text {out }}(t-1)\right)=v_{\text {in }}(t)+\left(1-\frac{\left|v_{\text {in }}(t)\right|}{\left|v_{\text {out }}(t-1)\right|}\right) \cdot u\left(v_{\text {out }}(t-1)\right)$

and

$S_{2}\left(v_{\text {in }}(t), v_{\text {out }}(t-1)\right)=v_{\text {in }}(t)+\left(1-\frac{\left|v_{\text {in }}(t)\right|}{T_{f}}\right) \cdot u\left(v_{\text {out }}(t-1)\right)$

with

$$
\mathrm{u}\left(\mathrm{v}_{\text {out }}(\mathrm{t}-1)\right)=\left(\gamma_{2} \mathrm{e}^{-\gamma_{1}\left|\mathrm{v}_{\text {out }}(t-1)\right|}+\gamma_{3}\right) \cdot \operatorname{sgn}\left(\mathrm{v}_{\text {out }}(\mathrm{t}-1)\right)
$$

As can be seen in figure 6.5, the level $T_{f}$ should always be higher than the $u$-curve in the plane $v_{\text {in }}(t)=0$. That is:

$$
T_{f}>u(0.5)
$$

The surface $S$ is incorporated into the algorithm for border jittering suppression as follows:

$$
\begin{aligned}
& \text { if }\left|v_{\text {in }}(t)\right|>\left|v_{\text {out }}(t-1)\right| \text { or }\left|v_{\text {in }}(t)\right|>T_{f} \text { or } \operatorname{sgn}\left(v_{\text {in }}(t)\right)==-\operatorname{sgn}\left(v_{\text {out }}(t-1)\right) \\
& \text { \{ } \\
& v_{\text {out }}(t)=v_{\text {in }}(t) \\
& \text { \} } \\
& \text { else } \\
& \text { \{ } \\
& v_{\text {out }}(t)=S\left(v_{\text {in }}(t), v_{\text {out }}(t-1)\right) \\
& 1
\end{aligned}
$$

Observe that for $\left|v_{\text {out }}(t-1)\right| \leq T_{f}$, this algorithm is identical to the algorithm of Eq. (6.1), with a variable parameter $c$ (rewriting Eq. (6.7b) will reveal this).

\subsection{Application to artificial and real velocity signals}

As this study is concerned with improved presentation of instantaneous velocity information, visual inspection of (pseudo) real-time color velocity images plays a key role in the evaluation of the processing scheme proposed. In an attempt to overcome the limitations of a paper in this respect, the algorithm's ability to sustain low 
velocities with minimal signal affection is illustrated on the basis of a single isolated velocity signal. Subsequently, the spatial effects are highlighted using a number of successive images taken from a real-time sequence.

Figure 6.6 shows a simulated input velocity signal, indicated by the open dots. This signal was derived from a sine-like wave, starting at $t=3$, by setting all velocities smaller than $T_{v}=0.1$ to zero. The sine-like wave has a peak value of 0.5 and a trough value that equals 0.15 at the first trough, and zero at the next two. One period consists of approximately 8 sample points. The algorithm, adjusted with $T_{d}=$ $0.05, T_{v}=0.1, u\left(T_{d}\right)=0.045, u(0.5)=0.12, T_{f}=u(0.5)=0.12$ and $\gamma_{f}=9.0$, produced the output signal represented by the black dots. Clearly, the output follows the input signal exactly for most of the time. Only for values of the input below $T_{f}$ does the algorithm exhibit its sustaining action, preventing the output from dropping to zero. Apparently, the $u$-curve was adjusted in such a way that the output signal could just bridge the periods of zero input (that is, the output reached level $T_{d}$ just when new velocity information became available). It is exactly this sustaining effect that yields a considerably improved definition of the regions containing blood and a better presentation of the lowest velocities in the two-dimensional, spatial situation.

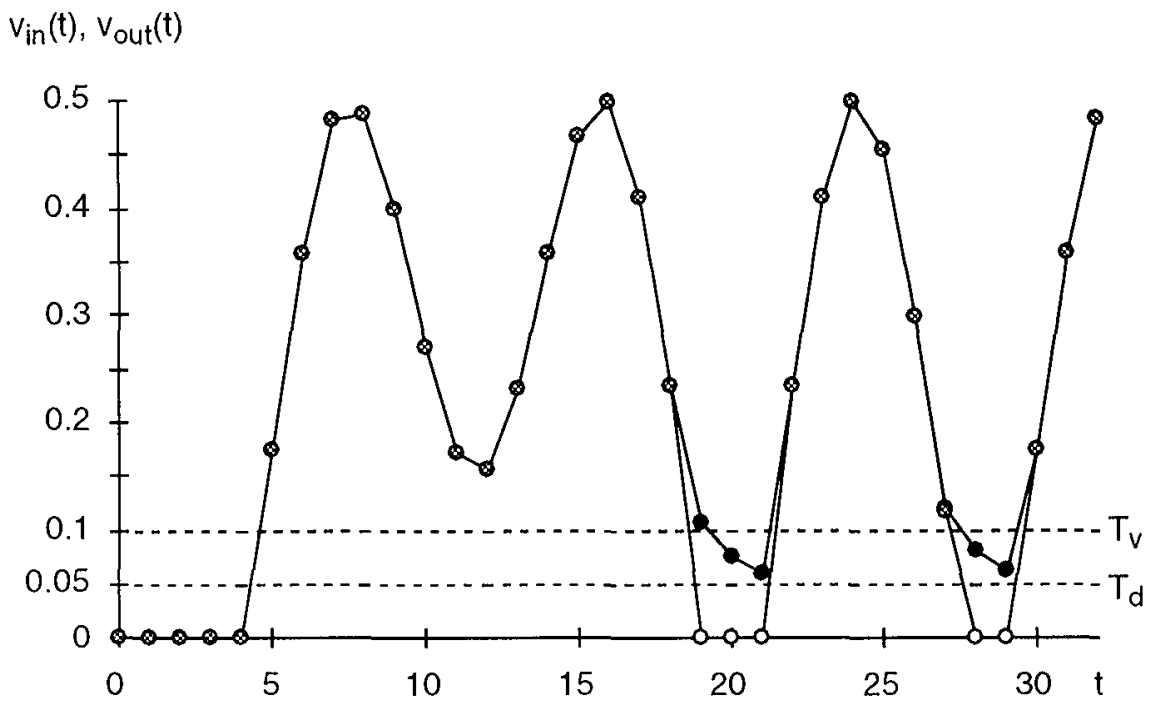

Figure 6.6. Response of the algorithm (black dots) to an artificial input velocity signal (white dots). Coinciding black and white dots are gray. The algorithm is adjusted according to: $T_{d}=$ $0.5, T_{V}=0.05, u\left(T_{d}\right)=0.045, u(0.5)=0.12, T_{f}=u(0.5)=0.12$ and $\gamma_{1}=9.0$. 


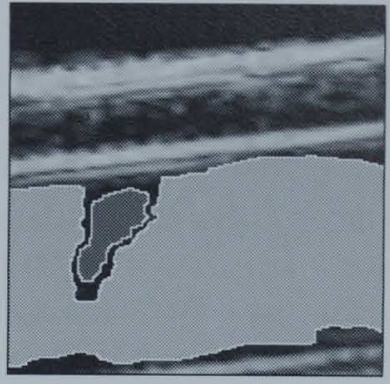

(a)

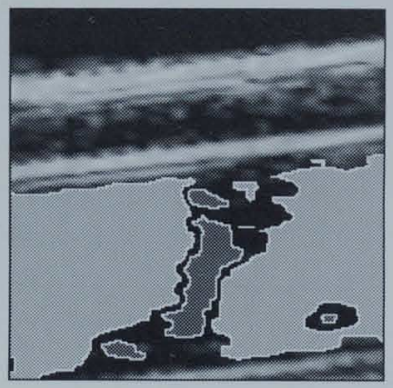

(b)

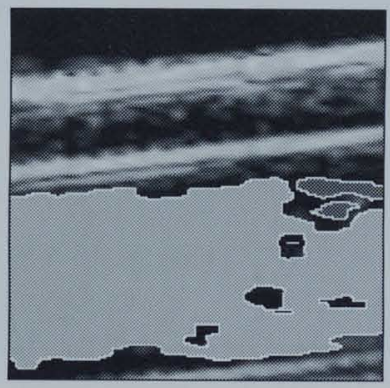

(c)

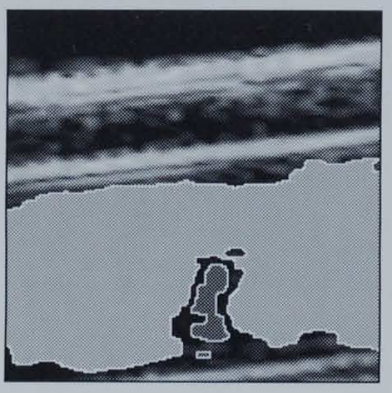

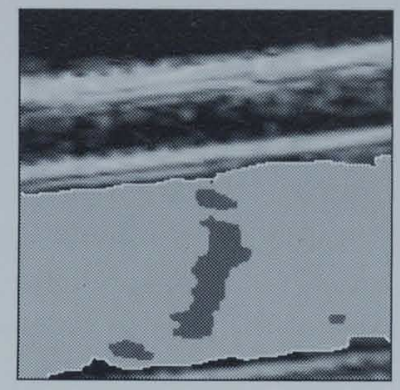
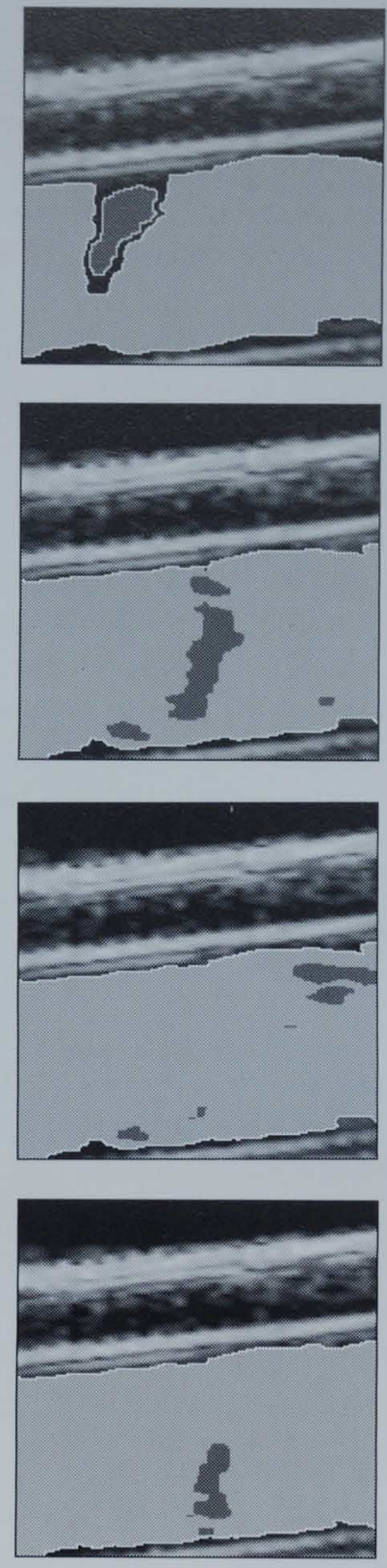

Figure 6.7. A sequence of echo/Doppler images showing a flow phantom in longitudinal view. Echo information is encoded in gray levels, velocity information is encoded in uniform light gray (positive direction) and uniform dark gray (negative direction). The velocity profiles have white boundaries. The left-hand images are the originals, the right-hand images are the corresponding processed ones. (a) frame 1 (left-hand and right-hand image are identical). (b) frame 2. (c) frame 3. (d) frame 4. 
To demonstrate this, measurements were carried out on a flow phantom, consisting of a silicone tube filled with a blood-mimicking fluid (Nutricia Choc) and an electric pump generating a steady mean flow. A narrowing of the tube at one point yielded a locally rapidly fluctuating flow pattern (in space as well as in time), which was registered in longitudinal view by a 32 scan lines $6.1 \mathrm{MHz}$ linear array transducer. A total number of 16 successive frames were recorded at a PRF of $8.1 \mathrm{kHz}$. Each frame contained $16 \mathrm{rf}$ lines per scan position, so that all frames together covered a time interval of approximately one second. All signals were digitized and processed off-line on a PC. Velocity distributions were obtained by standard Doppler processing, false estimates being removed by thresholding the Doppler signal bandwidth, followed by median filtering of the spatial velocity distributions with a $3 \times 3$ kernel. Finally, linear scan conversion was used to transform the velocity information of each frame into a rectangular grid of $200 \times 200$ pixels. The resulting distributions were successively presented to the algorithm. Figures 6.7-a to 6.7-d show the first four frames in the sequence of sixteen (originals on the left, processed ones on the right). For the sake of clarity, the velocity profiles have white edges and they only indicate velocity direction: light gray indicates velocity towards the transducer, while dark gray corresponds to velocity away from the probe. Of course, differences in velocity normally find expression in color gradations, but for the purposes of showing the algorithm's influence on the spatial shape of the profiles, the absence of detailed velocity information is no obstacle. The images cover an area of approximately $2 \times 2 \mathrm{~cm}^{2}$. The location of the narrowing in the tube is just outside the region insonified, to the left of the images. Main blood flow is from left to right. As can be seen in the original images, clear differences exist between the borders of the four profiles close to the anterior and posterior tube walls, and holes appear wherever velocity reverses its direction. In comparison, the sequence of processed images is more consistent. The profiles are continuous (except for the first frame, which lacks a prehistory) and the fluid-containing region is more clearly defined. Since only velocity direction is displayed, the minimal signal affection is only expressed by the fact that sudden velocity reversal is not affected in the processed images.

\subsection{Discussion}

A common characteristic of all signal processing techniques applied in color velocity imaging is the ability to operate under real-time conditions. Although microprocessor speed has increased significantly over the last few years, time limitations still restrict the number of operations that can be carried out. In the development of the present algorithm, real-time applicability has been a major requirement. To keep implementation in hardware relatively simple, the algorithm has been based 
on an ordinary one-dimensional first-order recursive filter, requiring only one frame of velocity and echo data to be saved in the memory. As regards processing time, it is suggested to store the surface $S$ of Eq. (6.7) in a look-up table, as finding $v_{\text {out }}(t)$, given a combination of $v_{i n}(t)$ and $v_{\text {out }}(t-1)$, is then reduced to straightforward table indexing, enabling very high speed operation.

It has been stated that the distributions $v_{i, j}(t)$ and $v_{x, y}^{\prime}(t)$ can both serve as input for the algorithm. Because the number of sample volumes per scan line is generally smaller than the number of corresponding pixels in the image, processing $v_{i, j}(t)$ requires less time than processing $v_{x, y}^{\prime}(t)$. However, the use of the latter is still preferred, for two reasons. The first reason follows from the difference in coarseness between the two distributions. Velocity information originating from one sample volume is spread out over a number of pixels via scan conversion, i.e., linear interpolation. Treating these pixels independently and not as a group, as is done if $v_{x, y}^{\prime}(t)$ serves as the input, results in velocity profiles with boundaries that move more smoothly over consecutive frames. Suppose, for example, that velocity information vanishes in a sample volume located at the border of a velocity profile. If $v_{i, j}(t)$ is the input, a number of corresponding velocity pixels in the image will immediately disappear, despite the sustaining action of the algorithm. This is explained by the fact that scan conversion still allows the peripheral pixels associated with the sample volume to drop below $T_{d}$. This is avoided, however, if $v_{x, y}^{\prime}(t)$ is used, since each pixel then follows its own $u$-curve. If velocity information does not return, the velocity pixels are gradually replaced by echo pixels.

The second reason why $v_{x, y}^{\prime}(t)$ is favored is directly related to the process of scan conversion, in which holes appear in the pixel distribution $v_{x, y}^{\prime}(t)$ wherever two neighboring sample volumes have opposite velocity directions. Linear interpolation causes velocity to cross zero somewhere in between the two sample volumes. Consequently, the corresponding velocity pixels are substituted by echo pixels in the composition of the echo/Doppler image. This effect can be observed in the original images of figure 6.7, where the dark and light gray parts of the profiles never touch. Processing $v_{i, j}(t)$ will not remove these gaps, as they are only induced afterwards. However, application of the algorithm after scan conversion does have the desired effect. As can be seen in the processed images of figure 6.7, the positive and negative parts of the distributions join exactly.

Finally, it should be mentioned that if the $u$-curve has been adjusted in such a way that low velocities persist for quite a while, and if the positions of structures in the echo images change rapidly, e.g., due to a movement of the transducer, velocities may be displayed outside the blood-containing regions for a short period of time. 
In fact, the velocity image lags behind the structure image. A possible solution might be quitting the iteration process if sudden changes in local echo amplitude exceed a predefined limit.

\subsection{Conclusion}

A novel velocity signal processing method has been described, whose capacity to improve the consistency in a sequence of color velocity maps equals that of a conventional persistence filter. The advantage of the present algorithm is that, unlike the normal persistence filter, it does not affect the true velocity information that is measured. This means that the velocity signals are not smoothed in time, making the algorithm especially suitable for operation under dynamic flow conditions. Its simplicity and robustness allow real-time application.

\section{REFERENCES}

Barber W.D., Eberhard J.W. and Karr S.G. (1985): A new time domain technique for velocity measurements using Doppler ultrasound, IEEE Trans. Biom. Eng. 32, 213-229.

De Jong P.G.M., Arts T., Hoeks A.P.G. and Reneman R.S. (1990): Determination of tissue motion velocity by correlation interpolation of pulsed ultrasonic echo signals, Ultrasonic Imaging 12, 84-98.

Foster S.G., Embree P.M. and O'Brien Jr. W.D. (1990): Flow velocity profile via time-domain correlation: error analysis and computer simulation, IEEE Trans. Ultrason. Ferro. Freq. Control 37, 164-175.

Hein I.A., Chen J.T., Jenkins W.K. and O'Brien Jr. W.D. (1993): A real-time ultrasound time-domain correlation blood flow meter: Part 1 - theory and design, IEEE Trans. Ultrason. Ferro. Freq. Control 40, 768-775.

Hein I.A. and O'Brien Jr. W.D. (1993): Current time-domain methods for assessing tissue motion by analysis from reflected ultrasound echoes - a review, IEEE Trans. Ultrason. Ferro. Freq. Control 40, 84-102.

Jensen J.A. (1993): Range/velocity limitations for time-domain blood velocity estimation, Ultrasound Med. Biol. 19, 741-749.

Kasai C., Namekawa K., Koyano A. and Omoto R. (1985): Real-time two-dimensional blood flow imaging using an autocorrelation technique, IEEE Trans. Sonics Ultrasonics 32, 458-464.

Kerr A.T. and Hunt J.W. (1992): A method for computer simulation of ultrasound Doppler color flow images - II. Simulation results, Ultrasound Med. Biol. 18, 873-879. 
Van Leeuwen G.H., Hoeks A.P.G. and Reneman R.S. (1986): Simulation of real-time frequency estimators for pulsed Doppler systems, Ultrasonic Imaging 8, 252-271.

\section{Appendix}

The beginning and end of the $u$-curve are defined by $u\left(T_{d}\right)$ and $u(0.5)$, respectively. From Eq. (6.5) it follows that:

$$
u\left(T_{d}\right)=\gamma_{2} \cdot e^{-\gamma_{1} \cdot T_{d}}+\gamma_{3}
$$

and

$$
u(0.5)=\gamma_{2} \cdot e^{-0.5 \gamma_{1}}+\gamma_{3}
$$

Combining Eq. (6.A1) and Eq. (6.A2) yields:

$$
\gamma_{2}=\frac{u\left(T_{d}\right)-u(0.5)}{e^{-T_{d} \cdot \gamma_{1}}-e^{-0.5 \gamma_{1}}}
$$

and

$$
\gamma_{3}=u\left(T_{d}\right)-\gamma_{2} \cdot e^{-\gamma_{1} \cdot T_{d}}
$$

Note that although there is a certain freedom in the choice of the three parameters $\gamma_{1}, u\left(T_{d}\right)$ and $u(0.5)$, the iteration process of figure 6.2 only works out correctly if the curve never exceeds the line $v_{\text {out }}(t)=v_{\text {out }}(t-1)$. Since

$$
u^{\prime}\left(v_{\text {out }}(t-1)\right)=-\gamma_{1} \cdot \gamma_{2} \cdot e^{-\gamma_{1}\left|v_{\text {out }}(t-1)\right|} \cdot \operatorname{sgn}\left(v_{\text {out }}(t-1)\right) \quad\left(\gamma_{2}<0\right)
$$

decreases monotonically for increasing $\left|v_{\text {out }}(t-1)\right|$, this condition is satisfied if:

$$
u\left(T_{d}\right)<T_{d}
$$

and

$$
u^{\prime}\left(T_{d}\right)=-\gamma_{1} \cdot \gamma_{2} \cdot e^{-\gamma_{1} \cdot T_{d}}<1
$$

It is advised always to check Eq. (6.A7) after $\gamma_{1}, u\left(T_{d}\right)$ and $u(0.5)$ have been chosen. 


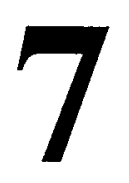

Application of Level-Dependent Spatial Summation to Clinical Data 


\subsection{Introduction}

\subsubsection{Level-Dependent spatial Summation}

A new ultrasound image processing technique has been introduced in Chapter 4. In this method, Level-Dependent spatial Summation (LDS) is applied to the twodimensional amplitude distribution comprising a B-mode image. LDS has been adapted from Intensity-Dependent spatial Summation, which was introduced by Cornsweet and Yellot Jr. (1985) as a model for retina cell interaction. Essentially, around each location $(x, y)$ in the spatial amplitude distribution a block-shaped spread function is centered, with a height that is modulated by the local amplitude value $A(x, y)$ while the product height times base area remains 1 . The analysis has been restricted to the simple case that the height of the spread function equals $c \cdot A^{q}(x, y)$, where $q$ is a positive exponent and $c$ is a positive scaling constant, so that the rectangular base area $O(x, y)$ equals:

$$
O(x, y)=\frac{1}{c \cdot A^{q}(x, y)}
$$

Whereas the base area is modulated by the local amplitude level, the ratio $k=$ $d_{x}(x, y) / d_{y}(x, y)$ of the lateral and axial dimensions of the base, remains constant. It follows that bright regions in the image produce high spread functions with narrow bases while dark regions produce low spread functions with wide bases. By summing for each location in the image the values (= heights) of the spread functions that cover that location, a spatial distribution $M$ is found of Mach bands (Cornsweet and Yellot Jr., 1985). The signal level in the $M$ distribution fluctuates around 1, whereby local peaks indicate the positions of speckle granules in the original B-mode image as well as the edges of structures.

Supplementary to the Mach bands distribution, a second distribution $N$ is obtained by counting for each location $(x, y)$ in the image the number of spread functions that cover that location. The local signal level in the spatial $N$ distribution has been shown (Chapter 5) to be sensitive to the local mean amplitude level in the original image. For a positive $q$ in Eq. (7.1), high $N$ values indicate dark regions in the Bmode image while low $N$ values correspond to bright regions.

By combining the information included in the $M$ and $N$ distributions, the original B-mode image can be modified. First, multiplying speckle peaks in the B-mode image by a factor smaller than 1 reduces the average speckle size, which may lead to an improved perceptibility of subtle brightness differences (Bamber, 1993). 
Second, global edges of hypoechoic structures are recognized on the basis of peaks in the $M$ distribution coinciding with above average values in the $N$ distribution. Edge enhancement is achieved by multiplying the edge amplitude points detected by a factor larger than 1 . Third, by multiplying each point in the B-mode image by a factor that varies with the local $N$ level, contrast between different structures in the image is increased.

In Chapter 5, the $N$ distribution has been regarded as an independent process, whereby, in contrast with Chapter 4 , exponent $q$ was allowed to be positive as well as negative. It was shown that the $N$ distribution, displayed as a gray scale image, leads to an improved detectability of slightly contrasting structures, such as lesions.

\subsubsection{Signal processing parameters}

Central parameter in the process of LDS is the scaling constant $c$ of Eq. (7.1). As regards the $M$ distribution, $c$ determines the width of the Mach bands that coincide with the speckle peaks in the B-mode image. In Chapter 4 , an expression was derived for the upper and lower boundaries between which $c$ has to be located to ensure a proper calculation of the Mach bands distribution (Eq. (4.8)). It turned out that the range of valid $c$ values is determined by the average speckle size (in number of sample points) as well as the average speckle trough amplitude level. Whereas the speckle size is essentially a (known) system parameter (Eq. (3.3)), the speckle trough amplitude level can be derived from the mean amplitude level of a moderate bright region in the image. As regards the $N$ distribution, $c$ was shown (Chapter 5) to influence the local mean $N$ level, the variance in $N$ and the transient response. The absolute characteristics of the $N$ process can be predicted if the (local) mean amplitude level is known (mean $N$ level and expected transient response) as well as the average speckle size (variance in $N$ ). Thus, for both the $M$ and $N$ distributions, automatic adjustment of $c$ is possible, provided that a mean amplitude level and an average speckle size are known.

It is noted that $M$ and $N$ both essentially result from the convolution of $A$ with a level-dependent kernel. As was suggested in Chapter 4, the two convolutions can be easily combined, whereby a single $c$ is used and $M$ and $N$ are calculated simultaneously. However, performance and flexibility can be increased, at the expense of a doubling of the computational cost, by separating the calculations of $M$ and $N$, since this allows the use of different values for $c=c_{M}$ and $c=c_{N}$.

The second parameter in the process of LDS is the exponent $q$ of Eq. (7.1). For the calculation of the Mach bands distribution, a value of $q$ of 1 is appropriate, but $q$ 
between 1 and 2 also works. For the $N$ distribution, an optimum performance in lesion detection was found (Chapter 5) for $q$ in Eq. (7.1) between 1 and 2 and between -3 and $\mathbf{- 5}$.

The third LDS parameter is the ratio $k$ of the lateral and axial dimensions of the bases of the spread functions. The $M$ distribution is preferably calculated using a $k$ equal to the ratio of the lateral and axial speckle cell sizes (both in number of sample points). On the other hand, the simulation experiments of Chapter 5 revealed that an optimum $N$ distribution is obtained if $k$ is adjusted such, that the spread functions have square bases if the dimensions are expressed in millimeters instead of sample points.

For a given B-mode image, the three parameters $c, q$ and $k$ completely determine the $M$ and $N$ distributions. Once the two distributions have been calculated, they can be used to modify the original amplitude distribution comprising the Bmode image. The amount of speckle reduction is determined by a factor $p_{s}$ by which all speckle tops are multiplied. In practice, a value of $p_{s}$ between 0.5 and 0.7 works satisfactorily. In the same way, a factor $p_{c}$ determines the weight of region contrast enhancement, which is achieved by multiplying each amplitude point $A(x, y)$ by the factor $\left(1-p_{c} \cdot N(x, y) / N_{\max }\right)$, where $N_{\max }$ is the maximum value found in the $N$ distribution. Contrast enhancement is maximum for $p_{c}=1$ and minimum for $p_{c}=0$. Finally, edge enhancement is governed by two parameters: a threshold $T_{N}$ that is applied to the $N$ distribution in order to recognize the edges present, and a factor $p_{e}$ $>1$ by which the edge points detected are multiplied in the enhancement stage. $A$ value of $T_{N}$ in-between the mean and maximum $N$ levels present proved to be a good practical starting point. It is noted that the three parameters $p_{s}, p_{c}$ and $p_{e}$ are essentially weighting factors that do not play a critical role in the processing of the $\mathrm{B}$ mode image, and their adjustment is rather subjective.

The computational cost of image enhancement by LDS has intentionally been kept low. By applying block shaped spread functions, image processing could be restricted to a limited number of elementary operations, especially if look-up tables are employed. Moreover, speckle reduction, contrast enhancement and edge enhancement all are achieved by point operations, so that real-time implementation is expected to be feasible. In that case, the parameters described by which the algorithm is adjusted (especially $c_{N}, T_{N}$ and the three weighting factors), can initially be assigned default values, which may subsequently be changed at will by the user, while he observes at the same time the effect on the monitor screen. 


\subsubsection{Aim of the experiments}

The assessment of the signal processing methods developed in Chapters 4 and 5 has so far been restricted to theory and both computer simulated data and phantom data of fairly homogeneous structures. To demonstrate that the processing of comparable "real" data can also lead to reduced speckle size, enhanced edges and increased region contrast, a number of signal recordings were made in clinic. In sections 7.3 to 7.5 , LDS is subsequently applied to a gallbladder image, an image of a breast tumor and a liver image.

\subsection{Experimental setup}

\subsubsection{Equipment}

Raw rf signals could be collected in real time by connecting a digital Ultramark $9 \mathrm{HDI}$ ultrasound system to a $486 \mathrm{DX} 4$ personal computer. The digital interface and the communication software required were developed in cooperation with ATL at the Department of Biophysics of the University of Limburg, The Netherlands. By recording the original $r f$ signals instead of the video image, all (unspecified and nonlinear) processing performed by the ultrasound system could be excluded. The ultrasound system was equipped with two transducers: a 4-7 $\mathrm{MHz}$ broad band curved array transducer, with a radius of curvature of $4 \mathrm{~cm}$ and a field of view of 60 degrees, and a $5-10 \mathrm{MHz}$ broad band linear array transducer with a width of $4 \mathrm{~cm}$. Rf signals were sampled at $20 \mathrm{MHz}$. The data frames of the curved array transducer consisted of a maximum of 128 scan lines of $2048 \mathrm{rf}$ sample points each, which corresponds to a depth range of approximately $8 \mathrm{~cm}$. The linear array transducer produced rf data frames with a maximum of 192 scan lines of 1024 sample points each, corresponding to a depth range of $4 \mathrm{~cm}$. For both transducers, the delay between emission of an ultrasound pulse and the subsequent taking of the first rf sample point could be varied, enabling the free selection of a region of interest.

\subsubsection{Data collection}

Echo data were collected from a number of voluntary patients during clinical routine investigation. Scans were made by experienced clinicians. By pushing a keyboard button, the rf data frame corresponding to the B-mode image present on the monitor screen was instantaneously stored on hard disk. All processing of data was performed off-line. 


\subsubsection{Signal processing}

The first step in the processing of an rf data frame involved the calculation of the spatial amplitude distribution using the Hilbert transform (section 2.3.1). For recordings made with the linear array transducer, postprocessing was directly applied to the amplitude distribution, while bilinear scan conversion (section 2.3.2) was applied afterwards. In the case of the curved array transducer, only speckle reduction was directly applied to the raw amplitude distribution while all other processing was applied after sector scan conversion. The bilinear scan converter was always adjusted in accordance with the proportions of the transducer selected and, for the curved array transducer, the delay adjusted between pulse emission and echo reception. As a final step in the construction of an image, dynamic range compression was applied, using a logarithmic gray level look-up table (section 2.3.3). Pixel values were retained between 0 and 255 .

\subsubsection{Quantification of the level of improvement}

An attempt was made to quantify the effect of signal processing. One or two regions in both the original amplitude file and the corresponding processed data file (prior to dynamic range compression) were selected and the values of (a selection of) the following parameters were calculated:

- The point signal-to-noise ratio, which is defined as the mean signal level $\mu$ as averaged over a region divided by the relevant standard deviation $\sigma$ (Eq. (3.2)). The less pronounced the speckle noise pattern in the processed image, the better the point signal-to-noise ratio. The point signal-to-noise ratio will be indicated by the symbol $S N R_{A}$ for amplitude data (original or processed) and by $S N R_{N}$ for $N$ data.

- The signal-to-noise ratio of the difference signal, which is defined as the ratio $\left|\mu_{1}-\mu_{2}\right| /\left(\sigma_{1}^{2}+\sigma_{2}^{2}\right)^{1 / 2}$, where the subscripts 1 and 2 indicate the region over which is averaged. The signal-to-noise ratio of the difference signal will be indicated by the symbol $S N R_{\Delta A}$ (amplitude distribution) or $S N R_{\Delta N}$ (N distribution). Evidently, $S N R_{\Delta A}$ and $S N R_{\triangle N}$ are associated with the point signal-to-noise ratio of the signal that is obtained by subtracting the signals of regions 1 and 2 . In addition, $S N R_{\Delta N}$ was found in Chapter 5 to be predictive for the extent to which sample points from the two different regions can be distinguished.

- Contrast between the two regions selected, which is defined as the ratio $C=$ $\left|\mu_{1}-\mu_{2}\right| /\left(\mu_{1}+\mu_{2}\right)(0<C<1)$ (Eq. (4.17)). 
- The average speckle size $S$, which is calculated as the area under the normalized two-dimensional autocovariance function of the spatial amplitude distribution, down to 10 percent of its maximum (Verhoeven and Thijssen, 1993). When possible, $S$ will be split up into the average speckle sizes in two perpendicular directions, each calculated as the area under the normalized one-dimensional autocovariance function in the respective direction (see also Eq. (3.3)). $S$ is a textural parameter that provides an indication, in addition to the point signal-to-noise ratio, of the extent to which a speckle noise pattern hampers the perception of local mean gray levels in the image.

- The lesion signal-to-noise ratio $S N R_{L}$, which is calculated as $S N R_{\Delta A}$ times the square root of the approximate number of independent speckle cells in the region that is considered to be the "lesion" (Eq. (4.16)). $S N R_{L}$ thus is a parameter in which first-and second-order statistics are combined (Smith et al., 1983; Thijssen et al., 1988).

\subsection{Processing B-mode data of the normal gallbladder}

Figure 7.1-a shows a B-mode image of the normal gallbladder (dark region near the center) as recorded with the curved array transducer. The top of the image is located at a depth of $2.3 \mathrm{~cm}$, the bottom at $10.2 \mathrm{~cm}$. The image fits in a rectangle of 877 pixels (width) by 544 pixels (height). The raw amplitude distribution comprising the image consists of 128 scan lines of 2048 sample points. Application of edge enhancement and contrast enhancement to the scan converted amplitude distribution yielded the processed image of figure 7.1-b. The level of improvement was quantified by comparing two separate square regions in the image with each other, before as well as after processing. Both regions covered a homogeneous area of about $1 \times 1$ $\mathrm{cm}^{2}$. One region was located in the center of the gallbladder, the other outside the bladder in the background at the upper left of the image. It is pointed out that the numerical results to be presented are based on the spatial amplitude distributions (original and processed) and not on the pixel values in the image, so that any influence of dynamic range compression is excluded.

The $M$ and $N$ distributions were calculated independently (i.e., with independent $c$ values), whereby in both cases block-shaped spread functions were used with square base areas $O$ inversely proportional to the local, squared amplitude:

$$
O(x, y)=\frac{1}{c \cdot A^{2}(x, y)}
$$




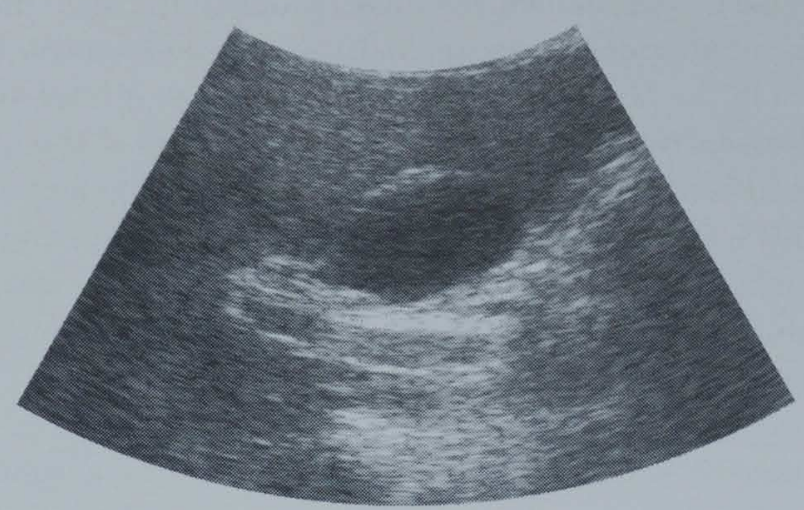

(a)

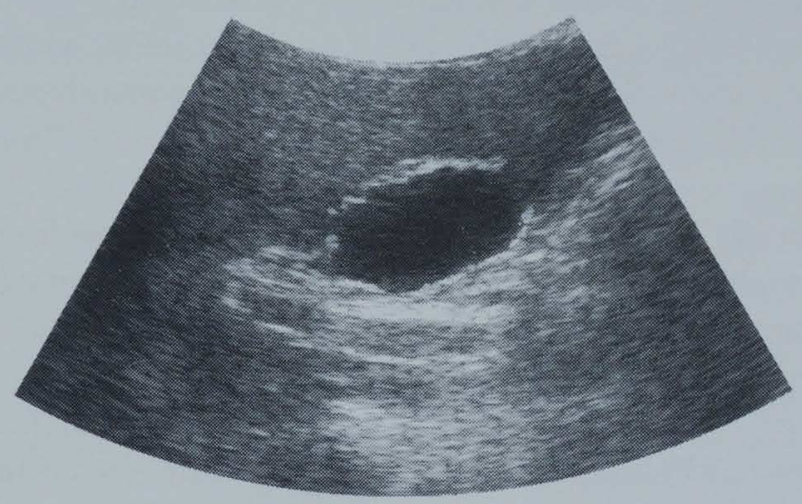

(b)

Figure 7.1. (a) Image of a normal gallbladder (dark region near the center). (b) Image after application of edge enhancement and region contrast enhancement.

In the calculation of the $M$ distribution, a rather small value of the scaling parameter $c$ was chosen: $C_{M}=0.001$ at a mean gallbladder amplitude level of 0.5 arbitrary units and an average gallbladder speckle size (measured as the area under the normalized $2 \mathrm{D}$ autocovariance function of the spatial amplitude distribution) of 27 points. The effect of a small $c$ in combination with the relation between $O$ and $A$ as defined in Eq. (7.2) is, that no peaks in the $M$ distribution occur inside the gallbladder, despite the fact that some nonzero speckle pattern is still present. As a result, the detection of false edges inside the bladder is avoided. The calculation of the $N$ distribution was also based on Eq. (7.2), but with $c$ set to $c_{N}=0.007$. This way, a mean gallbladder $N$ level of 1399 units was achieved, while the background $N$ level remained at 79 units. 
Edge enhancement was applied within a rectangular region enclosing the gallbladder, contrast enhancement was applied to the whole image. Locations in the image where peaks in the $M$ distribution coincided with an $N$ level of at least $T_{N}=1.1$ times the overall mean $N$ level, were recognized as edges of the gallbladder. Edge enhancement was achieved by multiplying the corresponding amplitude points by a factor $p_{e}=3$. Contrast enhancement was achieved by multiplying each amplitude point, except the edge points, by a factor $\left(1-p_{c} \cdot N(x, y) / N_{\max }\right)$, where $N_{\max }$ equaled the maximum value found in the $N$ distribution. The weighting factor $p_{c}$ of region contrast enhancement was set to 0.8 .

Table 7.1 summarizes the differences between the background region and the gallbladder region for the original image (left part) as well as the processed image (center part). Apparently, contrast enhancement has led to a decrease of the mean gallbladder amplitude level from 0.46 to 0.16 arbitrary units, while the background level only slightly dropped from 2.32 to 2.23 units. Consequently, mutual contrast $C$ $=\left|\bar{A}_{\text {backgr }}-\bar{A}_{\text {blad }}\right| /\left(\bar{A}_{\text {backgr }}+\bar{A}_{\text {blad }}\right)(0 \leq C \leq 1)$ increased from 0.67 in the original image to 0.87 in the processed image. The point signal-to-noise ratio $S N R_{A}=\bar{A} / \sigma_{A}$ hardly changed in the background and showed some decrease from 2.20 to 1.84 in the gallbladder (where the $N$ level reached a maximum and contrast enhancement thus had the strongest influence). However, the increase of mutual contrast still led to a net increase of the signal-to-noise ratio of the difference signal $S N R_{\triangle A}=$ $\left|\bar{A}_{\text {backgr }}-\bar{A}_{\text {blad }}\right| /\left(\sigma_{A, \text { backgr }}^{2}+\sigma_{A, \text { blad }}^{2}\right)^{1 / 2}$. The data in table 7.1 further show that contrast enhancement hardly influenced the average speckle sizes $S$ in the background and the bladder, so that the lesion signal-to-noise ratio $S N R_{L}$ increased by 15 percents.

Table 7.1. First-and second-order statistics calculated over two regions, "background" and "gallbladder", in the original gallbladder image of figure 7.1 (left), the processed image of figure 7.1 (middle) and the processed image obtained using a doubled $c_{N}$ of 0.014 (right).

Measurements were made prior to dynamic range compression.

\begin{tabular}{|c|c|c||c|c||c|c|}
\hline & \multicolumn{2}{|c||}{ original } & \multicolumn{2}{|||}{ processed, with $\mathrm{c}_{\mathrm{N}}=0.007$} & \multicolumn{2}{|c|}{ processed, with $\mathrm{c}_{\mathrm{N}}=0.014$} \\
\cline { 2 - 7 } & background & gallbladder & background & gallbladder & background & gallbladder \\
\hline$\overline{\mathrm{A}}$ & 2.32 & 0.46 & 2.23 & 0.16 & 2.26 & 0.20 \\
$\mathrm{SNR}_{\mathrm{A}}$ & 2.15 & 2.20 & 2.13 & 1.84 & 2.13 & 1.94 \\
$\mathrm{~S}$ & 25.0 & 26.6 & 25.1 & 27.5 & 25.1 & 27.0 \\
\hline $\mathrm{C}$ & \multicolumn{2}{|c||}{0.67} & \multicolumn{2}{|c|}{0.87} & \multicolumn{2}{c|}{0.83} \\
$\mathrm{SNR}_{\triangle \mathrm{A}}$ & \multicolumn{2}{|c|}{1.69} & \multicolumn{2}{|c|}{1.97} & \multicolumn{2}{c|}{1.93} \\
$\mathrm{SNR}_{\mathrm{L}}$ & \multicolumn{2}{|c|}{$100 \%$} & \multicolumn{2}{|c|}{$115 \%$} & \multicolumn{2}{c|}{$113 \%$} \\
\hline
\end{tabular}


Contrast enhancement is governed by the $N$ distribution. As follows from Eq. (7.2) and the theory in Chapter 5, the characteristics of the $N$ distribution (especially the local mean $N$ level, the local variance in $N$ and the transient response) can be influenced by changing the scaling parameter $c_{N}$. For example, a doubling of $c=c_{N}$ in Eq. (7.2) leads to a halving of each base area $O(x, y)$ and, consequently, to a halving of both the local mean $N$ level and the local variance in $N$. In addition, edge blurring in the $N$ distribution is reduced. The right part of table 7.1 summarizes the extent to which contrast enhancement is influenced if the $N$ distribution is calculated using a doubled $c_{N}$ of 0.014 (other parameters were preserved). Obviously, the results only slightly differ from those in the center part of the table, which indicates that contrast enhancement is not very sensitive to changes in $c_{N}$. The adjustment seems therefore not too delicate in practice.

Edge enhancement is governed by both the $M$ and $N$ distributions, which in turn are controlled by the scaling parameters $c_{M}$ and $c_{N}$, respectively. Figure 7.2 shows the way in which changes of the scaling parameters influence the result of edge enhancement. Figure 7.2-a shows the gray level distribution (after dynamic range compression) along a single vertical line taken from the unprocessed B-mode image of figure 7.1. The anterior wall of the gallbladder is located at $y \approx 120$ (pixels), the posterior wall at $y \approx 240$. Figure 7.2 -b presents the corresponding line in the processed image of figure 7.1, exhibiting enhanced edges of the gallbladder and a decreased average gray level of the interior of the bladder. A doubling of $c_{N}$ to 0.014 , with preservation of other settings, yielded the gray level distribution of figure 7.2-c. A doubling of $c_{M}$ to 0.002 , again while keeping the other parameters at their original values, resulted in the line of figure 7.2-d. Inspection of figures - $b$ to $-\mathrm{d}$ shows that edge enhancement too seems not very sensitive to the precise values assigned to the scaling parameters $c_{M}$ and $c_{N}$ : changes by 100 percent did not influence the positions of the first anterior and posterior edge peaks as seen from the interior of the bladder. The main effect was a variation of the width of the edge regions detected.

It is concluded that edge enhancement and contrast enhancement have been applied successfully to a clinical gallbladder image. The findings globally agree with the content of Chapter 4 . Contrast enhancement increased the lesion signal-tonoise ratio by 15 percents. Comparison of this value with the 60 percents achieved in Chapter 4 on the basis of simulated lesion images, might suggest that the present result is inferior. However, the difference can be explained by the difference in initial contrast between the two regions compared, and the fact that $S N R_{L}$ is proportional to $C$ (Verhoeven and Thijssen, 1993). In Chapter 4, $C$ was increased from 0.18 to 0.33 , which corresponds to a reduction of the ratio $\bar{A}_{\text {lesion }} / \bar{A}_{\text {backgr }}$ by a factor 1.4 . In the present experiment, $C$ was increased from 0.67 to 0.87 , which corresponds to 


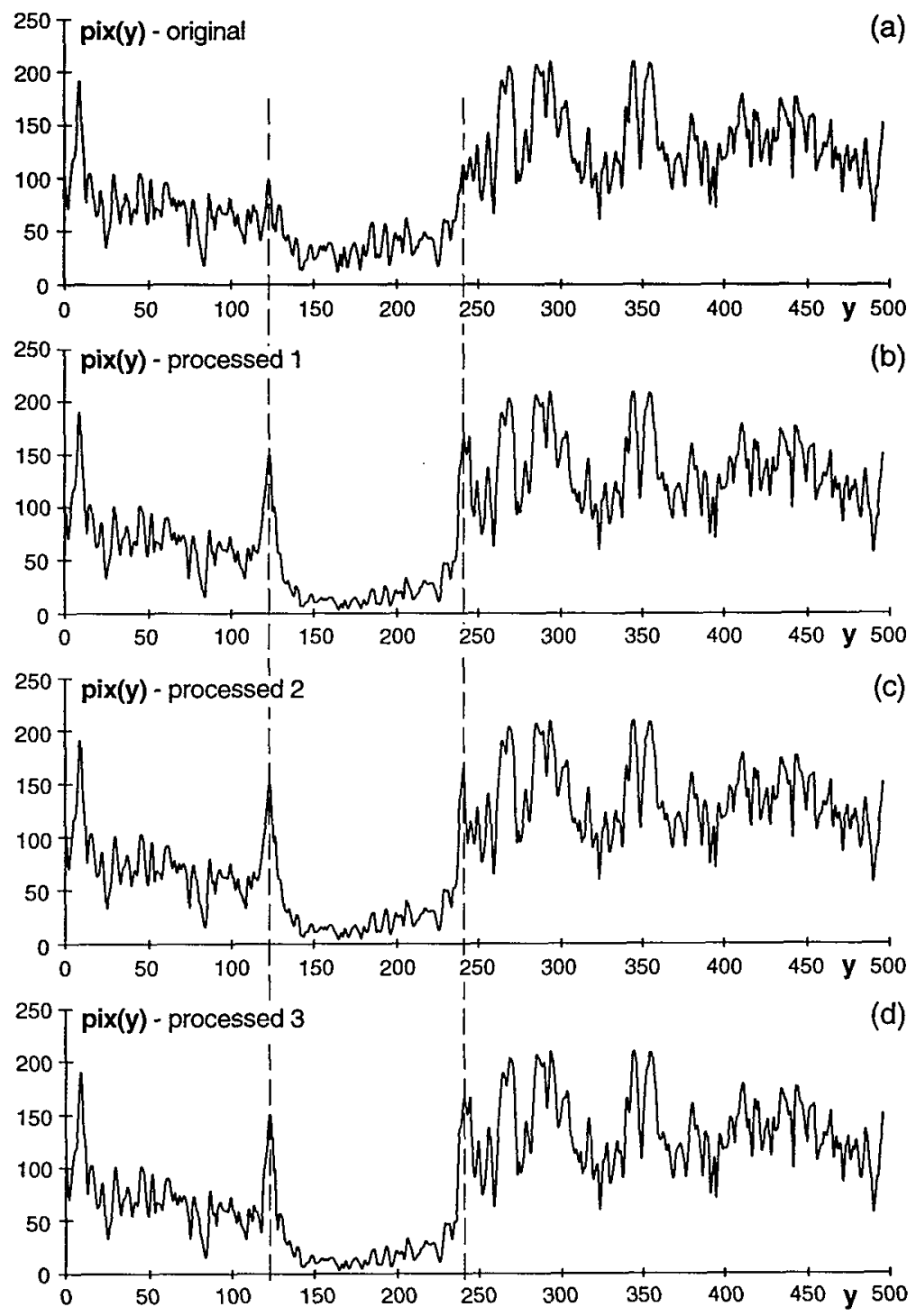

Figure 7.2. (a) Gray level distribution along a single vertical line taken from the gallbladder image of figure 7.1-a. The two dashed lines, at $y \approx 120$ and $y \approx 240$, indicate the approximate positions of the anterior and posterior gallbladder walls, respectively. (b) Corresponding gray level distribution taken from the processed image of figure 7.1-b, with enhanced edges and an increased gallbladder contrast. (c) Same as (b), except that data processing was performed using a doubled $c_{N}$ of 0.014 . (d) Same as (b), except that data processing was performed using a doubled $c_{M}$ of 0.002 . 
a reduction of the ratio $\bar{A}_{\text {blad }} / \bar{A}_{\text {backgr }}$ by a factor 2.8 , which is actually more than in the simulation experiment. Finally, it is noted that the results of Chapter 4 were based on highly homogeneous data, while the present gallbladder image is only to a certain extent locally homogeneous. A consequence of the different signal conditions is, that results may differ.

The adjustment of the scaling parameters $c_{M}$ and $c_{N}$ was found to be not too critical.

\subsection{Processing B-mode data of a breast tumor}

Figure 7.3-a shows a B-mode image of a malignant breast tumor (dark region near the center). The image was recorded with the linear array transducer described and covers an area of approximately $4 \times 4 \mathrm{~cm}^{2}$. The top is located at a depth of $0.4 \mathrm{~cm}$. The spatial amplitude distribution comprising the image consists of 192 scan lines of 1024 sample points each. The average axial and lateral speckle sizes within the tumor area equal $S_{x}=2.6$ and $S_{y}=7.8$, each measured, prior to scan conversion, as the area under the normalized $1 \mathrm{D}$ autocovariance function of the amplitude distribution in the respective direction.

The algorithm for LDS as described in Chapter 5 was applied to the raw spatial amplitude distribution. Around each amplitude point $A(x, y)$ a window was centered, covering an area $O(x, y)=1 /\left(c \cdot A^{2}(x, y)\right)$. The output $N(x, y)$ of the algorithm

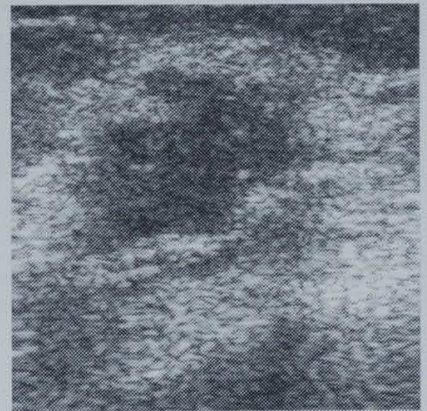

(a)

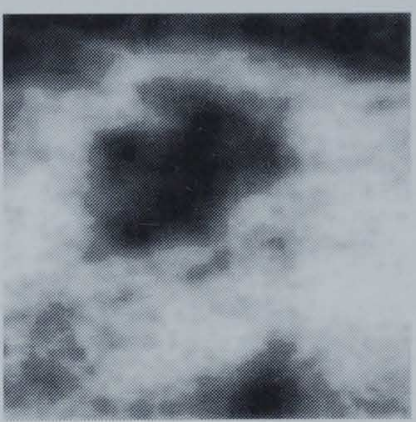

(b)

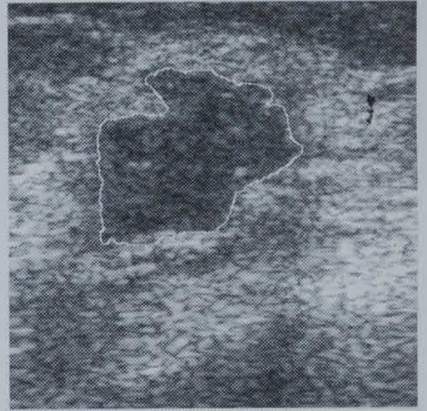

(c)

Figure 7.3. (a) Image $\left(4 \times 4 \mathrm{~cm}^{2}\right)$ of a breast tumor (dark region near the center). (b) $N$ distribution on inverted gray scale. (c) Superposition on the original image of (a) of the tumor contour automatically detected. 
included for each location $(x, y)$ the number of windows that had covered the location. This way, high $N$ values indicated dark regions in the B-mode image while low $N$ values indicated bright regions. Windows were clipped at $O_{\max }=5000$. The ratio $k$ of the lateral and axial window dimensions was set to 0.19 , which gives square windows if the dimensions are expressed in millimeters. The scaling parameter $c$ was set to 0.005 . Figure 7.3-b shows the resulting $N$ distribution on inverted gray scale.

To enable the quantification of the effect of filtering on the local first-order statistics in the image, two regions were selected in both the original amplitude distribution and the $N$ distribution: one located in the center of the lesion, the other straight below the lesion, in the moderate bright background. Each region corresponded to a fairly homogeneous area of tissue of approximately 0.6 by $0.6 \mathrm{~cm}$. Table 7.2 summarizes the first-order statistical properties as calculated over the two regions. The point signal-to-noise ratio $S N R_{A}=\bar{A} / \sigma_{A}$ approximated 1.97 in both the lesion and the background area. Application of the filter resulted in an increased point signal-to-noise ratio $S N R_{N}=\bar{N} / \sigma_{N}$ of 2.70 in the background (where the average window area $\bar{O}_{\text {backgr }} \approx \bar{N}_{\text {backgr }}=23.0$ is relatively small) and 12.5 in the lesion (where the average window area approximated the much higher level of 380). Mutual contrast $C$ equaled $\left|\bar{A}_{\text {backgr }}-\bar{A}_{\text {lesion }}\right| /\left(\bar{A}_{\text {backgr }}+\bar{A}_{\text {lesion }}\right)=0.72$ in the original amplitude distribution and $\left|\bar{N}_{\text {backgr }}-\bar{N}_{\text {lesion }}\right| /\left(\bar{N}_{\text {backgr }}+\bar{N}_{\text {lesion }}\right)=0.89$ in the $N$ distribution. The signal-to-noise ratio of the difference signal improved from $S N R_{\triangle A}$ $=1.63$ in the amplitude distribution to $S N R_{\Delta N}=11.3$ in the $N$ distribution.

The figures presented in table 7.2 are not very adequate to quantify edge preservation, although, at least, they indicate that a clear difference exists between the background and the lesion region in the $N$ distribution. Therefore, a supplementary experiment was carried out to demonstrate that edge preservation is at least sufficient to allow the automatic estimation of the tumor edges on the basis of the $N$ distribution.

Table 7.2. First-order statistics calculated over two regions, "background" and "lesion", in the tumor image of figure 7.3 (left) and the corresponding $N$ distribution (right). Measurements were made prior to dynamic range compression.

\begin{tabular}{|c|c|c||c|c|c|}
\hline & \multicolumn{2}{|c|}{ Amplitude distribution } & & \multicolumn{2}{c|}{ N distribution } \\
\cline { 2 - 3 } & background & lesion & & background & lesion \\
\hline $\bar{A}$ & 8.29 & 1.36 & $\bar{N}$ & 23.0 & 381 \\
$\mathrm{SNR}_{\mathrm{A}}$ & 1.97 & 1.98 & $\mathrm{SNR}_{\mathrm{N}}$ & 2.70 & 12.5 \\
\hline $\mathrm{C}$ & \multicolumn{2}{|c|}{0.72} & $\mathrm{C}$ & \multicolumn{2}{|c|}{0.89} \\
$\mathrm{SNR}_{\triangle \mathrm{A}}$ & \multicolumn{2}{|c|}{1.63} & $\mathrm{SNR}_{\triangle \mathrm{N}}$ & \multicolumn{2}{|c|}{11.3} \\
\hline
\end{tabular}


Analog to figure 5.7-e, the tumor may be detected by applying a threshold to the $N$ distribution. Because the clinical tumor image of figure 7.3-a has an increased complexity as compared with the simulated lesion images of Chapter 5 , the detection was restricted to a circular region roughly enclosing the tumor. All data points in the $N$ distribution exceeding a particular threshold (and which appear more or less dark in the inverted image of figure 7.3-b) were recognized as tumor points, all others within the region of interest as background points. Because of the signal averaging properties of the filter, the tumor points detected tended to cluster, so that the locations where the tumor and background points touched, made up a closed contour by which the actual (unknown) edge of the tumor was approximated. Essential part in the detection is the selection of an appropriate threshold. This may be carried out manually, whereby one tries to choose an optimum contour by visual inspection of the B-mode image on which the contour selected is superimposed (as, e.g., in figure 7.3-c). Alternatively, as is suggested by figure 7.4, the optimum threshold can be selected automatically. Figure 7.4 shows the value that was measured of the parameter $S \tilde{N} R_{\Delta N}=\left|\bar{N}_{i n}-\bar{N}_{\text {out }}\right| /\left(\bar{N}_{i n}+\bar{N}_{\text {out }}\right)^{1 / 2}$ as a function of a threshold $T_{N}$ applied to the $N$ distribution, whereby $\bar{N}_{i n}$ is the average $N$ level as calculated over the collection of tumor points detected that touched the background (the inner

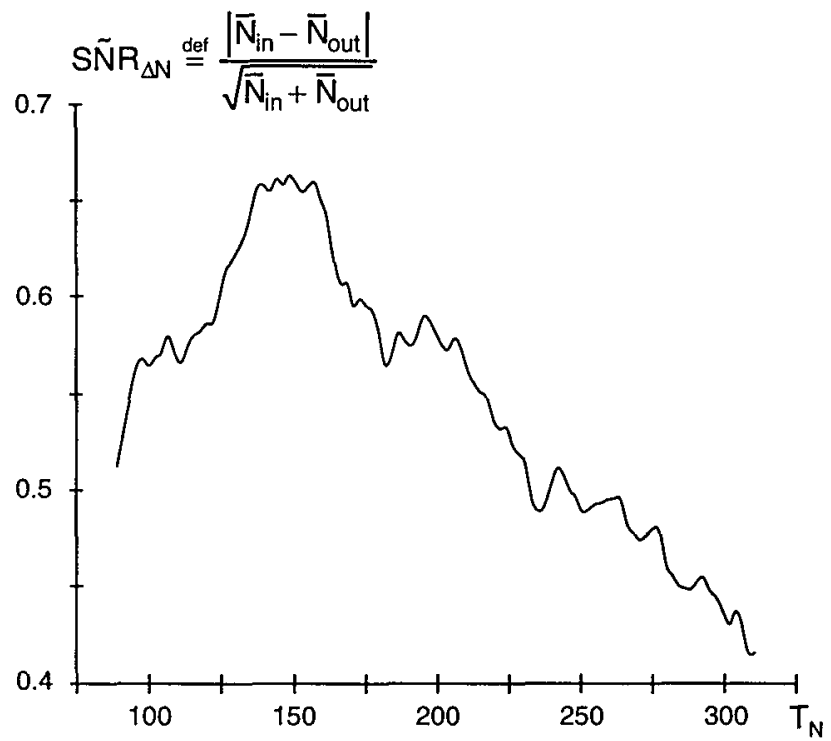

Figure 7.4. Value measured of the parameter $S \tilde{N} R_{\Delta N}$ as a function of the threshold $T_{N}$ applied to the $N$ distribution. Each particular value of $T_{N}$ yielded an inner and an outer contour of the tumor area detected, and $\bar{N}_{\text {in }}$ and $\bar{N}_{\text {out }}$ are the respective mean $N$ levels measured over the two contours. 
contour) and $\bar{N}_{\text {out }}$ is the average $N$ level as calculated over the collection of background points detected that touched the tumor (the outer contour). Evidently, for a given $T_{N}$, the outer contour always just enveloped the inner contour. As is indicated by Eq. (5.19), the local mean $N$ level tends to be proportional to the local variance in $N$. Hence, the parameter $S \tilde{N} R_{\Delta N}$ may be regarded as a measure of the signal-noiseratio of the difference signal that is associated with the two levels $\bar{N}_{\text {in }}$ and $\bar{N}_{\text {out }}$. The plot of figure 7.4 shows a marked peak around $T_{N} \approx 147$, which implies that for a limited range of thresholds the relative difference between $\bar{N}_{\text {in }}$ and $\bar{N}_{\text {out }}$ reached a maximum. Ergo, the corresponding contours might be regarded as estimates of the actual edges of the tumor. The width of the small plateau in figure 7.4 corresponds to 7 gray levels on a scale from 0 to 255 . Because the relative difference between $\bar{N}_{\text {in }}$ and $\bar{N}_{\text {out }}$ was largest around the peak, variation of the threshold within the peak region only led to marginal expansion and shrinking of the tumor area detected, so that assignment of a value to $T_{N}$ was not too delicate. Figure 7.3-c shows the contour as found when a threshold $T_{N}$ of 141 is applied to the $N$ distribution. Since the shape of the actual contour is unknown, no exact statements can be made as regards the error made in the approach, but the superposition in figure 7.3-c suggests that the contour has been "reasonably well" approached.

It is noted that the particular threshold that was applied to the $N$ distribution of figure 5.7-c, yielding the binary image of figure 5.7-e, also produced a maximum value of the relevant parameter $S \tilde{N} R_{\Delta N}$.

In conclusion, the results that have been presented in this section suggest that LDS can indeed be applied to clinical lesion images, whereby differences between lesion and background gray level distributions are enhanced (table 7.2) while lesion edges are sufficiently preserved. The results are consistent with the content of Chapter 5.

\subsection{Processing B-mode data of the normal liver}

Figure 7.5-a shows a B-mode image of the normal liver. Five cross sections of blood vessels can be discerned in the center region. The recording was made with the curved array transducer described. The top of the image is located at a depth of $4.2 \mathrm{~cm}$, the bottom at $11.3 \mathrm{~cm}$. The image fits in a rectangle of 877 by 472 pixels. The spatial amplitude distribution comprising the image consists of 128 scan lines of 1848 sample points each (sampled at $20 \mathrm{MHz}$ ). The average speckle size in the region above the 5 vessels approximates 10.2 sample points in axial direction and 2.4 sample points in lateral direction, each measured as the area under the normalized 


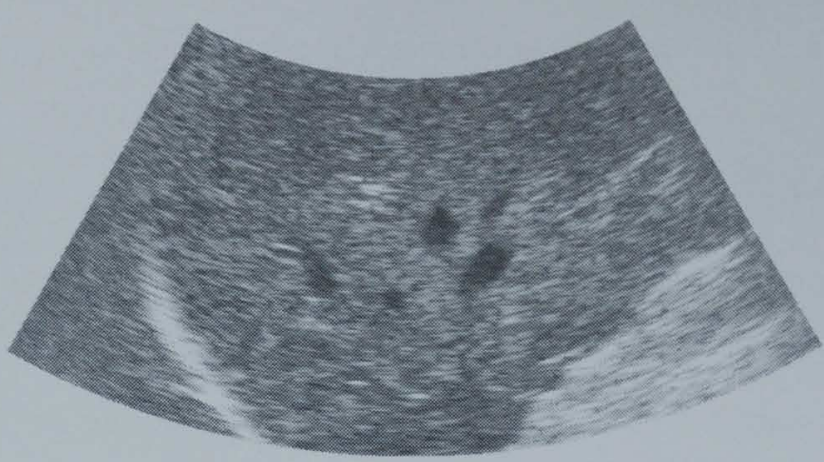

(a)

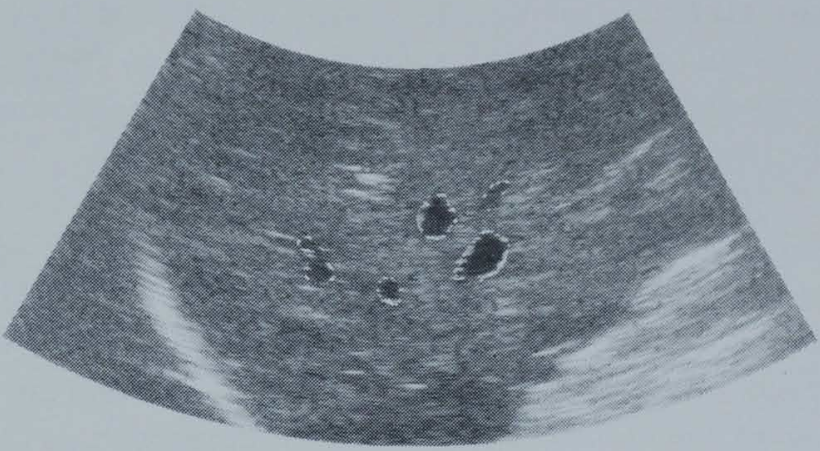

(b)

Figure 7.5. (a) Image of a normal liver. The cross sections of five vessels can be discerned.

(b) Image after application of speckle reduction, edge enhancement and region contrast enhancement.

1D autocovariance function of the amplitude distribution in the respective direction (prior to scan conversion). The mean amplitude level in the relevant region was found to equal 1.16 arbitrary units. In the same region, the point signal-to-noise ratio $S N R_{A}=\bar{A} / \sigma_{A}$ equaled 1.65. An estimate of the average speckle trough amplitude level was obtained by multiplying the mean amplitude level by $0.5: A_{\text {trough }}=0.58$ arbitrary units. This implies that, under the assumption of a Rayleigh amplitude probability density function (Eq. (3.1)), 82 percent of the amplitude points will exceed $A_{\text {trough }}$.

\section{- speckle reduction}

As described in Chapter 4, LDS can be used to reduce the average speckle size. A spatial distribution $M$ of Mach bands was obtained by applying LDS to the raw amplitude distribution of 128 by 1848 sample points. Thereby, block shaped spread 
functions were used with base areas $O$ inversely proportional to the local amplitude:

$$
O(i, j)=\frac{1}{c \cdot A(i, j)}
$$

where $i$ and $j$ denote scan line and sample point, respectively. It is noted that, although the distribution $A(i, j)$ is actually spread out over a sectorial area, $A(i, j)$ was handled as if $i$ and $j$ were rectangular coordinates. This had the advantage that the orientation of speckle cells remained constant throughout the amplitude distribution and the textural parameter speckle size $S$ could be split up in the perpendicular components $S_{i}$ (lateral) and $S_{j}$ (axial). This way, the ratio $k$ of the lateral and axial window dimensions could be set to the approximate ratio $S_{i} / S_{j}: k=0.25$. As a result, windows were square if the dimensions were expressed in number of speckle cells covered in the two directions.

As regards the scaling parameter $c$, an expression for the upper and lower bounds was derived in Chapter 4 (Eq. (4.8)) between which $c$ has to be located. It was shown that the boundaries depend on the average speckle size as well as on the average speckle trough amplitude level. Replacing $S_{x} \cdot S_{y}$ in Eq. (4.8) by the product $S_{i} \cdot S_{j}$, and substituting the values given in the first paragraph, led to the following range of valid $c$ values: $0.004<c<0.018$. From this range, the particular value 0.008 was assigned to $c$.

Speckle reduction was achieved by multiplying all speckle peak amplitude points, indicated by an $M$ value larger than 1 , by a factor $p_{s}=0.5$, while preserving the speckle trough points. This way, the average axial speckle size dropped from 10.2 to 7.3 points, and the average lateral speckle size dropped from 2.4 to 1.6 points. At the same time, the point signal-to-noise ratio showed some increase from 1.65 to 1.92. As a result, the processed image of figure 7.5-b may be perceived as locally exhibiting an increased homogeneity as compared with the original image. Moreover, in contrast with standard low pass filtering, the image has not been blurred.

It is noted that none of the speckle peaks in the lumens of the vessels produced Mach bands, which is a consequence of the locally low amplitude level. Consequently, speckle reduction did not influence the lumens.

\section{- edge enhancement}

In addition to reduced speckle, the processed image of figure 7.5 exhibits enhanced edges of the five vessels. Unlike the $M$ distribution of the former paragraph, 
the $N$ distribution, required for edge enhancement, was derived from the raw amplitude distribution based on rectangular $\mathrm{x}, \mathrm{y}$ coordinates (as obtained after scan conversion). The reason for this is, that the amount of edge blurring in the $N$ distribution could this way be kept approximately equal in all directions, by applying square windows $(k=1)$. This prevented image features to become more deformed in one direction than in others. Thus, the signal distributions to be mentioned in the remaining part of the processing description, all are based on rectangular coordinates. They have the same spatial shape as the images of figure 7.5 and they too just fit in a rectangular grid of $877 \times 472$ points.

In the calculation of the $N$ distribution, square windows were used with base areas $O$ inversely proportional to the local amplitude level: $O(x, y)=1 /(c \cdot A(x, y))$. A $c$ value of 0.03 was chosen, which led to a mean $N$ level, calculated over the whole image area, of $\bar{N}=51.5$. Edges were recognized on the basis of high $N$ values coinciding with peaks in the $M$ distribution: $N(x, y)>T_{N}=2.3 \bar{N}$ and at the same time $M(x, y)>1$. Edge enhancement was applied within a rectangular region of interest enclosing the five vessels. All edge points in the speckle reduced amplitude distribution were multiplied by a factor 10 . This is a large value, but adequate for demonstration.

\section{- contrast enhancement}

Analog to the processing of the gallbladder image, a (slight) enhancement of region contrast was achieved by multiplying each point in the speckle reduced, edge enhanced amplitude distribution by a factor that was derived from the corresponding point in the $N$ distribution. The weighting factor $p_{c}$ was set to 0.7 . As can be seen in the images of figure 7.5, contrast enhancement is most obvious for the lumens of the five vessels. The contrast between the interior of the largest vessel and a region of $1 \times 1 \mathrm{~cm}^{2}$ above the five vessels increased from $C=0.65$ in the original amplitude distribution to $C=0.72$ in the processed distribution. Apparently, contrast enhancement compensated for the effect that the speckle peaks in the lumens of the vessels had not been lowered by speckle reduction.

It is concluded that application of LDS to the liver image of figure 7.5-a has resulted in reduced speckle, enhanced edges of the vessels and enhanced region contrast. Similar effects were observed in Chapter 4 for artificial and phantom data. The application of Eq. (4.8) led to a correct value of $c_{M}$ in the calculation of the Mach bands distribution. This suggests that Eq. (4.8) has indeed practical value for the automatic adjustment of $c_{M}$. 


\subsection{Concluding remarks}

The small number of experiments described in this chapter should rather be regarded as illustrations of LDS than as actual "proofs" of its qualities. The results at least show that the processing effects claimed in Chapters 4 and 5 do not only occur in theory and in phantom and computer simulated data, but can also be observed in actual data. The effects found are in accordance with the results of Chapters 4 and 5.

A major difficulty with this kind of experiments is, that the exact content of the images is actually unknown. This especially hampered the evaluation of lesion detection in section 7.4. In addition, the homogeneous regions in the images could only be selected by means of visual inspection. Consequently, the regions had to be kept small to ensure that they did not stretch beyond the boundaries of the structures they covered in the images. Further, evaluation of edge enhancement too had to be restricted to visual inspection.

\section{REFERENCES}

Bamber J.C. (1993): Speckle reduction, in Advances in ultrasound techniques and instrumentation, Wells P.N.T. Ed., Churchill Livingstone, New York, pp. 55-67.

Cornsweet T.N. and Yellot Jr. J.I. (1985): Intensity-dependent spatial summation, J. Opt. Soc. Am. A 2, 1769-1786.

Smith S.W., Wagner R.F., Sandrik J.M. and Lopez H. (1983): Low contrast detectability and contrast/detail analysis in medical ultrasound, IEEE Trans. Sonics Ultrasonics 30, 164-173.

Thijssen J.M., Oosterveld B.J. and Wagner R.F. (1988): Gray level transforms and lesion detectability in echographic images, Ultrasonic Imaging 10, 171-195.

Verhoeven J.T.M. and Thijssen J.M. (1993): Improvement of lesion detectability by speckle reduction filtering: a quantitative study, Ultrasonic Imaging 15, 181-204. 


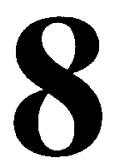

Discussion and Conclusions 
Chapter 8 


\subsection{Discussion}

In the present work, some unconventional signal postprocessing techniques to enhance medical ultrasound images have been introduced. The global philosophy behind the methods and the way they compare to existing schemes may be summarized as follows.

A major goal in the study on B-mode echo image enhancement was, to support the observer in discerning image regions of slightly different echogenicity, without masking the structural information as present in the form of edges. Thereby, speckle was regarded as the major cause of image degradation. Objectively, speckle noise limits contrast discrimination, thus decreasing the detectability of focal lesions. Subjectively, speckle distracts the observer, this way hampering the extraction of relevant diagnostic information. Speckle reduction methods described in literature were classified as signal averaging techniques, decreasing speckle gray level variance at the expense of resolution in space or time. The technique proposed in Chapter 4, in contrast, has only limited influence on relative gray level variance. Instead, it redistributes speckle noise power over an enlarged bandwidth. This way, the usual side effect of blurring of the echo image is avoided, while it was argued that the modified speckle texture may be perceived as more homogeneous, depending on image magnification and viewing distance. As a result, subtle differences in local mean gray level in the image might by easier to notice. A small extension of the method was suggested, by which additional information regarding the local mean gray level in the original image becomes available. This information was shown to enable the enlargement of global image region contrast, and to allow the enhancement of edges in all directions, two issues that seem to have barely been addressed in the context of real-time echo imaging. In Chapter 5, the novel approach to estimate local mean gray level in the presence of speckle noise was generalized. A theoretical analysis of the method in combination with experimental results revealed that the output of the estimator, displayed as a gray scale image, leads to an improved detectability of focal lesions as compared with the optimum conventional (fixed window size) local mean signal estimator in $\mathrm{B}$-mode echo imaging: the $\mathrm{L}_{2}$ mean filter. Although the study has been restricted to ultrasound images, no obstacles exist, in principle, to apply the new mean signal estimator to any other type of image. Of course, this needs further investigation.

As regards color velocity imaging, a general lack in consistency between the spatial shapes of blood velocity profiles in successive images was identified as a major source of dissatisfaction in real-time observation. The rapid and unpredictable fashion in which profiles tend to alter are likely to distract the observer. Although 
literature on this subject was found to be very sparse, this viewpoint is supported by the fact that commercial ultrasound systems are commonly equipped with some kind of temporal low pass filter (persistence filter) that provides the desired smooth transitions between consecutive profiles. However, as a side effect of low pass filtering, the true velocity information measured is affected and instantaneous peak velocities become masked. In Chapter 6, an alternative filter was developed that adapts its characteristics to the temporal blood velocity signal locally measured. It was demonstrated that this filter creates a persistence effect too, but the true velocity information measured is preserved.

Apart from supposed safety, low-budget equipment and low cost of clinical use, real-time interactivity seems to be the greatest strength of ultrasonic imaging. It enables the user to search for an optimum plane of observation in an iterative process in which manipulation of the probe immediately leads to change in target view on the monitor screen. Thereby, the user is allowed to optimize the images displayed while scanning, by freely operating system controls like receiver gain and monitor contrast. Moreover, real-time imaging allows the user to investigate structure motion as well as blood flow dynamics. Evidently, any signal postprocessing technique meant to improve image quality should be real-time implementable as well, a requirement that places a strong restriction on the complexity of processing schemes, and which limits, in principle, the level of image enhancement achievable as compared with off-line data processing. Thus, computational simplicity has been an important aim in the development of the present algorithms. Although none of the techniques has been implemented in hardware yet, the reflections made concerning the type and number of operations that have to be carried out in the processing of a frame, seem to indicate that real-time operation is indeed feasible. For all techniques developed, parameter adjustments are preferably carried out interactively, i.e., starting with some default values, the observer should be allowed to alter processing variables while simultaneously observing the effect on the output image. This seems to be the fastest and most effective procedure, at least for the experienced user, to optimize settings.

The processing schemes proposed were investigated on the basis of theoretical analyses, leading to general predictions concerning properties and behavior, and on the basis of experimental applications to phantom data and simulated data, allowing statements to be made regarding the performance under specific, controlled conditions. In Chapter 7 , three examples were presented of the processing of actual clinical B-mode images. A major difficulty encountered was, that the exact content of these images is unknown. An attempt was made to overcome this problem by restricting the performance evaluation to a number of 
small, homogeneous regions in the images that were conservatively selected by means of visual inspection. The results thus obtained indicate that, for the three particular cases, image processing was successful. Although these findings may not be generalized to all B-mode images, they are at least encouraging.

At this stage, predictions regarding the viability of the present schemes in clinical practice are hard to make. Users tend to be conservative, preferring familiar data display conventions over new alternatives. In the author's opinion, the data display format introduced in Chapter 4 is not to be expected to replace the traditional B-mode echo image entirely. The processed image should rather be viewed as a supplementary aid, which, displayed on a second screen, can be freely employed by the observer. The practical usage of the local mean signal estimator of Chapter 5 does not differ from the application of a standard moving average filter to improve the detectability of slight contrast differences in the image. Again, a second screen to display the processed image is desired, especially because of the difference in resolution between the original and the processed image. In contrast, the algorithm described in Chapter 6 to improve the presentation of color velocity images may be applied without additional provisions. Since the preservation of velocity information has been a major design goal in this study and because the persistence effect achieved is already familiar to the clinician, a secondary screen is not likely to be necessary.

\subsection{Conclusions}

The following conclusions may be drawn from the study reported:

- In real-time applications, where fast operation is essential, echo amplitude is reasonably well approached on the basis of a 6-point time domain Hilbert transformer, for narrow band as well as broad band rf signals. An alternative approach, in which pairs of adjacent rf sample points are treated as quadrature components, is only acceptably accurate (without additional provisions in the form of envelope smoothing) if narrow band $\mathrm{rf}$ signals are sampled at four times the central ultrasound emission frequency (Chapter 2).

- Subsample volume Doppler processing and rf correlation interpolation are comparable blood velocity estimation techniques as regards capacity to recognize and suppress non blood velocity estimates in color velocity imaging (Chapter 2). 
- Both spatial and temporal postprocessing of blood velocity profiles is necessary in order to achieve consistency in a sequence of color velocity images (Chapters 2 and 6).

- Level-dependent spatial summation is a new and conceptual simple image processing technique which, applied to a B-mode echo image, provides information (in the form of a distribution of Mach bands) regarding the positions of individual speckle peaks and speckle troughs, and in addition, information (included in the $N$ distribution) concerning the local mean gray level in the original image. The data obtained can be employed to reduce speckle, to enhance edges and to enhance global region contrast in the original image. Real-time implementation is expected to be feasible (Chapter 4).

- Level-dependent spatial summation can lead, via the $N$ distribution, to an improved detectability of focal lesions in B-mode images as compared with whatever conventional moving average filter (Chapter 5 ).

- By adaptive, temporal filtering of color velocity profiles in real-time color velocity imaging, a persistence effect can be created without affecting the true velocity information measured (Chapter 6). 


\section{New Concepts in Ultrasound Image Processing and their potential for improving data presentation}

\section{- summary -}

The present study deals with the processing of medical ultrasound images and aims at an improved communication of diagnostic relevant information to the clinician.

Two widely used imaging modes are of particular interest. In brightness mode or $B$-mode echo imaging, ultrasound is employed to construct a gray level monitor image that includes anatomical information of a two-dimensional section of a structure investigated, such as an organ. In color velocity imaging, ultrasound based measurement of instantaneous blood velocity distributions is added to B-mode echo imaging. This way, a gray level anatomical image is constructed in which the interiors of flowing blood containing structures are filled with two-dimensional blood velocity profiles, encoded in color. The two imaging modes both operate in real-time, which means that data collection and image construction are sufficiently fast to evoke a cinematographic effect that enables structure motion and blood flow dynamics to be visualized. (Chapter 2).

Ultrasound images can provide an indication of the presence and extent of certain abnormalities, such as, e.g., tumors and stenoses. However, the amount of information the observer gains by exploring the images is restricted. On the one hand, the characteristics of ultrasound limit the detail to which targets can be investigated. On the other hand, human vision limits the extent to which the information displayed can be absorbed. In B-mode echo imaging, speckle noise is a major source of image degradation. Speckle is a product of interference of ultrasound. It gives the images a mottled, grainy appearance. As a result, structural information is covered up, and, because of the high sensitivity of human vision to typical speckle noise patterns at normal viewing distance, the observer is likely to be distracted. Conventional image processing techniques reduce speckle by signal averaging, this way sacrificing some resolution in space or time.

In color velocity imaging, lack of spatial and temporal consistency in colored blood velocity profiles is a major cause of image degradation in real-time observation. Especially annoying is the random and unpredictable way in which the boundaries of the profiles alter from one image to the next. This effect is conventionally reduced by temporal low pass filtering of the spatial blood velocity profiles. This 
way, however, the true blood velocity information measured is influenced as well, which may lead to an erroneous reflection of flow reality, especially under dynamic flow circumstances. (Chapters 2 and 3 ).

In Chapter 4, a new approach to ultrasonic B-mode image processing is introduced. In this method, Level-Dependent spatial Summation (LDS) is applied to the two-dimensional amplitude distribution comprising a B-mode image. Essentially, the distribution is convolved with two block-shaped kernels. Both kernels have a height and a base area that are not constant, but vary with the local amplitude level. This way, two supplementary signal distributions are derived: a distribution $M$ of so-called Mach bands, indicating the positions of speckle peaks and the edges of structures in the original B-mode image, and a distribution $N$, including information about the local mean gray level in the original image. The $M$ and $N$ distributions are closely related to each other and can be calculated simultaneously. The information this way obtained regarding high spatial frequencies ( $M$ distribution) and low frequencies ( $N$ distribution) is employed to modify the original $\mathrm{B}$-mode image. First, multiplying speckle peaks in the B-mode image by a factor smaller than 1, while preserving the speckle troughs, reduces the average speckle grain size. As a result, the image may be perceived as exhibiting increased local homogeneity, while blurring of the image, usual side-effect of low pass filtering, is avoided. Second, global edges of hypoechoic structures are recognized on the basis of peaks in the $M$ distribution coinciding with above average values in the $N$ distribution. Edge enhancement is achieved by multiplying the edge points detected by a factor larger than 1 . Third, by multiplying each point in the B-mode image by a factor that varies with the local $N$ level, contrast between different structures in the image is increased. The method is evaluated in Chapter 4 using computer simulated data and phantom data. Because of the relatively low computational cost (only point operations are involved in the modification of the B-mode image), realtime implementation is expected to be feasible.

In Chapter 5, the concept of the $N$ distribution is generalized. A detailed theoretical analysis is presented as well as the results of computer simulation experiments. These indicate that the $N$ distribution, directly presented to the observer as a gray level image, is superior to the output of the optimum conventional moving average filter (the $\mathrm{L}_{2}$-mean filter) in lesion detection tasks.

Whereas the spatial $\mathrm{L}_{2}$-mean filter has limited performance as regards lesion detection in B-mode echo images, an ordinary temporal low pass filter fails in improving the presentation of real-time color velocity images: although the spatial and temporal consistency of blood velocity profiles is improved, sudden changes in blood 
flow become masked. In Chapter 6 , a technique is introduced that combines the desired improvement in consistency with the preservation of all blood velocity data available. The method is presented as a modification in two steps of a first-order recursive filter, which processes each temporal (one-dimensional) velocity signal in a spatial blood velocity profile separately from the others. The extensions make the filter adapt its characteristics to the blood velocity signal locally measured. This way, a persistence effect is created without affecting any of the true velocity information measured. As a result, the consistency in a sequence of blood velocity profiles is increased and the definition in the images of the regions that contain flowing blood is improved. The method is evaluated in Chapter 6 on the basis of computer simulated data as well as data taken from a flow phantom. Because of its simplicity, real-time implementation should be possible.

Finally, in Chapter 7, the application of level-dependent spatial summation is illustrated on the basis of three clinical ultrasound B-mode images: an image of the normal gallbladder, an image of a breast tumor and an image of the normal liver. The results support the finding of Chapters 4 and 5 that LDS is potentially a valuable new approach to ultrasound image processing. 



\title{
Nieuwe Concepten in de Ultrageluidsbeeldbewerking en hun mogelijkheid de presentatie van informatie te verbeteren
}

\author{
- samenvatting -
}

Deze studie handelt over het bewerken van medische ultrageluidsbeelden en heeft tot doel de overdracht van diagnostisch relevante informatie naar de arts te verbeteren.

Onder ultrageluid wordt in het algemeen alle geluid verstaan dat hogere frequenties (toonhoogtes) heeft dan de mens kan horen. De frequenties die gebruikt worden voor medische beeldvorming liggen zelfs aanzienlijk boven de gehoorgrens (een factor honderd of meer). Met behulp van een transducent die tegen de huid wordt geplaatst, kunnen korte pulsen ultrageluid het lichaam ingezonden worden. Door met dezelfde transducent de resulterende echo's op te vangen, verkrijgt men informatie over de structuren die zich onder de huid bevinden (bloedvaten, organen). Het gaat hierbij niet alleen om anatomische informatie, maar ook om lokale snelheden van bewegende structuren, met name van stromend bloed. Deze informatie wordt gebruikt voor het construeren van medische afbeeldingen op een beeldscherm.

Twee wijdverbreide manieren van ultrageluidsbeeldworming zijn in dit proefschrift met name van belang. In $B$-mode echobeeldvorming wordt met behulp van ultrageluid een grijswaarde monitorbeeld geconstrueerd, waarin de anatomie van een structuur langs een doorsnee te zien is (bijvoorbeeld een plakje lever). In kleurensnelheidsbeeldvorming wordt aan B-mode echobeeldvorming het meten van instantane bloedstroomsnelheidsverdelingen met ultrageluid toegevoegd. Op deze wijze wordt een grijswaarde anatomisch beeld verkregen, waarin de structuren die in werkelijkheid stromend bloed bevatten, zijn opgevuld met tweedimensionale, in kleur gecodeerde bloedstroomsnelheidsprofielen. De twee manieren van beeldvorming werken allebei in "real time", wat inhoudt dat het meetproces en de beeldconstructie voldoende snel verlopen voor het creëren van een cinematografisch effect. Hierdoor kunnen de bewegingen van structuren en de bloedstroomdynamica worden gevisualizeerd op hetzelfde moment dat ze worden gemeten (ultrageluidsbeelden worden typisch zo'n tien à twintig keer per seconde ververst, wat meestal voldoende is om de waarnemer de illusie te geven van een ononderbroken stroom van informatie in de tijd). Moderne ultrageluidsapparaten produceren digitale ultrageluidsbeelden. Digitaal wil zeggen dat elk beeld wordt gevormd door een fijn mozaiek van kleine beeldpunten (pixels) die elk één enkele grijswaarde of kleur hebben die 
gecodeerd is door middel van een getal. Deze constructie maakt het mogelijk om de beelden te bewerken met een computer.

Ultrageluidsbeelden kunnen een indicatie verschaffen over de aanwezigheid en de grootte van bepaalde afwijkingen in het lichaam, zoals bijvoorbeeld tumoren en vaatvernauwingen. De hoeveelheid informatie die de waarnemer kan vergaren door de beelden te bekijken is echter begrensd. Aan de ene kant beperken de eigenschappen van ultrageluid de precisie waarmee structuren kunnen worden onderzocht. Aan de andere kant beperkt het menselijk oog de hoeveelheid informatie die kan worden opgenomen. In B-mode echobeeldvorming is speckle-ruis een belangrijke oorzaak voor vermindering van de beeldkwaliteit. Speckle is het resultaat van onderlinge interferentie van ultrageluid gereflecteerd door dicht bij elkaar gelegen structuurovergangen. Het geeft de beelden een korrelig, gevlekt uiterlijk, met een willekeurige verdeling van kleine lichte en donkere gebiedjes (speckle-pieken en -dalen) waarachter anatomische structuren verdoezeld kunnen raken. Gezien de hoge gevoeligheid van het menselijk oog voor typische speckle-patronen op normale gezichtsafstand, is het aannemelijk dat speckle de waarnemer afleidt en de overdracht van informatie voor diagnose niet optimaal is. Conventionele beeldbewerkingstechnieken trachten speckle-patronen te onderdrukken door signalen te middelen, maar dit gaat meestal ten koste van de scherpte van het beeld.

Een belangrijk probleem in kleurensnelheidsbeeldvorming is, dat de gekleurde bloedstroomsnelheidsprofielen gaten en inkepingen vertonen (gebrek aan spatiële consistentie), die bovendien in ieder nieuw beeld een ander patroon vormen (gebrek aan temporele consistentie). Met name de willekeurige en onvoorspelbare wijze waarop de randen van de snelheidsprofielen veranderen van het ene beeld naar het volgende, vormen een bron van ergernis (zie bijvoorbeeld figuur 6.7). Conventioneel wordt dit effect verminderd door de bloedstroomsnelheidsprofielen in de tijd te filteren met behulp van een laagdoorlaatfilter. Op deze manier wordt echter de daadwerkelijk gemeten bloedsnelheidsinformatie eveneens beïnvloed, wat met name onder dynamische bloedstroomomstandigheden leidt tot een onjuiste weerspiegeling van wat zich in werkelijkheid afspeelt. (Hoofdstuk 2 en 3).

In hoofdstuk 4 wordt een nieuwe benadering tot het bewerken van B-mode ultrageluidsbeelden geïntroduceerd. Hierbij wordt Level-Dependent spatial Summation (LDS) toegepast op het tweedimensionale rooster van beeldpunten dat een Bmode beeld vormt. Het komt erop neer dat rond ieder beeldpunt een denkbeeldig blokje wordt gecentreerd. Essentieel is, dat zowel de hoogte van een blokje als zijn grondoppervlak bepaald worden door de helderheid van het beeldpunt waaromheen het blokje is geplaatst, en wel zodanig dat de inhoud van het blokje steeds 1 blijft 
(lichte beeldpunten produceren smalle hoge blokjes, donkere punten brede lage). Afhankelijk van de grootte van het grondoppervlak van een blokje, zal het enkele of meerdere roosterpunten bedekken. Door nu voor elke locatie in het beeld (ieder roosterpunt apart) de hoogtes op te tellen van alle denkbeeldige blokjes die die locatie bedekken, wordt een verdeling $M$ van zogeheten "Mach-banden" gevonden. De Machbanden blijken de posities aan te geven van zowel alle speckle-pieken in het originele $\mathrm{B}$-mode beeld als van de randen van structuren. Door daarnaast voor iedere locatie in het beeld te tellen hoeveel blokjes die locatie bedekken, wordt een verdeling $N$ gevonden, die informatie blijkt te bevatten betreffende het lokale gemiddelde helderheids- of grijswaardeniveau in het originele beeld. De $M$ - en de $N$ verdeling zijn duidelijk nauw aan elkaar verwant, zodat ze eventueel gelijktijdig kunnen worden berekend. De informatie die besloten ligt in de $M$ - en de $N$ verdeling, wordt gebruikt om het originele B-mode beeld aan te passen. In de eerste plaats wordt de gemiddelde speckle-korrelgrootte verkleind, door de speckle-pieken in het B-mode beeld te vermenigvuldigen met een factor kleiner dan 1 en de speckledalen ongemoeid te laten. Het resulterende beeld lijkt een verhoogde lokale homogeniteit ten toon te spreiden, terwijl het ontstaan van onscherpte in het beeld, een storend neveneffect van het gebruikelijke laagdoorlaatfilteren, is vermeden. In de tweede plaats worden de randen van donkere structuren in het beeld, zoals bloedvaten, herkend op basis van pieken in de $M$-verdeling die samen vallen met bovengemiddelde waarden in de $\mathrm{N}$-verdeling. De randen kunnen worden benadrukt door de corresponderende beeldpunten te vermenigvuldigen met een factor groter dan 1 . In de derde plaats kan het contrast tussen verschillende structuren in het beeld vergroot worden door ieder punt in het B-mode beeld te vermenigvuldigen met een factor die varieert met het lokale $N$-niveau. De methode wordt in hoofdstuk 4 zowel geëvalueerd op basis van met de computer gesimuleerde signalen als gebruik makende van signalen afkomstig van een weefsel-nabootsend object. Vanwege het relatief lage aantal bewerkingen (bij de modificatie van het $\mathrm{B}$-mode beeld zijn uitsluitend puntoperaties betrokken), lijkt toepassing in real time haalbaar.

In hoofdstuk 5 wordt het concept van de $N$-verdeling in algemene termen beschreven. Een gedetailleerde theoretische analyse wordt gepresenteerd evenals de resultaten van simulatie-experimenten op een computer. Deze wijzen erop dat de $\mathrm{N}$ verdeling, die rechtstreeks gepresenteerd kan worden aan de waarnemer in de vorm van een grijswaarde beeld, superieur is aan de output van het optimale conventionele lopend-gemiddelde-filter (het zogenaamde $\mathrm{L}_{2}$-gemiddelde filter) in lesiedetectieproblemen.

Daar waar het spatiële $\mathrm{L}_{2}$-gemiddelde filter een beperkte prestatie heeft wat betreft lesiedetectie in B-mode echobeelden, schiet een doorsnee temporeel laagdoor- 
laatfilter tekort als het gaat om de verbetering van real-time kleurensnelheidsbeelden: hoewel de spatiële en temporele consistentie van de bloedsnelheidsprofielen wordt verbeterd, worden plotselinge veranderingen in bloedstroming gemaskeerd. In hoofdstuk 6 wordt een techniek geïntroduceerd die de gewenste verbetering in consistentie laat samengaan met het behoud van alle bloedsnelheidssignalen die beschikbaar zijn. De methode wordt gepresenteerd als een aanpassing in twee stappen van een zogeheten eerste-orde recursief filter, welke elk temporeel (ééndimensionaal) snelheidssignaal uit een spatiëel bloedstroomsnelheidsprofiel apart bewerkt. De modificaties zorgen ervoor dat het filter zijn eigenschappen aanpast aan het lokaal gemeten bloedsnelheidssignaal. Zodoende wordt bereikt dat opeenvolgende snelheidsprofielen vloeiend in elkaar overlopen, terwijl de daadwerkelijk gemeten snelheidsinformatie toch niet wordt aangetast. Het gevolg is, dat de consistentie in een reeks bloedsnelheidsprofielen wordt verhoogd en dat de definitie in de beelden van de gebieden die stromend bloed bevatten, wordt verbeterd. Bovendien vertonen de beelden minder onrust wanneer ze in real-time worden waargenomen. De methode wordt in hoofdstuk 6 geëvalueerd op basis van met de computer gesimuleerde signalen alsmede op basis van signalen opgenomen van een testobject met een siliconen buis waarin bloedstroming wordt nagebootst. Vanwege de eenvoud van de techniek lijkt toepassing in real time mogelijk.

Tenslotte wordt in hoofdstuk 7 de toepassing van level-dependent spatial summation geillustreerd op basis van drie medische B-mode ultrageluidsbeelden: een beeld van de normale galblaas, een beeld van een borsttumor en een beeld van de normale lever. De resultaten ondersteunen de bevinding van hoofdstuk 4 en 5 dat LDS in potentie een waardevolle nieuwe benadering is tot het bewerken van ultrageluidsbeelden. 


\section{Publications}

Full papers:

- R.J. Collaris and A.P.G. Hoeks (1994): Ultrasonic B-mode image enhancement based on level-dependent spread functions, Ultrasonic Imaging 16, 205-230.

- R.J. Collaris and A.P.G. Hoeks (1994): Postprocessing of velocity distributions in real-time ultrasonic color velocity imaging, Ultrasonic Imaging 16, 249-264.

- R.J. Collaris and A.P.G. Hoeks (1995): Improved lesion detection by level-dependent spatial summation, Ultrasonic Imaging 17, 197-226.

- R.J. Collaris and A.P.G. Hoeks (1995): Improved lesion detection by level-dependent spatial summation, Acoustical Imaging 22, 257-262.

Abstracts:

- R.J. Collaris and A.P.G. Hoeks (1994): Ultrasonic B-mode image enhancement based on level-dependent spread functions, Ultrasonoor Bulletin 22, 19.

- R.J. Collaris and A.P.G. Hoeks (1994): Ultrasonic B-mode image enhancement by level-dependent spatial summation, Twenty Sixth Annual Meeting British Medical Ultrasound Society, 8.

- R.J. Collaris and A.P.G. Hoeks (1995): Improved lesion detection by level-dependent spatial summation, Book of Abstracts 22nd International Symposium on Acoustical Imaging, 240-241. 


\section{Nawoord}

Dank gaat in het bijzonder uit naar mijn promotor en initiator van het projekt, Arnold Hoeks. Ik heb hem leren kennen als iemand die zonder meer respect afdwingt met zijn gezond-verstand-benadering van de wetenschap. Daarnaast dank ik Jean Willigers en Peter Brands, voor hun technische ondersteuning. Speciale dank gaat tenslotte uit naar Hilde van Deurse, hoofdlaborante Echografie AZM, en de artsen van Radiodiagnostiek. Zonder hun hulp zou hoofdstuk 7 van dit proefschrift niet tot stand zijn gekomen.

\section{Curriculum Vitae}

\section{Roel Collaris}

9 augustus 1967: $\quad$ geboren te Heerlen

1979-1985: Atheneum-B, Bernardinuscollege te Heerlen

1985-1990: $\quad$ Elektrotechniek, Technische Universiteit Eindhoven hoofdrichting Regel- en Systeemtechniek afstudeerrichting Medische Elektrotechniek

1991-1996: $\quad$ promovendus (AIO), Rijksuniversiteit Limburg, vakgroep Biofysica 